
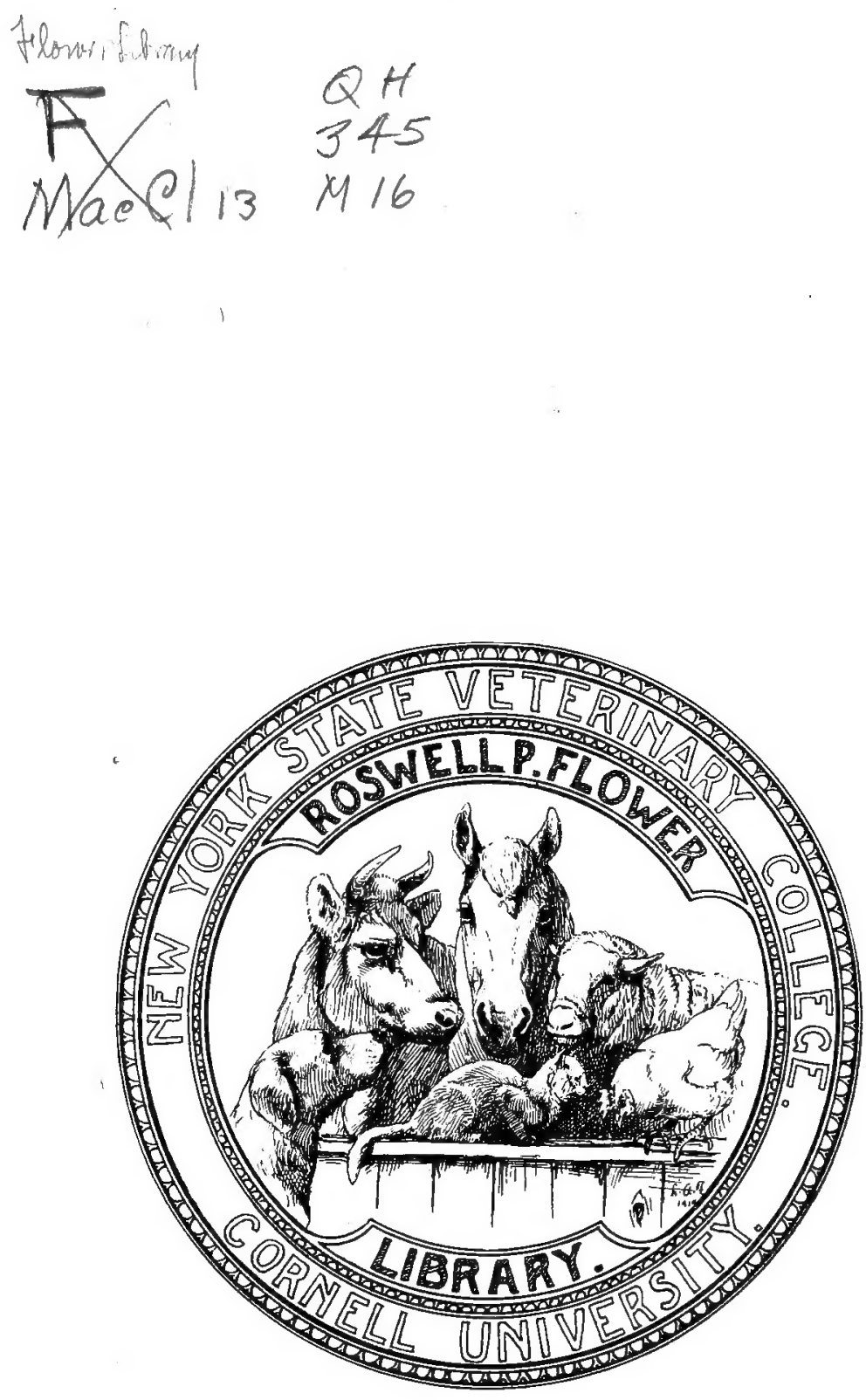
Cornell University Library

345.M16

Physical chemistry of vital phenomena, fo

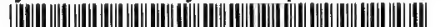

|||||||||||||||||||||||||||||||||||||||||||||||||||||||||||||||||

31924000965 842

Date Due

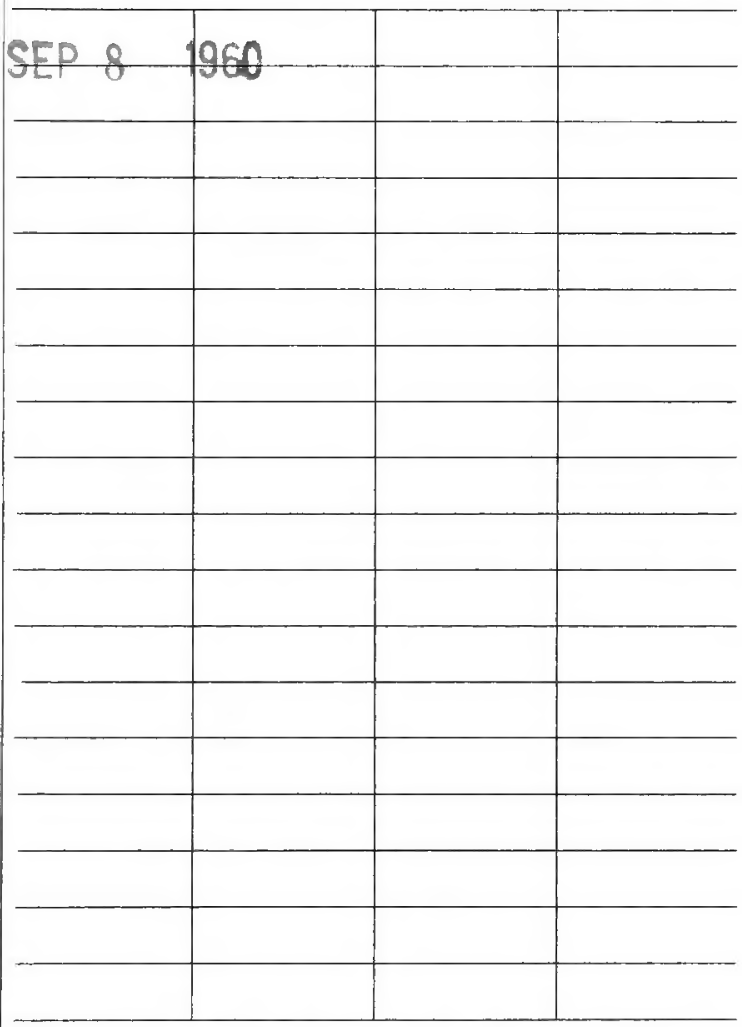

Library Bureau Cat. No. 1137 


\section{Cornell University Library}

The original of this book is in the Cornell University Library.

There are no known copyright restrictions in the United States on the use of the text.

http://www.archive.org/details/cu31924000965842 


\title{
PHYSICAL CHEMISTRY OF VITAL PHENOMENA
}

FOR

STUDENTS AND INVESTIGATORS IN THE BIOLOGICAL AND MEDICAL

SCIENCES

\author{
BY \\ J. F. MCCLENDON
}

Assistant Professor of Physiology in the University of Minnesota

PRINCETON UNIVERSITY PRESS

PRINCETON

LONDON: HUMPHREY MILFORD

OXFORD UNIVERSITY PRESS 


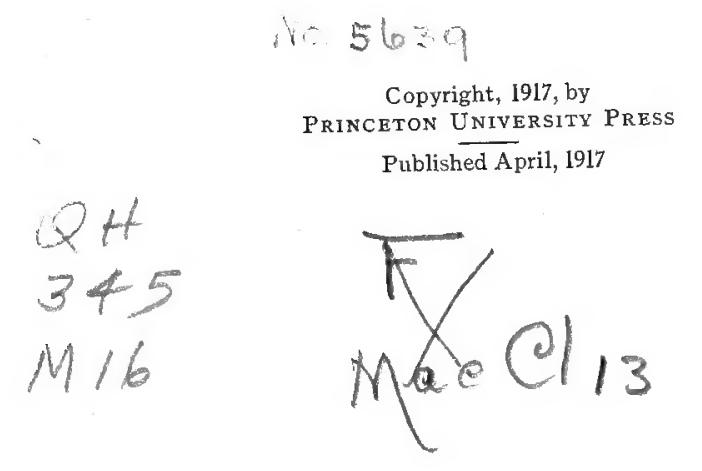


EDWIN G. CONKLIN

AND

\section{HERBERT S. JENNINGS}

WHO ENCOURAGED MY EARLY EXPERIMENTATION 


\section{PREFACE}

This book comprises a course of lectures and laboratory work given to graduate and advanced medical students in the University of Minnesota. The need for special teaching in the application of the simpler aspects of physical chemistry to biology was first realized by the author while attempting to teach physics and physiology to the same group of college students in 1906. A collection of abstracts of papers on the subject was commenced but interrupted by various affairs, especially the change to medical teaching. It became evident, however, that even medical students with a crowded curriculum would do well to devote any available time to this subject if they expect to do any research or read current medical literature.

The purpose of the book is not to go far into physical chemistry but to develop a tool for physiological research. Lengthy discussions of debated questions are avoided by tentatively accepting the hypothesis which fits the most facts, until a better one appears. For further discussion of any subject the reader is referred to the literature list and index. For facts, however, he is referred to nature. It is not to be hoped that theories should coincide exactly with data available at present. Even in the most exact branches of chemistry the atomic weight determinations, for instance, do not exactly coincide with the values calculated from the atomic numbers, and there seems to be some doubt as to whether lead is one element or several. How much more uncertainty there should be about physiology, where determinations are vitiated by the great variability of the material and its physiological states.

The literature list was compiled from abstracts made in various libraries during the last ten years in addition to published abstracts and summaries. In some cases only part of a paper or the summary was read. Following the date is the gist of some data to which it is desired to call attention, or some conclusion which the data apparently justify but which may not be ac- 
cepted by the author of the original paper. The index was made on the basis of these brief notes and hence cannot be relied on to indicate all the papers on a subject that appear in the literature list. The appendix is for the convenience of a limited class of readers.

The author expresses his sincere thanks to Professors F. E. Bartell, E. G. Conklin, I. H. Derby, G. A. Hulett, A. F. Kovarik, E. P. Lyon and others for examining the copy or parts of it and offering suggestions, and to Margaret S. McClendon for assistance in its revision.

Minneapolis, October I, Ig:16.

J. F. McClendon. 


\section{CONTENTS}

\section{CHAPTER I}

Introduction $\ldots \ldots \ldots \ldots \ldots \ldots \ldots \ldots \ldots \ldots \ldots \ldots \ldots \ldots$

Definitions of physical properties of aqueous solutions... I2 CHAPTER II

Electrolytic Dissociation

CHAPTER III

Osmotic Pressure

CHAPTER IV

Hydrogen and Hydroxyl Ion Concentration and $\mathrm{CO}_{2}$ Pres-

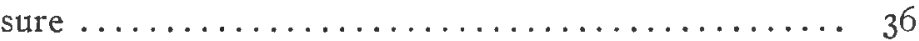

Determination of hydrogen ion concentration with hydrogen electrodes ........................ 38

Determination of hydrogen ion concentration by means

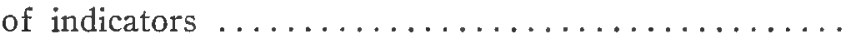

Buffers and solutions of standard hydrogen ion concentration ........................... $5 \mathrm{I}$

Dissociation constants of acids and bases......... 54

Dissociation of amphoteric electrolytes (ampholytes).... 57

CHAPTER V

Surface Tension and Adsorption............... 58

Surface tension of aqueous solutions............. 62

Adsorption $\ldots \ldots \ldots \ldots \ldots \ldots \ldots \ldots \ldots \ldots \ldots \ldots \ldots \ldots \ldots$

CHAPTER VI

Electrolytes, Non-Electrolytes and Colloids............ 68

Non-electrolytes ......................... 69

Colloids ............................ 70

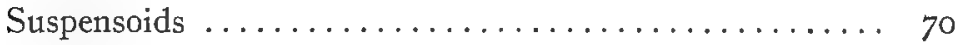

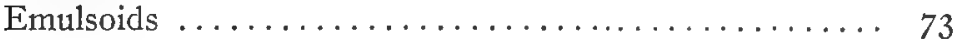

CHAPTER VII

Enzyme Action $\ldots \ldots \ldots \ldots \ldots \ldots \ldots \ldots \ldots \ldots \ldots \ldots . \ldots \ldots$

CHAPTER VIII

Permeability of Cells ..................... 88

CHAPTER IX

Changes in Permeability of Plant Cells............. I00 


\section{CIIAPTER X}

Negative Osmose and the Polarization of Membranes in Relation to the Bioelectric Phenomena, Stimulation, Absorption and Secretion................ I04 Negative osmose ....................... I05 Electric polarization of membranes and its relation to osmose ........................... I06

Comparison of the polarization of various types of membranes

Bioelectric phenomena $\ldots \ldots \ldots \ldots \ldots \ldots \ldots \ldots$ I 8

Stimulation ...................... I 28

The all-or-none law $\ldots \ldots \ldots \ldots \ldots \ldots \ldots \ldots$. 30

Absorption and secretion ................. I30

CEIAPTER XI

Anesthesia and Narcosis .................. I 33

CHAPTER XII

Cytolysis and Disinfection $\ldots \ldots \ldots \ldots \ldots \ldots \ldots \ldots$ I 42

CHAPTER XIII

Ameboid Motion and Tropisms, Cell Division, Fertilization and Parthenogenesis ..................... I48

Cell division ........................ I 56

Fertilization ........................ I57

Artificial parthenogenesis ................. I60

CIIAPTER XIV

Muscular Contraction, Oxidation and Heat and Light Production ..........................

Striated muscle $\ldots \ldots \ldots \ldots \ldots \ldots \ldots \ldots \ldots \ldots \ldots \ldots$ I6

Smooth muscle ..................... I66

Oxidation ........................... I66

Heat production $\ldots \ldots \ldots \ldots \ldots \ldots \ldots \ldots \ldots \ldots \ldots$ I 72

Light production $\ldots \ldots \ldots \ldots \ldots \ldots \ldots \ldots \ldots \ldots \ldots \ldots \ldots \ldots \ldots$

CHAPTER XV

Blood and Other Cell Media................. I75

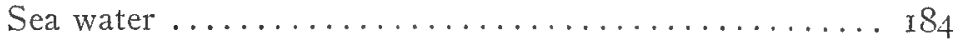

Appendix (Chemical Summary) ............ I 88

Abbreviations Used in Literature List.............. I94

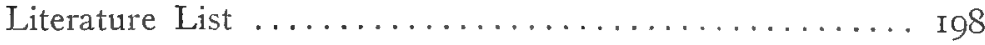

Index to Literature List. . . . . . . . . . . . . . 234 


\section{CHAPTER I}

\section{INTRODUCTION}

The chemist who turns his attention to biological problems meets at the start a seemingly insurmountable barrier. All living matter being composed of cells, and the surface of the cell in such an unstable condition that it is changed by very mild physical or chemical treatment, the rough treatment necessary for chemical analysis is out of the question. This surface layer of the protoplasm has been called by physiologists the plasma membrane. If this plasma membrane is destroyed the entire protoplasm undergoes rapid changes (cytolysis). The protoplasm, therefore, is excluded from the ordinary chemical methods of investigation, the methods that may be applied to the interior of living cells being at present very few, and concerned chiefly with the inorganic constituents.

Modern biochemistry is therefore not yet concerned directly with the composition of normal living cells, but with their decomposition products and the exchange between the cell and its surroundings. While the entire cell is treated as a unit and sometimes called protoplasm or the living substance, we have many reasons to believe that it is composed of a large number of different chemical substances, some distributed throughout the cell and others confined to certain regions, some in solution and others in the form of jellies (gels) or solids. The living cell shows a visible structure under the microscope, and chemical differences between parts of the structure may be detected after death of the cell.

From our knowledge of the diecomposition products of cells and the exchange with the medium, we may speculate on the composition of the cell and the changes that go on in it during functional activity. But before doing this the most exact quantitative data on the exchange with the environment are desirable. It is the purpose of this introduction to show that these data 
cannot be obtained or interpreted without the aid of the methods of physical chemistry. Quantitative chemistry is based on the molecular and atomic theories, and since molecules and atoms obey physical laws, physical chemistry is necessary in order to understand the reactions that take place between them. Though the problems considered in this book are physiological, the methods of attack are chiefly those of the physical chemist.

As an illustration of the usefulness of physical chemistry we may consider the grouping of the elements into the periodic system. Fig. I shows the elements grouped according to their physical characters. It may be seen that $\mathrm{Li}, \mathrm{Na}, \mathrm{K}, \mathrm{Rb}$ and $\mathrm{Cs}$ have corresponding positions in the curves. They also have similar effects physiologically. $\mathrm{Ca}, \mathrm{Sr}$ and $\mathrm{Ba}$ form another series, whereas $\mathrm{Mg}$ stands somewhat apart physiologically as well as physically, and the same is probably true of $\mathrm{Be}$ (near $\mathrm{B}$ ). $\mathrm{F}, \mathrm{Cl}$, $\mathrm{Br}$ and $\mathrm{I}$ form another series $(\mathrm{F}, \mathrm{Cl}$ and $\mathrm{Br}$ are indicated by spaces homologous with I).

It is sometimes supposed that very exact data are not necessary in biochemistry owing to the errors in taking samples and the individual variation of the organisms. We might be interested, for instance, in determining whether dextrose were burned in an animal, but consider the exact rate of oxidation to be of secondary importance. In reality, all qualitative distinctions are based on quantitative differences. When we say that coal does not burn in air at room temperature we mean that the combustion is very slow. Furthermore, the rate of a chemical reaction may determine the possibility of finally detecting the end products.

All chemical reactions are theoretically reversible. That is to say, not only does the observed reaction take place, but the opposite reaction is taking place at the same time at a slower rate. The most rapid reactions occur between electrically charged atoms or complexes called ions. Positive ions, called cations, are attracted to and combine with negatively charged ions, anions. If we mix an acid (containing $\mathrm{H}$ ions) with a base (containing $\mathrm{OH}$ ions) the $\mathrm{H}^{\prime}$ and $\mathrm{OH}^{\prime}$ combine to form $\mathrm{H}_{2} \mathrm{O}$. But the reverse reaction also takes place. Water dissociates into $\mathrm{H}$ and $\mathrm{OH}$ ions, but their number is so small that in all ordinary chemical work they are never detected. The reason for this lies in the difference in speed of the two reactions. The combination 


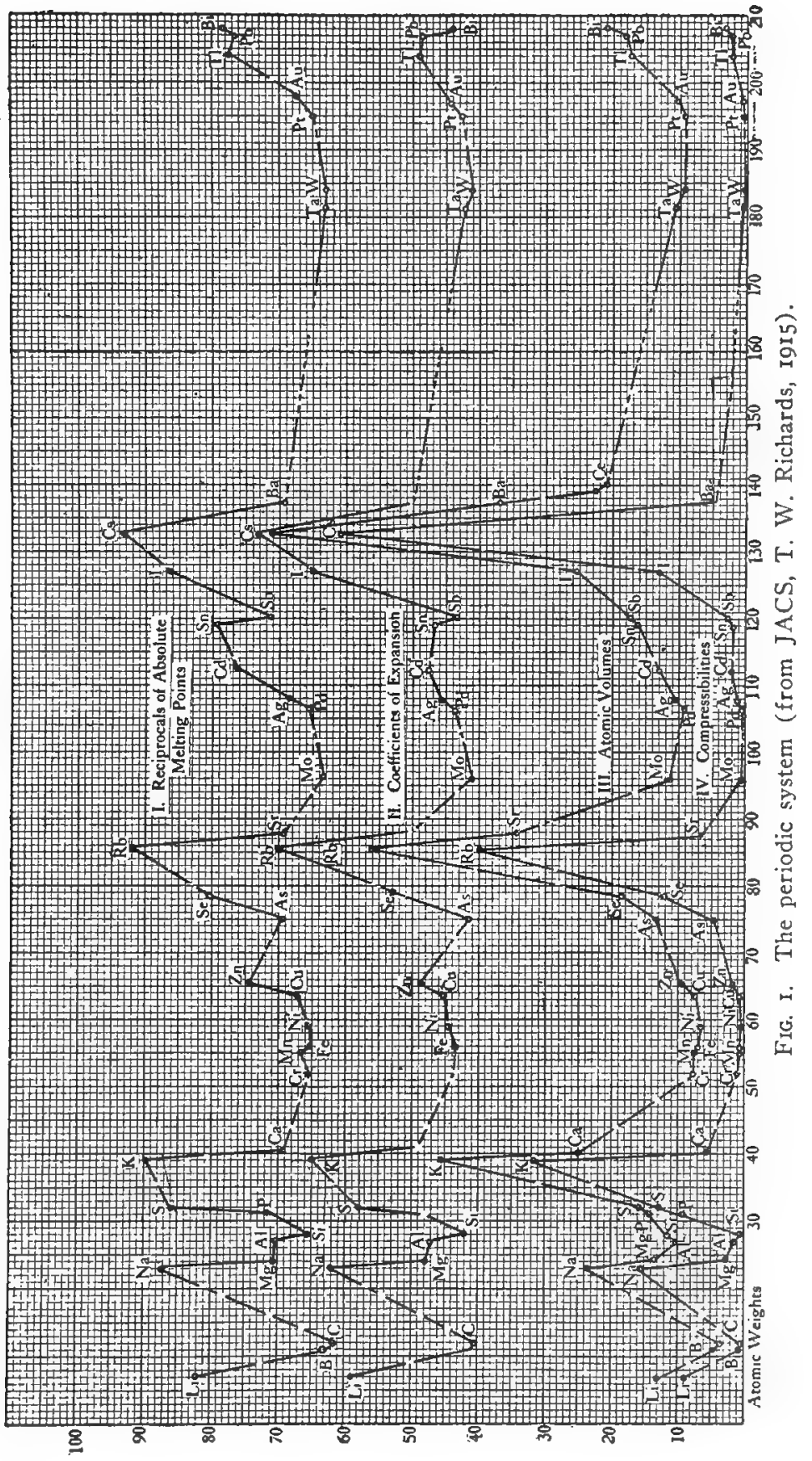


takes place with enormous rapidity and with the evolution of heat, whereas the dissociation takes place slowly. As soon as a molecule of water dissociates, its ions recombine to form water. This combination takes place whenever a hydrogen ion meets a hydroxyl ion. Hence, the speed of this reaction obeys physical laws.

Since ions of opposite charge combine when they meet, the speed of the reaction depends on the rapidity of movement of the ions and their number in unit volume, or concentration. Since the ionic speed of the same ion at constant temperature and viscosity of the solution is constant, the rate of reaction depends on the concentration. This is called the law of mass action. The behavior of the ions may be compared to men and women in a cotillion. Each man has a chance of meeting each woman. Similarly, each cation has a chance of meeting each anion, and the number of chances of a cation meeting an anion is the number of cations multiplied by the number of anions, in unit volume.

Since the concentration of the reacting substances changes during the reaction, the rate of reaction changes, and is usually negatively accelerated. The mathematical process by which the rate at any particular moment is determined is called differentiation (differential calculus). The rate is determined by the amount of substance transformed, divided by the time. If the rate were uniform we conld find it by dividing the difference in the amount of substance at the beginning and end of an observation period, by the difference in time at the beginning and end of the period. But in an accelerated reaction the rate is changing from moment to moment, and the rate at any particular time is expressed by the differential, $\frac{\mathrm{dx}}{\mathrm{dt}}$. Since the rate cannot be measured in a moment, it is necessary to use the differential calculus in order to estimate it. For our purposes, however, it is not necessary to go through all of the mathematical reasoning, but merely to use the formula supplied by the mathematician, which varies according to the conditions of the reaction.

In actual practice, ionic reactions are usually too rapid to be measured. Even the point of equilibrium may sometimes be so 
near one end as to be ascertained with difficulty. The above reactions were given as an example because their nature seerns to be better understood. The reactions whose rate can be easily measured are usually splittings and combinations of molecules. Many of these have been found to obey the laws of mass action. A monomolecular reaction in unit volume is expressed: $\mathrm{dx}$

$\frac{d x}{d t}=c(a-x)$, where $a$ is the original amount of substance, $x$ is the amount transformed in the time $t$, and $c$ is the constant of the reaction that it is desired to find. From the calculus we obtain: $c=\frac{I}{t} \log _{e} \frac{I}{a-x}$. In this equation $\log _{e}$ signifies the natural logarithm (to the base $\mathrm{e}=2.71828$ ), but this may be reduced to the common logarithm (to the base 10 ), abbreviated, $\log _{10}$ or $\log$. Since $\log _{10}=\log _{\theta} \times .4343$, the above formula becomes: $c=\frac{I}{t} .4343 \log _{10} \frac{I}{a-x}$. When several kinds of molecules are concerned the formulae become much more complicated, but in biochemistry we are less often concerned with the rate of reaction than with the point of equilibrium of a reversible reaction.

This equilibrium point in a reversible reaction is illustrated in the case of $\mathrm{H}_{2} \mathrm{O}$. The $\mathrm{H}_{2} \mathrm{O}$ dissociates into $\mathrm{H}^{\prime}$ and $\mathrm{OH}^{\prime}$ and these recombine to form $\mathrm{H}_{2} \mathrm{O}$. This is in one direction a bimolecular (bi-ionic) reaction, and in the reverse direction a monomolecular reaction. But this latter, the dissociation of $\mathrm{H}_{2} \mathrm{O}$, may be disregarded mathematically because the concentration of the $\mathrm{H}_{2} \mathrm{O}$ (at constant temperature) does not measurably change. At the equilibrium point $\left[\mathrm{H}^{*}\right] \times\left[\mathrm{OH}^{\prime}\right]=$ a constant, where [] denotes the concentration of what follows. At $22^{\circ}$, $\left[\mathrm{H}^{\bullet}\right] \times\left[\mathrm{OH}^{\prime}\right]=\mathrm{IO}^{-14}$ and therefore if we increase the concentration of $\mathrm{H}^{*}$ by adding acid the concentration of $\mathrm{OH}^{\prime}$ is decreased, and vice versa. In pure water $\left[\mathrm{H}^{*}\right]$ or $\left[\mathrm{OH}^{\prime}\right]=\mathrm{IO}^{-7}$ at $22^{\circ}$ and any variation from this is acid or alkaline. We may measure acidity or alkalinity either by estimating the concentration of the $\mathrm{H}^{\circ}$ or $\mathrm{OH}^{\prime}$, but the hydrogen ions are the ones usually estimated, as will be described in a succeeding chapter.

In any neutral dilute aqueous solution the concentration of the 
hydrogen and hydroxyl ions is nearly the same as in pure water. A nearly neutral reaction is very necessary to the life of protoplasm. The fluids that bathe the majority of body cells, and the waters in which aquatic organisms live are very nearly neutral in reaction, and are maintained in this condition by the presence of carbonates and phosphates (buffers), as will be described later.

The decomposition products of cells are proteins; carbohydrates, fats and fatlike bodies and neutral salts, as well as other substances that occur in small amount or which may arise from the decomposition of the above. The proteins are composed of amino acids. Amino acids have the general formula $\mathrm{NH}_{2} \ldots$ $\mathrm{COOH}$, in which the $\mathrm{COOH}$ group has acid and the $\mathrm{NH}_{2}$ group (after hydration) has alkaline properties, so that the amino acid may be nearly netutral. The proteins are formed by the union of the COOH group of one amino acid with the $\mathrm{NH}_{2}$ group of another with the elimination of one molecule of water. The number of amino acid molecules in one molecule of protein is very large and hence the molecular weight of proteins is enormous and is not very definitely settled in many cases. Before the proteins of the food are taken into the cells they are broken down into amino acids. Each cell builds up its characteristic proteins, irrespective of the proteins given as food.

The carbohydrates are aldehydes or ketones of polyhydric alcohols. Because of their aldehyde character, they are more easily burned in the body, and furnish most of the energy for muscular work. Some of them undergo a molecular rearrangement, giving rise to the lactone form without free aldehyde or ketone groups, but these lactone forms are changed back to the original type in digestion or metabolism. The carbohydrates are stored in insoluble form, and transported in soluble form (sugar).

The fats are esters (salts) of glycerine or other polyhydric alcohols, and fatty acids. The fats in adipose tissue are saturated and are insoluble in water. The fats of other cells are to a large extent unsaturated, and many of them contain a choline and phosphoric acid radical, which makes them capable of forming a colloidal solution with water. These phosphorized fats are perhaps more correctly called phospholipines, but together with cholesterine (a polyhydric alcohol) they have been called lipoids by many physiologists. 
The burning of I $\mathrm{g}$ monosaccharide yields 3.74 large calories, disaocharide 3.95 , animal or vegetable fat 9.5 , animal protein 5.7. All of this heat is produced by their burning in the body, except in case of protein, which produces only 4.5 calories as it is not completely burned, being eliminated as urea and uric acid and ammonia, etc.

The mineral constituents are apparently for the most part dissolved in the water contained in the cell and will be considered later in regard to their effect on the solubilities (aggregation states) of the proteins and phospholipines.

Among those constituents occurring only in traces the most important are the enzymes, thermolabile substances of unknown composition which accelerate certain chemical reactions. Some of the enzymes are poured out of the cells (as pepsin, for instance) so that they may be easily obtained, but many enzymes that are supposed to exist in all cells have been looked for in vain. Such are the enzymes that are supposed to accelerate the oxidation of the foodstuffs. It now appears that such oxidations are inseparably connected with the cell structure or to some substance that is destroyed when the cell structure is destroyed. The enzymes are greatly influenced in their activity by the hydrogen ion concentration. They may be rendered inactive by adsorption, or by anesthetics and they are influenced to some extent by neutral salts.

Most of the constituents of cells are called colloids on account of their physical properties. Colloids are divided into two classes, suspensoids and emulsoids. Suspensoids are merely solid particles electrically charged and suspended in water, only distinguished from suspensions by the small size of the particles, which cannot usually be seen with the ordinary microscope but only with the ultramicroscope with a powerful dark ground illumination. The suspensoids are precipitated by small quantities of neutral salts or other electrolytes (substances which dissociate into ions), because ions of opposite charge to the particles collide with them and neutralize their charge, the particles gravitating, no longer having the electric charges to hold them up.

Emulsoids are very different in some ways. The colloidal particles partly dissolve. The remainder absorbs water and swells, becoming viscid and the result is an ultramicroscopic emulsion 
of this viscous fluid. When two substances or fluids do not mix homogeneously they are called phases. In the suspensoid solution one phase is the solid particles and the other the water. In the emulsoid solution both phases contain water and colloid, but one is very rich in water and the other is relatively poor in water. Emulsoids may be precipitated with large quantities of salts in the same way that alcohol may be salted out of water, but they are not affected by the small amount of salt necessary to precipitate suspensoids. The particles of typical emulsoids cannot always be seen with the ultramicroscope, but the particles of lecithin, a phospholipine, for instance, are usually distinct. Many protein solutions which are typical emulsoids are transformed into surspensoids by boiling. This change, which is called denaturation, is caused by many substances, which are therefore used in tests for proteins. Emulsoids increase the viscosity and decrease the surface tension of water, whereas suspensoids do not. Since all substances that reduce surface tension become more concentrated in the surface film, the same is true of the emulsoids. The emulsoid in the surface film gradually changes into an elastic membrane, a so-called haptogen membrane.

Colloids have the peculiar property of forming jellies, called gels to distinguish them from their solutions called sols. The gel formed by a suspensoid consists of a spongelike structure formed of rows of colloidal particles. No structure can be seen in the gels of some emulsoids, but there is evidence for such a structure in their elasticity. An emulsoid increases the viscosity of water. The greater the concentration of the emulsoid the greater the viscosity, until very concentrated solutions have the viscosity of jelly, and are therefore gels. The viscosity of emulsoid sols and gels is not entirely dependent on concentration but is influenced by temperature and the presence of dissolved substances.

Whereas we may learn a great deal about the exchange between the cells and the exterior by studying the intake and output of the lungs, the products of digestion that are absorbed by the alimentary canal and the output of the kidneys, many cells are so far removed from these organs that much is left to be learned about their exchange. These cells are bathed by tissue juice, which is probably somewhat similar to the blood plasma. 
In fact, the blood plasma itself comes in contact with some of the cells.

The blood plasma contains amino acids, sugar and fats for the nourishment of the cells. The neutral salts in the plasma maintain the proper viscosity of the emulsoids, especially those of the plasma membranes of the cells. The carbonates, phosphates and to a small extent the proteins maintain the nearly neutral reaction of the plasma. The chief function of the serum albumin and globulin is not known; these proteins increase the viscosity of the blood and throw more work on the heart in maintaining the circulation. They are probably not absorbed by the cells because they appear to be different from the cell proteins. They probably exert a protective action on the plasma membranes of the cells and increase the solubility of certain substances, such as uric acid, by holding them in colloidal solution. Many intermediate products of metabolism and waste products are carried from certain cells to certain others by the blood. The presence of hormones in the blood, which stimulate certain organs to activity, seems to be established, but the chemistry of these substances, with the exception of adrenalin, is unknown. The plasma also contains the substances that form the fibrin of blood clots, and many enzymes whose function is not very clear.

The exchange between the cells and the fluid bathing them is caused by diffusion of dissolved substances, but if this were unlimited the dissolved substances of all of the cells would be the same, which is not the casie. Diffusion is fairly rapid through water but decreases as the viscosity increases. The increase of viscosity due to emulsoids does not appear to explain entirely the limitation of the diffusion of some substances, and the problem has never quite been solved, though many models showing how it might occur have been borrowed from pure chemistry. Traube's membranes form one series of models. If a solution of potassium ferrocyanide is brought in contact with a solution of a copper salt, a film of copper ferrocyanide is formed at the plane of contact of the two liquids. This film is easily permeable to water but impermeable to potassium ferrocyanide, copper salts and many other substances, such as sugar. The permeability of this membrane may be increased by treating it with certain solutions without destroying its mechranical continuity. During 
this process the membrane seems to change from a colloid structure to a mass of microscopic crystals.

We do not know the nature of the plasma membrane, but the normal icell is impermeable, or very poorly permeable, to certain salts and some other substances. Some fish eggs are impermeable to salts and to water. The permeability of cells is increased on stimulation, and goes back to normal during rest. All cells are permeable to a host of stubstances that reduce the surface tension of water to a marked degree, and to many substances that dissolve in certain non-aqueous media, although some substances that do not reduce the surface tension of water or dissolve in these media penetrate cells.

If any solution is separated from pure water or a less concentrated solution by a membrane to which the dissolved substance (solute) is impermeable, the solute exerts pressure on the membrane, and the water passes through the membrane into the solution. This pressure is called osmotic piressure, and depends not on the percentage of solute, but on the number of molecules and ions per unit volume. The standard concentration is the molecular weight of a substance in grams dissolved in a liter of water. This exerts an osmotic pressure of 22.4 atmospheres and is called a mol. If the molecules partly dissociate into ions the osmotic pressure is increased. The osmotic pressure is difficult to measure directly because it is difficult to obtain membranes that can stand the pressure. Since it has been found that the osmotic pressure is proportional to the lowering of the freezing point of pure water due to adding the dissolved substance, the freezing point is usually determined and the osmotic pressure calculated. When the osmotic pressure is greater on the inside than on the outside of a cell, the cell expands unless the cell wall is strong enough to stand the full pressure without stretching. In green plants the osmotic pressure is greater on the inside than in the water bathing the roots, because the dissolved substances are mostly manufactured on the inside. Plants use osmotic pressure as the force for growth, and can force asunder great rocks when they grow in a crack between them. The osmotic pressure of the blood of mammals is almost as constant as the body temperature, but we do not thoroughly understand its role in physiology. When an organ works, lange molecules are broken down into 
small ones, the number of molecules and the osmotic pressure are increased and water is drawn out of the blood. But when the organ rests, or grows, and large molectules are formed of small ones, the reverse process does not occur. At least the water does not flow in the opposite direction. The water that was drawn out of the blood in the first instance finally passes into the lymph vessels and then back into the blood, causing an increased flow of lymph, but the lymph flow never changes in direction.

The restricted permeability (called semipermeability) of the plasma membrane of muscle and nerve cells gives the clue for understanding the electric phenomena of these tissues. The resting plasma membrane seems to be permeable to some cations but impermeable to all anions. The cations of some substances that are more concentrated within the cell than outside diffuse through the plasma membrane, but they cannot go far, being held back by the attraction of the opposite electric charge of the anions. These cations on the outer surface of the plasma membrane give the whole cell surface a positive charge. If the plasma membrane is stimulated at one point, this altered region becomes permeable to anions and therefore negative in comparison with the unaltered portion. If the altered and unaltered portions are connected by means of a wire, a current flows through the wire from the unaltered to the altered region. This electric current is called the action current or the icurrent of injury, according to whether it is caused by stimulation or injury of the plasma membrane. Such bioelectric currents are very strong in the electric organs of certain fish, are also noticeable in the sensitive plants and in glands, and perhaps occur generally.

Since the body is made up of numerous cells and each cell is composed of several phases, the surface phenomena are very important. While the interiors of solutions are homogeneous, the surface film has peculiar properties, due to the strained relations of the molecules. Dissolved substanices (solutes) that reduce the surface tension of the solvent tend to become more concentrated in the surface film, whereas solutes that increase the surface tension are less concentrated in the surface film. If a solute that decreases the surface tension of one phase, becoming more concentrated in its surface film, is soluble in the second phase, it diffuses into the second phase (is absorbed by it); but 
if it is insoluble in the second phase it remains concentrated in the phase boundary, and is said to be adsorbed by the second phase.

Electric polarization, such as was described above in regard to nerve and muscle, decreases the surface tension. Hence stimulation is followed by local increase in surface tension. Surface tension changes cause ameboid movements and cell division. Some suppose that muscular contraction is due to surface tension changes, but if this is the case the surfaces concerned must be those of colloidal particles or internal structures, as the surface of the muscle fiber is not large enough to account for the force of contraction.

We thus see that a knowledge of what is ordinarily called chemistry is not sufficient for the biochemist dealing with medical or biological problems. Beside the reactions between proteins, carbohydrates, fats, and many organic and inorganic compounds as ustally considered by the biochemist, we are concerned with such physico-chemical processes as the rate of reaction and the position of equilibrium as expressed in the law of mass action, with the effect of ions, especially $\mathrm{H}^{\circ}$ and $\mathrm{OH}^{\prime}$, osmotic pressure, phase boundaries and the surface tension, diffusion, adsorption and electrical polarization phenomena that occur at the phase boundaries, with colloids and their aggregation states, whether gels or sols, with the effect of salts on these colloids, and especially on the plasma membrane which is apparently a colloidal structure, with the enzymes and their colloidal state and dissociation, and finally with certain reactions that are apparently accelerated by cell structure, presumably through the intermediation of adsorption phenomena.

\section{Definitions of Physical Properties of Aqueous Solutions}

There is a group of properties of water which are affected proportionately by the introduction of a solute. These are called by Washburn, I915, the colligative properties of the solution. If the solution is sufficiently dilute, the effect of the solute on its colligative properties is independent of the chemical nature of the solute, and determined solely by the number of dissolved particles (molecules + ions). The colligative properties are:

I-Vapor Pressure. If water is introduced into a vacuum 
it will evaporate until the pressure of the vapor reaches a certain value, dependent on the temperature. The vapor pressure of pure water at $20^{\circ}$ is $17.4 \mathrm{~mm}$, at $25^{\circ} 23.5 \mathrm{~mm}$, at $30^{\circ} 31.6 \mathrm{~mm}$, and at $37^{\circ} 46.7 \mathrm{~mm}$. The lowering of the vapor pressure by 1 mol solute at body temperature is only about $.5 \mathrm{~mm}$ and is less at room temperature since it is a relative lowering and the vapor pressure of pure water is less at lower temperature.

2-Boiling Point. The boiling point of water is raised $.54^{\circ}$ by I mol solute. (Vol. = I liter where not stated.)

3-Freezing Point. The freezing point is lowered $1.85^{\circ}$ by I mol solute.

4-Osmotic Pressure. If a mol solution is enclosed in a vessel whose walls are impermeable to the solute and permeable to the water, and immersed in pure water, water will pass into the vessel, causing a pressure, osmotic pressure, of 22.4 atmospheres.

With these colligative properties might be classed electrode potentials, since they depend on osmotic pressure. The concentration of a solute calculated from its effect on all colligative properties is the same, but may differ from its concentration calculated from other data, so the word activity is sometimes used to denote concentration calculated from colligative data.

The density of the solution is affected by the concentration of the solute, but the degree of change differs with different solutes. Hence, this property of a mixed solution cannot be interpreted unless the proportion of the different solutes remains approximately the same (as in sea water, for instance). Density is determined by weighing a certain volume of the solution measured in a pycnometer and dividing its weight by the weight of an equal volume of water. The rule has been to have the water at $4^{\circ}$ and the solution at $15^{\circ}$, but in many determinations the water is taken at the same temperature as the solution, and $25^{\circ}$ is rapidly becoming the standard temperature for all sorts of measurements.

The refractive index is also used to determine concentration. With the Abbe direct reading refractometer only a drop of the solution is necessary, and hence this instrument is used in protein analysis when the protein is pure or the accompanying solutes are known. The Zeiss interferometer gives a more accurate 
reading but requires a larger sample. One per cent of protein raises the refractive index oor per cent.

The viscosity of the solution is affected very little by neutral salts iut very much by emulsion colloids. Not only does the concentration of the emulsoid change the viscosity, but (at the same concentration) the colloidal state affects it. Apparently, the greater the hydration of the colloid the greater the viscosity. The viscosity of a solution is measured by the time required for it to flow through a capillary tube divided by the time required by pure water to flow through the tube at the same temperature.

The rotation of the plane of polarized light may be used to find the concentration of an asymmetric solute in a mixed solution since only molecules having an asymmetric atom, usually carbon, have this property. Rotation is measured with a polarimeter. Colloidal solutions may polarize light dispersed by the particles (Tyndall effect) and gels polarize light when deformed, and are then called doubly refractive or anisotropic.

The surface tension may be used as an index of the concentration of certain solutes. Neutral inorganic salts have very little effect on the surface tension, but anesthetics have a marked effect. The surface tension is found by dividing the weight of a drop of the solution by the weight of a drop of water dropped from the same pipette.

The electrical conductivity is much used to find the concentration of ions, but it depends, not only on the number, but also on the speed of the ions, and hence on the viscosity of the solution. The greater the hydration of an ion the less is its speed, hence the speed is supposed by some writers to decrease with decrease in concentration until maximal hydration occurs. When such a dilution is reached, further dilution evolves no heat, and the solution is called "dilute."

The color of a solution is determined qualitatively with the spectroscope and quantitatively with the colorimeter. A change in the quality of the color is supposed by Stieglitz to be due to change in the positions of electrons within the molecule, following an upsetting of the equilibrium by addition or removal of atomic groups. It seems probable that a change in the degree of hydration may lead to a change in color. The color of a sol may be due to the size and refractivity of the colloid particles. 
The degree of turbidity of a sol or suspension is determined with Richards's nephelometer.

In order to facilitate comparison of the results of different investigators, the standards used in measurement should always be recorded. The practice of measuring dimensions in the metric system and temperature in degrees centigrade (Celsius) is quite general, but some confusion may arise from any assumptions as to the concentration of solutions. The effect of the acceleration of gravity may be neglected in weighing, because it affects equally the weights used and the substance weighed. The buoyancy of the air is taken into consideration in the finest work and especially for very light substances. Certificates of the U. S. Bureau of Standards give weights in vacuo, hence the volume of the weights must be subtracted from the volume of the substance weighed in correcting for the buoyancy of the air.

The lengths of the two arms of the balance beam are often sufficiently different to produce an appreciable error in weighing. By determining the ratio of the lengths of the two arms a correction may be applied. Or the true weight may be found by taking the geometric mean of the weights on the two pans (after a second weighing on the opposite pan).

The standard solution or $m$, is the gram-molecular-weight of the solute in a liter of the solution, but the liter flask from the Bureau of Standards should be used at the temperature for which it was standardized. After the solution is standardized, any change in temperature changes its volume and hence burettes and pipettes must be used at the same temperature as the flask. A flask standardized at $15^{\circ}$ would hold $0.4 .0 \mathrm{co}$ to much at $30^{\circ}$, but if it is merely a question of volumetric analysis, this can easily be corrected for as the pipettes and burettes likewise hold 0.04 per cent too much. If a liter flask is standardized at $15^{\circ}$ it holds about $998 \mathrm{~g} \mathrm{H}_{2} \mathrm{O}$ at $\mathrm{I}^{\circ}, 996.3$ at $25^{\circ}$ and 995 at $30^{\circ}$ when weighed in air. This refers to the true liter (the volume of a kilogram of $\mathrm{H}_{2} \mathrm{O}$ at $4^{\circ}$ ) and not to the Mohr liter. A liter measures $1000.027 \mathrm{cc}$ but in this book $\mathrm{cc}$ is used to indicate $0.00 \mathrm{I}$ liter or milli-liter $(\mathrm{ml})$.

It is often desirable to use solutions under various conditions, and since their concentration according to the above definition may change, new definitions are substituted. Since the molecular 
weight of a substance may change upon solution, the weight in grams as given in the chemical formula ( $\mathrm{mol}$ ) is usually taken, and the solution of this in a liter is called formal but is designated here as mol in order to save space. When attention is especially directed to an element that occurs more than once in the formula, the molecular weight is divided by the number of times this substance occurs in the molecule (E gram equivalent weight), and the solution of this in I liter is then called normal. The effects of temperature are eliminated by using weight normal solutions, in which the formal or normal (gram equivalent) weight of the substance is dissolved in rooo grams of the solvent, but such solutions require a new standardization before use in burettes. The requirements of volumetric analysis are satisfied in the method used in titrating the chloride content of sea water. The results are expressed as grams of $\mathrm{Cl}$ per kilogram of sea water. The volumetric apparatus is standardized with a bottle of standard sea water at the temperature at which the titrations are to be made. It is then necessary merely to have a relative calibration of the divisions on the burette. 


\section{CHAPTER II}

\section{ELECTROLYTIC DISSOCIATION}

Electrolytic dissociation is not dependent on electrolysis as was formerly supposed. When certain substances are dissolved in water they dissociate into electrically charged ions, and these substances are therefore called electrolytes, to distinguish them from poorly dissociated, so-called non-electrolytes, such as sugar or urea. The individual ions in gases may be detected, but in solutions we are usually content with the circumstantial evidence of their existence. In biology we usually have to deal with aqueous solutions, and these are implied where others are not stated. Electrolytes that dissociate to a great extent in water dissociate much less if at all in many non-aqueous solutions.

Strong acids and bases and their salts are almost completely dissociated in very dilute solutions, the dissociation increasing with dilution. Weak acids and bases and their salts are as a rule poorly dissociated, but salts formed of a weak acid and a strong base or a weak base and a strong acid are more strongly dissociated. As a rule, inorganic acids and bases are strong and organic weak, but there are exceptions.

Solutions of all chlorides $\left(\mathrm{NaCl}, \mathrm{KCl}, \mathrm{LiCl}, \mathrm{CaCl}_{2} \ldots\right.$ contain chlorine ions, $\mathrm{NaCl}$ dissociating into positively charged $\mathrm{Na}$ ions $\left(\mathrm{Na}^{\circ}\right)$ and negatively charged $\mathrm{Cl}$ ions $\left(\mathrm{Cl}^{\prime}\right)$. These ions are never free as represented but always hydrated. Silver nitrate in solution dissociates into $\mathrm{Ag}^{\circ}$ and $\mathrm{NO}_{3}{ }^{\prime}$. If silver nitrate is added to the solution of any chloride a white cloud of $\mathrm{AgCl}$ is instantly formed because $\mathrm{Ag}^{\circ}$ unites with ' $\mathrm{Cl}$ ', the two being drawn together by their electric charges.

Hydrated cobalt ions give a pink color to their solution, but the undissociated salts (and possibly non-hydrated ions if they exist) are of different colors, as may be seen by dissolving them in solvents in which they do not ionize. Cobalt chlorid in alcohol is blue, but if water is added the solution turns pink. Cobalt 
nitrate in alcohol is purple, but on adding water it turns pink, because in the water-alcohol mixture electrolytic dissociation takes place (Noyes and Blanchard, 1900).

Electric conductivity of solutions: Solutions of electrolytes conduct the electric current and are called conductors of the second class to distinguish them from the metals which are conductors of the first class.

If an electric current is passed through a solution from two metallic conductors (electrodes) the positive ions (cations) are attracted to the negative electrode (cathode) and the negative ions (anions) are attracted to the positive electrode (anode).

The more ions there are in a solution the greater the electric conductivity, and hence the electric conductivity may be used to determine the ionization. In doing this the electrical resistance, in ohms, is measured, and the conductivity is the reciprocal of the resistance.

The electrical resistance of solutions is measured by means of the Kohlrausch method. The solution to be examined is placed in a conductivity cell which consists of a glass vessel containing two platinum electrodes. The electrodes are made of platinum foil and should stand vertically so that bubbles will not collect on them. Every time the distance between the electrodes is changed to the slightest degree the cell has to be standardized again. To avoid this, the platinum is often made thick for stiffness, or the four corners of one electrode are connected to the corners of the other by means of glass rods fused to the platinum by heat. To the upper edge of the platinum foil a stout platinum wire is welded. This is done by heating foil and wire to a white heat and welding the wire to the foil by means of a quick tap with a hammer. The wire is fused into the end of a small glass tube and a drop of mercury dropped into the tube so as to touch the platinum wire. When connecting up the apparatus, a copper wire is inserted down the tube into the mercury. The electrodes may be fastened into the cell by passing the glass tubes through a stopper that fits into the cell.

For solutions used in pure chemistry the pipette forms of conductivity cells that are on the market are very convenient. For biological fluids when only a small quantity may be obtained, the test tube form of cell shown in Fig. 2 is convenient. For tissues, the apparatus shown in Fig. 3 is useful. 


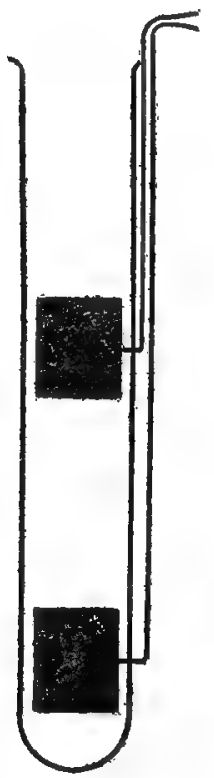

FIG. 2. Electric conductivity electrode of low inductive capacity.

Before using the cell, it must be cleaned with a saturated solution of potassium bichromate in concentrated sulphuric acid (called cleaning fluid). The platinum should be cleaned by heat before fusing in the glass, and cleaned with cleaning fluid in the cell. The cell is rinsed with distilled water and the platinum is coated with platinum black (platinized) in order to increase its

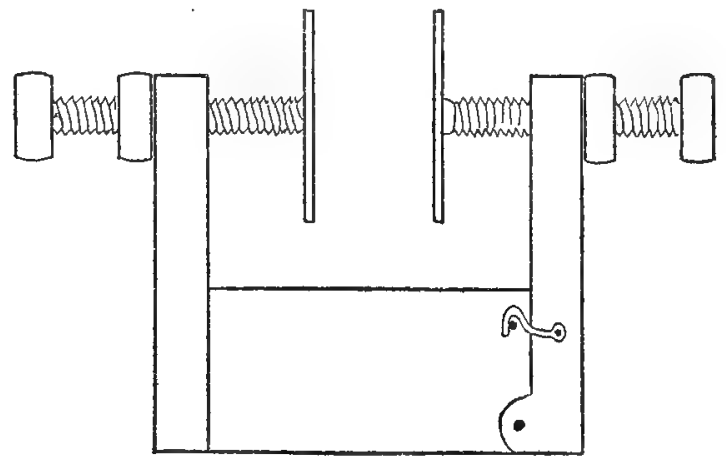

FIG. 3. Electric conductivity electrode for tissues. 
surface and reduce electric polarization. In doing this the cell is filled with about 2 per cent platinic chloride solution containing a trace of basic lead acetate. An electric current of about four volts is passed through the cell until one electrode is blackened and the current is reversed until the other pole is blackened. The platinizing solution is returned to the bottle and the cell rinsed and filled with dilute sulphuric acid. A current is now passed in both directions as before. In this way hydrogen is produced, first on one electrode and then on the other, and reduces any chlorine that may have been absorbed by the platinum. The cell is now rinsed many times, finally with conductivity water, is filled with the latter and left until used. Conductivity water is made by adding sulphuric acid and potassium bichromate to distilled water and redistilling it, then adding barium hydrate to it and distilling it the third time with the exclusion of $\mathrm{CO}_{2}$ of the air. Both distillations may be done at once (Jones, Hulett).

In order to reduce polarization still further, a rapidly alternating current is used. This is best obtained from a special electric generator, or Vreeland oscillator, but owing to its cost most people are content with a small induction coil. The vibrator of the coil should be very stiff or very short, in order to produce a high pitch resembling the sound of a mosquito. A great change in the frequency may change the resistance measured 3 per cent (W. A. Taylor, I9I5). The coil should be enclosed in a sound proof box.

A Wheatstone bridge is built up of a resistance box, the con-

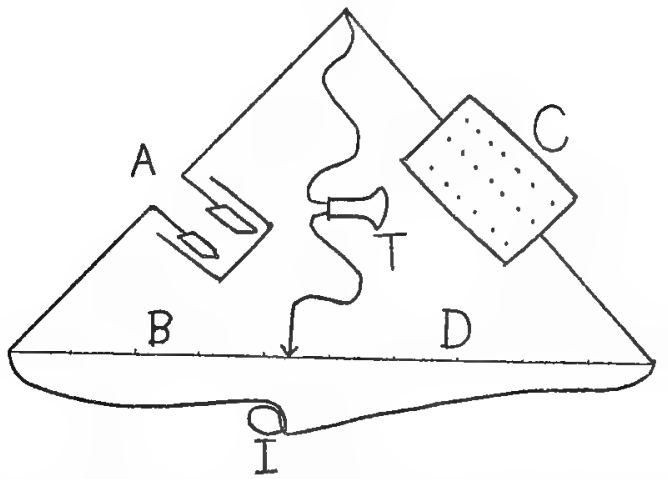

FIG. 4. Wheatstone bridge arrangement for conductivity apparatus. 
ductivity vessel, a meter resistance wire with a sliding contact, and a telephone with which to detect the current (Fig. 4). These resistances are connected in a circle. The meter wire is divided by the sliding contact into two resistances. which form two legs of the circuit, the box and the cell forming the other two legs. The two secondary poles of the induction coil are connected to the sliding contact and the juncture between coil and box respectively. The conductivity cell is marked $\mathrm{A}$ and the adjacent leg of the slide wire $B$, the box marked $C$ and the adjacent leg of the slide wire $D$. The current entering the circuit at the juncture of $A$ and $C$ divides, part going through $A$ and $B$ and the other part through $C$ and $D$. If the two ends of the meter wire have the same electrical potential, then the resistances of $\frac{\mathrm{A}}{\mathrm{B}}=\frac{\mathrm{C}}{\mathrm{D}}$, also $\frac{\mathrm{A}}{\mathrm{C}}=\frac{\mathrm{B}}{\mathrm{D}}$. In order to determine whether the two ends of the meter wire have the same potential the two wires of the telephone receiver are connected to the two ends and if no sound is heard the two ends have the same potential. If a sound is heard the resistance of the box is changed or the sliding contact on the wire is moved until no sound is heard. The resistance of the box may be read in ohms; the resistances of the two parts of the meter wire are proportional to their length, and hence their lengths are used in the equation. From the equation the resistance of the conductivity cell $A$ may be calculated. The ratio $B / D$ may be read from the accompanying table.

Usually the telephone is never quite silent, and the point of tone minimum may be more or less sharp, but may be sharpened in the following ways. The electrodes are made larger (their surface area increased). A self inductance, which may be varied, is placed in series with A. An electrical condenser, that may be varied, is placed in parallel with $C$. By readjusting the inductance and condenser and the sliding contact alternately, a sharp tone minimum is finally obtained (Washburn and Bell, I9I3). The condenser is to compensate for the capacity of the cell, which is larger the larger and closer together the electrodes are (but is least in the form of cell shown in Fig. 2). The self inductance is to compensate for the self inductance of the box and connecting wires. Resistance coils with very low self in- 
Obach's Wheatstone Bridge Table of B/D Where B = Meter Slide from o to Stiding Contact and $D=$ Remainder of Slide Wire.

\begin{tabular}{c|c|c|c|c|c|c|c|c|c|c}
\hline $\mathrm{B}$ & 400 & 410 & 420 & 430 & 440 & 450 & 460 & 470 & 480 & 490 \\
\hline 0 & .6667 & .6949 & .7241 & .7544 & .7857 & .8182 & .8519 & .8868 & .9231 & .9608 \\
$\mathrm{I}$ & .6694 & .6978 & .7271 & .7575 & .7889 & .8215 & .8553 & .8904 & .9268 & .9646 \\
2 & .6722 & .7007 & .7301 & .7606 & .7921 & .8248 & .8587 & .8939 & .9305 & .9685 \\
3 & .6750 & .7036 & .7331 & .7637 & .7952 & .8282 & .8622 & .8975 & .9342 & .9724 \\
4 & .6779 & .7065 & .7361 & .7668 & .7996 & .8315 & .8657 & .9011 & .9380 & .9763 \\
5 & .6807 & .7094 & .7391 & .7699 & .8018 & .8314 & .8692 & .9048 & .9417 & .9812 \\
6 & .6835 & .7123 & .7422 & .7731 & .8051 & .8382 & .8727 & .9084 & .9455 & .9841 \\
7 & .6863 & .7153 & .7452 & .7762 & .8083 & .8416 & .8762 & .9120 & .9493 & .9881 \\
8 & .6892 & .7182 & .7483 & .7794 & .8116 & .8450 & .8797 & .9157 & .9531 & .9920 \\
9 & .6921 & .7212 & .7513 & .7825 & .8149 & .8484 & .8832 & .9194 & .9569 & .9966 \\
\hline
\end{tabular}

In the table following, $I$ is understood before the decimal point.

\begin{tabular}{|c|c|c|c|c|c|c|c|c|c|c|}
\hline B & 500 & 510 & 520 & $53^{\circ}$ & 540 & $55^{\circ}$ & 560 & 570 & 580 & 590 \\
\hline o & .0000 & .0408 & .0833 & .1277 & .I739 & .2222 & .2727 & .3256 & $.38 \mathrm{IO}$ & .4390 \\
\hline I & .0040 & .0450 & .0877 & . 1322 & .1786 & .2272 & .2779 & .3310 & .3866 & .4450 \\
\hline 2 & .0080 & .0499 & .0921 & .1368 & .1834 & .2321 & .2831 & .3364 & .3923 & .4510 \\
\hline 3 & $.012 I$ & .0534 & .0964 & .1413 & . I 882 & .2371 & .2883 & .3319 & $.398 \mathrm{I}$ & .4570 \\
\hline 4 & .0161 & .0576 & .1008 & .1459 & . I930 & .2422 & .2936 & .3474 & .4038 & .4631 \\
\hline 5 & .0202 & .0619 & .1053 & .1505 & .1978 & .2472 & .2989 & .3529 & .4096 & $.469 \mathrm{I}$ \\
\hline 6 & .0243 & $.066 \mathrm{r}$ & .1097 & $.155^{2}$ & .2026 & .2523 & $.304 \mathrm{I}$ & .3585 & .4155 & .4752 \\
\hline 7 & .0284 & .0704 & .1142 & .1598 & .2075 & .2573 & .3095 & .3641 & .4213 & $.48 \mathrm{I} 4$ \\
\hline 8 & .0325 & .0747 & I I 86 & .1645 & .2124 & .2624 & .3148 & .3697 & .4272 & .4876 \\
\hline 9 & .0367 & .0790 & . I 231 & .1692 & .2173 & .2676 & .3202 & .3753 & .4331 & $.493^{8}$ \\
\hline
\end{tabular}

ductance may be made by bifilar winding or lateral compression of the coil into one plane. A sharp tone minimum may be obtained and the telephone not be sensitive enough to detect it. A telephone that is tuned to the pitch of the induction coil is better, but the tune of the coil may change even though the spring of the vibrator is very stiff. A high frequency generator or Vreeland oscillator should not change in pitch, and is necessary for the greatest accuracy.

Since $\mathrm{I}^{\circ}$ rise in temperature causes an increase in electric conductivity of about 2.5 per cent, the conductivity cell must be placed in a water bath whose temperature is kept constant or observed very accurately at the moment of the determination.

If the electrodes are I $\mathrm{cm}$ apart and have I sq. $\mathrm{cm}$ surface the conductivity of the solution in reciprocal ohms is the specific conductivity. The specific conductivity divided by the concentration $(n)$ is called the molecular conductivity $(\lambda)$. The usual 
practice is not to measure the electrodes but to calibrate the cell with a known solution of $\mathrm{KCl}$.

It is found that the molecular conductivity of an electrolyte increases with dilution, or, in other words, the dissociation increases. At infinite dilution the dissociation would be complete, and is practically complete at .000 mol.

The question may be asked: Where does the electric charge of the ion come from? The study of radium has shown that the molecule contains a store of electricity but the positive charges exactly equal the negative charges and the molecule therefore is electrically neutral. These charges may exist free from molecules or ions and are then called electrons. It seems probable that negative electrons have no weight. A molecule of $\mathrm{NaCl}$ may be represented by an atom of $\mathrm{Na}$ and an atom of $\mathrm{Cl}$, each with a positive charge, connected by means of two negative electrons. When the molecule dissociates the negative electrons go with the $\mathrm{Cl}$ atom and give it an excess of one negative charge, whereas the $\mathrm{Na}$ atom has a positive charge.

If an electric current is passed through a solution, anions are deposited on the anode and cations on the cathode. It is necessary to pass 96500 coulombs of electricity ( $=$ I Faraday) in order to deposit I gram equivalent of ions on an electrode. If the ions remain on the electrodes as in electroplating, a gram molecule of the electrolyte is removed from the solution by the passage of 96500 coulombs, for a gram equivalent of anions is deposited on the anode and a gram equivalent of cations on the cathode. In any cross section of the solution, if $\mathrm{x}$ gram equivalents of anions is moving in one direction, $I-x$ gram equivalents of cations is moving in the opposite direction. Supposing that the cations move twice as fast as the anions, if we draw an imaginary cross section half way between the electrodes, twice as many cations will pass going in one direction as anions going in the opposite direction, or $2 / 3$ gram equivalent of cations move to the cathode and $1 / 3$ gram equivalent of anions to the anode. Around the cathode I gram equivalent of cations is removed by the electrode and $2 / 3$ is gained by migration, leaving a deficiency of $1 / 3$, whereas $1 / 3$ of anions are lost by migration, or, in other words, $\mathrm{T} / 3 \mathrm{~mol}$ electrolyte is lost. At the anode $2 / 3 \mathrm{~mol}$ electrolyte is lost. The change in concentration of the electrolyte at the 
electrode gives the transport number of the ion. By following the above reasoning backward, the ionic speed may be calculated (Hittorf).

These values of ionic speed are relative. The actual speed of ions is slow, as is shown by electrolyzing a solution containing colored anions around the cathode, and observing the time necessary for them to reach the anode.

The relative speeds of ions may be more conveniently determined by comparing the molecular conductivities of a series of salts that are completely dissociated. The molecular conductivity of a salt is proportional to the sum of the ionic speeds of anion and cation $(v+u)$. Thus, the ratio of the molecular conductivities of $\mathrm{NaCl}$ and $\mathrm{KCl}$ is the ratio of the ionic speeds of $\mathrm{Na}$ and $K$.

The relative ionic speeds at $18^{\circ}$ according to Kohlrausch (I907) are as follows:

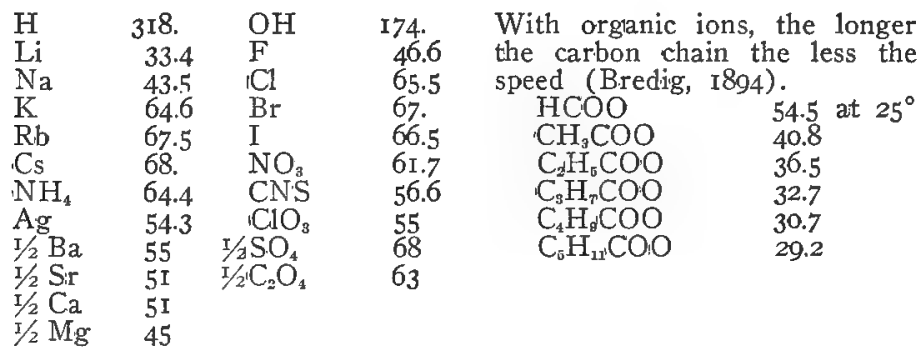

In general we may divide electrolytes into two classes, the strong and the weak. Strong electrolytes are greatly dissociated, whereas weak electrolytes are poorly dissociated. So-called nonelectrolytes may be to a minute extent dissociated. Water is a non-electrolyte; its ions are the most rapid, but it is poorly dissociated. Whereas the concentration of water is about 55 formal ( $55 \times 18 \mathrm{~g}$ per liter), only $10^{-7} \mathrm{~mol}$ is dissociated at $22^{\circ}$. [Owing to the partial association of $\mathrm{H}_{2} \mathrm{O}$ into $\left(\mathrm{H}_{2} \mathrm{O}_{2}\right)$ its concentration is less than $55 \mathrm{~mol}$.]

Strong electrolytes have not yet been brought entirely under the law of mass action. Weak electrolytes obey the law, and therefore their ions must obey it. Take, for example, the dissociation of acetic acid at different dilutions. The equation for 
this sort of phenomenon was found even before the law of mass action was so widely applied, and is known as Ostwald's dilution law.

If $a$ represents the degree of dissociation of such an electrolyte, of which one mol is dissolved in $\mathrm{v}$ liters, the concentration of the cations and of the anions is $\frac{\mathrm{a}}{\mathrm{v}}$, and of the undissociated molecules $\frac{\mathrm{I}-\mathrm{a}}{\mathrm{V}}$. Two reactions are going on at the same time, the dissociation of the molecules into ions and the recombination of the ions to form molecules. The rapidity of dissociation depends on the concentration of the molecules, $\frac{\mathrm{I}-\mathrm{a}}{\mathrm{V}}$, and the recombination on the product of the concentrations of the two classes of ions, $\frac{\mathrm{a}}{\mathrm{v}} \times \frac{\mathrm{a}}{\mathrm{v}}$, and at equilibrium, $\frac{\mathrm{a}}{\mathrm{v}} \times \frac{\mathrm{a}}{\mathrm{v}}=\mathrm{c} \frac{\mathrm{I}-\mathrm{a}}{\mathrm{v}}$, where $c$ is a constant, or $\frac{\frac{\mathrm{a}}{\mathrm{v}} \times \frac{\mathrm{a}}{\mathrm{v}}}{\mathrm{I}-\mathrm{a}}=\mathrm{c}$

by division we obtain $\frac{a^{2}}{v(r-a)}=c$. From this equation it is seen that ionization increases with dilution. Expressed in words, it means that the greater the dilution the farther removed the ions are from one another and the less chance they have of colliding with one another and recombining to form molecules, or the chance of their meeting is equal to the product of the concentration of the anions and the concentration of the cations.

This formula does not hold for the strong electrolytes, and Rudolphi (r895) and van't Hoff have applied the empirical formula, $\frac{a^{3}}{v\left(I-a^{2}\right)}=c$, to them. One reason that the strong electrolytes do not seem to obey the law of mass action may be that they form different hydrates at different dilutions. Some of these hydrates have been crystallized and others detected in other ways. Not only some molecules, but also all ions are sup- 
posed to be in the form of hydrates. Sulphuric acid forms the following hydrates (see Fig. 5) : ${ }_{5} \mathrm{H}_{2} \mathrm{SO}_{4} \cdot \mathrm{H}_{2} \mathrm{O},{ }_{3} \mathrm{H}_{2} \mathrm{SO}_{4} \cdot \mathrm{H}_{2} \mathrm{O}$, $\mathrm{H}_{2} \mathrm{SO}_{4} \cdot \mathrm{H}_{2} \mathrm{O}, \quad \mathrm{H}_{2} \mathrm{SO}_{4} \cdot 2 \mathrm{H}_{2} \mathrm{O}, \mathrm{H}_{2} \mathrm{SO}_{4} \cdot 3 \mathrm{H}_{2} \mathrm{O}, \mathrm{H}_{2} \mathrm{SO}_{4} \cdot{ }_{4} \mathrm{H}_{2} \mathrm{O}$ and $\mathrm{H}_{2} \mathrm{SO}_{4} \cdot \mathrm{I}_{2} \mathrm{H}_{2} \mathrm{O}$, at percentages $96,94,85,79,65,58$, and 30 of sulphuric acid (C. E. Davis, I9I5).

It should be emphasized that strong electrolytes exist chiefly as ions in the dilute solutions with which we have to deal in biochemistry. Take, for example, the neutralization of $\mathrm{HCl}$ with $\mathrm{KOH}$. Since $\mathrm{HCl}, \mathrm{KOH}$ and $\mathrm{KCl}$ take no part in the reaction and are practically never present, the reaction becomes $\mathrm{H}^{\bullet}+\mathrm{OH}^{\prime}=\mathrm{H}_{2} \mathrm{O}$. $\mathrm{Cl}^{\prime}$ and $\mathrm{K}^{*}$ are present in the same quantities before and after the reaction takes place. That the reaction is simply the formation of water from its ions is illustrated by the fact that no matter what strong base or acid is substituted for the above (at $20^{\circ}$ ), 13700 calories of heat are produced for I mol of acid and alkali used.

Ionization must be considered in studying the solubility of electrolytes. If we pass hydrochloric acid gas into a saturated solution of $\mathrm{NaCl}$, the salt will partially crystallize out. In a saturated solution of $\mathrm{NaCl}$, the product of the concentrations of $\mathrm{Na}^{\circ}$ and $\mathrm{Cl}^{\prime}$ is a constant, and when we add more $\mathrm{Cl}^{\prime}$ by the addition of $\mathrm{HCl}$, salt crystallizes out until the product assumes its original value. (This principle is used in the recrystallization of $\mathrm{NaCl}$ by the introduction of $\mathrm{HCl}$ gas into the saturated solution.)

Some electrolytes dissociate into three or more ions. They dissociate by steps, thus $\mathrm{K}_{2} \mathrm{SO}_{4}$ dissociates into $\mathrm{K}^{*}$ and $\mathrm{KSO}_{4}^{\prime}$ and the latter dissociates into $\mathrm{K}^{*}$ and $\mathrm{SO}_{4}{ }^{\prime \prime}$. In certain cases the first step may be complete and the second be incomplete under ordinary conditions. The dissociation of $\mathrm{H}_{2} \mathrm{CO}_{3}$ and $\mathrm{H}_{3} \mathrm{PO}_{4}$ will be considered in a later chapter.

In case one of the ions of an electrolyte is weak and the other strong hydrolysis may occur. $\mathrm{K}_{2} \mathrm{CO}_{3}$ dissociates into $\mathrm{K}^{*}$ and $\mathrm{KCO}_{3}{ }^{\prime}$. $\mathrm{K}$ ' combines with $\mathrm{OH}^{\prime}$ of the water and forms $\mathrm{KOH}$ while the other ion unites with $\mathrm{H}^{\circ}$ of water to form $\mathrm{KHCO}_{3}$. Since the $\mathrm{KOH}$ dissociates more $\mathrm{OH}$ ions than the bicarbonate produces $\mathrm{H}$ ions the reaction is alkaline. The further steps in this hydrolysis will be considered later. Hydrolysis is the union with the ions of water already present. When these are ex- 

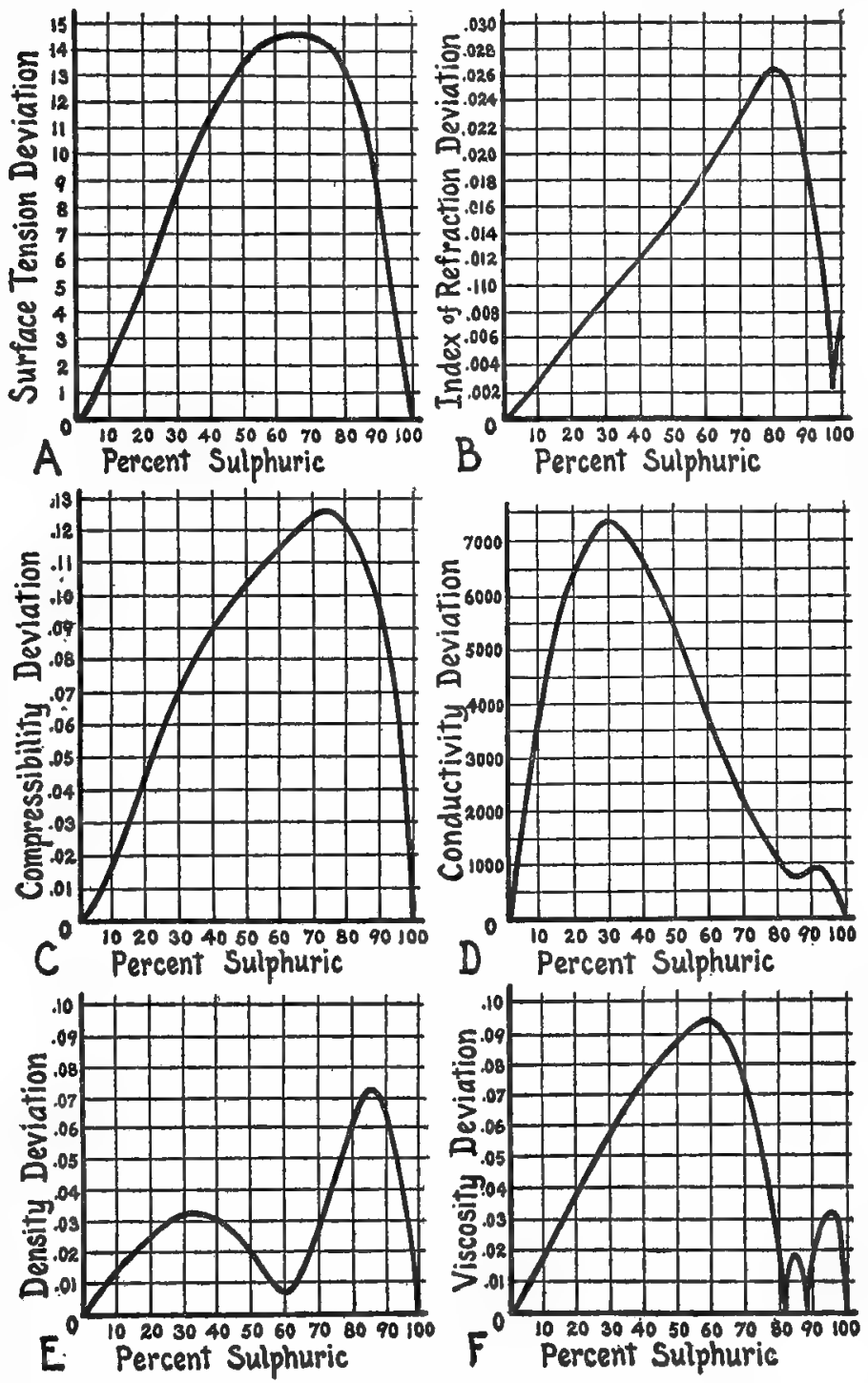

FIG. 5. Curves indicating the concentrations at which changes in the hydration of sulphuric acid occur (from Clarke Edwin Davis, IgI5). 
hausted more are formed, and the whole process is instantaneous in the above case. In this it is distinguished from the hydrolysis of proteins, for instance, in which the protein is boiled for a long time with acid or alkali, or hydrolyzed by means of enzymes. In the salt hydrolysis water is all that is necessary, but in the case of proteins the hydrolysis occurs with unmeasurable slowness in pure water.

Some substances may dissociate both $\mathrm{H}^{\bullet}$ and $\mathrm{OH}^{\prime}$, and are therefore called amphoteric electrolytes or ampholytes. The amino acids and proteins belong to this class. Their general formula is $\mathrm{NH}_{2} \mathrm{RCOOH}$, in which $\mathrm{R}$ represents a carbon chain (or a series of them connected by the amino and carboxyl groups). Some of these may be about neutral, but ustrally the acid or basic character predominates. The double nature of these substances is shown by their compounds. If $\mathrm{HCl}$ is added to egg albumin the latter no longer dissociates $\mathrm{H}$ ions, owing to the large number already in solution, but it continues to dissociate $\mathrm{OH}$ ions and as fast as these are formed their place in the albumin is taken by $\mathrm{Cl}$ ions, so that more $\mathrm{OH}$ ions continue to form and to combine with the $\mathrm{H}$ ions in solution. In this way Alb. $\mathrm{Cl}$ is formed, which dissociates into $\mathrm{Alb}^{\circ}$ and $\mathrm{Cl}^{\prime}$. If an electric current is passed through the solution the albumin will go to the cathode.

On the contrary, if $\mathrm{KOH}$ is added to an albumin solution Alb. $K$ will be formed by the displacement of the $H$ of the hydroxyl, and if an electric current is passed the albumin will go to the anode.

At a certain reaction of the solution the albumin will not migrate in an electric field, or if it does half will go toward the cathode and half toward the anode. This reaction is called the isoelectric point for that albumin. This subject will be continued in Chapter IV. 


\section{CHAPTER III}

\section{OSMOTIC PRESSURE}

There are some membranes that are freely permeable to water but impermeable to many dissolved substances, and are called semipermeable membranes. An example of such is found in one of Traube's (I867) membranes. If a solution of potassium ferrocyanide is allowed to come in contact with a solution of $\mathrm{CuSO}_{4}$, a membrane of copper ferrocyanide is formed, which is impermeable to potassium ferrocyanide, $\mathrm{CuSO}_{4}$ and their ions and to a certain degree to some other electrolytes such as $\mathrm{MgSO}_{4}$ and its ions. If this membrane is made in the form of a bag with $\mathrm{CuSO}_{4}$ on the inside and placed in pure water, so much pressure will be developed within it that it will burst. This pressure is called the osmotic pressure of the $\mathrm{CuSO}_{4}$ solution and can be measured if the membrane is strong enough. Membranes may be deposited in the pores of strong clay cups and thickened by driving in the mother substances with an electric current (H. N. Morse, I9I4). If such a cup is connected with a mercury or nitrogen manometer so that the osmotic pressure can be measured it is called an osmometer.

It was found by de Vries (1884), Pfeffer (1877) and others that I gram molecule ( $\mathrm{mol}$ ) of a non-electrolyte, such as dextrose, dissolved in water to make I liter has an osmotic pressure of 22.4 atmospheres at $0^{\circ}$. De Vries found that electrolytes departed from this rule, but Arrhenius (I887) showed that this discrepancy could be explained by his theory of electrolytic dissociation, since the ions exert osmotic pressure as well as the molecules, and hence ionization would increase osmotic pressure as the total osmotic pressure is the sum of the partial pressure of molecules + ions. Van't Hoff (1887) pointed out that osmotic pressure of ideal dilute solutions is the same quantitatively as gas pressure.

The formula for osmotic pressure of dilute ideal solutions is $\mathrm{pv}=\mathrm{RT}$, where $\mathrm{p}$ is the pressure in atmospheres, $\mathrm{v}$ is the vol- 


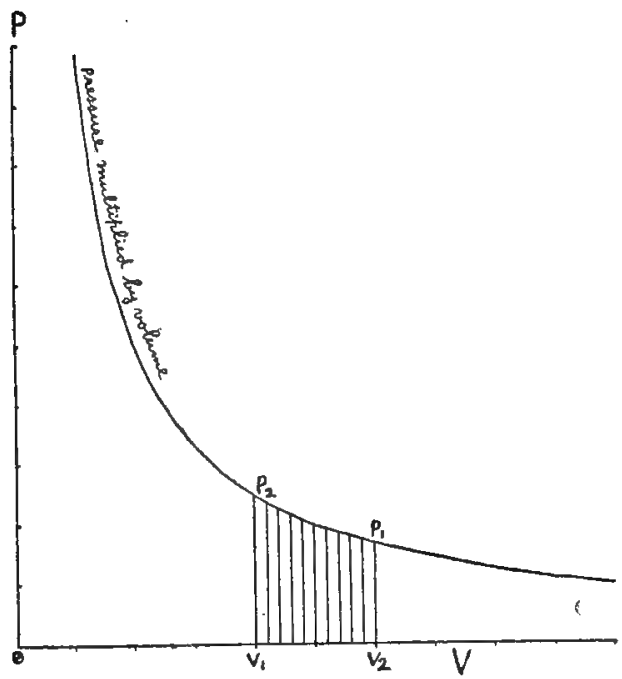

FIG. 6. Graph indicating the method of integrating the formula $P V=R T$ (from MRR).

ume in liters in which I mol solute is contained, $R=0.082 \mathrm{I}$, and $\mathrm{T}$ is the absolute temperature, in which zero is -273.090 on the centigrade scale (Fig. 6). Since the volume is the reciprocal of the concentration, the formula becomes $\frac{p}{c}=0.082 \mathrm{I} T$, where $\mathrm{c}$ is the molecular (molar) concentration. If the concentration is $\mathrm{I}$ and the temperature $=0^{\circ} \mathrm{C}=273$ abs., $\mathrm{p}=.082 \mathrm{I} \times 273$ $=22.4$. In other words, the osmotic pressure of a molar solution is 22.4 atmospheres at $0^{\circ}$. From this a method was developed of determining the extent of dissociation of an electrolyte from its osmotic pressure. If I $\mathrm{mol} \mathrm{KCl}$ is dissolved in so large a volume of water that it is completely dissociated it will exert double the pressure of I mol dextrose dissolved in the same volume, because the number of ions is double the number of molecules, and the formula becomes $\mathrm{pv}=2 \mathrm{RT}$. If the salt is partly dissociated a number somewhere between I and 2 must be used (say 1.5 ), then 50 per cent of the salt is dissociated.

The most accurate determinations of osmotic pressure have been done on sugars by $H$. N. Morse (I9I4) and Berkeley and Hartley (I904). Morse made some determinations on elec- 
trolytes, but finds that they increase the permeability of the membrane. The results of Morse do not exactly correspond to the formula, and he supposes this due to the fact that the sugar molecules take up so much room that the concentration of the water is reduced. By dissolving the sugar in I000 $\mathrm{g}$ of water instead of making a liter of solution, the formula was more closely followed. Such solutions are called weight normal solutions, but the water combined with the sugar molecules is not considered. According to Washburn (I915) each sugar molecule is combined with six molecules of water, which means that in a liter of a mol solution, $108 \mathrm{~g}$ of water per liter are taken from the solvent. The significance of the room occupied by the molecules is strikingly shown in solutions containing solutes of very high molecular weight. The osmotic pressure of a 30 per cent gum arabic solution is 4 atmospheres, whereas that of a 60 per cent solution is 48 atmospheres. If blood serum whose osmotic pressure is 6 atmospheres is concentrated to $1 / 4$ volume, its osmotic pressure is 35 atmospheres.

Owing to the difficulty of determining the osmotic pressure directly, it is usually calculated from the freezing point. An osmotic pressure of 22.4 atmospheres corresponds to a freezing point lowering $(\Delta)$ of $1.85^{\circ}$. The following conversion table was calculated by Harris and Gortner (I9I4) from the formula of Lewis (Igo8).

In determining the freezing point of aqueous solutions, the only advantage of the Beckmann thermometer is that it may be accessible. It has the disadvantage that it must be adjusted for aqueous solutions and that the zero point must be determined every year. The curvature and the temperature of the two mercury surfaces bordering on the vacuum are not always the same and hence the vapor pressure of the mercury at these two surfaces is not the same, and consequently mercury distils over from one surface to the other. The Heidenhain thermometer has no adjustment to cause this trouble. It is a simple thermometer graduated in hundredths of a degree from about $+0.5^{\circ}$ to $-5^{\circ}$. The Burian-Drucker (IgIo) thermometer is designed for fluids of which only $1.5 \mathrm{cc}$ may be available. Since it has a smaller bulb and consequently shorter stem, it is graduated in fiftieths of a degree. The thermometer should be kept in ice water several hours before the determination. 
Table of Osmotic Pressures in Atmospheres for Depression of the FreEzing PoInt.

\begin{tabular}{|c|c|c|c|c|c|c|c|c|c|c|}
\hline \multirow{2}{*}{$\Delta$} & \multicolumn{10}{|c|}{ Hundredths of degrees, centigrade } \\
\hline & 0 & 1 & 2 & 3 & 4 & 5 & 6 & 7 & 8 & 9 \\
\hline 0.0 & 0.000 & o.I 2 I & $0.24 \mathrm{I}$ & 0.362 & 0.482 & 0.603 & 0.724 & 0.844 & 0.965 & $x .085$ \\
\hline o.I & I.206 & 1.327 & 1.447 & I. 568 & I. 688 & 1.809 & 1.930 & 2.050 & $2.17 \mathrm{I}$ & 2.291 \\
\hline 0.2 & 2.412 & 2.532 & 2.652 & 2.772 & 2.893 & 3.014 & 3.134 & 3.255 & 3.375 & 3.496 \\
\hline 0.3 & 3.616 & 3.737 & 3.857 & 3.978 & 4.098 & 4.219 & $4 \cdot 339$ & 4.459 & 4.580 & 4.700 \\
\hline 0.4 & $4.82 \mathrm{I}$ & 4.941 & 5.062 & 5.182 & 5.302 & 5.423 & 5.543 & 5.664 & 5.784 & 5.904 \\
\hline 0.5 & 6.025 & 6.145 & 6.266 & $6.3^{86}$ & 6.506 & 6627 & 6.747 & 6.867 & 6.988 & 7.108 \\
\hline 0.6 & 7.229 & 7.349 & 7.469 & 7.590 & 7.710 & 7.830 & 7.951 & $8.07 \mathrm{I}$ & $8.19 \mathrm{I}$ & 8.312 \\
\hline 0.7 & $8.43^{2}$ & 8.552 & 8.672 & 8.793 & 8.913 & 9.033 & 9.154 & 9.274 & 9.394 & 9.514 \\
\hline 0.8 & 9.635 & 9.755 & 9.875 & 9.995 & 10.12 & 10.24 & 10.36 & 10.48 & 10.60 & 10.72 \\
\hline 0.9 & 10.84 & 10.96 & I 1.08 & II.20 & II $3^{2}$ & I I . 44 & I I. 56 & 11.68 & 11.80 & 11.92 \\
\hline I.O & I 2.04 & I 2.1 6 & I 2.28 & 12.40 & $12.5^{2}$ & 12.64 & 12.76 & 12.88 & 13.00 & 13.12 \\
\hline I.I & 13.24 & $13.3^{6}$ & I 3.48 & 13.60 & 13.72 & 13.84 & 13.96 & 14.08 & 14.20 & 14.32 \\
\hline 1.2 & I 4.44 & I 4.56 & 14.68 & I 4.80 & 14.92 & 15.04 & 15.16 & 15.28 & 15.40 & 15.52 \\
\hline 1.3 & 15.64 & I 5.76 & I5.88 & 16.00 & 16.1 2 & 16.24 & 16.36 & I 6.48 & I 6.60 & 16.72 \\
\hline $\mathrm{I} .4$ & 16.84 & 16.96 & $\mathrm{I} 7.08$ & 17.20 & $17.3^{2}$ & 17.44 & I 7.56 & 17.68 & 17.80 & 17.92 \\
\hline 1.5 & I8.04 & 18.16 & I 8.28 & 18.40 & 18.52 & 18.64 & 18.76 & 18.88 & 19.00 & 19.12 \\
\hline $1 . \overrightarrow{6}$ & 19.24 & $19.3^{6}$ & 19.48 & 19.60 & 19.72 & 19.84 & 19.96 & 20.08 & 20.20 & 20.32 \\
\hline 1.7 & 20.44 & $20.5^{6}$ & 20.68 & 20.80 & 20.92 & 21.04 & 21.16 & 21.28 & 21.40 & $2 \mathrm{I} .52$ \\
\hline 1. 8 & 21.64 & $21.7^{6}$ & 21.88 & 22.00 & 22.12 & 22.24 & 22.36 & $22.4^{8}$ & 22.60 & 22.72 \\
\hline 1.9 & 22.84 & 22.96 & 23.08 & 23.20 & 23.32 & 23.44 & 23.56 & 23.68 & 23.80 & 23.92 \\
\hline 2.0 & 24.04 & 24.16 & 24.28 & 24.40 & 24.52 & 24.63 & 24.75 & 24.87 & 24.99 & $25.1 \mathrm{II}$ \\
\hline 2.I & 25.23 & 25.35 & 25.47 & 25.59 & $25.7 \mathrm{I}$ & 25.83 & 25.95 & 26.07 & 26.19 & 26.31 \\
\hline 2.2 & 26.43 & 26.55 & 26.67 & 26.79 & 26.91 & 27.03 & 27.15 & 27.27 & 27.39 & 27.51 \\
\hline 2.3 & 27.63 & 27.75 & 27.87 & $27.99^{\circ}$ & 28. I I & 28.23 & 28.34 & 28.46 & 28.58 & 28.70 \\
\hline 2.4 & 28.82 & 28.94 & 29.06 & 29.18 & 29.30 & 29.42 & 29.54 & 29.66 & 29.78 & 29.90 \\
\hline 2.5 & 30.02 & 30.14 & 30.26 & $130.3^{8}$ & 30.50 & 30.62 & 30.74 & 30.86 & 30.98 & 31.09 \\
\hline
\end{tabular}

The solution to be frozen is poured into a test tube having a double bored cork. This tube is sometimes provided with a side neck through which a small crystal of ice may be dropped to start the freezing as soon as the temperature reaches the freezing point and thus prevent undercooling. The test tube is fitted into a larger test tube and the space between them left full of air or filled with alcohol to prevent the condensation of moisture which would obscure observation. The freezing mixture is usually crushed ice or snow and salt, but this interferes with observation, and ether through which air is sucked is preferable.

The determination of the freezing point is as follows. About 22 cc of the solution is poured into a test tube, and the bulb of the thermometer and a platinum stirrer lowered into it through separate holes in the stopper. The test tube is cooled nearly to 
the right temperature by immersing it in ice and salt; it is then wiped dry and pushed through the bored cork of a larger test tube and returned to the freezing mixture. The thermometer should not touch the wall of the inner test tube, and this in turn should not touch the outer test tube, as the local cooling thus produced might cause a local freezing before the entire solution reached the freezing point. The solution is stirred until freezing commences. The temperature will fall below the freezing point and when the first ice separates suddenly rise to the freezing point. This undercooling may be lessened by having the freezing mixture only slightly below the freezing point of the solution or by dropping in a crystal of ice to start the freezing at the proper moment. Since the sudden separation of ice that occurs before the definitive freezing point is reached concentrates the remaining solution, the freezing point lowering is too great and should be decreased I/80 for every degree of undercooling.

Although the cryoscopic method is very simple in principle, it is only by the most painstaking performance that an accuracy of $.001^{\circ}$ is obtained. Since a $\Delta$ of $1.85^{\circ}$ corresponds to an osmotic pressure of 22.4 atmospheres, $.001^{\circ}$ represents a pressure of .0912 atmospheres or $9.2 \mathrm{~mm}$ of mercury, and is very serious when small differences in osmotic pressure are to be measured. It should be remembered that the cryoscopic method determines the osmotic pressure at the freezing point of the solution, and this should be reduced to the desired temperature by correcting for the effect of the temperature change on the electrolytic dissociation and osmotic pressure.

A method for determining the freezing point of small amounts of solution, devised by Barger ( 1904 ), has been called a microtensimeter, but it is in reality an osmometer in which air forms the semipermeable membrane. It consists of a capillary tube that can be observed under a microscope having an ocular micrometer. The osmotic pressure of the solution is approximately known from previous data or a preliminary test. A graded series of solutions is prepared, the extremes of which will fall on the two sides of the osmotic pressure of the unknown. Drops of the unknown solution alternating with the known solutions are drawn up into the tube and their lengths measured. After an interval of time their lengths are again measured and the drop 
of unknown that has not changed in length found; its osmotic pressure is between those of the graded solutions on each side of it. One cc of the unknown is sufficient and hence the method is useful in biological work. This method has the advantage that it measures the osmotic pressure at any desired temperature. The chief error is in the mixing of the drops, caused by the adhesion of a surface film to the glass and the transfer of fluid from one drop to another in this film.

The way in which osmotic pressure may do work in the organism is best illustrated in plants. The plant cell is limited by the plasma membrane which is impermeable to some dissolved substances. Surrounding the plasma membrane is the cellulose cell wall. If the cell is placed in water the osmotic pressure of its interior causes it to swell, and the plasma membrane is pressed against the cellulose cell wall and tightens it or makes the cell turgid. This turgidity (turgor) is what gives stiffness to herbaceous plants, lacking which the plant is said to be wilted. The osmotic pressure of many plant cells is a number of atmospheres, and the cell wall has to be very strong in order to withstand the pressure. The extent of this pressure is realized when the roots of trees growing between rocks separate the rocks by the osmotic pressure exerted.

The cell wall of a young plant cell is very thin and is stretched by the osmotic pressure as the cell grows. Some mechanism must be provided for getting the osmotic substance into the cell, before it can stretch the cell and cause growth. In the green plant cell the osmotic substances are manufactured with the aid of sunlight out of $\mathrm{H}_{2} \mathrm{O}$ and $\mathrm{CO}_{2}$, to which the cell is freely permeable. The colorless cells absorb certain organic substances secreted by the green cells, transforming them into other substances to which the plasma membrane is impermeable, and which therefore accumulate in the cells and cause the osmotic pressure. Though these osmotic substances are not in all cases definitely known, tannic and oxalic acid and their salts have been postulated. The sugars are supposed by some investigators to be the nutritive substances passed from the green to the colorless cells, meaning that both kinds of cells are permeable to sugar. Overton, however, claims that plant cells are impermeable to sugar 
and hence any contained sugar could aid in maintaining the turgor.

A solution whose osmotic pressure is lower than that of the cell is said to be hypotonic, and one whose pressure is greater, hypertonic. The osmotic pressure of many animal cells is very near that of the medium, or the fluid which bathes them. When the medium is hypotonic the cell swells, when hypertonic it shrinks until equilibrium is established. Since animal cells are not surrounded by very strong cell walls, they burst or swell when placed in pure water. But some animal cells are impermeable to water, and consequently are not affected by changes in the medium.

Fresh water protozoa are prevented from bursting by the activity of the contractile vacuole. Zuelzer (1907) acclimatized Ameba verrucosa to sea water and the contractile vacuole beat more and more slowly and finally disappeared in $1 / 2$ sea water. The few marine protozoa that have contractile vacuole are parasitic and hence not in pure sea water and the pulsations of the vacuoles are much slower than is the case with fresh water forms.

Most of the cells in the mammalian, reptilian and avian bodies are bathed by fluids whose osmotic pressure is practically constant. One of these fluids is the blood. Its osmotic pressure at body temperature is about 8 atmospheres $\left(6.7\right.$ at $\left.0^{\circ}\right)$, its freezing point being about $-56^{\circ}$. This corresponds to about Io per cent (.3 molecular) cane sugar or .95 per cent $\mathrm{NaCl}$ solution (.I6 mol). See Fig. 30, Chapter XV.

More minute data on osmotic pressure will be considered in Chapter XV. 


\section{CHAPTER IV}

\section{HYDROGEN AND HYDROXYL ION CONCENTRATION AND $\mathrm{CO}_{2}$ PRESSURE}

Water is an electrolyte and dissociates into $\mathrm{H}$ and $\mathrm{OH}$ ions, which are therefore equal in number. At $22^{\circ}$ or $23^{\circ}$ $\mathrm{H}^{*}=\mathrm{OH}^{\prime}=\mathrm{IO}^{-7}$, or $\mathrm{H}^{\circ} \times \mathrm{OH}^{\prime}=\mathrm{IO}^{-14}$. If we increase $\mathrm{H}^{*}$ by adding acid, $\mathrm{OH}^{\prime}$ decreases, because $\mathrm{H}^{*} \times \mathrm{OH}^{\prime}$ remains equal to $\mathrm{IO}^{-14}$. Conversely, if we add alkali, $\mathrm{H}^{*}$ decreases, and hence we may estimate the acidity or alkalinity of a solution by determining the $\mathrm{H}^{*}$ concentration. The product of the $\mathrm{H}$ and $\mathrm{OH}$ ion concentrations is $\mathrm{IO}^{-14}$ and the - logarithm is $\mathrm{I} 4$ (and is designated $-\log \mathrm{K}_{\mathrm{w}}$ ). The following table gives the values of $-\log \mathrm{K}_{\mathrm{w}}$ for various temperatures, calculated thermodynamically from the results of Lorenz and Boehi (I909) at I $8^{\circ}$, and which agree with those of Sorensen, Michaelis and some others within about one degree.

\begin{tabular}{|c|c|c|c|c|c|c|c|c|c|c|c|}
\hline \multicolumn{2}{|c|}{$t^{\circ}-\log K w$} & \multicolumn{2}{|c|}{$\mathrm{t}^{\circ}-\log \mathrm{Kw}$} & \multicolumn{2}{|c|}{$t^{\circ}-\log K w$} & \multicolumn{2}{|c|}{$\mathrm{t}^{\circ}-\log \mathrm{Kw}$} & \multicolumn{2}{|c|}{$t^{0}-\log K w$} & \multicolumn{2}{|c|}{$t^{\circ}-\log K w$} \\
\hline 18 & 14.142 & 22 & I 4.004 & 26 & $13.86 \mathrm{r}$ & 30 & I 3.733 & 34 & $13.6 \mathrm{II}_{1}$ & 38 & 13.513 \\
\hline 19 & 14.108 & 23 & 13.960 & 27 & I 3.828 & $3 I$ & 02 & 35 & $8 I$ & 39 & 13.475 \\
\hline 20 & 14.073 & 24 & 13.9 & 28 & I 3.796 & 32 & $13.67 \mathrm{r}$ & 36 & 13.561 & 40 & I 3.446 \\
\hline 21 & 14.038 & 25 & I 3.894 & 29 & 13.765 & 33 & 13.641 & 37 & 13.532 & & \\
\hline
\end{tabular}

In order to save space it is better to express $\mathrm{H}^{\circ}$ concentration as the minus logarithm $(\mathrm{PH})$. Thus if $\mathrm{H}^{*}$ concentration $=1 \mathrm{O}^{-7}$, $\mathrm{PH}=7$ (Sorensen I909).

In order to understand the method by which the $\mathrm{H}$ ion concentration is determined we must first consider the theory of concentration cells and electrode potentials. The fact that an electric potential difference is produced at the surface of contact of the metal with a solution is explained as follows: We know that the ions of metals are soluble because they are produced by the solution of metallic salts. There is also a store 
of electricity in the metal for charging the ions as illustrated by the behavior of radioactive metals. If a piece of metal is placed in pure water it gives off ions, but these ions taking positive charges from the metal leave the metal negative, and when the attraction of the negative metal for the positive ions equals the diffusion pressure ( $=$ osmotic pressure) of the ions no more ions will be given off. If instead of placing the metal in pure water we place it in a solution containing ions of the metal, the metal will give out a less number of ions and will become less negative. If the concentration of the metallic ions in the solution is increased, a point will be reached at which the metal will give out no ions and hence remain electrically neutral, and if the ionic concentration of the solution is still further increased ions will be deposited on the metal and give it a positive charge. If a piece of metal is placed in a solution containing I gram equivalent of its ions per liter it may become negative or positive according to the metal used. The following table is prepared in this way, but the neutral point (taken arbitrarily) is not the potential of the earth but the potential of a hydrogen electrode in a gram equivalent solution of hydrogen ions (see Abegg Auerbach \& Luther, I9I I). The potential that the electrode assumes is called the electrolytic solution tension.

\section{Electrolytic Solution Tension.}

Potential of metal (or non-metal) when immersed in a normal solution of its ions at $25^{\circ}$, as measured against a normal hydrogen electrode. Some American writers use reverse signs $(+,-)$.

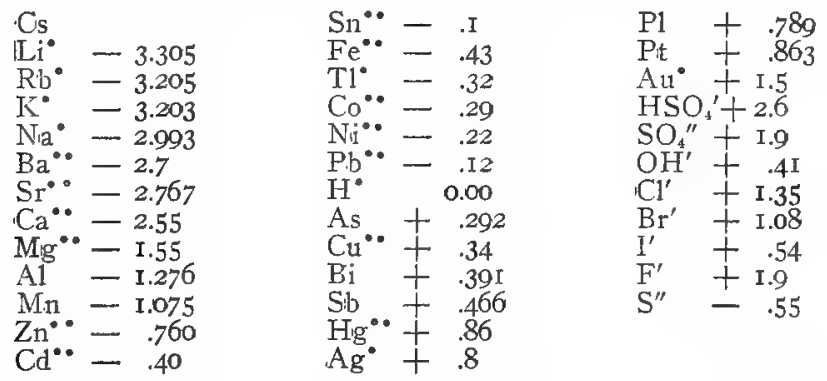

It may be seen from the table that the baser metals have a greater tendency to dissolve and produce ions than the noble metals.

Electrolytic solution tension is reduced by certain impurities 
in solution as follows, beginning with least effective: mannite $<$ glycerine < glycol < meth. alc. <eth. alc. < acetone < prop. alc. <ether. (Abl, I907.)

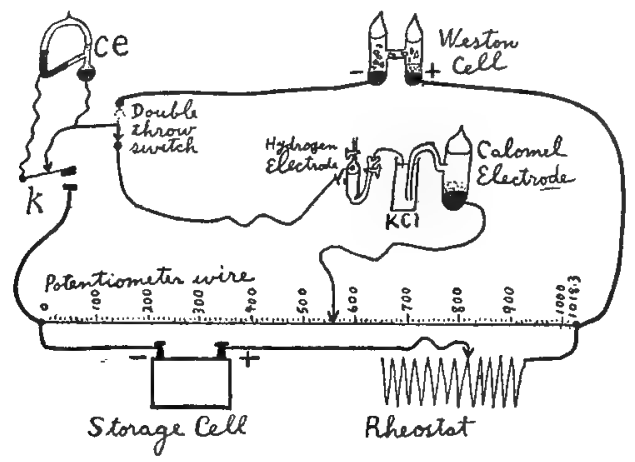

FIG. 7. Method of connecting the apparatus for determining hydrogen ion concentration (from MRR).

Determination of Hydrogen Ion Concentration with Hydrogen Electrodes

The apparatus, Fig. 7, consists of a hydrogen electrode, a vial, filled with a saturated solution of $\mathrm{KCl}$, and electrolytically connected with the hydrogen electrode, a calomel electnode electrolytically connected with the vial by means of a syphon, a capillary electrometer, ce, with compound reading microscope,

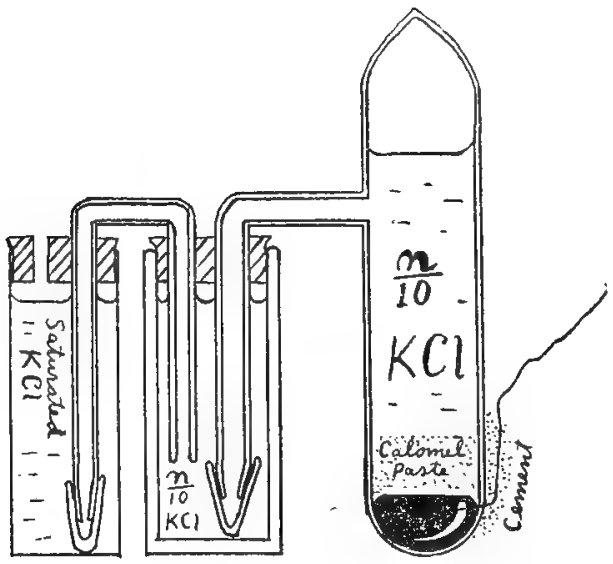

FIG. 8. Tenth normal calomel electrode (from MRR). 
a double throw switch, a knife switch (not shown), a telegraph $\mathrm{key}, k$, a potentiometer wire with two (or three) movable contacts, a Weston cell and a storage cell. The pieces of apparatus are connected by means of copper wires as shown in Fig. 7 .

The o.I n calomel electrode, Fig. 8, is a test tube with syphon side neck, and a platinum wire or foil fused in the bottom. The platinum is plated or covered with pure mercury. Over the mercury is placed a layer of calomel (made by precipitation and washed repeatedly with a .I n solution of $\mathrm{KCl}$ ). The electrode is filled with the solution of $\mathrm{KCl}$ and the top closed so that the solution will not run out the syphon. The end of the syphon is closed with some porous material or an ungreased ground glass stopper or stopcock and immersed in a saturated solution of $\mathrm{KCl}$, into which the stopcock of the hydrogen electrode also is dipped. An improvised electrode is shown in Fig. 9 (saturated type).

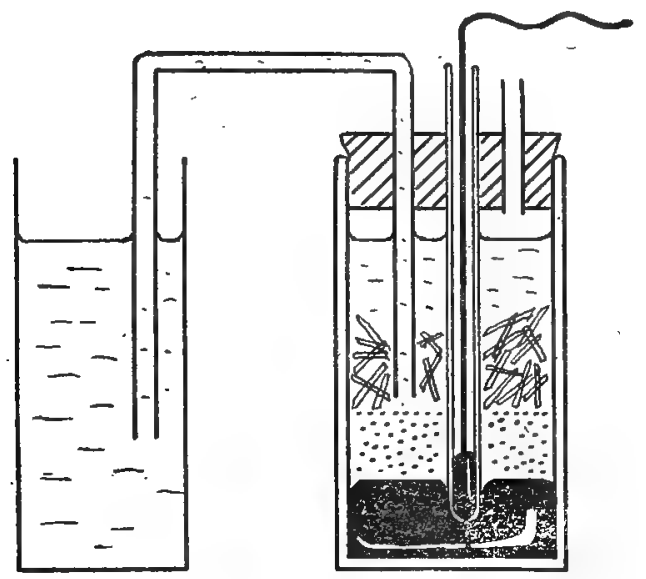

FIG. 9. Saturated calomel electrode (from MRR).

The capillary electrometer may be bought sealed or it may be made in the form shown in Fig. 7. Pure mercury is poured in so as to make contact with the fused in platinum wires in the wide and narrow arms, and about 30 per cent $\mathrm{H}_{2} \mathrm{SO}_{4}$ (by weight) is added until the capillary tube is filled. For accurate work this capillary must be fine as thermometer tubing and thin walled, so that higher power lenses may be used. The electrometer is 
clamped on the stage of a tilted back microscope. If much current is run through the electrometer, a drop of mercury must be allowed to pass from the capillary into the large arm.

The telegraph key short circuits the electrometer when not in use, and if a simple key is used instead, a short circuiting switch must be added.

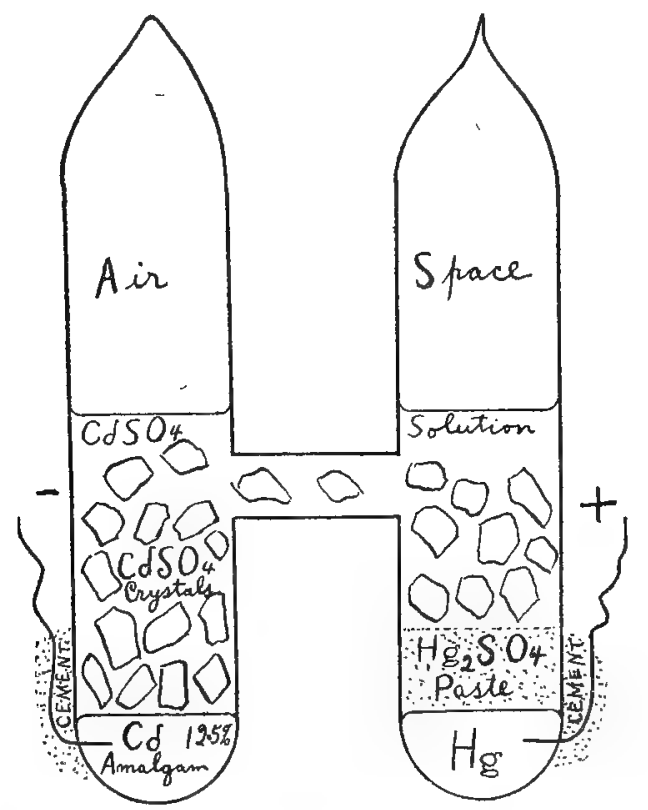

FIG. Io. Weston standard cell, saturated type (from MRR).

It is not usually economical to make a Weston cell (Fig. IO) if only one is needed and the materials are not already at hand. Directions for making it are given in Ostwald-Luther, "Messungen," I9IO. Its voltage should be I.0I83 at $20^{\circ}$. If it is short circuited it will not recover for several days.

The potentiometer should have a high electrical resistance in order to prevent the storage cell from running down rapidly, and to reduce errors due to contact resistances within itself.

If a standard potentiometer or one like Fig. I I is not available a No. 36 nichrome wire stretched over a IOI8.3 millimeter scale may be used, but it must be calibrated as the meter wire is in Ostwald-Luther, "Messungen," I9ro. 


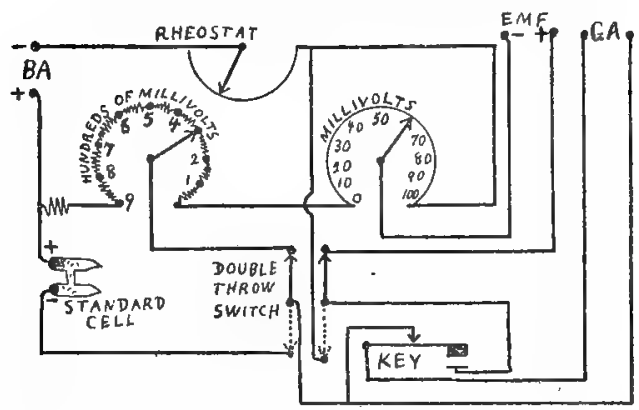

FIG. II. Potentiometer and standard cell combined (L and N) from MRR.

The principle of the potentiometer is as follows: Suppose we have a wire stretched over a scale divided into equal divisions, and an electric current flowing through the wire so that each division has a fall of potential of one millivolt. If we lead off a shunt circuit from two points on the wire ro divisions apart through an electrometer, a current of Io millivolts will flow through the electrometer. Interpose in the shunt circuit an emf of Io millivolts in a direction opposite to the current in the shunt, no current will flow through the shunt, and there will be no deflection in the electrometer. Interpose an unknown emf in the shunt and move the contacts on the potentiometer wire until no deflection is seen in the electrometer, and the voltage of the unknown is equal to the number of divisions between the contacts of the shunt leads, in millivolts. In order to standardize our current, we reverse this process by placing a known emf (Weston cell) in the shunt and then changing the total length of the wire until each division equals a millivolt.

The hydrogen electrode is essentially a metal electrode saturated with hydrogen. Platinum absorbs hydrogen greedily especially when in the finely divided state known as platinum black, and is excellent for the surface of the electrode. But it is better to make the interior of gold so that it will not absorb so much hydrogen and take it away from the surface. The gold is covered with platinum black as in platinizing conductivity electrodes, but it is not necessary to remove $\mathrm{Cl}_{2}$ by $\mathrm{H}_{2} \mathrm{SO}_{4}$ and electrolysis, as the electrode is never used as anode and no $\mathrm{Cl}_{2}$ is formed. In order to keep the electrode saturated with hydro- 
gen while it is immersed in the fluid to be investigated, special vessels are necessary, but these are usually inseparably connected with the electrode and included under the term.

Blood requires more precautions than any other biological fluid when investigated with the hydrogen electrode. When success has been achieved with it, the same technique and apparatus will serve for any other biological fluid with slight omissions and modifications for expediency. Though many forms of electrodes have been described, but four points in the technique need be emphasized: First, reduce the layer of blood over the platinum as much as possible so that hydrogen can get to the platinum (Michaelis "Wasserstoffionenkonzentration," I9I4). Second, shake the hydrogen with another sample of the same blood before passing it into the electrode, otherwise $\mathrm{CO}_{2}$ will pass from the blood into the hydrogen and leave the blood more alkaline (Hasselbalch, I9I3). Third (not absolutely necessary), remove the erythrocytes. Otherwise the oxygen they contain will combine with the hydrogen in the platinum and reduce its concentration (Milroy, I9I4). Fourth, connect the apparatus so that the blood is never exposed to the air and $\mathrm{CO}_{2}$ lost (McClendon I9I6 a).

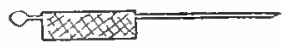

FIG. I2. Hollow needle for drawing blood (from JBC).

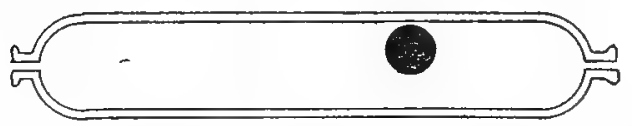

FIG. I3. Tube for collecting, defibrinating and centrifuging blood without exposure to air. The ends are fitted with rubber tubes (from MRR).

The following apparatus is the first to include all of these points (McClendon \& Magoon, I916).

The blood is drawn through a hollow needle, Fig. I2, connected by rubber tubing to the Io $\mathrm{cc}$ defibrination tube, Fig. I3, and defibrinated with the lead ball with the exclusion of air. This tube is then placed in the centrifuge and the corpuscles precipitated. The lower end is connected to a mercury tube and funnel, with the exclusion of air, and the upper end is connected to the Fresenius cock $a$ of the electrode, Fig. I4, that has already been rinsed with distilled water and filled with pure hydrogen. Rais- 


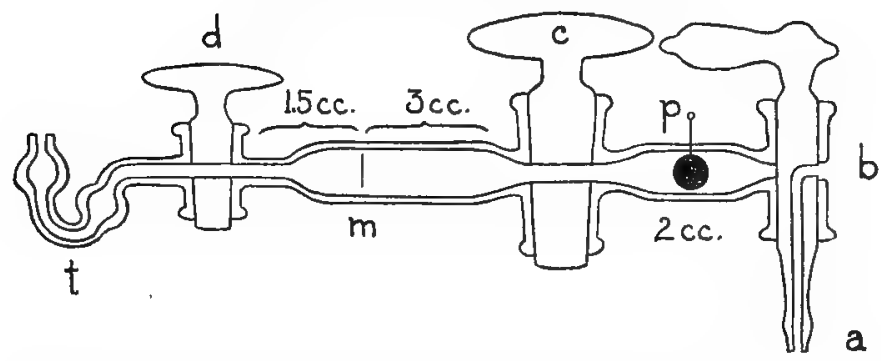

Fig. I4. Hydrogen electrode for fluids containing $\mathrm{CO}_{2}$. Hydrogen is shaken with one portion of the fluid in the large compartment and passed into another portion of the fluid in the small compartment without exposure to air (from JBC).

ing the mercury funnel causes the serum to rise in the cock and wash out the air, and when this is done the cock is turned (the two others likewise) so that the serum enters the electrode, the hydrogen bubbling out through the water in the trap, $t$. The $2 \mathrm{cc}$ chamber is completely filled with serum, the $4.5 \mathrm{cc}$ chamber filled until it contains only $\mathrm{I} .5 \mathrm{cc}$ of hydrogen and all of the cocks closed. The electrode is shaken or inverted 200 times, to bring the hydrogen to equilibrium as regards $\mathrm{CO}_{3}$. The middle cock is now opened and the electrode held vertically or shaken so that the hydrogen displaces $\mathbf{I} .5 \mathrm{cc}$ serum from the smaller cliamber. In passing from the large to the small chamber, the hydrogen is in contact with the returning serum spread in a thin film, and so completes $\mathrm{CO}_{2}$ equilibrium. The middle cock is closed and the electrode shaken or inverted 200 times, connected to the potentiometer by means of a wire hooked into the platinum loop, and to the calomel electrode by dipping the Fresenius cock into the vial filled with a saturated solution of $\mathrm{KCl}$. Clark and Lubs (I9I6) state that it is better to have the $\mathrm{KCl}$ meet the serum in a wide tube and make this contact immediately before taking the reading. I have not found this precaution necessary for an accuracy of I millivolt. If desired, the precaution may be taken by having the opening, a, Fig. I4, wide, lubricating this stopcock with serum before filling the electrode and leaving the outlet filled with serum after filling the electrode. The temperature of the room is controlled or at least noted. A convenient thermoregulator for the room is shown in Fig. 15. The first 


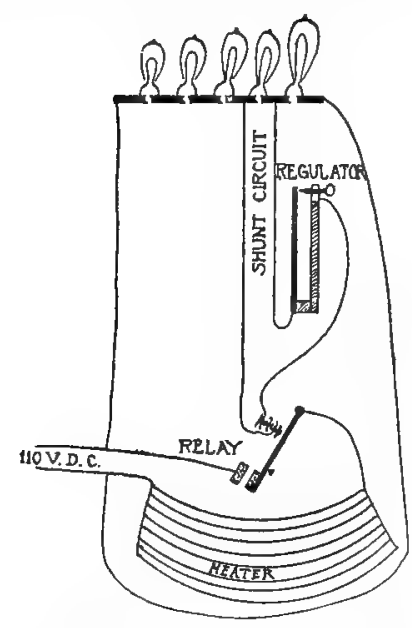

FlG. 15. Thermoregulator for maintaining constant temperature in a room. The regulator is of strips of invar and brass welded together, and the heater is of nichrome wire strung through the air. An electric fan is necessary (from JBC).

reading on the potentiometer is correct provided the electrode is the temperature of the room. If more readings are taken the electrode should be shaken or turned so as to splash a fresh layer of serum over the platinum. The efficiency of this electrode is shown by the fact that successive readings during one hour (or even forty-eight hours) are the same within I millivolt. The electrode is cleaned with potassium bichromate in sulphuric acid and left filled with distilled water. Before use, it is replatinized a few seconds, rinsed thoroughly with distilled water, the Fresinius cock lubricated with serum or $\mathrm{KCl}$ solution and the electrode filled with pure hydrogen. Venous blood may be used in the electrode instead of serum, provided hirudin or oxalate are used to prevent coagulation.

If it is desired to measure the $\mathrm{PH}$ of the serum at a known $\mathrm{CO}_{2}$ pressure, the electrode is sealed to a Hemphill burette. Since $\mathrm{CO}_{2}$ pressures above to per cent of an atmosphere are not desirable, the Ioo cc burette may be shortened, and only Io cc made narrow and graduated as in Fig. I6. This burette may be called a tonometer, since the pressure of $\mathrm{CO}_{2}$ in atmospheres is measured 


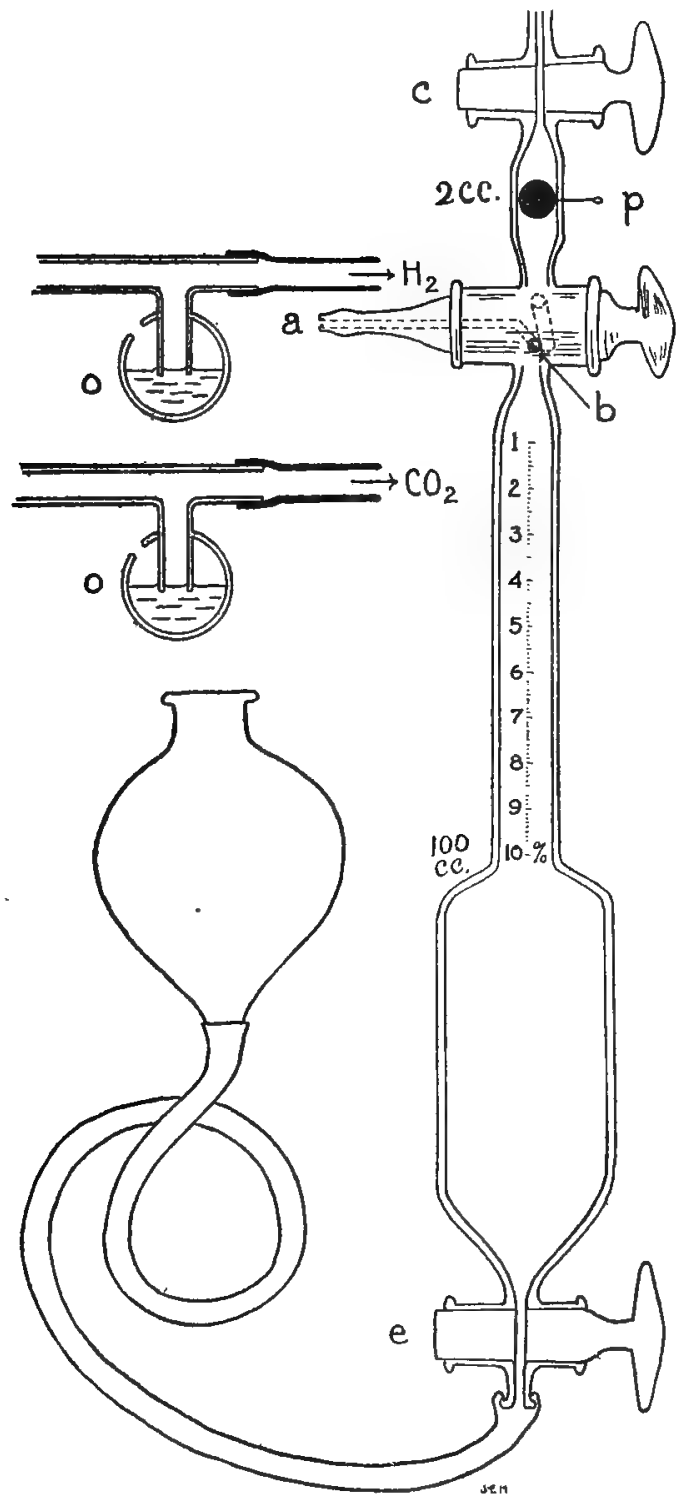

FIG. 16. Combined tonometer and hydrogen electrode. The fluid is passed from the $2 \mathrm{cc}$ electrode into the $100 \mathrm{cc}$ tonometer and back into the $2 \mathrm{cc}$ electrode (from JBC). 
by it. The electrode is first filled with serum through $a$. The apparatus is set up as in the figure, and the tonometer filled with mercury by raising the mercury funnel. The connections with the $\mathrm{H}_{2}$ and $\mathrm{CO}_{2}$ generators are provided with side tubes with traps, $o$, to make sure that the gas issuing from them never varies more than about a millimeter of water from atmospheric pressure. The three way cock, $a$, is connected with the $\mathrm{CO}_{2}$ generator, its outlet washed through the opening, $b$, and the cock turned so as to admit $\mathrm{CO}_{2}$ into the tonometer while the funnel is being carefully lowered. When the right per cent of $\mathrm{CO}_{2}$ is read off on the graduations the lower cock, $e$, and three way cock, $a$, are closed. Hydrogen is admitted in the same way as was the $\mathrm{CO}_{2}$, in order to fill the tonometer with gas at atmospheric pressure, and the lower cock, $e$, closed. If it is desired to make gas mixtures containing less than I per cent $\mathrm{CO}_{2}$, for example 0.03 per cent $\mathrm{CO}_{2}$, first make $\mathrm{I}$ per cent $\mathrm{CO}_{2}$, mix by allowing to stand or inverting the apparatus, and expel all but $3 \mathrm{cc}$ by raising mercury funnel, then fill with hydrogen. The apparatus is removed, the three way cock, $a$, is so turned as to connect the tonometer with the electrode, the serum shaken down into the tonometer and rotated fifteen minutes. One half $\mathrm{cc}$ of serum is shaken back into the electrode, connections made and reading taken. If it is desired to use another $\mathrm{CO}_{2}$ pressure on the same serum, all of the serum is shaken into the electrode and the process repeated. By measuring the $\mathrm{PH}$ of the serum at $\mathrm{CO}_{2}$ tension of the alveolar air, the PH of the arterial blood may be obtained. It is not always possible to distinguish between the $\mathrm{PH}$ of venous and of arterial blood, because the slight difference may come within the limit of error of the method, but since the $\mathrm{PH}$ of arterial blood is more constant than that of venous blood, the significance of this procedure is obvious.

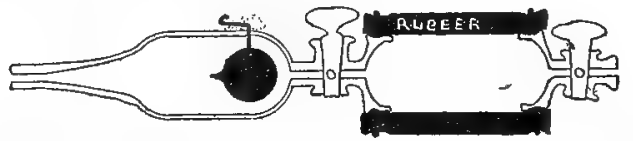

FIG. I7. Hydrogen electrode for $\mathrm{CO}_{2}$-free fluids (from MRR).

A simple hydrogen electrode for small quantities of fluid is shown in Fig. I7. If used for titrations the rubber bulb may 


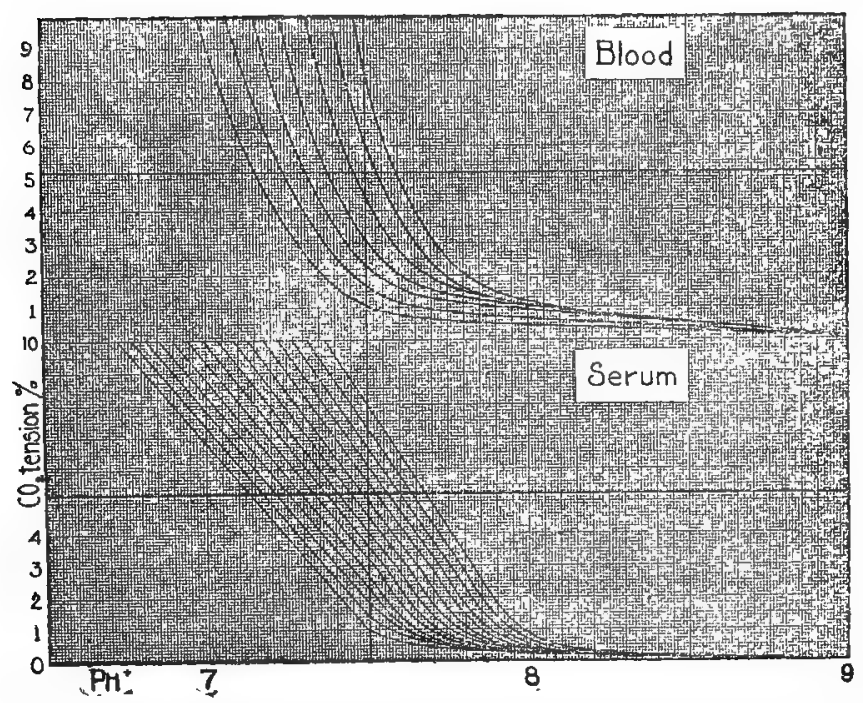

FIG. I8. Curves showing change of $\mathrm{PH}$ with change of $\mathrm{CO}_{2}$ pressure. The $\mathrm{CO}_{2}$ tension is in percentage of atmosphere (from JBC).

be replaced by a side outlet with stopcock and the fluid forced into the electrode by immersion in the beaker.

By the buffer value or reserve alkalinity of the blood, we mean its resistance to change of reaction. Since the buffer value is always expressed in arbitrary units, varying with the method of determination, those values only are comparable that are obtained by the same method. The buffer value of serum, although not the same as that of blood, is approximately proportional to it, and hence may be used in place of it (see Fig. I8). Since $\mathrm{CO}_{2}$ is the principal reagent used by the organism in maintaining the reaction of the blood, it is of interest to know the change in reaction of the blood with change in $\mathrm{CO}_{2}$ pressure. This is shown in the curve in Fig. I8. It may be noticed that within the normal physiological limits of $\mathrm{CO}_{2}$ tension the change in reaction is very small (the normal curve passing through $\mathrm{PH}=7.5$ and $\mathrm{CO}_{2}=5.2$ per cent). It is therefore necessary to take two very different $\mathrm{CO}_{2}$ pressures in order to obtain a reliable buffer value. But since the $\mathrm{PH}$ of blood in the body is rarely far from 7.5 , the whole curve may be read from Fig. I5 provided merely the $\mathrm{CO}_{2}$ 
pressure of blood (or alveolar air) is known or the $\mathrm{PH}$ of blood at any known $\mathrm{CO}_{2}$ pressure is determined.

It should be noted that hydrogen electrodes are "poisoned" by $\mathrm{H}_{2} \mathrm{~S}, \mathrm{NH}_{3}, \mathrm{Cl}_{2}$ and arsene, therefore the hydrogen should be freed from arsene by passing it through potassium permanganate $+\mathrm{KOH}$ solution and through mercuric chloride solution. If $\mathrm{H}_{2}$ is produced electrolytically the $\mathrm{O}_{2}$ should be removed (Clark and Lubs, I9I6). Putrescent solutions are best studied by Rowntree's method (see indicators).

Since the potentiometer measures the emf in millivolts, this reading must be converted into the $\mathrm{PH}$ by the following formula:

$$
\mathrm{PH}=\frac{\operatorname{emf}-337}{. \mathrm{Ig} 84 \mathrm{~T}} \text {, where } \mathrm{T}=\text { absolute temperature. }
$$

Or the conversion table, Fig. 19, may be used.

The following data will assist in constructing a conversion table like Fig. 19 of wider range:

\begin{tabular}{|c|c|c|c|c|}
\hline For $\mathrm{PH}=\mathrm{o}$ & \multicolumn{4}{|c|}{ For $\mathrm{Ph}=$ Iо } \\
\hline $\begin{array}{l}\text { Emf }= \\
337 \text { from } 18^{\circ} \text { to } 32^{\circ} \\
336.9 \text { at } 33^{\circ} \\
336.8 \text { 34 } \\
336.7 \text { 35 } \\
336.6 \text { 36 } \\
336.5 \quad 37^{\circ}\end{array}$ & $\begin{array}{l}\text { Emf } \\
914.4 \\
916.4 \\
918.4 \\
920.4 \\
922.4 \\
924.3 \\
926.3 \\
928.3 \\
930.3\end{array}$ & $\begin{array}{c}\text { Temp. } \\
\text { degrees } \\
\text { I8 } \\
\text { I9 } \\
20 \\
21 \\
22 \\
23 \\
24 \\
25 \\
26\end{array}$ & $\begin{array}{l}\text { Emf } \\
932.3 \\
934.3 \\
936.2 \\
938.2 \\
940.2 \\
942.2 \\
944.1 \\
946 . \\
947.9 \\
949.7 \\
951.6\end{array}$ & $\begin{array}{l}\mathrm{t}^{\circ} \\
27 \\
28 \\
29 \\
30 \\
31 \\
32 \\
33 \\
34 \\
35 \\
36 \\
37\end{array}$ \\
\hline
\end{tabular}

For conversion tables for the saturated calomel electrode see McClendon (I9I6 b).

The formula and conversion table refer to the emf obtained with the partial pressure of hydrogen in the electrode $=760 \mathrm{~mm}$, but since this is lowered by $\mathrm{N}_{2}, \mathrm{CO}_{2}$, water vapor, and by the barometric deficiency $(760$ - bar.) the potentiometer reading must be increased by $\left(30 \log \frac{760}{q}\right.$ ) where $q=$ partial pressure of hydrogen in the electrode. According to Clark and Lubs (I9I6) a correction should also be applied for the expansion of $\mathrm{H}_{2}$ by heat. This would make the number 338 apply to a wide 


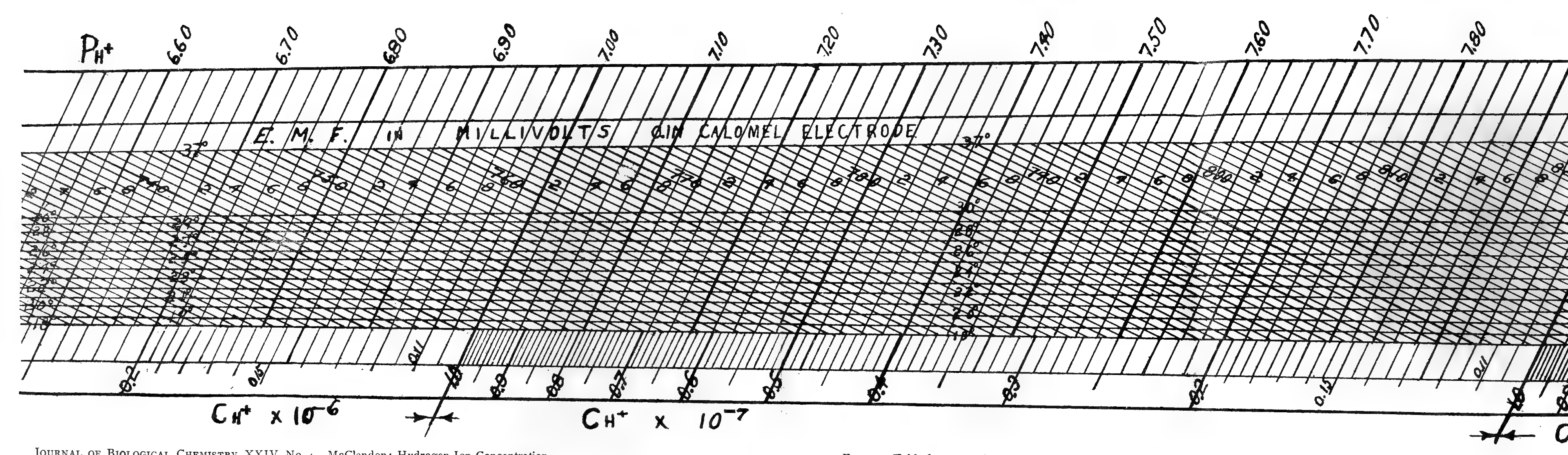




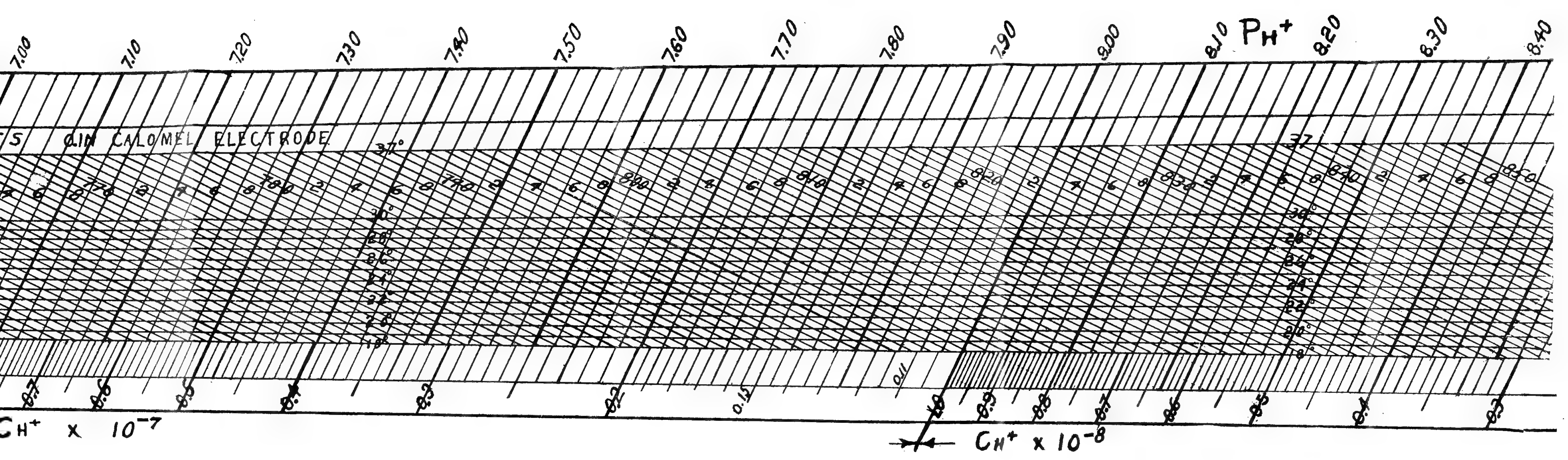

FIG. 19. Table for converting millivolts into $\mathrm{P}_{\mathrm{H}}$ and $\mathrm{C}_{\mathrm{H}}$, using o.r $\mathrm{N} \mathrm{KCl}$ calomel electrode. 
range of temperatures. All these corrections are combined in the following table from Clark and Lubs:

Correction in millivolts to be added to potentiometer reading, for change in concentration of hydrogen idue to barometric pressure, temperature and vapor tension of water.

Barometer (mm)
780
770
760
750
740

$\begin{array}{lll}20^{\circ} & 25^{\circ} & 30^{\circ} \\ 0.85 & \mathrm{I} .19 & \mathrm{I} .57 \\ \mathrm{I} .02 & \mathrm{I} .36 & \mathrm{I} .74 \\ \mathrm{I} . \mathrm{I9} & 1.53 & \mathrm{I} .92 \\ \mathrm{I} .36 & \mathrm{I} .7 \mathrm{I} & 2.10 \\ \mathrm{I} .53 & \mathrm{I} .89 & 2.28\end{array}$

Determination of Hydrogen Ion Concentration by Means of Indicators

According to Ostwald, indicators are weak acids or bases whose ions are not colored like the undissociated molecules. Indicators may, however, be used in titration in non-aqueous solvents in which dissociation is very slight. The theory of Stieglitz that the change in color is due to intramolecular change, following change in ionization, may account for the fact that the change in color may not be instantaneous. It is possible that the amount of hydration of the ions has an effect on their color, since the presence of neutral salts affects the color of indicator solutions.

The following indicators and $\mathrm{PH}$ at which they change are given by Friedenthal:

\begin{tabular}{|c|c|c|c|}
\hline Indicator & $\mathrm{PH}$ & Indicator & $\mathrm{PH}$ \\
\hline Alizarin & $6-8,11-15$ & Lacmoid .. & $4-8$ \\
\hline Alizarin blue $S$. & II-I $3,7-8$ & Magdalarot 3-4, I +-15. . & $3-4,14-15$ \\
\hline $\begin{array}{l}\text { Alizarin green } \mathrm{B} . \ldots . . \\
\text { Alizarin } \mathrm{Na} \text { sulfonate. }\end{array}$ & I2-13 & Mauvein $\ldots . . . \ldots \ldots \ldots$ & $0-3,13-15$ \\
\hline 11kali green ......... & $\begin{array}{l}4-0 \\
3-7\end{array}$ & Methyl orange ........... & $2-5,14-15$ \\
\hline ilmin (litmus) & $5-9$ & Methyl violet. & $0-3, \pm 2-15$ \\
\hline opurpurin B. & $0-5,13-14$ & a-Napthol benzoir & 9-II \\
\hline eralmondoilgreen.. & $0-2$, I I-I 4 & Nentral red & $0-1,7-9$ \\
\hline Cochineal......... & 4- 6 & p-Nitrophenol & \\
\hline Congo red & $3-5$ & Phenacetolin & $3-6,10-13$ \\
\hline & I2-I4 & Phenolphthalein & 8-10, I3-I5 \\
\hline Curcumein (Tumeric). & I3-15 & Rosolic acid & $6-8$, I3-I5 \\
\hline lin ... & $6-8$ & Safranin & $0-1, I 4-15$ \\
\hline Dimethylamidoazobenzo & $12-4$ & Saurefuchsin (Ac & \\
\hline Echtrot & $0-i$ & fuchsin) $\ldots . .$. & 9-I4 \\
\hline eneblue & $0-3$, & Tetrabromphenolphtha- & \\
\hline Fluorescein & $0-6$ & lein & $8-9, \mathrm{I} 2-\mathrm{I} 5$ \\
\hline Gallein ... & I- 6 , & Thymolphthalein & IO-II \\
\hline & $7-8$ & Tropaeolin & $7-9$ \\
\hline & $0-15$ & $\operatorname{Tr}$ & II-I3 \\
\hline & II-I3 & $\operatorname{lin} 0$ & $0-3$ \\
\hline & I $2-14$ & $\operatorname{lin} \infty$ & II-13 \\
\hline $\sin 2-$ & $0-15$ & Trinitro benzol & I $2-15$ \\
\hline
\end{tabular}


The following indicators were found by Sörensen (Igog) to be least affected by proteins, but some control is necessary if the protein content of the unknown solution is considerable.

Indicator

$\mathrm{PH}$

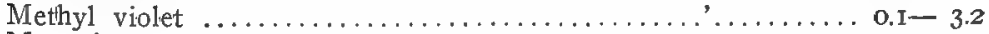

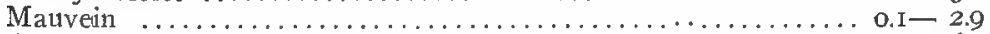

(Methyl red) o-Benzolcarbonicacid azo dimethyl anilin..... 4.2-6.3

p-Nitro phenol $\ldots \ldots \ldots \ldots \ldots \ldots \ldots \ldots \ldots \ldots \ldots \ldots \ldots \ldots \ldots \ldots, 5-7$

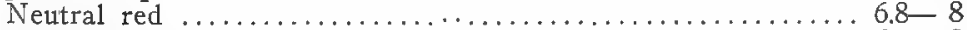

Rosolic acid ..............................6.9-8

(Tropaeolin ooo) p-Benzolsulfonicacidazo-a-11apthol........ 7.6- 8.9

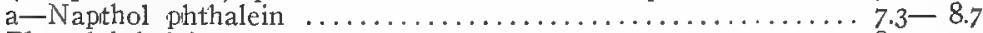

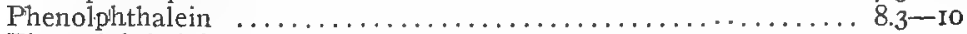

Thymolphthalein $\ldots \ldots \ldots \ldots \ldots \ldots \ldots \ldots \ldots \ldots \ldots \ldots \ldots .6 .3$ - 10.5

The following indicators were sludied by Clark and Lubs (1915):

Indicator

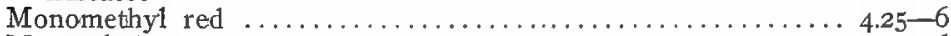

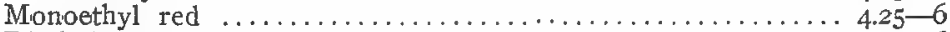

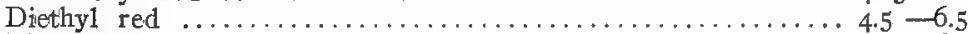

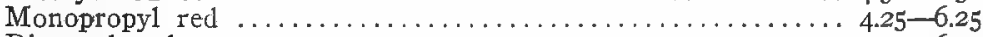

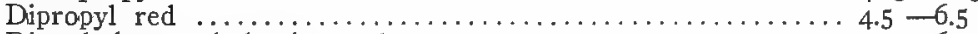

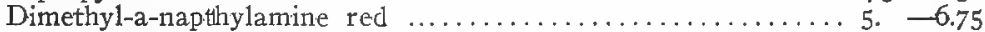

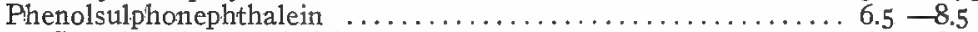

o-Cresolsulphonephthalein $\ldots \ldots \ldots \ldots \ldots \ldots \ldots \ldots \ldots \ldots .6 \ldots \ldots .6 .5$

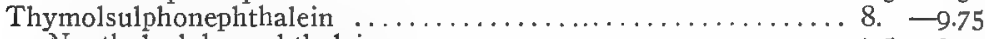

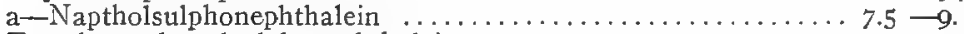

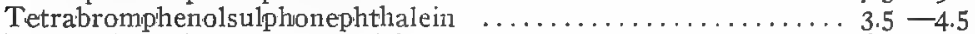

Dibromthymolsulphonephthalein ............... -7.25

The color change and $\mathrm{PH}$ of solutions of some useful indicators were shown in a colored chart. In order to find the $\mathrm{PH}$ of an unknown solution, pour it into a test tube of $\mathrm{I} \mathrm{cm}$ bore, add o.I cc of the indicator solution, and while looking down into it against a white background, compare it with the chart. If the test tube is $2 \mathrm{~cm}$ bore, $.4 \mathrm{cc}$ of indicator solution should be used. (McClendon, I9I6, b; also in Tortugas Vol., Carnegie Ins. Wash., in press.)

As shown by Sörensen (Ig09) some indicators are more sensitive than others to neutral salts, proteins and protein decomposition products. Congo red and litmus are therefore to be especially shunned in biological work. For solutions containing large amounts of proteins, or having a color of their own, the method of Rowntree, Marriott and Levy, I9I5, is useful, provided that obvious extra precautions are recognized. The fluid to be tested is placed in a dialyzing capsule of collodion or parchment paper impermeable to protein, and introduced into a test tube containing some neutral (boiled) distilled water or salt solution. The test tube is stoppered to prevent the loss of $\mathrm{CO}_{2}$ and 
dialysis allowed to proceed to approximate equilibrium. The capsule is removed and the right quantity of indicator added. The color is compared with that of a series of tubes of the same bore, containing the same amount of indicator and solution but of known PH. (Such tubes may be obtained, sealed, from Hynson, Westcott and Co., Baltimore.) Or the tube may be compared with the colored chart. One effect of dialysis is to dilute the solutes affecting the $\mathrm{PH}$ and causing an error which is less the greater the buffer value of the solution.

\section{Buffers and Solutions of Standard Hydrogen Ion Concentration}

The $\mathrm{PH}$ of water, or solutions of neutral salts, or very dilute solutions of strong acids or alkalis, is changed by minute traces of certain impurities. Absolutely pure water (conductivity water) is neutral but may not remain so. If exposed to the air it becomes acid due to absorption of $\mathrm{CO}_{2}$, and if kept in glass it becomes alkaline, due to solution of the glass. "Pyrex" or "nonsol" glass is better in this respect, but any glass is improved by allowing a jet of steam to condense on the surface and drain off, a process known as steaming out. Silica or metal vessels are more reliable. This difficulty in maintaining fixed hydrogen ion concentrations is obviated by the use of buffers. The chief buffer in organisms is $\mathrm{NaHCO}_{3}$. The reaction of a pure solution of $\mathrm{NaHCO}_{3}$ is but very slightly alkaline, that of $\mathrm{Na}_{2} \mathrm{CO}_{3}$ somewhat more alkaline and that of $\mathrm{CO}_{2}$ but slightly acid. If we add a strong acid to a solution of $\mathrm{NaHCO}_{3}$ the acidity of the mixture will not exceed that of a solution of $\mathrm{CO}_{2}$ until all of the $\mathrm{NaHCO}_{3}$ has been decomposed. If we add a strong alkali to a solution of $\mathrm{NaHCO}_{3}$ the alkalinity of the solution will not exceed that of a $\mathrm{Na}_{2} \mathrm{CO}_{3}$ solution until all of the $\mathrm{NaHCO}_{3}$ has been decomposed. One drop therefore of $\mathrm{n} \mathrm{HCl}$ would acidify a liter of $\mathrm{H}_{2} \mathrm{O}$ or neutral salt solution more than a liter of the acid would acidify a liter of $\mathrm{m} \mathrm{NaHCO}$ solution-hence the advantage of a relatively high concentration of buffers in standard solutions. The standard solutions used by Sörensen (I9I2) are shown in the chart in Fig. 20. On the ordinate is given the number of $\mathrm{cc}$ of the first solution of a pair to be taken, when the second solution is to be added to give a total volume of Io cc. The stock solutions are as follows: 
"HCl" = A O.I n solution of $\mathrm{HCl}$ titrated with silver nitrate.

" $\mathrm{NaOH}$ " $=$ A o.I $\mathrm{n}$ solution of $\mathrm{NaOH}$ made by adding an excess of sodium or sodium amalgam to $\mathrm{CO}_{2}$-free $\mathrm{H}_{2} \mathrm{O}$ and titrating with $\mathrm{HCl}$ and diluting to. $\mathrm{n}$. Or decanting a clear saturated solution of $\mathrm{NaOH}$ and diluting to required volume with $\mathrm{CO}_{2}$-free $\mathrm{H}_{2} \mathrm{O}$.

"Citrate" = A o.I $\mathrm{m}$ solution of secondary sodium citrate made by dissolving $2 \mathrm{I} .008 \mathrm{~g}$ citric acid in $800 \mathrm{cc}$ of $.25 \mathrm{n} \mathrm{NaOH}$ and diluting to a liter. A layer of ether on top will preserve it.

" $\mathrm{H}_{3} \mathrm{PO}_{4}$ " $\mathrm{A}$ I/ $\mathrm{I} 5 \mathrm{~m}$ solution of $\mathrm{H}_{3} \mathrm{PO}_{4}$ accurately standardized.

" $\mathrm{KH}_{2} \mathrm{PO}_{4}$ " $=\mathrm{A}$ I/15 $\mathrm{m}$ solution of $\mathrm{KH}_{2} \mathrm{PO}_{4}$ made by dissolving $9.078 \mathrm{~g}$ of the recrystallized anhydrous (desiccated) salt to the liter.

" $\mathrm{Na}_{2} \mathrm{HPO}_{4}$ " = $\mathrm{A} \mathrm{m/I} 5$ solution of $\mathrm{Na}_{2} \mathrm{HPO}_{4}$ made by dissolving Ir.876 $\mathrm{g}$ to the liter, of $\mathrm{Na}_{2} \mathrm{HPO}_{4} 2 \mathrm{H}_{2} \mathrm{O}$ that has been recrystallized and dried until it has the right water content by analysis.

"Borate" = A solution of $\mathrm{I} 2.404 \mathrm{~g}$ of recrystallized desiccated boric acid + IOO cc n NaOH solution made up to I liter.

The distilled water used in making these solutions should be free from $\mathrm{NH}_{3}$ and $\mathrm{CO}_{2}$. The $\mathrm{CO}_{2}$ may be practically eliminated by boiling I 5 minutes in a tin vessel and cooling under a soda lime tube. All solutions should be kept in "nonsol" or "pyrex" glass vessels or in paraffined bottles and those with $\mathrm{PH}$ above 5 should be provided with soda lime tubes. The reagents should be the purest. In dropping metallic sodium into water $\mathrm{O}_{2}$ should be excluded. $\mathrm{NaOH}$ made from $\mathrm{Na}$ may be purchased but should be freed from carbonate by making it first an 80 per cent solution in a stoppered bottle and allowing the carbonate to precipitate, then pipetting off the clear solution, diluting with $\mathrm{CO}_{2}$ free water and standardizing it. All of the salts should be dissolved in boiling $\mathrm{H}_{2} \mathrm{O}$, filtered and crystallized at least once. It is difficult to obtain pure phosphates. The $\mathrm{Na}_{2} \mathrm{HPO}_{4}$ solution should turn phenolphthalein a deep red. In the chart, Fig. 20, impurities in the reagents affect the ends of the curves more than the middle.

The buffer mixtures of Clark and Lubs (I9I6) are as follows: 




FIG. 20. Sörensen's chart of buffer mixtures. On the ordinate is the number of $\mathrm{cc}$ of the first solution when the mixture is completed to to $\mathrm{cc}$ with the second solution indicated on the curve. On the abscissa is the $\mathrm{PH}$ at $18^{\circ}$ (from EP). 
I. Solutions made up to $200 \mathrm{cc}$ and containing $50 \mathrm{cc} 0.2 \mathrm{~m} \mathrm{KH}$-phthalate +

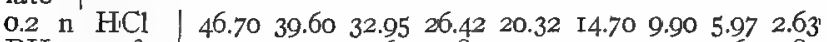

\begin{tabular}{l|lllllllll}
$\mathrm{PH}$ at $20^{\circ}$ & 2.2 & 2.4 & 2.6 & 2.8 & 3.0 & 3.2 & 3.4 & 3.6 & 3.8
\end{tabular}

2. Solutions made up to $200 \mathrm{cc}$ and containing $50 \mathrm{cc} 0.2 \mathrm{~m} \mathrm{KH}-\mathrm{phtha-}$ late +

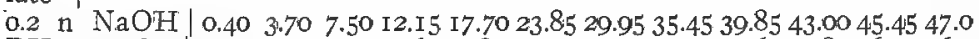
\begin{tabular}{ll|lllllllllllll}
$\mathrm{PH}$ & at $20^{\circ}$ & 4.0 & 4.2 & 4.4 & 4.6 & 4.8 & 5.0 & 5.2 & 5.4 & 5.6 & 5.8 & 6.0 & 6.2
\end{tabular}

3. Solutions made up to $200 \mathrm{cc}$ and containing $50 \mathrm{cc} 0.2 \mathrm{~m} \mathrm{KH}_{2} \mathrm{PO}_{4}+$ $50 \mathrm{cc} 0.2 \mathrm{n} \mathrm{KCI}+$

$\begin{array}{lllllllllllll}\mathrm{PH} \text { at } 20^{\circ} & 5.8 & 6.0 & 6.2 & 6.4 & 6.6 & 6.8 & 7.0 & 7.2 & 7.4 & 7.6 & 7.8 & 8.0\end{array}$ $0.2 \mathrm{n} \mathrm{NaOH} \mid 3.72 \quad 5.70 \quad 8.60 \mathrm{I} 2.60$ I7.80 $23.6529 .6335 .00 \quad 39.5042 .8045 .2046 .80$

4. Solutions made up to $200 \mathrm{cc}$ and containing $50 \mathrm{cc}$ of $0.2 \mathrm{~m} \mathrm{H}_{3} \mathrm{BO}_{3}+$

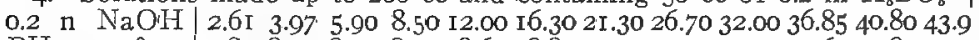

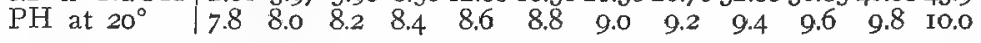

These solutions should be made from the purest reagents, that have been recrystallized three times. The acid potassium phthalate, acid potassium phosphate, and potassium chloride may be dried at $\mathrm{IIO}^{\circ}$ but the boric acid must be dried at room temperature in a desiccator.

The more acid of the mixtures will deposit crystals of phthalic acid if not kept considerably above $20^{\circ}$.

It may be inferred that the buffer value of these solutions is less than those of Sörensen, but their salt action on indicators is also less.

\section{Dissociation Constants of Acids and Bases}

Acids and bases differ from neutral salts in that among their dissociation products are the ions of water, $\mathrm{H}^{*}$ or $\mathrm{OH}^{\prime}$ as the case may be. Since the strength of acids and bases lies in the number of $\mathrm{H}$ and $\mathrm{OH}$ ions they dissociate, it is determined by the dissociation constant, $\mathrm{c}$.

From the law of mass action we have: $c=\frac{a^{2}}{v(r-a)}$, where $v$ is the number of liters in which I mol of the acid (for instance) is dissolved, $I$ - a represents the proportion of the undissociated molecules and $a$ represents the proportion of anions $=$ cations. If we increase the dilution, $v$, then $a^{2}$ increases and therefore $\left(a=\mathrm{H}^{*}\right)$ increases. ( $\mathrm{H}^{*}$ per liter decreases.)

Strong acids appear to disobey this law when dissociation is determined by electric conductivity. If $c$ is determined from the dissociation of a more concentrated solution, a more dilute solution is found to be dissociated less than is calculated from the formula. In other words, the dissociation in concentrated 
solutions is more than is expected. The anomaly may be explained by the hypothesis that the hydration of ions is less in the concentrated solution, they move faster and increase the conductivity, and, hence, the high dissociation is only apparent (G. N. Lewis). The dissociation of concentrated solutions of $\mathrm{HCl}$ as calculated from electrode potential (J. Ellis, 1916) is much greater than that calculated from conductivity data. The two curves for dilute solutions are shown in Fig. 2I.

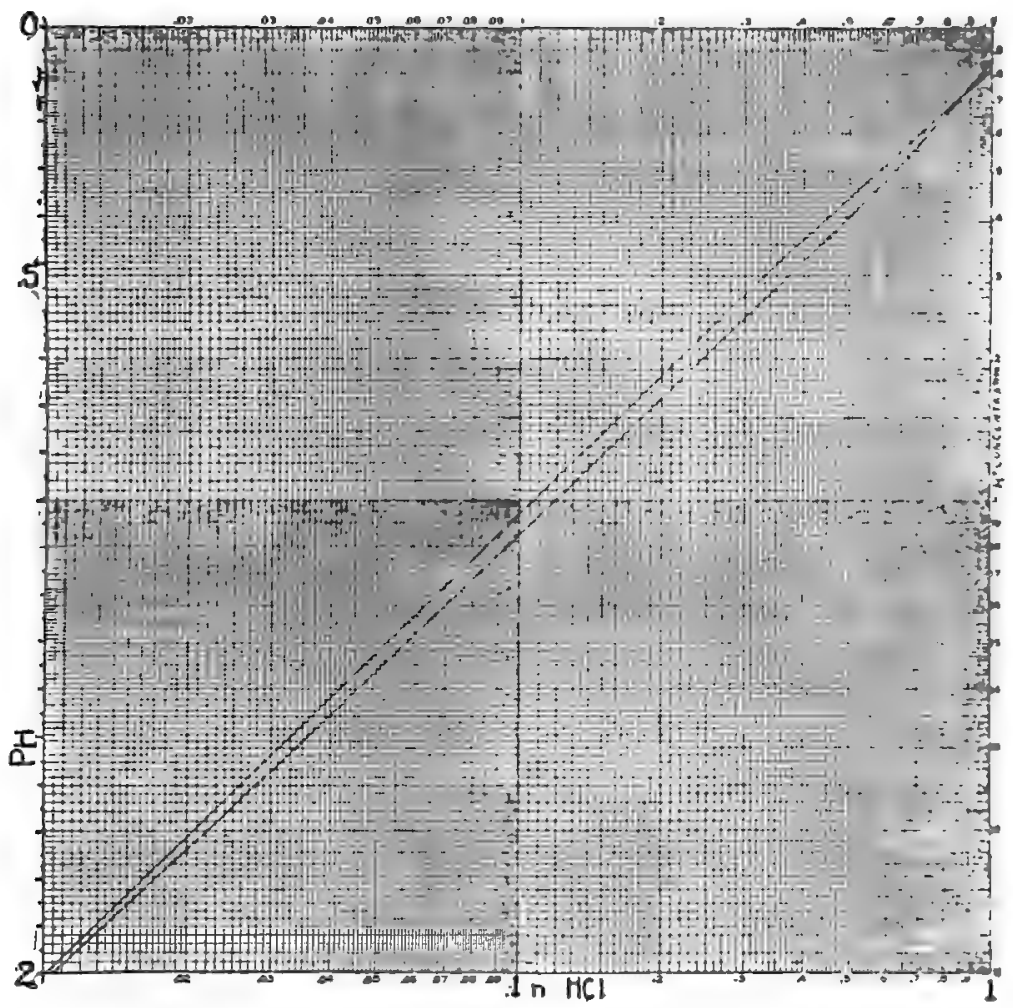

FIG. 2I. Curves showing the difference between the dissociation of $\mathrm{HCl}$ as measured by electric conductivity (conductance ratio) and electrode potential (hydrogen ion concentration).

In a molecular solution of an acid $z^{\prime}=I$ and the formula becomes: $c=\frac{a^{2}}{a-I}=\frac{H^{*} \times A^{\prime}}{H A}$, where $H^{*}$ and $A^{\prime}$ represent 
the concentration of the ions and HA of the undissociated molecules of the acid.

The dissociation constants of some weak acids and substances which dissociate traces of $\mathrm{H}^{\circ}$, are as follows at $25^{\circ}$ (mostly from Scudder, I9I 5) :

\begin{tabular}{|c|c|c|c|}
\hline$-\log K_{A}$ & Acid & $-\log K_{A}$ & Acid \\
\hline$-.92^{21}$ & Trichloracetic & I 0.22 & Carbonic $\left(\mathrm{CO}_{3}{ }^{\prime \prime}\right)$ \\
\hline+1.00 & Oxalic & II 1.00 & Chloral hydrate \\
\hline I. 29 & Dichloracetic & 11.74 & Maltose \\
\hline I.96 & Phosphoric $\left(\mathrm{H}_{2} \mathrm{PO}_{4}{ }^{\prime}\right)$ & I 2.06 & Levulose \\
\hline $2.8 \mathrm{I}$ & Monochloracetic & $\mathrm{I} 2.2 .3$ & Dextrose \\
\hline 2.96 & Salicylic & $\mathrm{I} 2.23$ & Lactose \\
\hline 3.01 & Tartaric & 12.43 & Arabinose \\
\hline 3.08 & CitrIc & $12 .+4$ & Phosphoric $\left(\mathrm{PO}_{4}^{\prime \prime \prime}\right)$ \\
\hline 3.40 & Malic & 12.72 & Sucrose \\
\hline 3.65 & Hippuric & 13.47 & Mannite \\
\hline 3.68 & Formic & 14.15 & Glycerol \\
\hline 3.82 & Acetoacetic & 14.74 & Propionitrile \\
\hline 3.85 & Lactic & $\log \mathrm{K}_{\mathrm{D}}$ & Base \\
\hline 4.I7 & Succinic & $\log ^{11} \mathrm{~B}$ & Pineridine \\
\hline 4.18 & Benzoic & 2.80 & Piperidine \\
\hline 4.25 & Acrylic & 2.90 & Diethylamine \\
\hline 4.44 & Adipic & 3.25 & Ethylamine \\
\hline 4.49 & Gallic & 3.27 & Dimethylamine \\
\hline 4.7 & Betaoxybutyric & 3.39 & Methylamine \\
\hline 4.73 & 'Acetic & 4.07 & Ethylenediamine \\
\hline 4.80 & Buitynic & 4.22 & Trimethylamine \\
\hline 4.82 & Valerianic & 4.34 & Allylamine \\
\hline 4.84 & Propionic & 4.74 & Ammonia \\
\hline 4.84 & Caproic & 4.77 & Brucine \\
\hline 4.84 & Caprylic & 5.58 & Quinine \\
\hline 5.80 & Uric & 6.07 & Strychnine \\
\hline 5.80 & Salicyl aldehyde & 7.00 & Atropine \\
\hline 6.00 & Tannic & 7.00 & Pilocarpine \\
\hline 6.05 & Phosphoric $\left(\mathrm{HPO}_{4}{ }^{\prime \prime}\right)$ & 8.64 & Pyridine \\
\hline 6.19 & Cacodylic & 9.30 & Aniline \\
\hline 6.52 & Carbonic $\left(\mathrm{HCO}_{3}{ }^{\prime}\right)$ & 9.50 & o-Toluidine \\
\hline 7.24 & Hydrogen sulphide & 9.58 & Methylaniline \\
\hline 9.24 & Boric $\left(\mathrm{H}_{2} \mathrm{PO}_{3}{ }^{\prime}\right)$ & 9.62 & Dimethylaniline \\
\hline 9.33 & Hydrocyanic & 9.80 & Ethylaniline \\
\hline 9.92 & Phenol & I 3.80 & Urea \\
\hline
\end{tabular}

The temperature coefficient for the dissociation of acids and bases is usually small.

In case of $\mathrm{CO}_{2}$ and $\mathrm{NH}_{3}$, the above figures show only the apparent dissociation constants. The real acid and base are $\mathrm{H}_{2} \mathrm{CO}_{3}$ and $\mathrm{NH}_{4} \mathrm{OH}$, but the concentration of these is unknown. According to the investigations of Thiel and Strohecker (IgI4) the dissociation constant of $\mathrm{H}_{2} \mathrm{CO}_{3}$ is greater than that of formic acid. 


\section{Dissociation of Amphoteric Electrolytes (Ampholytes)}

Ampholytes dissociate both $\mathrm{H}$ and $\mathrm{OH}$ ions, having therefore two dissociation constants, $\mathrm{K}_{\mathrm{A}}$ and $\mathrm{K}_{\mathrm{B}}$. A pure solution of an ampholyte has an acid reaction, if $\mathrm{K}_{\mathrm{A}}$ is the greater, and an alkaline reaction if $\mathrm{K}_{\mathrm{B}}$ is the greater. The following table gives the dissociation of some amino acids and dipeptides at $25^{\circ}$. According to these figures, mostly from Scudder (I9I4), the dipeptides are dissociated to a greater extent than the amino acids from which they are formed, and the dissociation of $\mathrm{H}^{*}$ has increased more than the dissociation of $\mathrm{OH}^{\prime}$, making the dipeptides more strongly acid than the amino acids.

\begin{tabular}{|c|c|c|c|}
\hline$-\log \mathrm{K}_{\mathrm{A}}$ & Ampholyte & $-\log K_{B}$ & $\begin{array}{l}\text { PH of Iso- } \\
\text { electric Point }\end{array}$ \\
\hline $3.85^{\Omega}$ & Aspartic acid & I 1.92 & 3.03 \\
\hline 4.39 & Glutamic acid & $\ldots$. & $\ldots \ldots$ \\
\hline 4.79 & m-Aminobenzoic acid & IO.gI & 3.79 \\
\hline 4.92 & p-Aminobenzoic acid & II .63 & 4.20 \\
\hline 7.74 & Glycylglycine & 10.70 & 5.52 \\
\hline 7.74 & Alanylglycine & 10.70 & 5.52 \\
\hline 7.82 & Leucylglycine & 10.52 & 5.66 \\
\hline 8.16 & Taurine & .... & ..... \\
\hline 8.28 & Asparigine & II.74 & 5.27 \\
\hline 8.40 & Tyrosine & II. 58 & $5.4 I$ \\
\hline 8.66 & Phenylalanine & I I 89 & 5.35 \\
\hline 8.66 & Histidine & 8.24 & $7.2 I$ \\
\hline 9.70 & Alanine & I I. 28 & 6.72 \\
\hline 9.74 & Glycine & II. $55^{\circ}$ & 6.58 \\
\hline 9.74 & Leucine & I I. 49 & 6.54 \\
\hline I 1.00 & Lysine & 7.00 & 9.00 \\
\hline I3.96 & Arginine & 7.00 & 10.48 \\
\hline
\end{tabular}

These dissociation constants show the relative dissociation of $\mathrm{H}$ and $\mathrm{OH}$ ions by an ampholyte when dissolved in a neutral solute. But the addition of an acid to the solution will reduce the dissociation of $\mathrm{H}$ ions and the addition of a base will reduce its dissociation of $\mathrm{OH}$ ions by the ampholyte, according to the law of mass action. At a certain reaction of the solution for each ampholyte, the dissociation of $\mathrm{H}$ and $\mathrm{OH}$ ions will be equal. The "remainder" of the molecule will be therefore neither electronegative nor positive, but neutral or isoelectric. The reaction at which this occurs is called the isoelectric point. According to Michaelis the $\mathrm{PH}$ of the isoelectric point is one half of $\left(-\log \mathrm{K}_{\mathrm{W}}\right)+\left(-\log \mathrm{K}_{\mathrm{A}}\right)-\left(-\log \mathrm{K}_{\mathrm{B}}\right)$ and the $\mathrm{PH}$ so calculated agrees with the $\mathrm{PH}$ found by experiment. Isoelectric points are given in above table, those of proteins in Chapter VI. 


\section{CHAPTER V}

\section{SURFACE TENSION AND ADSORPTION}

We are sometimes surprised at the strength of the surface film when we see insects supported on the surface of water, but according to Colton (I9Io) a small clam suspended itself by threads (byssus) attached at only three points from the surface film in the Naples aquarium. This is more surprising when we realize that the thickness of the film is $.00000006 \mathrm{~mm}$.

Although instances were already known, it was first formulated by J. Willard Gibbs ( 1874 ) into a general rule that substances which lower the surface tension of a solvent become more concentrated in the surface film than in the interior. Take for example a system of two phases, oil and water. If a substance which lowers the surface tension of water be dissolved in the water it will concentrate in the surface film, next to the oil. If the substance be soluble in oil, it will diffuse into the oil until an equilibrium is reached between the concentration of the substance in the oil and in the surface film of the water. If this substance therefore has the same solubility in water and in oil and does not lower the surface tension of oil it will become more concentrated in the oil than in the water. If the substance be insoluble in the oil it will remain concentrated in the film of water next to the oil and is said to be adsorbed by the oil. Hence, surface tension, adsorption and diffusion are related phenomena.

In the phase boundary between two fluids it is easier to measure the surface tension than the adsorption. On the contrary it is impossible to measure the surface tension in the phase boundary between a fluid and a solid, but the adsorption may be measured, actually or relatively.

The most practicable method of measuring surface tension, in biochemistry, is the estimation of the average weight of a drop of the one of the fluids falling from a standardized pipette into the other fluid. In most measurements one of the fluids 

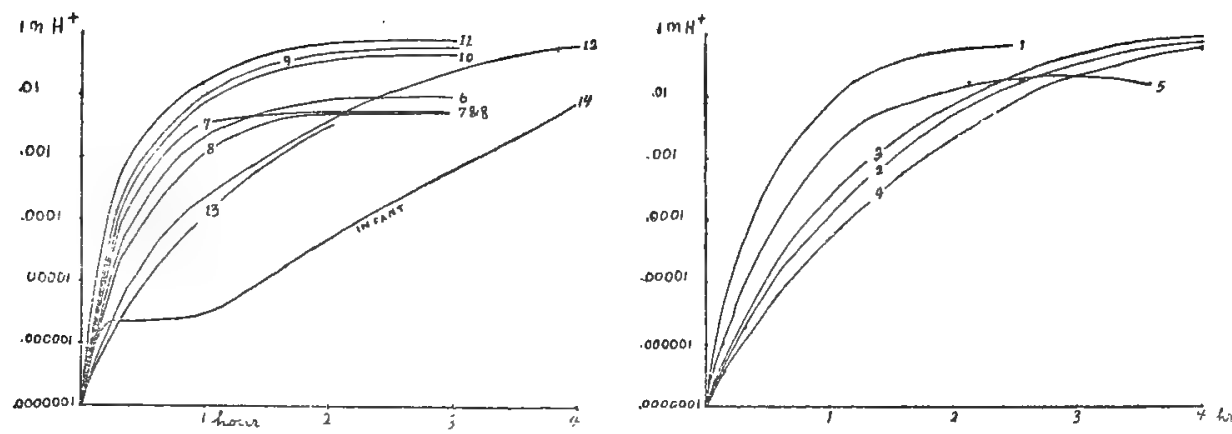

Charts showing the hydrogen ion concentration of the stomach (on the ordinate) and time after eating (on the abscissa). 'Curves I-4 from same man with different quantities of food. Curve 14 is the average curve for infants.

is air, and the drop is hence allowed to drop out of the pipette into the air. The diameter of the dropping surface $=4.6$ to 5.7 $\mathrm{mm}$ (J. L. Morgan, I9I5). One form of pipette is Traube's (I904) stalagmometer. In its use, the weight of the drop is found by multiplying the volume of the drop by its specific gravity, the volume of the drop found by dividing the volume of the pipette by the number of drops from one filling. The pipette is standardized with water, whose surface tension is taken as unity. Therefore the surface tension $=$

specific gravity of solution $X$ no. of drops of water

number of drops of solution

Traube's (I9I2) viscostagmeter is a dropping burette with which the volume of say ten drops is measured, and is more rapid than the stalagmometer, which holds forty to roo drops of water. (Stalagmometers are made to order by Kimball Durand Co., Chicago.)

It is the presence of the substance in the surface film which lowers the surface tension, but it is not until diffusion into the surface film has reached an equilibrium that the lowest surface tension is present. For instance, soap very greatly lowers the surface tension of water, but when measurements are taken on new surfaces by the instantaneous method of Rayleigh, the surface tension of the soap solution is found to be the same as 
that of water. If the soap solution is allowed to drop slowly from a stalagmometer it shows a lower' surface tension than if dropped fast. The rate of flow from a stalagmometer is reduced by a piece of capillary tubing forming part of the outflow tube, but the more viscous the fluid the slower it will flow. In order to reduce errors from this source, Traube uses three stalagmometers for solutions of different viscosity and selects one delivering less than one drop per second. The error in using the stalagmometer, due to lack of diffusion equilibrium, is less than the error in the methods used on old surfaces, due to the formation of haptogen membranes. It should be remembered, however, that the stalagmometric data are merely comparative, and only those results should be compared where the flow is slow and its rate approximately the same in each case (see Harkins and Humphrey, I916).

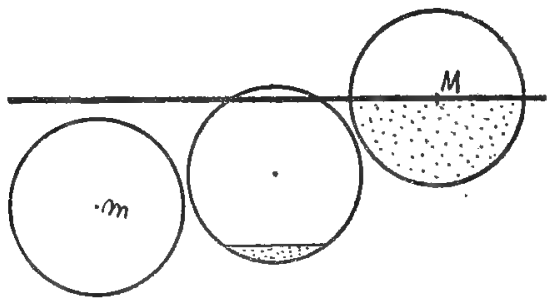

FIG. 22. Scheme showing that surface tension is located in a film whose thickness equals the diameter of the sphere of molecular attraction.

In order to understand the relation between surface tension and osmotic pressure certain theoretical considerations are necessary. Surface tension is a molecular phenomenon. The thickness of the surface film equals the radius of the sphere of molecular attraction. In Fig. 22, suppose $m$ to represent a molecule in the interior, $M$ a molecule on the surface of a liquid, and the circles around them to define the spheres of molecular attraction. The sphere of $m$ is entirely within the liquid, hence $m$ attracts and is attracted equally on all sides by all molecules in this sphere. On the contrary, only the hemisphere of $M$ is within the liquid and, hence, $M$ is attracted laterally and downward, but not upward. This attraction which the molecules in the surface film have for one another puts the film under a tension-surface tension. 
If the surface be convex the surface tension is greater, increasing with the convexity. In Fig. $23, m$ and $M$ are two molecules equidistant beneath a plane surface $(m)$ and a convex surface $(M)$. These molecules attract and are attracted by the

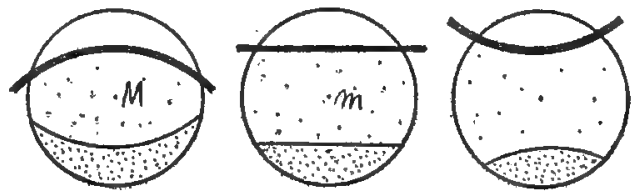

FIG. 23. Scheme showing that convexity increases surface tension and concavity decreases it.

molecules within the lightly shaded areas, the spheres of molecular attraction. The uncompensated downward attractions are represented by the more deeply shaded areas. The area is larger in the convex film and, hence, the surface tension greater.

The interior of a drop of a liquid is under a hydrostatic pressure due to its surface tension, the pressure being greater the smaller the drop. Imagine a spherical drop bisected by a plane. If the surface tension is unity, the pull of the surface film on the plane $=2^{\pi_{r}}=$ the hydrostatic pressure on one side of the plane $=\mathrm{p}^{\pi \mathrm{r}^{2}}$, where $p$ is the pressure. Since $\mathrm{p}^{\pi} \mathrm{r}^{2}=2^{\pi} \mathrm{r}$, $\mathrm{p}=\frac{2^{\pi_{1}}}{\pi_{\mathrm{r}^{2}}}=\frac{2}{\mathrm{r}}$, hence when $r$ is small $p$ is large, or when the radius is small the pressure is large. Thus, the pressure is greater in small drops when the surface tension remains the same. But it was shown above that the surface tension is greater the smaller the drop (the greater the curvature). Hence, the pressure is increased in two ways.

Now suppose the surface film of a drop of liquid that floats in another liquid of equal osmotic pressure to be semipermeable. The surface tension of the drop causes a hydrostatic pressure in its interior and pure water will be squeezed out through the surface film until the increase in osmotic pressure equals and replaces the hydrostatic pressure due to surface tension. Hence surface tension increases osmotic pressure.

Without knowing the surface tension of protoplasm we cannot estimate the effect of surface tension on the osmotic pressure 
of cells. Quincke assumed that the surface tension of protoplasm is the difference between the surface tension of water and that of a certain albumin solution. From this assumption the osmotic pressure of Micrococcus progrediens (diameter $=.16 \mathrm{~mm}$ ) would be 5.75 atmospheres greater than the medium, without taking into account the increase in surface tension due to convexity. This effect decreases rapidly with the diameter of the cell, for one of $.02 \mathrm{~mm}$ diameter being only .046 atmospheres.

\section{Surface Tension of Aqueous Solutions}

As a rule, inorganic neutral salts, and many sugars, very slightly raise the surface tension, whereas acids, bases and most organic substances lower the surface tension. The lowering is not proportional to the concentration, but when a small amount of the substance lowers the tension a certain degree, each addition of the same amount has less and less effect, Fig. 24.

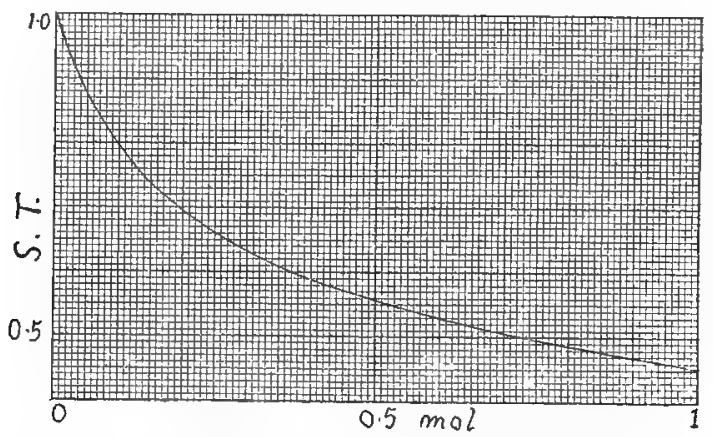

FIG. 24. Surface tension-adsorption curve (adsorption isotherm) of butyric acid (from a class experiment). The surface tension relative to pure water is plotted on the ordinates and the mol concentration of the butyric acid on the abscissae.

A curve similar to Fig. 24, which is the typical surface tension adsorption or adsorption isotherm curve was first figured by Dupré (1866). The necessary consequence of the shape of this curve is that the solute reducing the surface tension is adsorbed. This relation, demonstrated by Dupré and formulated a few years later by Gibbs, was first called to the attention of physiologists through the comparatively recent writings of Wilhelm Ostwald. 
The following table gives the surface tension at $15^{\circ}$ of .25 molecular solutions of some non-electrolytes, according to Traube. It may be seen that sugars and amino acids raise the surface tension, urea is inactive, trihydric alcohols and amides lower it slightly, and monohydric alcohols, ketones, nitrils, and organic acids and their esters lower it very much. In homologous series the effect is greater the longer the carbon chain. According to Traube it requires only one-third the molecular concentration of one member of the series to lower the tension as much as the preceding member.

\begin{tabular}{|c|c|c|}
\hline \multicolumn{3}{|c|}{ s.t. at $15^{\circ}$} \\
\hline $\begin{array}{l}\text { Cane sugar } \\
\text { Grape sugar }\end{array}$ & ....1.007 & Propionic acid \\
\hline $\begin{array}{l}\text { Grape stuga } \\
\text { Mannite }\end{array}$ & ...I.004 & $\begin{array}{l}\text { Methyl acetate } \\
\text { Propyl alcohol }\end{array}$ \\
\hline Glycocoll & $\ldots \ldots$ ז.003 & Methyl ethyl ketone... \\
\hline Water .. & $\ldots I$ & Diethylamine .. \\
\hline Urea & & Piperidine. \\
\hline Glycerine & . . .998 & Ethyl ether \\
\hline Glycol & & Pyridine \\
\hline Acetamid & 986 & Paraldehyde \\
\hline Methyl alcoh & .966 & Chloral hydrate \\
\hline tic acid & .961 & Butyric acid \\
\hline Aceto nitrile & .95 & Isobutyl alcohol \\
\hline $\begin{array}{l}\text { Ethyl aloohol } \\
\text { Acete }\end{array}$ & $\cdots \cdot .92$ & Tert. Amyl alcohol. \\
\hline Ethyl uretha & .80 & Ethyl acetate....... \\
\hline ropionitrile. & $\ldots .84$ & \\
\hline
\end{tabular}

Although small amounts of some substances may reduce the surface tension of water to less than half, no substance raises it very much. The explanation is that the substance which lowers the surface tension becomes very concentrated in the surface film and has its maximal effect, whereas the substance which raises the surface tension is driven from the surface, consequently reducing its effect.

The salts of mineral acids and alkalis slightly raise the surface tension whereas $\mathrm{HCl}$ and $\mathrm{HNO}_{3}$ lower it. An electrolyte brings with it the added effects of the ions and molecules.

Surface tension is reduced by the colloids from organisms, but since we do not know their molecular weight we cannot compare them quantitatively with other organic substances. The colloid concentrated in the surface film becomes very viscous, finally forming a membrane insoluble in water. These so-called haptogen membranes form on the surface between water and air, ether, carbon bisulphide, chloroform and many solids. By 
shaking the solution the colloid may be precipitated in the form of torn membranes, which assume a stringy appearance. In this way enzymes may be inactivated, being either colloidal or precipitated with colloid impurities. The presence of colloids makes an emulsion of oil and water permanent by forming haptogen membranes around the oil drops. It is the haptogen membranes on fat globules in milk that keeps it permanently emulsified, but these membranes seem to be different from caseinogen.

Measurement of surface tension is a convenient method of quantitative chemical analysis provided that only one substance greatly affecting the surface tension be variable in concentration. It is only necessary to plot the graph of surface tension and concentration in order to make a table for converting surface tension readings into concentrations. In mixtures, however, the problem may be more difficult.

G. D. Allen (1916) measured the surface tension of urine containing different concentrations of bile salts. Bile salts greatly increase the surface tension, but $\mathrm{NaCl}$, which in pure solution slightly raises the surface tension, increases the effect of bile salts in lowering the surface tension. $H$ ions increase the effect and $\mathrm{OH}$ ions decrease the effect of bile salts. 'Sodium taurocholate is slightly less effective than sodium glycocholate and, hence, a variation in the proportions of the two salts would slightly affect the result. The following table shows the limits of error when the urine is diluted to a specified specific gravity in order approximately to regulate the concentration of $\mathrm{NaCl}$ and other interfering substances.

Showing the Amounts of Sodium Glycocholate Required to Give Any Observed Surface Tension in a Sample of Urine Diluted to a Standard Sp. Gr. of I.oIO.

\begin{tabular}{|c|c|c|}
\hline $\begin{array}{c}\text { S. T. of sample diluted } \\
\text { tt } 1.010 \mathrm{sp} . \mathrm{gr} \text {. }\end{array}$ & \multirow{2}{*}{$\begin{array}{c}\text { Minimum of sodium gly- } \\
\text { cocholate which may } \\
\text { give observed S.T. } \\
\text { (Sample is acid.) } \\
\text { Diluted sample. }\end{array}$} & \multirow{2}{*}{$\begin{array}{l}\text { Maximum of sodium gly- } \\
\text { cocholate which may } \\
\text { give observed S. T. } \\
\text { (Sample is alkaline.) } \\
\text { Diluted sample. }\end{array}$} \\
\hline $\begin{array}{c}\text { Per cent iof that of dis- } \\
\text { tilled water. }\end{array}$ & & \\
\hline $\begin{array}{l}95 \\
90 \\
85 \\
80 \\
75 \\
70 \\
65 \\
60\end{array}$ & $\begin{array}{c}\text { gm. per I00 cc. } \\
0 \\
0 \\
0.001 \\
0.003 \\
0.007 \\
0.015 \\
0.033 \\
0.1+\end{array}$ & $\begin{array}{c}g m . \text { per I00 cc. } \\
0.001 \\
0.004 \\
0.008 \\
0.015 \\
0.03 \\
0.065 \\
0.1+ \\
0.1+\end{array}$ \\
\hline
\end{tabular}


Billard and Bruyant (1905) observed the use made of surface tension by the beetles, Stenus tarsalis and cicendeloides, that dart about on the surface of water. They are pulled forward by the surface tension of the water in front, while the surface tension of the water behind them is decreased by substances discharged from the anus. A model of this may be made by attaching a piece of camphor to one end of a stick floated on water. Traces of the camphor dissolve and lower the surface tension behind, and the stick moves forward. An irregular piece of camphor alone darts about owing to unequal solution on different sides.

\section{Adsorption}

As a rule, substances which lower the tension of the waterair surface also lower the surface tension between water and a solid, but the latter can be measured only in terms of adsorption. Traube gives the following data showing the effect of .25 molecular solutions on the tension of the water-air surface as compared with the amount adsorbed by a unit quantity of animal charcoal.

$\begin{array}{lcc}\text { Solute. } & \text { Surface tension. } & \text { Adsorption. } \\ \text { Acetic acid } & .84 & .244 \\ \text { Ethyl alcohol } & .85 & .136 \\ \text { Propionic acid } & .7575 & .366 \\ \text { Butyric acid } & .6 \mathrm{I} 3 & .573 \\ \text { i-Amyl alcohol } & .374 & .786\end{array}$

It may be seen that surface tension and adsorption are inversely proportional except in the case of acetic acid, which is adsorbed by charcoal more than we would expect from the surface tension of its solution against air. A similar series was obtained of the adsorption of these substances by powdered glass, cotton and silk.

The adsorption curve for different concentrations is similar to the surface tension curve, Fig. 24, a larger proportion of the solute being adsorbed from dilute solutions than from more concentrated solutions. An empirical formula to express this curve that has been suggested is $\frac{\mathrm{x}}{\mathrm{m}}=a c^{1 / n}$, where $x$ is the amount of substance adsorbed, $m$ is the amount of adsorbent powder, $c$ is the concentration of the solution after equilibrium is established and $a$ and $n$ are empirical constants (Freundlich, 1907). $\mathrm{N}$ is usually greater than I and this makes the equation characteristic 
for adsorption and surface tension. The equation is not quite correct, however, for concentrated solutions. As the concentration of the solution is increased the surface becomes saturated and will not adsorb any more.

Non-electrolytes that reduce the surface tension compete for a place at the surface. The one which is the more effective in reducing the surface tension will displace the other from the surface. The effect of two or more substances on surface tension is additive if the concentration curve is taken into consideration. The same rules hold for adsorption.

Electrolytes that reduce surface tension are adsorbed. If the surface be electrically polarized, however, it affects the adsorption of electrolytes but not of non-electrolytes. When this is the case, electrolytes and non-electrolytes that reduce the tension do not displace one another on the surface, but act independently.

The adsorbability of anions increases in the order: $\mathrm{SO}_{4}<\mathrm{Cl}$ $<\mathrm{Br}, \mathrm{NO}_{3}<\mathrm{I}$, and of cations $\mathrm{Na}<\mathrm{K}<\mathrm{Rb}<\mathrm{Cs}$ and $\mathrm{Ca}<\mathrm{Sr}$ $<\mathrm{Ba}$. The ions of heavy metals are adsorbed more than those of lighter metals; the nobler the metal the more this is true.

Neutral salts, such as $\mathrm{NaCl}$, that do not lower the tension of the water-air surface are nevertheless adsorbed by charcoal, paper, etc. The reason for this difference between the adsorption by the air and the solid surface is probably not electrical polarization, since that could not favor the adsorption of both ions of a salt, but is probably due to molecular attraction of the salt by the solid (adhesion). The adsorption of a substance by the air surface is due to the repulsion by the water molecules, but the adsorption by a solid surface might be due either to the repulsion by the water or attraction by the solid (McClendon, IgI3 b).

The electric charge of the solid, however, does influence the adsorption of ions. Clay, aluminum hydroxide and ferric hydroxide are usually electropositive, whereas kaolin, mastic, silicic acid, sulphur, charcoal, glass, silk, cotton, wool, starch grains and many other substances are negative. Acid dyes are in general adsorbed by clay but not at all by kaolin, the converse being true of basic dyes.

The electric charge of solid particles may be due to (first) electrolytic solution tension in the case of particles of metal, or 
(second) to electrolytic dissociation in the case of silicic acid which dissociates $\mathrm{H}$ ions or sulphur which partially oxidizes and dissociates $\mathrm{H}$ ions, or (third) to absorption in case the particle absorbed one ion of an electrolyte and not the other, and often (fourth) to adsorption of ions. The ions that are responsible for the electric charge of all particles are not known, but $\mathrm{H}$ ions may be displaced from positive particles by organic cations.

The electric charge of solid particles may be increased, decreased or reversed by the adsorption of ions. $\mathrm{H}$ ions and heavy metal ions make particles more positive, whereas $\mathrm{OH}$ ions make them more negative. In the neutralization of the charge of particles, bivalent ions are more than twice as effective as monovalent ions and trivalent ions are much more than three times as effective. This is what we should expect from the curve of adsorption (Fig. 24), since two molecules are not twice as effective or three molecules three times as effective as one molecule.

After reversing the charge of particles their adsorbing power is reversed. Wool will take up very little acid dye until it is made electropositive by the addition of free acid. 


\section{CHAPTER VI}

\section{ELECTROLYTES, NON-ELECTROLYTES AND COLLOIDS}

Since electrolytes in dilute aqueous solution are usually largely dissociated, it is with the ions that we have most to do. Many of the ions are elements and their properties may be predicted from their places in the periodic system. In Fig. I, curve III, the atomic weights of the elements are plotted on the abscissae and the atomic volumes on the ordinates. There are a number of U-shaped curves. At the tops of the arms are, $\mathrm{Li}, \mathrm{Na}, \mathrm{K}$, $\mathrm{Rb}$ and $\mathrm{Cs}$. These are the alkali metals and are arranged in order of their properties. The first spaces going down the left hand slopes indicate $\mathrm{F}, \mathrm{Cl}, \mathrm{Br}$ and $\mathrm{I}$. These are the halogens arranged in the order of their properties, forming the most important monatomic anions in life processes. We find $\mathrm{Be}$ (above $\mathrm{B}), \mathrm{Mg}, \mathrm{Ca}, \mathrm{Sr}$ and $\mathrm{Ba}$ occupying the first places down the right hand slopes. This is the alkaline earth series. The last three are arranged according to their physiological properties, but $\mathrm{Mg}$ sometimes behaves as an alkaline earth metal, sometimes as an alkali metal. It is peculiar also in its physical properties, $\mathrm{MgSO}_{4}$, for instance, being very poorly dissociated. The intermediate elements of the first and second curves, $\mathrm{C}, \mathrm{N}$ and $\mathrm{O}$ and $\mathrm{Si}, \mathrm{P}$ and $\mathrm{S}$, form many of the compound ions.

The most important anions may be arranged according to their effect on the thermodynamic properties of water as a solvent in the following lyotrope series: $\mathrm{CNS}, \mathrm{I},\left(\mathrm{Br}, \mathrm{NO}_{3}\right), \mathrm{Cl}, \mathrm{CH}_{3} \mathrm{COO}$, $\mathrm{SO}_{4}, \mathrm{HPO}_{4}$, Tartric, Citric. They decrease the compressibility of water from left to right. Those to the left decrease, and those to the right increase the viscosity of water. They increase the saponification of esters by bases from left to right and the reverse is true of the hydrolysis of esters by acids. They increase the surface tension of water from left to right. The electrolytic solution tension increases from left to right. The 
hydration of $\mathrm{NO}_{3}^{\prime}$ is small, of $\mathrm{Cl}^{\prime}$ is greater and of $\mathrm{SO}_{4}^{\prime \prime}$ is greatest, but the other members do not fall in order, from the available data. Their effect in salting out poorly soluble substances increases from left to right.

The ionic speed of the cations increases in the order: $\mathrm{Li}, \mathrm{Na}$, $\mathrm{NH}_{4}, \mathrm{~K}, \mathrm{Rb}, \mathrm{Cs}$ (the viscosity of their aqueous solutions decreases in same order), and the order, $\mathrm{Mg}, \mathrm{Ca}, \mathrm{Sr}, \mathrm{Ba}$. They accelerate the hydrolysis of esters by acids from left to right. The reverse is true of the hydrolysis of esters by bases. Solutions of $\mathrm{KNO}_{3}, \mathrm{KCl}, \mathrm{CsCl}$ and $\mathrm{RbCl}$ are less viscous than water. The electrolytic solution tension decreases in the order $\mathrm{Li}, \mathrm{K}$, $\mathrm{Na}$ and $\mathrm{Ba}, \mathrm{Ca}, \mathrm{Mg}$, Sr.

The heavy metals are poisonous on account of their low electrolytic solution tension, as will be discussed later.

The $\mathrm{H}$ and $\mathrm{OH}$ ions are distinguished by their very high ionic speed and relatively low electrolytic solution tensions. They are very active and distinguish the acids and bases.

\section{Non-Electrolytes}

The dissociation of the so-called non-electrolytes is so small as not to affect the osmotic pressure. The $\mathrm{H}$ ion dissociation and effect on surface tension of many of them has been given. Many of these substances may be salted out of solution by the addition of electrolytes. The order of effectiveness of ions is: $\mathrm{CNS}<\mathrm{I}, \mathrm{ClO}_{3}, \mathrm{NO}_{3}<\mathrm{Br}<\mathrm{CI}<\mathrm{OH}<\mathrm{SO}_{4}<\mathrm{CO}_{3}$, and $\mathrm{H}$ $<\mathrm{Cs}<\mathrm{Rb}<\mathrm{NH}_{4}<\mathrm{Li}$ (hydr.) $<\mathrm{K}<\mathrm{Na}$. The same holds true for salting out dissolved gases, which may be included under the non-electrolytes.

Many electrolytes and colloids may be precipitated from their water solutions by non-electrolytes. According to one view this is the substitution of one solvent for another. Salt is more soluble in water than in alcohol, and when alcohol is added to its saturated aqueous solution some salt is precipitated.

According to Traube the precipitating power of non-electrolytes is proportional to their effectiveness in lowering the surface tension of water. The following table gives their power of precipitating $\mathrm{Li}_{2} \mathrm{CO}_{3}$ as compared with the surface tension of their $.25 \mathrm{~mol}$ solutions at $15^{\circ}$ : 


$\begin{array}{lcc}\text { Non-electrolyte. } & \text { Ppt. power } & \text { Surface tension }(.25 \mathrm{~mol}) \text {. } \\ \text { Mannite } & - \text { Io.7 } & \text { I.004 } \\ \text { Dextrose } & -6.6 & \text { I.004 } \\ \text { Water } & +4.6 & \mathrm{I} \\ \text { Urea } & +9.4 & \text { I } \\ \text { Glycerol } & +998 \\ \text { Glycol } & +\mathrm{T} 4.2 & .99 \\ \text { Methyl alcohol } & +19.8 & .966 \\ \text { Ethyl alcohol } & +33.7 & .92 \\ \text { Acetonitrile } & +34.5 & .95 \\ \text { Urethane } & +4 \mathrm{I} & .87 \\ \text { Propyl alcohol } & +4 \mathrm{I} .8 & .8 \\ \text { Acetone } & +42.5 & .73 \\ \text { Pyridine } & +44.5 & .738 \\ \text { Piperidine } & +50.6 & .73 \\ \text { Ethyl ether } & +52.2 & .75 \\ \text { Diethylamine } & +56 . \mathrm{I} & .6\end{array}$

Colloids

Although Graham defined colloids as a class of non-diffusible, non-crystallizable substances, Von Viemarn claims that any substance may be made colloidal, and hence we should more correctly speak of the colloidal state. Many constituents of cells, however, are practically always in the colloidal state and are conveniently called colloids (emulsoids). Although some proteins may be crystallized, the crystals contain water and water soluble substances and are therefore not so very different from gels. The colloids are divided into suspensoids and emulsoids.

\section{Suspensoids}

The suspensoids, or suspension colloids, are ultramicroscopic suspensions. The particles are said to be liquid when first formed, but some solidify later. With the ultramicroscope the particles may be seen and their size estimated. The ultramicroscope is merely a microscope with a very powerful dark field illumination. The colloidal particles look like stars in the night except that they may appear colored, depending on their size, the smallest appearing blue. This light dispersed by colloid solutions causes them to opalesce. The light may not only be colored, but polarized as well, a phenomenon named after the discoverer, Tyndali. Even cane sugar and raffinose solutions show the Tyndall effect (De Bruyn, I900).

Whereas with the directly illuminated microscope particles .I $\mu$ in diameter may be seen, with the ultramicroscope we may 
see particles $.004 \mu$ in diameter. Since a molecule of soluble starch is calculated to be $.005 \mu$ in diameter, the limit of vision is within the size of the largest molecules. The colloidal particles visible with the ultramicroscope are called submicrons, but particles too small to see singly, amicrons, may cause a diffuse light. Since a diffuse light is caused also by submicrons which are out of focus, their presence must be excluded in order to be sure that the light comes from amicrons. Submicrons show the Brownian movement that is sometimes seen in the ordinary microscope when small suspended particles are present. The amplitude of the vibrations is inversely proportional to their size and the viscosity of the medium.

Suspensoid particles bear electric charges in the same way that the particles of a suspension do. The origin of the charges is different in different cases. It may be due to electrolytic solution tension, as in case of the negative sols (colloid solutions) of platinum, gold or iridium. It may be due to electrolytic dissociation as in case of positive sols of metallic hydroxides or basic dyes or negative sols of silicic, stannic or tannic acids, mastix or sulphonic acid dyes. Or it may be due to adsorption of ions as in case of silver iodide sol.

Colloidal particles of different sizes may sometimes be separated by means of the ultrafilter (Bechhold, I908).

Suspensoids are precipitated by ions or suspensoids of opposite charge. The isoelectric point, the point at which the particles migrate neither to the cathode nor anode, is the point of complete precipitation. Only a small amount of the precipitant is necessary and if more is added the charge on the particles may be reversed and hold them in suspension. The precipitation follows the adsorption curve (Fig. 24). Hence, bivalent ions are more than twice and trivalent much more than three times as effective as monovalent ions, but this seems not to explain entirely the difference. Among ions of the same valence the less the electrolytic solution tension and the greater the ionic speed the greater is the precipitating power. Thus negative sols are more easily precipitated by $\mathrm{H}$ and heavy metal ions. Monovalent and bivalent ions may antagonize one another in precipitation.

A precipitating electrolyte is more effective the faster it is added to the sol. The probable explanation is that when the 
electrolyte is added suddenly some colloidal particles have their charges reversed and attract others, forming larger aggregates, which are more difficult to keep in suspension. If the electrolyte be added slowly, all of the particles have the same charge, which is not reduced enough to cause precipitation. Evidently the effect of increasing the valence of the ions is the same as the effect of adding them suddenly.

Some precipitating electrolytes partially hydrolyze, forming colloidal solutions of opposite sign to the one to be precipitated. We therefore get mixed effects of electrolytic and colloidal precipitation. Thus, a negative mastix solution is precipitated by ferric chloride and also by ferric hydroxide. If a more concentrated solution of ferric chloride be added to the mastix we get precipitation by the ferric ions. If a very dilute solution of ferric chloride is added, colloidal ferric hydrate is formed by hydrolysis, but precipitation does not occur unless the sum of the charges on the hydrate exactly neutralizes the sum of the charges on the mastix. Hence the precipitating concentration is very exactly defined and varies with the concentration of the mastix sol. Since there are two concentrations of the precipitant that are effective this is called an irregular series.

Low concentrations of non-electrolytes do not precipitate.

The suspensoids are similar to suspensions in that they are precipitated by small amounts of electrolytes and the precipitate is irreversible. They do not affect the viscosity or surface tension of water, or exert osmotic pressure. This is true of dilute sols, but when very concentrated they form gels, the viscosity of which is necessarily high.

True suspensoids are probably not important in cell chemistry, but emulsoids may be changed by heat or reagents so as to assume more or less the character of suspensoids. Emulsoids so treated are said to be denatured. Thus, if a very dilute solution of egg albumin is boiled it is converted into an opalescent solution in which the particles may be seen with the ultramicroscope, and irreversibly precipitated by electrolytes. The denatured albumin particles differ from true suspensoid particles in that they contain water, but not as much as emulsoid particles. Hydroxides (such as dialyzed iron) contain some water.

Living cells may be so changed as to resemble the particles 
of a suspension or suspensoid sol, although they are probably always killed in the process. The process of agglutination of bacteria consists of two stages: first, the transformation of the bacteria into suspensoid particles by treatment with specific agglutinins or by boiling; second, the precipitation of these particles by electrolytes. The precipitation of a suspensoid sol is always inatgurated by clumping of the particles.

Cholesterin and uric acid may form suspensoid sols and gels. Cholesterin appears to form a true solution in the bile, however, and it cannot be detected within the red blood corpuscles by means of the ultramicroscope.

Lecithin when rubbed up with water forms a sol which is intermediate between a suspensoid and an emulsoid. It resembles an emulsoid in that the particles contain water. It resembles a suspensoid in that the particles may be easily seen with the ultramicroscope. Within the red blood corpuscles, however, the lecithin cannot be seen with the ultramicroscope and evidently forms part of the emulsoid gel, either due to the difference in the medium or to the fact that it is chemically combined with one or more of the other substances present.

\section{Emulsoids}

The emulsoids are called hydrophile colloids by Perrin because of their great affinity for water. They are the true colloids, or glue-like substances, according to Graham's definition, except for the fact that some of them may be crystallized with more or less difficulty. When a dry mass of colloid substance is placed in water it absorbs water and swells, with the evolution of heat, forming a gelatinous mass. It usually requires mechanical or other assistance, however, to get it into solution. The colloid particles in sols contain water and the water between them contains some dissolved colloid. It is probably for this reason that the particles do not appear distinct with the ultramicroscope. An absolutely pure solution of gelatine appears clear with the ultramicroscope except at certain concentrations and temperatures, and if some particles are seen in it they probably are impurities. Under certain conditions, however, the particles lose some of their water and approach in character the suspensoids, becoming optically more distinct. Similarly, in an absolutely 
pure gelatine jelly or gel, no structure can be seen with the ultramicroscope except at certain concentrations and temperatures, whereas in a suspensoid gel the arrangement of particles into chains and the chains into a network may be distinguished. According to Zsigmondy (I9I2), I/2 per cent gelatine shows submicrons on cooling, first mobile but becoming motionless during gelation. The evidence for a structure in a gel is the sudden increase in elasticity during gelation. A gel is not merely a very viscid sol, because agents that increase viscosity induce solation. Fibrin gel seems to be a mass of crystals (W. H. Howell, I9I4).

The emulsoids are probably to be regarded as true solutions, in which the molecules are very large and sometimes aggregated into masses that include more or less water. Some colloids probably have a stronger tendency to aggregate than others. Hemoglobin seems to have little of this tendency when in not too concentrated a solution. Hüfner and Gansser (I907) conclude that it is in true solution. If it be assumed that one molecule of hemoglobin contains one atom of $\mathrm{Fe}$ and binds one molecule of $\mathrm{CO}$, its molecular weight is about 16700 . Calculated from the osmotic pressure of pure solutions, the molecular weight of horse hemoglobin is I5II5, and of ox hemoglobin I632I. Since one molecule of hemoglobin cannot contain less than one atom of iron, the molecules must be separated in solution in order to exert so high an osmotic pressure.

Bechhold (I908) has attempted to estimate the size of the particles in emulsoid sols by forcing them through ultrafilters of different grades. These filters are made by impregnating filter paper with an emulsoid sol and hardening it. The size of the pores is determined by the concentration of the sol before being hardened. There are two difficulties with the method: first, the filter may adsorb the colloid, second, some water may be pressed out of the sol in the filter and increase its concentration with enormous increase in viscosity until the flow is stopped. Notwithstanding these difficulties Bechhold has arranged emulsoids in the following series beginning with those with the largest particles: 
Caseinogen (in milk)

Gelatine (I per cent)

Hemoglobin ( 1 per cent)

Serum albumin

Protalbumoses

Duteroalbumoses A

Deuteroalbumoses B

Dextrin

Emulsoids enormously increase the viscosity of water. One per cent gelatine increases the viscosity of water 29 per cent.

Emulsoids reduce the surface tension and are therefore adsorbed. They become denatured in the surface film to the extent that they no longer go into solution in some cases (haptogen membranes). In this way they may be precipitated by shaking the solutions. The surface films are submerged and distorted, forming stringy masses, while new surface films are formed. Emulsoids adsorbed by solids cannot be washed out with water because they have become insoluble. Emulsions of oil and water are permanent if colloids are present as the haptogen membranes formed about the oil drops prevent them from fusing. Soap acts in this way. Some colloid from milk glands forms haptogen membranes around the fat droplets, but it is said not to be caseinogen. The plasma membrane on the surface of protoplasm is supposed to be of this nature although the colloids in its composition have not been ascertained. Presumably, the colloids of the cell: proteids, lecithins, and cholesterin, enter into its composition.

Emulsoids are not precipitated by small amounts of electrolytes unless they have been denatured. They may be salted out by large amounts of neutral salts in the same way that truly dissolved substances, such as gases or alcohol, may be salted out of their aqueous solutions. The electric charge of the colloid is not entirely without significance in the salting out process, however, as will be seen in the anionic series. The order of effectiveness of anions in salting out soluble non-electrolytes is (beginning with the least effective): $\mathrm{CNS}, \mathrm{I}<\mathrm{NO}_{3}<\mathrm{Cl}$ $<\mathrm{CH}_{3} \mathrm{COO}<\mathrm{SO}_{4}<$ Tartrate $<$ Citrate (Hofmeister, I89I). The order of effectiveness with negative emulsoids is the same, 
but the order is reversed in case of positive emulsoids. The order of effectiveness of cations in precipitating negative emulsoids is $\mathrm{Li}<\mathrm{Na}<\mathrm{K}<\mathrm{Rb}<\mathrm{Cs}$, and with positive emulsoids the reverse.

There is not much difference in the precipitating power of the ions of alkali metals, but the bivalent cations (except $\mathrm{Mg}$ ) precipitate emulsoids much more effectively than monovalent cations, and trivalent cations more effectively than bivalent. The effectiveness of the heavy metals and sometimes $\mathrm{Ca}, \mathrm{Sr}, \mathrm{Ba}$ and $\mathrm{H}^{*}$ in precipitating emulsoids lies partly in the fact that they denature the emulsoids, making them easier to precipitate. Such precipitates are irreversible, whereas the precipitations with neutral salts of the alkali series are reversible.

Neutral salts reduce the osmotic pressure of emulsoid solutions, presumably by causing the particles to aggregate and form larger ones, thus reducing the number. The order of effectiveness of the anions is the same as given above.

Emulsoids are precipitated by some non-electrolytes, such as alcohol (see non-electrolytes).

Many of the emulsoids are proteids and are amphoteric. These are made electropositive by acid and negative by alkali. There is probably a chemical reaction of the nature: $\mathrm{Alb} \cdot \mathrm{OH}+\mathrm{HCl}=$ $\mathrm{Alb} \cdot \mathrm{Cl}+\mathrm{HOH}$, after which the acid albumin dissociates into $\mathrm{Alb}^{\bullet}$ and $\mathrm{Cl}^{\prime}$; and $\mathrm{Alb} \cdot \mathrm{H}+\mathrm{KOH}=\mathrm{Alb} \cdot \mathrm{K}+\mathrm{HOH}$, after which the alkali albuminate dissociates into $\mathrm{Alb}^{\prime}$ and $\mathrm{K}^{\circ}$.

Small amounts of acid or alkali increase the osmotic pressure and viscosity, decreasing the surface tension of albumin solutions. This is probably due to the fact that the acid and alkali albuminates are more soluble or highly hydrated in the sense that the molecules are not aggregated and hence the "molecular concentration" is increased. The addition of larger amounts of acid increases the effect up to a certain limit, above which the albumin is denatured.

Since the proteins are amphoteric they dissociate both $\mathrm{H}$ and $\mathrm{OH}$ ions, and are either positive or negative according to whether they dissociate more $\mathrm{OH}$ or more $\mathrm{H}$ ions. According to the law of mass action, the greater the concentration of the $\mathrm{H}$ ions in the solvent the less $\mathrm{H}$ ions will be dissociated, and the greater the concentration of $\mathrm{OH}$ ions (or the less the concentration of 
$\mathrm{H}$ ions) in the solution the less will be the $\mathrm{OH}$ ion dissociation by the protein. Therefore, at a certain $\mathrm{H}$ ion concentration of the solvent the $\mathrm{H}^{*}$ and $\mathrm{OH}^{\prime}$ dissociation of the protein will be equal, and the protein will migrate neither to the cathode nor to the anode in an electric field. This is called the isoelectric point of the protein, at which the protein can be most easily precipitated (by alcohol, for instance). At this point the viscosity of the sol is at its minimum.

The albumins, globulins and some other proteins are more acid than basic. The isoelectric points of some of the proteins as determined by Michaelis (I9I2) are as follows:

Protein $\quad \mathrm{PH}$

Serum albumin .................... 4.7

Serum globulin $\ldots \ldots \ldots \ldots \ldots \ldots \ldots \ldots, 5.4$

Casein $\ldots \ldots \ldots \ldots \ldots \ldots \ldots \ldots \ldots \ldots, 4.7$

Gliadin $\ldots \ldots \ldots \ldots \ldots \ldots \ldots \ldots \ldots, 9.3$

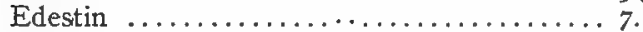

Oxyhemoglobin $\ldots \ldots \ldots \ldots \ldots \ldots \ldots \ldots .6 .74$

Pancreasnucleoprotein (trypsin) ......... 3.52

From typhus bacilli .................. 4.4

From paratyphus bacilli ............... 4 .

The isoelectric point of sols is also the point of least hydration, viscosity and stability, and of greatest surface tension. The size of the colloid particles is probably largest at this point.

In the precipitation of emulsoids as well as suspensoids, monovalent and bivalent cations are antagonistic to one another.

Emulsoids are precipitated by suspensoids, but if too much of the emulsoid is present for precipitation it forms coatings on the suspensoid particles and prevents their being precipitated by small amounts of electrolytes. In order to obtain complete precipitation, the suspensoid must be of opposite electric charge to the emulsoid.

Emulsoids may precipitate one another even when of the same sign electrically. Thus nucleic acid precipitates other proteins and specific precipitins precipitate the precipitable substances, both being of the same sign (cf. Teague and Buxton, I907). According to Michaelis, the ratio between precipitin and precipitable substance in the precipitate is not constant, and is not therefore a chemical reaction, but most probably an adsorption phenomenon.

The time element in colloid phenomena is little understood. A 
less concentration of the precipitant is necessary if it be added suddenly than if added gradually. A less amount of antitoxin is necessary to render a toxin solution inert when added at once, than when added gradually. On the other hand, time is favorable to the increase of viscosity. Gels become firmer with age and haptogen membranes likewise, even though perfectly protected from evaporation. This phenomenon is known as hysteresis or secondary change.

The gels may be considered very viscous sols but the cause of the great viscosity probably lies in the supposed structure. The swelling of gels or gelatine plates may be made to produce a pressure which is thought, by some authors, to be analogous to the osmotic pressure of sols. The pressure is increased by acids and alkalis. The emulsoid gel may be converted into a suspensoid gel in the same way that an emulsoid sol is converted into a suspensoid sol by coagulative agents. Some sulphates and tartrates cause gelatine gels to shrink, bromides and nitrates causing them to swell (Hofmeister, I888). According to Lenke (I9I6) these two groups differ in that with the first there is an optimum concentration for swelling and with the second the greater the concentration the greater the swelling. Their effect on gelation is the inverse of their effect on swelling, the series being: $\mathrm{SCN}<\mathrm{Br}, \mathrm{NO}_{3}<\mathrm{Cl}<\mathrm{CH}_{3} \mathrm{COO}<\mathrm{Cit}$. $<$ Tart. $<\mathrm{SO}_{4}$. Glycerine, dextrose and saccharose increase the viscosity of gelatine and starch gels, and urea decreases it. The reverse is true of their effect on swelling.

The ionic series which holds for negative emulsoids and is reversed for positive, becomes irregular as we near the isoelectric point. This explains the variations in the ionic series found in various physiological experiments since the emulsoids in living cells are near the isoelectric point.

According to Pauli, anions increase swelling of gelatine gels and cations decrease it, the greater the atomic weight of the cation, the greater its effect.

Traube (I9I5) claims that the more a substance hastens solation (solution of a gel), the more it favors the rate and amount of swelling, the more it hastens gelation, the more it disfavors the rate and amount of swelling. He finds that the stronger acids hasten gelation when very dilute $(\mathrm{PH}=$ isoelectric point $)$ 
and that there is a maximum retardation at about $.02 \mathrm{n}$ at which Fischer observed the maximum swelling, and Pauli and Wagner (I9IO) observed the maximum viscosity (of serum albumin in the last case). Traube claims that $\mathrm{Ca}^{\circ}$ and $\mathrm{K}^{*}$ hasten gelation and $\mathrm{Na}^{\circ}$ retards it and therefore $\mathrm{Ca}^{\circ}$ antagonizes $\mathrm{Na}{ }^{\circ} . \mathrm{He}$ claims that anesthetics retard gelation (see Chapter XI).

Chiari (I9II) found the least swelling of gelatine to occur when the $\mathrm{PH}=4.7$, which corresponds closely to the isoelectric point, $\mathrm{PH}=4.6$ as determined by Michaelis. No other investigator of the effect of acids and alkalis on emulsoids has determined the $\mathrm{PH}$. 


\section{CHAPTER VII}

\section{ENZYME ACTION}

Enzymes are classified according to their action, and not according to their constitution. Although Berzelius defined catalyzers as those substances which by their mere presence cause reactions to take place between other substances, many catalyzers are now known to take part in the reactions. Ostwald supposed that catalyzers accelerate the rate of only those reactions which go on at a slower rate in the absence of the catalyzer. It is true in certain cases, however, that the catalyzer may change the point of equilibrium of a reversible reaction. An example of this was observed by Bodenstein and Dietz (1906) in the reversible decomposition and formation of amyl butyrate. In the presence of an excess of water and amyl alcohol, the same equilibrium point was reached whether the amyl butyrate or the amyl alcohol and butyric acid were present in the beginning. But if pancreas lipase were used as a catalyzer, 75 per cent of the ester was formed or left against 85 per cent of the ester when $\mathrm{HCl}$ was used as a catalyzer.

The chief distinction that is supposed to separate enzymes from other catalyzers is their specificity. This is perhaps overrated, but is fairly well established in some cases. Amygdalin may be decomposed by means of emulsin into $\mathrm{HCN}$, benzaldehyde and dextrose. Emmerling (I90I) showed that this took place in three stages, each of which was accelerated by a different enzyme, and that all three enzymes were present in emulsin. The reversal of the process could be brought about by applying the enzymes in a certain order to the separate stages.

In case of stereoisomeres or optically active isomeres, an enzyme may accelerate a reaction involving one isomere more than the other. If $\mathrm{HCN}$ and benzaldehyde are mixed in solution optically inactive benzaldehydecyanhydrin is formed, but if the reaction is accelerated by means of emulsin, d-benzaldehyde- 
cyanhydrin is formed. E. Fischer (I898) observed that betamethyl-d-glucoside is split by emulsin whereas alphamethyl-dglucoside is not.

This form of specificity is not peculiar to enzymes. According to Bredig and Fajans (1908) the splitting of camphocarbonic acid into camphor and $\mathrm{CO}_{2}$ is accelerated by certain organic compounds. Natural nicotine decomposes d-camphocarbonic acid I2 per cent faster than 1-isomere. Chinin decomposed the 1-isomere 46 per cent faster than the $\mathrm{d}$-isomere.

The specificity just mentioned is quantitative and not qualitative, whereas the specificity of enzymes is supposed to be qualitative. It was shown by H. D. Dakin (1905), however, that the specificity of lipase for certain d-esters in preference to the isomeric l-esters, is quantitative.

More unexpected than the specificity of enzymes, is the change in specificity on reversing the reaction. A. Croft Hill (1898) succeeded in synthesizing a disaccharide from dextrose by means of maltase from yeast. Emmerling (I9OI) showed that this disaccharide is not maltose, as was to be expected, but isomaltose. E. F. Armstrong (I903) continued this investigation and found that:

whereas maltose is split into glucose by maltase glucose is synthesized to maltose by emulsin, and whereas isomaltose is split into glucose by emulsin glucose is synthesized to isomaltose by maltase.

The explanation of this anomaly is that the enzymes change the point of equilibrium of these reversible reactions. In a mixture of maltose and glucose, maltase displaces the equilibrium toward the side of the glucose, whereas emulsin displaces it toward the side of the maltose. If $\mathrm{HCl}$ is used as a catalyzer, when equilibrium is established glucose, maltose and isomaltose are present in the same proportions, irrespective of the first used. If glucose only is present at the start, and maltase is used, very much more isomaltose than maltose is formed, but if emulsin is used, much more maltose than isomaltose.

Since enzymes merely accelerate reactions that would take place without them and all reactions are theoretically reversible, the synthesis of complex bodies from their decomposition pro- 
ducts might be expected with the aid of the same enzymes that affected the decomposition. Hill was the first to accomplish synthesis with enzymes, and obtained a different compound from the original. Kastle and Loevenhart (I900) succeeded in synthesizing a fat, ethyl-butyrate, from butyric acid and alcohol, by means of lipase of the pancreas. The attempts to synthesize proteins have not given such unequivocal results. Since the chemical constitution of proteins is unknown, it is not surprising that some difference of opinion should arise in the interpretation of changes occurring in mixtures of protein decomposition products and protease. Some interpret the appearance of a precipitate as synthesized protein, others as coagulation of albumoses already present. Perhaps one difficulty in producing syntheses is that they are likely to be endothermic reactions, which absorb energy from without, as in case of the synthesis of carbohydrates in the chloroplast of the green plant under the influence of sunlight. The energy of the light is absorbed during the synthesis. In this process the $\mathrm{CO}_{2}$ is reduced and hydrated with the formation apparently of formaldehyde, which is synthesized into sugar and starch.

As was previously stated, the important effect of a catalyzer is on the rate of a reaction. In case the concentration of the catalyzer remains constant, implying that if it enters into the reaction, a vanishing quantity of it is bound at any one time, the rate of the reaction may follow the law of mass action for a monomolecular reaction.

$$
-\frac{d x}{d t}=c(a-x)
$$

In which $a-x$ is the concentration of the substance at any particular moment, and $c$ is the constant denoting the speed of reaction. This law holds true, for instance, for the hydrolysis of cane sugar by means of a fixed $\mathrm{H}$ ion concentration, since the concentration of the $\mathrm{H}_{2} \mathrm{O}$ that enters into the reaction remains the same, and the only variable is the concentration of the cane sugar. If the concentration of the $\mathrm{H}$ ions is varied, the reaction constant, $c$, varies in the same ratio.

One of the oldest theories of the mechanism of catalytic action is that the catalyzer enters into the reaction transforming it into 
two stages, the sum of which takes less time than the uncatalyzed reaction. In this way is explained the action of nitrous oxide in the oxidation of sulphurous acid, and the action of sulphuric acid in the conversion of alcohol into ether.

The catalyzer may change the course of the reaction, as in the observation of Slator (1903) on the chlorination of benzol, in which the $\mathrm{Cl}$ may be substituted or simply added. Different catalyzers favor one or the other form of combination. This is illustrated in enzymes by the difference in the form of proteolysis produced by pepsin and by trypsin.

In case of reactions in a heterogeneous system, of which a diphasic system is the simplest case, not only the rate of the chemical reaction but also the rate of diffusion may enter into the rate of transformation of the substance.

In the first case, the second phase may be a better medium for the reaction than the first phase, and so act as a catalyzer. Bredig (I9OI) showed that methyl acetate dissolved in benzol is not saponified, even with the addition of triethylamine. If the mixture is shaken with 2.5 per cent water to form an emulsion, saponification takes place with great rapidity, because the water dissociates the triethylamine, and the resulting $\mathrm{OH}$ ions catalyze the saponification of the methyl acetate. The same principle seems to be involved in the catalysis of the union of hydrogen and oxygen by means of palladium or other noble metals. The palladium dissolves these gases, forming a so-called solid solution. Hoitsema (1895) has shown that the amount of hydrogen dissolved by the palladium is proportional to the square root of the hydrogen pressure, and the hydrogen, therefore, does not exist in the metal as $\mathrm{H}_{2}$ but as $\mathrm{H}$. If this atomic hydrogen is more active than molecular hydrogen, the catalytic action of the palladium is explained.

The second phase of a diphasic system may increase the rate of reaction by adsorption. Bayliss (I9I5 b) has shown that enzymes may act in a medium in which they are insoluble. We shall consider this aspect of the subject under the head of oxidation (Chapter XV).

In case the rapidity of the chemical reaction in or on the second phase is very great, perhaps too great to measure, the rate of transformation is determined by the rate of diffusion 
in the first phase toward the second. This seems to be the case with many reactions catalyzed by finely divided platinum and is supposed to be a factor in enzyme reactions, since enzymes seem to be of colloidal nature.

Catalysis with colloidal solutions of precious metals resembles the action of enzymes in many ways. Both have a temperature optimum, that is, both are rendered inactive by boiling and even lower temperatures. According to Ernst (I9OI) the temperature optimum for the oxidation of hydrogen under the influence of platinum sol lies between $65^{\circ}$ and $85^{\circ}$. Both are affected by the reaction of the medium. The decomposition of $\mathrm{H}_{2} \mathrm{O}_{2}$, either by platinum sol or by the enzyme, catalase, is favored by a slightly alkaline reaction. Both the catalase and oxidase action of enzymes or colloidal precious metals is suspended by $\mathrm{HCN}$ and returns when the $\mathrm{HCN}$ is removed, if just enough were used to suspend action. The same is true of the action of $\mathrm{H}_{2} \mathrm{~S}$. On the sols of $\mathrm{Cu}$ and $\mathrm{Fe}$, however, $\mathrm{HCN}$ increases catalytic activity, according to Kastle and Loevenhart (I903). Finally, Meyerhof ( I9I4 b) has shown that the action of platinum sol on $\mathrm{H}_{2} \mathrm{O}_{2}$ is reduced by the presence of anesthetics, in the same way that enzyme actions are reduced by anesthetics. The anesthetic forms a protective film on the platinum particles, due to adsorption.

The importance of the reaction of the medium, that is, the $\mathrm{H}$ ion concentration, on the activity of enzymes has long been known. Michaelis (I9I4) attempts to explain this action by assuming the enzymes to be weak electrolytes, i. e., acids, bases or ampholytes, whose dissociation is affected by the $\mathrm{H}$ ions. At the optimal $\mathrm{H}$ ion concentration the activity of the enzyme solution is proportional to the concentration of the enzyme and all of the enzyme is in the active form. At any other $\mathrm{H}$ ion concentration some fraction of the enzyme is in an inactive form, and therefore the total activity is reduced.

According to Sörensen (I909) and Michaelis and Davidsohn (IgII) the optimal PH for the invertase of yeast is about 4.3. Above the optimum the activity of the enzyme decreases to zero at about 9, whereas below it the activity decreases (because the enzyme is destroyed?) so that little action is obtained below 3 or 2 , depending on the duration of the experiment. Michaelis interprets this as follows: The enzyme is an ampholyte with 
the isoelectric point at the optimal $\mathrm{H}$ ion concentration. Hence, it is the undissociated molecules of the invertase that are active. Above the isoelectric point (alkaline side) the invertase dissociates as an acid, and the activity curve corresponds to the curve for the undissociated part of an acid. Below the isoelectric point (acid side) the invertase dissociates as a base, but the invertase cations are very unstable and soon become denatured, so that the activity of the enzyme is not restored by return to the isoelectric point.

In the same way Michaelis concludes that trypsin and erepsin are acids whose anions are proteolytic. Whether the theory of Michaelis is right or wrong, the relation of the $\mathrm{H}$ ion concentration to the activity of enzymes is no less important. The following table gives the optimal $\mathrm{H}$ ion concentration for the action of several enzymes ( $\mathrm{McClendon,} \mathrm{I9i6} \mathrm{b).}$

\begin{tabular}{|c|c|}
\hline Enzyme & Optimum PH. \\
\hline Amylase, pityalin & 6.7 \\
\hline $\begin{array}{l}\text { ataliase of blood and liver } \\
\text { Cellase }\end{array}$ & $\begin{array}{l}7 \\
6 .\end{array}$ \\
\hline $\begin{array}{l}\text { Glycolase of blood } \\
\text { "Invertase) }\end{array}$ & $\begin{array}{l}7.52 \\
4.5\end{array}$ \\
\hline $\begin{array}{l}\text { Maltase (maltose) } \\
\text { (alphamethylglucoside }\end{array}$ & $6.6\left(3\right.$ at $35^{\circ}, 7.2$ at $\left.47^{\circ}\right)$ \\
\hline $\begin{array}{l}\text { nulase } \\
\text { ippe of stomach }\end{array}$ & $\begin{array}{l}3 . \\
6.7\end{array}$ \\
\hline “" " Ricinus & 3. \\
\hline $\begin{array}{l}\text { Xidase, laccase } \\
\text { rotelase, erepsin }\end{array}$ & 7.8 \\
\hline " (plastein or rennin & \\
\hline $\begin{array}{l}\text { (plastein iormation) } \\
\text { trypsin (peptone) }\end{array}$ & 7.7 \\
\hline "s (gelatinle) & 9.7 \\
\hline bacterial & 7.2 \\
\hline of malt & 4.5 \\
\hline Jrease (comb. with urea). & $\begin{array}{l}5.7 \\
8.7\end{array}$ \\
\hline derommosition & 7. \\
\hline
\end{tabular}

The optimum PH for enzyme action is of interest in connection with the reaction of the parts of the alimentary tract, where the digestive enzymes act.

The saliva is generally supposed to be alkaline, but according to Michaelis (I9I4) it has a $\mathrm{PH}$ of about 6.87 , which is near the optimum for the action of ptyalin (6.7). 
The optimum PH for pepsin is, according to Sörensen (I909), I.5-2, and the results of Michaelis and Davidsohn fall between these limits. Sörensen observed considerable digestion at $\mathrm{PH}=0.7$ near the maximum acidity and $\mathrm{PH}=5$ near the minimum acidity. From curves showing the rise in acidity of the stomach measured with the hydrogen electrode (McClendon, I9I5 e) it may be observed that the acidity becomes high enough for digestion to commence during the first hour and the optimum is reached by about the end of the second hour if at all. Menten (I9I5) found the PH of pure human gastric juice to vary from 0.92 to 1.58 , corresponding to an average of .36 per cent HCl. Apparently the reaction of the stomach is determined by the amount of food in it and the rate of flow of the juice. The juice secreted into an empty stomach would have a very high acidity, but Boldyreff (I9I5) has shown that it is lowered by regurgitation of pancreatic juice. The $\mathrm{PH}$ in hyperacidity, from the figures given by Michaelis (I9I4), indicates not nearly so high an acidity as the pure gastric juice. It seems probable that hyperacidity is brought about by closure (spasm) of the pylorus, preventing removal or neutralization of the acid.

The PH of the pure gastric juice of the infant is about 2.3 (McClendon, 19I5 e), being nearly the optimum for the action of pepsin, which it contains, but the juice is apparently secreted so slowly that the $\mathrm{PH}$ is $5 \cdot 3$, while the stomach is full of milk, which is about the optimum for the action of rennin (pepsin?) in coagulating milk.

The $\mathrm{PH}$ of the adult duodenum is about 7.7 or the optimum for the action of trypsin and erepsin on peptones.

In the infant a very peculiar state of affairs exists. As the milk leaves the stomach the acidity rises until it equals that of the pure gastric juice about four hours after nursing. The neutralizing power of the pancreatic juice is very low, so that the duodenum has about the same reaction as the pylorus. The $\mathrm{PH}$ of the duodenal content is usually about 3.I and pepsin is present, so that peptic digestion must take place in it.

It has been shown by a number of observers that the rate of enzyme action does not always vary in direct ratio with the concentration of the substance to be transformed, substrate, but that high concentrations of the substrate are relatively detrimental. 
Many hypotheses have been made to account for this anomaly. An increase in the substrate concentration may increase the viscosity and therefore decrease the speed of diffusion. An increase in the beginning concentration of the substrate means an early accumulation of reaction end products which may be detrimental to the reaction, either by starting a reaction in the reverse direction or by combination with the enzyme. This hypothesis is supported by the fact that the spliting of cane sugar by invertase is retarded more by fructose than by glucose, and mannose or glycerine have about the same inhibiting activity as glucose. It has been supposed that the enzyme may become saturated with the substrate, based on the fact that in some cases the rate of reaction depends on the concentration of the enzyme more than on the concentration of the substrate.

In some cases the products of the reaction activate the enzyme and hence the rate of reaction constantly increases. This type of reaction is called autocatalytic, but it is to be remembered that it does not catalyze itself in the sense of furnishing an enzyme, but increases the rate by activating an enzyme already present. Thus, alcoholic fermentation either with living or dead yeast increases with the accumulation of the end products up to a certain point. Hoyer (1907), in studying the saponification of castor oil during the autolysis of ground castor beans, observed that the reaction proceeded slowly until the $\mathrm{H}$ ion concentration reached a certain point, when it suddenly increased. If the $\mathrm{H}$ ion concentration were increased at the start by the addition of acids, the splitting of the oil was rapid from the first. The higher fatty acids produced by the splitting of castor oil were probably not sufficiently soluble to produce the required $\mathrm{PH}$ alone, and were aided by $\mathrm{CO}_{2}$ and lactic acid appearing in the mixture. But we may imagine the splitting of the ester of a lower fatty acid by the same enzyme, in which the fatty acid produced would cause the rate of reaction to increase. This might be called autocatalysis. According to M. Morse (1916) the autolysis of ground animal tissues is autocatalytic, since the rate of proteolysis is proportional to the $\mathrm{PH}$ which constantly increases during the reaction in a characteristic curve. Bradley and Taylor (1916) conclude that $\mathrm{H}$ ions act not on the enzyme but on the substrate, increasing the number (and consequently the total surface area) of the colloid particles. 


\section{CHAPTER VIII}

\section{PERMEABILITY OF CELLS}

It was shown by Nägeli, Pfeffer and de Vries that concentrated solutions of neutral salts cause plant cells to shrink by extracting the water from them. The shrinkage, plasmolysis, of plant cells may be easily detected because, though the cell wall does not shrink, the shrinkage of the protoplasm separates it from the cell wall, leaving a space between the two. Solutions with equal plasmolytic power de Vries (I884) called isotonic solutions, and showed a relation between their percentage concentrations and the molecular weights of the solutes. This was the foundation of van't Hoff's theory of osmotic pressure. The discrepancies in the relation between the concentration of isotonic solutions and the molecular weight of the solutes was explained by Arrhenius on the assumption that electrolytic dissociation increases the number of particles in solution. In this way a method was developed of measuring the osmotic pressure of plant cells. The osmotic pressure of solutions that just fail to plasmolyze the cells is equal to the osmotic pressure of the cells. If animal cells are used, some method of determining the first decrease in volume must be used. When the cells are in large masses, changes in volume may be determined by weighing the masses. If the cells are free, as with blood corpuscles, their volume may be determined by centrifuging them in graduated tubes (Hamburger, I893).

Some substances do not plasmolyze cells no matter how great the concentration, due to the fact that the cells are permeable to the substances which consequently cannot exert any osmotic pressure on the cells. Others cause only a temporary plasmolysis because they slowly penetrate the cells. When the concentration becomes the same outside and inside they cease exerting osmotic pressure.

The question arises as to whether the whole cell substance, 
or only the surface, is impermeable to salts. The protoplasm of most plant cells forms only a thin surface layer, the primoidal utricle, the interior being filled with cell sap which is an aqueous solution of many substances. Young plant cells, and all animal cells, have only small or no vacuoles filled with cell sap, and little or no nuclear sap, whereas mammalian erythrocytes are entirely composed of a homogeneous gel. In the plant cell, at least, only the surface layer can be the impermeable portion, and Pfeffer found evidence that not all of the primoidal utricle, but only the surface film, is impermeable. Höber showed that only the surface of erythrocytes is impermeable to ions. His methods are complicated, as will be seen from the following paragraphs.

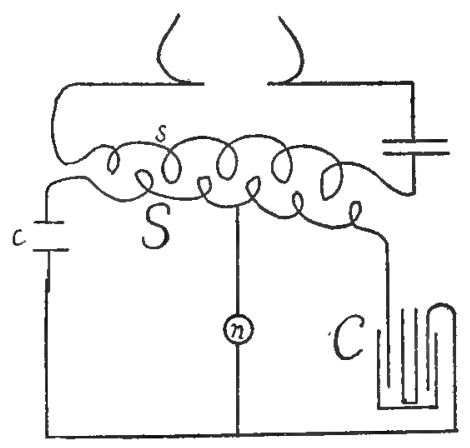

FIG. 25. Scheme for estimating the electric conductivity of cell interiors by the increase in capacity of a condenser into which the cells are introduced (from Höber, 1914).

It was shown by G. N. Stewart that erythrocytes do not conduct the electric current, and are therefore impermeable to ions, unless they are laked. Höber showed that ions move freely in the interior of the erythrocytes notwithstanding the fact that they cannot get in or out. This was first done by the "capacity method" (IgIO a). The capacity of an electric condenser is increased if a conducting body is introduced into the dielectric between the two plates of the condenser, the increase being greater the greater the electric conductivity of the body. The apparatus consists of a primary coil ( $s$, Fig. 25), through which high frequency electric oscillations are passed, such as are used 
in wireless telegraphy. These oscillations induce similar ones in the secondary coil, $S$, which is connected at one end with the adjustable condenser, $c$, and at the other with the condenser, $C$, between the plates of which the material to be investigated is placed. The other plates of these condensers are connected, and the circuit bridged by an instrument, $n$, for detecting a current, according to the principle of the Wheatstone bridge. If the capacity of $c$ does not equal that of $C$ some current will flow through $n$. The plates of $c$ are separated by such a distance that no current flows through $n$, which means that the capacities of $c$ and $C$ are equal. A test tube filled with the erythrocytes is placed between the plates of $C$. If the capacity of $C$ is thereby increased, the plates of $c$ would have to be approximated in order that no current flow through $n$. In order to prevent error due to the conductivity of the fluid wetting the outside of the corpuscles, they are washed repeatedly with isotonic sugar solution. By filling capsules corresponding in size to the corpuscles with solutions of different conductivity, and finding the conductivity of the solution which causes the same increase in the capacity of $c$ as was caused by the blood corpuscles, the conductivity of the interior of the corpuscles may be determined. Höber did not make his capsules of conducting solutions as small as the blood corpuscles nor consider the question of size at all. $\mathrm{He}$ calculated that the conductivity of the interior of the corpuscles is between that of a .I and a .OI normal $\mathrm{KCl}$ solution.

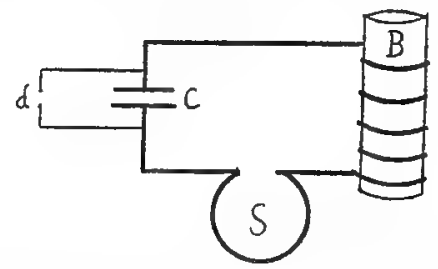

FIG. 26. Scheme for estimating the electric conductivity of cell interiors by the damping of the inductance of a coil in the core of which the cells are placed (from Höber, I9I4).

Another method for measuring the internal conductivity is the damping method (Höber, I9 5 b). In Fig. 26, $S$ is the secondary coil in which high frequency electric oscillations ( IO $^{7}$ a second) 
are induced, $B$ a coil around a beaker, $c$ a condenser, and $d$ a detector with which to observe any change in the oscillating current. If the beaker is filled with a conducting solution the oscillations are damped, as shown by the detector. If erythrocytes are placed in the beaker the oscillations are damped as much as by 0.4 per cent $\mathrm{NaCl}$ solution. This remains the same if the plasma membranes are destroyed by saponin. Since the conductivity, as measured by the Kohlrausch method, is increased ten times by the destruction of the plasma membranes, they seem to be the chief cause of the low conductivity of the normal erythrocytes.

A third method for measuring the internal conductivity of cells is the use of high frequency electric oscillations instead of induction coil currents, in the Kohlrausch method (Höber, $1913 \mathrm{~b})$. When an alternating current is passed through a solution, the cations move in one direction until the current changes, then move in the opposite direction, arriving at their original positions. The anions behave similarly. The distance traveled by an ion is proportional to the duration of one phase of the current, or inversely proportional to the frequency of the alternations. The higher the frequency the less the distance traveled by an ion. When the frequency is ten million per second the distance traveled by an ion is insignificant. The plasma membranes stop very few ions directly, but stop or retard all of them by the electric polarization produced by the stoppage of a few ions. The less the distance that would be traveled by an ion, the less the polarization, hence the very slight polarization when high frequency oscillations are used. The conductivity measured by means of high frequency oscillations is therefore practically the same, whether the plasma membranes are present or absent. It should be noted, while this line of reasoning is in mind, that the conductivity of erythrocytes, measured by the Kohlrausch method, using an induction coil with rapid interrupter, is too high, and it is theoretically only with a direct current and nonpolarizable electrodes that the true conductivity may be measured. In actual practice the errors with the direct current are usually so great that the Kohlrausch method is to be preferred.

By means of the damping method, Höber found the internal conductivity of frog's muscle that had been washed six hours in 
isotonic sugar solution, to be equal to that of a 0.15 per cent $\mathrm{NaCl}$ solution, whereas the Kohlrausch conductivity $=0.03$ per cent $\mathrm{NaCl}$. It seems probable, therefore, that the chief impermeable layer of all cells is the plasma membrane, although other membranes, nuclear membranes and vacuole membranes, may also be impermeable to many substances.

These experiments of Höber are selected because the cells are not injured in the least. If space permitted, a large number of experiments which go to show that the plasma membrane is the semipermeable part of the cell could be described. The word semipermeable was first used to describe permeability to water and to nothing else. This is never true of the plasma membrane, since it is always permeable to oxygen, for instance. The word is now used to denote limited permeability. It will be shown later that the plasma membranes of some cells are impermeable to water.

Before considering the permeability of cells to various substances it is well to discuss the diffusion of substances in general, from one phase to another of a diphasic system in particular.

According to Euler the diffusion of undissociated molecules through water is inversely proportional to the square root of the molecular weight, large molecules therefore diffusing more slowly than small ones. The speed of diffusion of ions is given on page 24 .

Diffusion is retarded by increase in the viscosity of a solution, hence the emulsoids, which enormously increase the viscosity, decrease diffusion. From this it also follows that those substances that increase the viscosity of water diffuse slowly.

The molecules of a substance in solution move in all directions, the substance diffusing from regions of higher to regions of lower concentration. The rate of diffusion is proportional to the concentration gradient.

In the diffusion of a substance from one phase into another, three processes are involved: first, the diffusion to the phase boundary; second, solution in the second phase; third, diffusion away from the phase boundary.

Substances which lower the surface tension collect at the phase boundary, this step in the process being rapid in this case. This concentration in the surface film is due to two factors, 
repulsion by the first phase, attraction by the second phase. According to I. Traube, substances which lower the surface tension of water do so because they have a low molecular attraction for water molecules. Molecules which lower the surface tension of water are repelled by the water molecules and tend to collect at the surface of any other phase in the water. But the degree of this collection is also influenced by the attraction of this second phase for the molecules in question. A substance will not diffuse into a second phase in excess of its solubility unless it is removed by combining with some other substance or changed as by dissociation or association.

After a substance enters the second phase, if it lowers the surface tension of this phase it will tend to remain in the surface film rather than to diffuse into the interior. Its diffusion is also influenced by the viscosity of this second phase.

Thus the rate of diffusion of a substance from one phase to another is determined by a number of factors. The process being reversible, these factors determine the partition at equilibrium.

The partition of a solute between two phases may be determined by shaking up the substance with equal volumes of the two phases, then determining the concentration of the substance in the two phases. A number representing the ratio of these two concentrations is called the partition coefficient. Berthelot and Jungfleisch ( 1872 ) have shown that the partition coefficient of a substance is not affected by the concentration of the substance, provided it has the same molecular condition in the two phases. If, however, the molecular condition changes in one phase with the concentration, the partition coefficient changes with the concentration. For example: consider the partition of common salt between benzol and water. The partition of the undissociated molecules is the same at all concentrations, but salt is dissociated in water and not in benzol. This means that $\mathrm{Na}^{\circ}$ and $\mathrm{Cl}^{\prime}$ are insoluble in benzol. If the salt is very concentrated in the water there will be a large proportion of undissociated molecules and some of these will pass into the benzol, but if the salt is very dilute all, practically, will be dissociated in the water and as the ions are insoluble in benzol all of them will remain in the water. Thus benzol, which dissolves only traces of salt, will take none from an aqueous solution at infinite dilution. 
The plasma membrane is probably a separate phase, and if so, in considering the permeability of a cell we must consider the partition of the solute between the medium and the plasma membrane, as well as between the plasma membrane and the protoplasm. In many cells the protoplasm consists of more than one phase. In the frog's egg, four phase in the cytoplasm and two in the nucleus may be separated by means of the centrifuge (McClendon, I9Io a). The partition of a solute between these phases is therefore to be considered. Some solutes may be in a different molecular condition within the cell, or they may be precipitated or adsorbed to phase boundaries. All of these factors make the subject of cell permeability a very complex one, no general rules without exceptions having been found. All we can do at present is to collect data on the permeability of cells to various substances. The most exact method is the determination of the partition of a substance between water, or some aqueous solution, and the cell, but unfortunately very little of this data has been collected. When a substance is precipitated within a cell, a partition equilibrium may never be reached. If the substance in question is absolutely insoluble in the plasma membrane no diffusion can take place. If the plasma membrane changes with the physiological condition of the cell, substances may become imprisoned in a cell that becomes impermeable to them later. Furthermore, substances may be formed in cells by chemical reactions which will not penetrate cells from the exterior.

Traube attempted to show the ease with which a substance penetrates a cell to be inversely proportional to its molecular attraction to water. Therefore, the more a substance lowers the surface tension of water the more easily it penetrates cells. As was stated above, this is only one of the factors involved. It has been found that substances which very greatly lower the surface tension of water do penetrate cells, but there is not always a constant ratio between the surface tension lowering and the penetration. This same idea championed by Batelli was attacked by Fluzin, who claims that the attraction of the membrane for the solvent is also important. The idea that degree of hydration on the two sides of the membrane influences osmose is also held by $H$. N. Morse.

Overton supposed plasma membranes to be composed of what 
he called lipoids. These are cholesterin, lecithin and some other substances that may be extracted from cells with alcohol, and are soluble in ether. Cholesterin is a polyhydric alcohol, crystallizing in waxy plates. Lecithin is a glyceride in which one hydrogen is substituted by phosphoric acid and the other two by fatty acids, one hydrogen of the phosphoric acid being substituted by choline. Lecithine is a waxy substance which swells in water and alters its power of dissolving other substances, finally passing into a colloidal solution. In order to obtain the lipoids in a dry, fluid condition, Overton and others dissolved them in benzol, xylol, toluol, chloroform or oil of turpentine. Many substances which dissolve in one of these mixtures easily also penetrate cells easily, but exceptions are found. The partition coefficients were usually not determined, and a reliable quantitative method of estimating permeability was not always used.

The composition of the plasma membrane remains a mystery. It seems logical to assume that its building stones are selected from the chief constituents of cells, proteins, fats, lecithin, cholesterin, and carbohydrates. It is a very unstable structure as will be shown later.

Some attempts have been made to find or construct membranes with the same properties as the plasma membrane. Pascucci (1907) impregnated silk with lecithin and cholesterin and found this membrane to show some of the properties of the plasma membrane of the erythrocytes. It was shown by A. Brown (Igog) that the covering of the barley grain has the same permeability as some cells, but he has not proved that this property is due to the cellulose it contains or to any known substance. Beutner claims to have made membranes with some of the properties of the plasma membrane. These will be described in a later chapter. Traube's membranes are easily permeable to acids and bases; halides and nitrates of alkalis, many amines, urea and $\mathrm{H}_{2} \mathrm{O}_{2}$. They are poorly permeable to sulphates, phosphates, carbonates and all salts of alkaline earths and heavy metals, salts of many organic acids, four substituted derivatives of $\mathrm{NH}_{3}$, glycerine, basic and many acid dyes, but impermeable to sugars.

Overton (I899) studied the permeability of plant cells by means of the plasmolytic method, and arranged substances in groups according to their penetration power as follows: 
I) Those substances which penetrate slowest are neutral salts of strong acids and alkalis or alkaline earths, many neutral salts of organic acids or bases, especially acids with short carbon chains, amino acids, and hexoses.

2) Erythrite (4-hydric alcohol).

3) Glycerine (trihydric alcohol), urea and thio-urea.

4) Dihydric alcohols and amides of monovalent acids.

5) Those which penetrate fastest are monohydric alcohols, aldehydes, ketones, aldoximes, ketoximes, and mono, di and tri halogen hydrocarbons, nitriles, nitroalkyles, neutral esters of inorganic and organic acids, and many organic acids and bases, and ammonia, $\mathrm{CO}_{2}$ and the elementary gases. Also free alkaloid bases and some basic dyes.

Increasing numbers of hydroxyl groups decrease the penetrating power, and the same effect results from increasing the number of $\mathrm{NH}_{2}$ groups. Halogen substitution increases the penetrating power. The alkyl or acetyl substitution of the $\mathrm{OH}$ or $\mathrm{NH}_{2}$ groups increases the penetrating power.

As a rule the basic dyes penetrate easily, and the sulphonic acid dyes less easily, but there are exceptions. If the cell is placed in a dilute solution of the dye the minute size of the cell makes it hard to distinguish whether the dye has penetrated or not, since the microscope does not concentrate the color. If the cell is placed in a concentrated solution of the dye and then returned to a colorless medium for observation, the dye that has penetrated may come out again before it can be observed. Many of the basic dyes are precipitated by tannin in the cells so that they may be observed, or they may stain granules or gels. Some dyes that do not at first become more concentrated in the cells than in the medium, become more and more coarsely colloidal within the cells, which constantly absorb more dye in true solution, until they are stained sufficiently to be observed. It is best, in this case, to place the cut stem of the plant in the dye and allow it to remain several days before observation.

Overton's work has not all been confirmed. Janse states that $\mathrm{KNO}_{3}$ penetrates Spirogyra rapidly. Osterhout (1909) claims that calcium and other salts penetrate plant cells at a fair rate. He supposes that Overton may have failed to observe his experiments continuously. 
It is well known that plants concentrate some of the salts of the water that bathes their roots. This is not entirely due to the evaporation of the water from the leaves, since the same is true of water plants. The ash of the kelp, Nereocystis, of the Pacific coast may contain 67.5 per cent $\mathrm{KCl}$. The $\mathrm{KCl}$ in the kelp juice is $0.3^{8} \mathrm{n}$ and in sea water o.or $\mathrm{n}$. The ash of Iridaea edulis contains 23.42 per cent $K=45$ per cent $\mathrm{KCl}$. According ot Stoklasa, however, the ash of Azobacter is almost pure $\mathrm{K}$ salts (laragely phosphates). The mechanism by which the potassium is selectively absorbed is unknown. According to Meigs and Atwood (I9I6) if muscle is placed in pure $\mathrm{KCl}$ solution it absorbs the $\mathrm{KCl}$ faster than the water.

It is certainly true that some neutral salts penetrate some plant cells with exceeding slowness. It was observed by chemical analysis that several grams of yeast absorbed not more than two milligrams of $\mathrm{MgCl}_{2}$ from a molecular solution in five hours (McClendon, I9I2 d). Furthermore, Paine (I9I2 a) used chemical methods and found that yeast absorbs very little if any from more dilute solutions of neutral mineral salts, whereas it absorbs alcohol rapidly. The partition of ethyl alcohol between yeast and water is 0.85 to $I$.

The permeability to injurious substances will be considered under changes in permeability.

The permeability of animal cells is very similar to that of plants. Overton (I902 a) studied the permeability of frog's muscle, concluding that it is essentially the same as that of plants. The relation of muscle to salts is of special interest. Muscle and blood cells store potassium and it is supposed that if they are permeable to salts the potassium will come out and sodium go in, until equilibrium with the blood plasma is reached. Overton claims that neutral salts that do not injure muscle do not penetrate it to any extent. When placed in an isotonic solution of a non-toxic potassium salt, such as the tartrate, the weight of the muscle did not change in fifty hours, but when placed in a toxic isotonic solution, such as $\mathrm{KCl}$, the muscle soon began to swell and died, so that it did not regain its original weight if returned to Ringer's solution. Meigs and Atwood (r9I6) and Siebeck (I913) claim that muscle is permeable to $\mathrm{K}$, but Urano (I908 c) and Fahr (I909) were unable to wash out the potassium from muscle with isotonic sugar solution. 
The permeability of erythrocytes seems to be similar to that of plant cells according to the researches of Grijns (I896), Hedin (1898) and others. Urea, however, seems to form an exceptional solute to which the corpuscles are permeable. Hamburger (I9I6 b) claims that the erythrocytes are permeable to anions and to $\mathrm{Na}^{\circ}$ and $\mathrm{K}^{*}$. .

According to Rona and Michaelis (Igog b) the erythrocytes of dogs and men are permeable to dextrose, judging from the partition of dextrose between corpuscles and plasma (which was not constant, however). On the contrary Kozawa (1914 b) found the erythrocytes of swine, goat, sheep, horse, ox, cat, rabbit and guinea pig very poorly permeable to pentoses, pentites, hexoses, hexites, methylated sugar, disaccharides, amino acids and salts of organic and inorganic acids, and the erythrocytes of men, apes and dogs, the same to all except monosaccharides.

The permeability of other animal cells seems to be similar to that of erythrocytes except in the case of certain cells of the kidney, gut, connective tissue and smooth muscle, which are more permeable. According to Meigs (I9I2 c) smooth muscle cells have no plasma membranes. The composition of other cells in regard to salts is similar to that of the erythrocytes, the $\mathrm{Na}$ content of all the cells is less than that of the blood plasma. Whereas the $\mathrm{K}$ content of the blood plasma is about .026 per cent, that of the heart is .I6I, liver.I7I8, kidney .I643, spleen .I69I, brain .245 , lung .0836 , muscle .3 , and that of erythrocytes .5 per cent.

The convoluted tubule cells of the kidney are permeable to many dyes that do not stain other cells. According to Siebeck (I912) they are more permeable to $\mathrm{KCl}$ than to other salts.

As is well known, phagocytes can take up solid objects, and might include some fluid containing dissolved substances along with them. The "pyrrol" cells of the connective tissue are considered by Evans and Schuleman (I9I4) to take up colloidal dyes "phagocytically" but just what is meant is not clear. The Kupfer cells of the liver also take up these dyes, and J. Voigt (19I4) claims that they take up colloidal silver. The reticulum cells of the spleen and interstitial cells of the testicle behave similarly toward these dyes.

The eggs of some marine (Fundulus), migratory (smelt) and 


\section{OF VITAL PHENOMENA}

fresh water fish (pike, muskalonge) are impermeable to salts and also to water. If Fundulus eggs are taken from the mother or from the sea water in which they are laid, they neither swell nor lose their salts. The fish that develop from these eggs are permeable to water, but there is evidence to indicate that except for the gut and kidneys they are impermeable to salts (Sumner, I905 and 1907, and G. G. Scott, I9I3, $a$ and b). 


\section{CHANGES IN PERMEABILITY OF PLANT CELLS}

That the permeability of a cell may change has long been known. Nägeli ( 1855 ) observed that when plant cells died chlorophyll and other substances diffused out of them. Pfeffer ("Pflanzenphysiologie") explained the movements of plants as due to changes in permeability. It had been shown by Pfeffer and de Vries that the osmotic pressure of many plant cells is very high-when the cells are placed in water, that they do not burst is due to the strength of the cell wall. If water is accessible the plants are firm and elastic as a rubber tube when it is filled with water under pressure. If the water is removed from them by evaporation or by immersion in a concentrated salt solution they wilt and contract. The same is true if the permeability of the cells is increased, as by killing the plant by immersion in hot water or in some toxic solution or gas. But changes in permeability may occur without death of the plant. Such changes follow some external change which we call a stimulus. Plants which respond in this way to stimuli are called sensitive plants. The leaves of Mimosa pudica, and many Leguminoceae and Oxalaceae close after being touched. The stamen hairs of Cynara (French artichoke), Centaurea, Berberis and Helianthemum shorten after an insect walks over them.

Pfeffer observed the shortening of these stamen hairs under the microscope. A fluid exuded from the cells into the intercellular spaces, due to increased permeability of the cells to the osmotic substances which held the water in them. The cell walls had been stretched by an osmotic pressure of about three atmospheres. When this was released the cells decreased in volume and the hair consequently decreased in length. The cells in such cases are not dead because they gradually absorb water and swell (regain their turgor) and the hair lengthens. The osmotic substances that were lost when the permeability increased cannot 
be used to do the work of restoring the turgor, but new osmotic substances must be produced by the cell. According to van Rysselberghe ( 1899 ) some plant cells may increase thelr osmotic pressure by transforming starch into oxalic acid, but his evidence for this is probably slight.

Pfeffer extended these observations to the pulvinus of the sensitive plant leaf. His observations have been confirmed recently by Lepeschkin (I908), who showed that the osmotic pressure of these cells was idecreased when they were stimulated and that not only water, but also dissolved substances, diffused out of them. Waller (Ig04) showed that increased electrical conductivity followed stimulation.

The cells of the sensitive plants are not alone in their changes in permeability. Lepeschkin (I908) and Tröndle (I9IO) found that light increases the permeability of plant cells to salts and in a lesser degree to dextrose. Fluri (I909) observed that the salts of aluminum, yttrium and lanthanum increased the permeability of plant cells to salts and to sugars. After washing out the reagent the cells returned to their normal condition. If Spirogyra were treated with one of these salts, the tannin diffused out of it so that if it were placed in a solution of chinin, no precipitate of this alkaloid and the tannin was formed inside the cells. Fluri interprets these results differently, however, owing to the fact that the viscosity of the protoplasm is increased so that the chromatophores cannot be precipitated with the centrifuge.

De Vries showed that nitric acid increases the permeability of Tradescantia cells to $\mathrm{KNO}_{3}$. In experiments with acids it is necessary to distinguish three conditions: First, the cells may be permeable to the acid, as in case of fatty acids. Second, the permeability of the cell may be reversibly changed by the acid, as in case of mild treatment with fatty acids. Third, the cell may be killed by the acid and its permeability irreversibly increased, as in case of the mineral acids. In this experiment of de Vries the cells were probably killed by the acid.

It was shown by Pfeffer that the anthocyan in plant cells may be used as an indicator to show the penetration of acid. The penetration of acid into the cells of the red beet, red cabbage and red nectar glands of Vicia faba has been observed (Mc- 
Clendon, I9I2 d). The mineral acids penetrate, but the fatty acids more quickly. It is probable that both of these acids increase the permeability.

The same caution should be observed in considering the permeability of cells to bases. E. N. Harvey (I9II) stained Elodea leaves with the indicator, neutral red, and studied the penetration of bases. Ammonia and the amines penetrated more quickly than the alkalis. All of these bases probably increased the permeability.

In the experiments of Czapek (I9IO) the cells were evidently killed and the permeability irreversibly increased. Czapek determined the exit of tannin from Echeveria leaves by the failure of alkaloids to cause a precipitate within the cells. Monovalent alcohols and ketones, ether, urethane, di and tri acetin, sodium oleate, oleic acid, lecithin and cholesterin increased the permeability of the cells to tannin, when the surface tension of their solutions in water sank to about 0.68 . Mineral acids increased the permeability when the concentration just exceeded I/6400 normal, the same concentration at which Kahlenberg and True (18g6) had found the growth of Lupinus to be stopped. Their action is evidently due to the $\mathrm{H}$ ions.

The experiments of Osterhout (I9II) are especially valuable because he was careful that the cells were not killed. He found that the permeability of Spirogyra is increased by pure solutions of $\mathrm{NaCl}$ and that this action is inhibited by the addition of $\mathrm{CaCl}_{2}$. A solution of $\mathrm{NaCl}$ may be hypertonic and yet fail to plasmolyze the cells because it penetrates, but if a little $\mathrm{CaCl}_{2}$ solution be added, plasmolysis occurs even though the osmotic pressure of the salt solution is slightly reduced.

The electric conductivity method was used to determine permeability of animal cells by several investigators (G. N. Stewart, I897, McClendon, I9I0 c). Osterhout (I9I2 a) extended this method to seaweed, finding that the conductivity of leaves of Laminaria (kelp) is increased by immersing them in $\mathrm{NaCl}$, and decreased by immersing them in $\mathrm{CaCl}_{2}$ solutions in a reversible manner. He claims that the size of the cells was not changed by the solutions and since the conductivity of the solutions was the same as that of sea water, the result is not due to a change .in the conduction in the intercellular substance. He concludes, 
therefore, that $\mathrm{Na}$ increases, and $\mathrm{Ca}$ decreases, the permeability of the cells to ions. The intercellular substance in this and many other seaweeds is of large volume in comparison to the volume of the cells. The ash of Laminaria digitata is 22.4 per cent $\mathrm{K}$ and 24 per cent $\mathrm{Na}$. It seems certain that this amount of $\mathrm{Na}$ might be contained in the sea water in the intercellular substance and hence the cells be free from it. Evidently the cells store $\mathrm{K}$ but the mechanism of this storage is unknown.

Osterhout (I9I5 b) observed that salts of $\mathrm{La}, \mathrm{Ce}, \mathrm{Y}, \mathrm{Fe}, \mathrm{Al}$ and $\mathrm{Th}$ first decrease and then increase the conductivity of Laminaria.

The experiments of Osterhout suggest an explanation of the function of salts in relation to plants. It was shown by Nägeli, Pasteur, Raulin (I870) and others that plants require certain salts that apparently do not enter into the composition of carbohydrates, proteins, or fats. Loew showed that these salts are protective rather than nutritive. Thus, he found that either $\mathrm{Ca}$ or $\mathrm{Mg}$ in excess is toxic, but that these two poisons antagonize the toxicity of one another. The experiments of Osterhout indicate that they preserve the normal permeability of the plant cells by their contact with the plasma membrane. To speak more generally, we may say that their presence outside and inside the cells affects the aggregation state of the colloids, and that they affect the plasma membrane, which is probably colloidal.

Potassium is stored in many plants and probably all of it is needed, while on the contrary, the calcium that is stored in plants in the form of insoluble salts seems to be a waste product. Sodium is needed by some marine plants (Osterhout, r912 b). The carbonates, nitrates, phosphates and sulphates are decomposed by the plant in obtaining the carbon, nitrogen, phosphorus and sulphur for the manufacture of proteids and are in this capacity nutritive, but the chlorides are protective. The carbonates and phosphates also serve to preserve the hydrogen ion concentration. Before the leaves fall in the autumn the potassium is absorbed back into the tree, but the calcium is allowed to remain in the leaf. 


\section{CHAPTER X}

\section{NEGATIVE OSMOSE AND THE POLARIZATION OF MEMBRANES IN RELATION TO THE BIO- ELECTRIC PHENOMENA, STIMULATION, ABSORPTION AND SECRETION}

Owing to the wide hiatus yet remaining in our knowledge of this subject, we need especially to guard against definitive statements. It seems best to attempt to explain the unknown in terms of the known, or at least to admit the possibility that the unknown is similar to the known and the invisible of the same structure as the visible.

Of the various conceptions of osmotic membranes, two seem to stand out prominently. The first is that the membrane is a molecule sieve. The fact that the osmotic properties of porcelain membranes increase as the pores become finer, tends to support this view. On this hypothesis, the electric phenomena associated with membranes become capillary electric phenomena. The second conception is that an osmotic membrane is a separate phase that will dissolve some of the constituents of the fluids bathing it. The electric phenomena associated with such membranes are called phase boundary forces by Nernst and Riesenfeld. Since there must be spaces between the molecules of the membrane, this phase becomes a sieve and the difference between the two conceptions seems to be more quantitative than qualitative. It may be asserted that from the first viewpoint we are concerned with the size of pores and molecules, and with adsorption and contact electricity, whereas from the second viewpoint we are concerned with solution, partition coefficient, association and dissociation. But owing to the state of our knowledge of the forces concerned in these phenomena, it is difficult to compare them. Presumably all forces are electric. The dielectric constant of the phase membrane determines its ionizing power and hence its permeability to ions, but the dielectric constant of the 
sieve membrane determines its electric charge, and hence its permeability to ions.

If we try to classify membranes according to their permeability, the boundary between sieves and phase membranes seems to break down. Bigelow and Bartell (1909) showed that the permeability and osmotic properties of porcelain membranes depend on the size of the pores. Whereas the pores in these membranes can be seen, those in gelatine or collodion membranes are invisible. Bechhold (1907) showed, however, that the permeability of collodion or gelatine gels varies directly with the water content, and with high water content large particles could pass through them by filtration. Thus it seems that these emulsoid gels have pores, a fact that may be detected in suspensoid gels with the ultramicroscope. Rubber is also a colloid, at least under certain conditions, although a rubber membrane has been regarded as a continuous phase. Prof. Ira H. Derby tells me that the rate of flow of liquids into a rubber membrane is mathematically the same as the rate of flow into capillary spaces existing in small spherical aggregates, indicating that the liquid passes through capillary spaces in the rubber. This is not only true of native rubber, in which traces of the emulsion structure might supposedly be left, but also of dental rubber, in which the spaces between the original droplets have probably been obliterated by the process of manufacture.

\section{Negative Osmose}

It has long been known that absorption by the gut and secretion by the kidney may take place against osmotic pressure and concentration gradient, and many attempts have been made to rob these phenomena of their peculiarly "vital" attributes. Hamburger (I908) claims that membranes of different composition on the two faces show irreciprocal permeability to water and solutes.

These experiments by Hamburger (which have apparently been discontinued) may be concerned with the phenomenon of negative osmose observed by Dutrochet, Graham, Girard, Bernstein and Bartell. According to Flusin ( 1908 a and b) the negative osmose is due to the fact that the swelling of the membrane is greater on one side than on the other. 
The experiments of Bartell (I9I4) are more illuminating, since he used porcelain membranes that could be studied in a more exact manner. $\mathrm{He}$ had already observed that porcelain with large pores shows no osmotic effects and that the semipermeability varies inversely with the size of the pores, seemingly directly with the molecular weight of the solute. The membranes showing negative osmose are very leaky membranes with pore diameters of about 0.2 microns. No osmotic effects were observed with $\mathrm{MgCl}_{2}$ solutions if the pore diameters exceeded 0.4 microns, negative osmose occurring with pore diameters from 0.4 to o.I microns and positive osmose with pores less than o.I micron in diameter. The fact that the same membrane showed positive osmose with $\mathrm{KCl}$ and negative osmose with $\mathrm{LiCl}$ indicated a relation of ions to osmose, which will be considered in the next section.

Electric Polarization of Membranes and Its Relation to Osmose

The electromotive force (emf) of a concentration cell is due to the diffusion of ions, but since ions of the same kind diffuse in all directions in water, at equal rates, one or two other conditions must also be present. There must either be a membrane that alters the diffusion rate in one direction or there must be an unequal distribution of other ions. Although the molecules of one non-electrolyte may not affect the diffusion of another, ions exert forces on one another through their electric charges. Take, for example, the forced diffusion of ions in a potential gradient. If an electric current is passed through a dilute solution of $\mathrm{HCl}$, the $\mathrm{H}$ ions will carry most of the current because they move nearly five times as fast as the $\mathrm{Cl}$ ions. Now add $\mathrm{KCl}$ until its concentration is twenty-five times as great as the $\mathrm{HCl}$. The $\mathrm{K}$ ions move with the same speed as the $\mathrm{Cl}$ ions, hence they would carry only five times as much current as the $\mathrm{H}$ ions although twenty-five times as numerous. In a pure $\mathrm{HCl}$ solution the $\mathrm{H}$ ions carry $5 / 6$ of the current, whereas in the mixture they carry less than O.I, moving less than O.I as fast as in the pure solution. In this way the addition of $\mathrm{KCl}$ reduces the diffusion poteritial that would be produced at the boundary between solutions of $\mathrm{HCl}$ of different concentrations, the more $\mathrm{KCl}$ present at the boundary, the greater the reduction. To take a simple case, consider the following concentration cell: 
Calomel electrode|. I normal $\mathrm{KCl}$. I n $\mathrm{HCl}|\mathrm{n} \mathrm{HCl}| \mathrm{n} \mathrm{KCl} \mid$ cal. electrode.

The diffusion potential between the two acid solutions may be calculated from Nernst's formula (in millivolts at $25^{\circ}$ ):

$\mathrm{emf}=\frac{\mathrm{u}-\mathrm{v}}{\mathrm{u}+\mathrm{v}} 59 \log \frac{\mathrm{c}_{2}}{\mathrm{c}_{1}}=\frac{3^{\mathrm{I} 8}-65}{3^{18} 8+65} 59 \log \mathrm{IO}=+39 \mathrm{mv}$ on the left. The two diffusion potentials between $\mathrm{HCl}$ and $\mathrm{KCl}$ may be calculated by Planck's formula: emf $=59 \log \frac{\mathfrak{u}_{1}+v_{2}}{\mathfrak{u}_{2}+v_{1}}$ and for the first one is $59 \log \frac{3^{18}+65}{65+65}=59 \log 3.2=+29 \mathrm{mv}$ on the left, and is neutralized by the second which is $+29 \mathrm{mv}$ on the right. Hence the total emf is $+39 \mathrm{mv}$ on the left, but would be less if the two $\mathrm{KCl}$ solutions were of the same concentration. In this concentration cell, the emf is due to the difference in speeds of $\mathrm{H}^{*}$ and $\mathrm{Cl}^{\prime}$. If we increase this difference by interposing between the two $\mathrm{HCl}$ solutions a membrane permeable to $\mathrm{H}^{\circ}$ but impermeable to $\mathrm{Cl}^{\prime}$ the emf will be increased to $59 \mathrm{mv}$, or if the membrane is permeable to $\mathrm{Cl}^{\prime}$ and impermeable to $\mathrm{H}^{\circ}$ the emf will be reversed in direction but still be $59 \mathrm{mv}$. In studying the effect of a membrane on the relative speeds of the two ions of an electrolyte, the use of $\mathrm{KCl}$ is most convenient wherever possible, since the two ions move at the same rate, and danger of unbalanced diffusion potentials at the two ends of the series is eliminated.

The effect of porcelain membranes on the emf of concentration cells was studied by Bartell and Hocker (I9I6). Bartell had observed that negative osmose only occurred if the speed of the anion in free diffusion were greater than the cation, and Hocker found that in negative osmose the speed of the anion through the membrane is greater than the cation. Since the dilute side receives the charge of the diffusing ions, the dilute side is negative in negative osmose and positive in positive osmosis. The porcelain itself is charged negatively except in relatively more concentrated solutions of acids, or the salts of certain polyvalent or heavy or rare metals, which neutralize or reverse the charge and consequently alter the osmose. Appar- 
ently zinc ions cannot reverse the charge of the porcelain. The author observed that if a concentrated solution of zinc sulphate were placed on each side of a porcelain membrane (kindly loaned by Dr. Bartell) and an electric current passed, water was drawn through the membrane to the cathode by electroendosmose. This showed that the water was charged positively, and the water on passing through capillary spaces always takes the opposite charge to the charge of the walls.

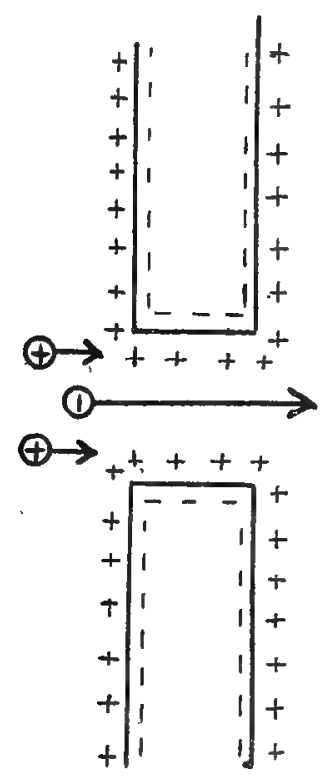

FIG. 27. Scheme showing the mode of diffusion of anions through a large pore in a negatively charged membrane, thus causing negative osmose by electroendosmose.

Bartell and Hocker explain negative osmose as electroendosmose. Since the dilute side becomes charged negatively, it pulls the positively charged water through the membrane, causing the flow to be toward the dilute side.

In Fig. 27 is represented a negatively charged membrane with a large pore. If the pore were small a negative ion could not enter it since it would be repelled by the negative charge of the walls. If cations pass through small pores they tend to make 
the dilute side positive and hence favor positive osmosis by electroendosmose, hence a membrane containing only such pores would show only positive osmosis. Anions can diffuse through the large pore, however, and since they diffuse faster than cations in case of those salts effecting negative osmose, they give the dilute side a negative charge. The water, therefore, that is charged positively (near the walls of the large pore) is pulled through toward the dilute side. We have, then, in favor of negative osmose, the electroendosmose and the water carried by the electrolyte that diffuses toward the dilute side; whereas, against negative osmose, we have the osmotic pressure of the more concentrated solution exerted rather ineffectively against a leaky membrane. It may be added that the explanation given by Freundlich (I9I6) seems at least unnecessary.

It was further observed by Hocker that not only the magnitude but also the direction of osmose is affected by changes in concentration of the electrolyte. Negative osmose did not occur with solutions more dilute than $\mathrm{n} / 5 \mathrm{O}$. Perhaps at this dilution the size of the negative ions is so increased by hydration as to prevent them from passing through the pores. It would be interesting to know whether more dilute solutions show negative osmose with membranes having slightly larger pores.

The experiments of Bethe (I9II) and Bethe and Toropoff (I9I4) may be interpreted as indicating that the charge in the water half of the electric double layer over the porcelain is due, in part at least, to $\mathrm{H}$ ions. They found that when an electric current of more than one volt is passed from non-polarizable electrodes through a $\mathrm{Na}_{2} \mathrm{SO}_{4}$ solution, divided by a diaphragm of gelatine, jelly, collodion, agar-agar, albumin, bladder, gold beaters skin parchment, carbon or clay, the anode side becomes alkaline and the cathode side acid, whereas water passes toward the cathode. Thus water and hydrogen ions pass in the same direction. The water therefore that is charged positively contains an excess of $\mathrm{H}$ ions, which must give it at least part of its positive charge. The side of the membrane toward the anode is alkaline on account of the removal of hydrogen ions. It is impossible for the massing of anions against a non-metallic membrane to cause an acid reaction, since there is no means of robbing them of their charges. 
Bethe and Toropoff found that polyvalent cations tend to reverse the charge of the membrane and, hence, the direction of electroendosmose and disturbance of neutrality, whereas polyvalent anions tend to maintain it. The effectiveness of anions in causing cathodic acidity is as follows: citrate" ${ }^{\prime \prime}>$ phosphate" $>$ oxalate" $>\mathrm{SO}_{4}^{\prime \prime}>\mathrm{I}^{\prime}>\mathrm{Br}^{\prime}>\mathrm{Cl}^{\prime}>\mathrm{NO}_{3}^{\prime}$. The effectiveness of the cations in reversing the charge of the membrane and disturbance of neutrality is as follows: $\mathrm{Co}\left(\mathrm{NH}_{3}\right)_{6}{ }^{\cdots}>$

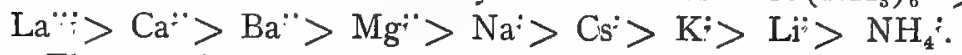

The experiments of Bethe on living cells (I9I6) are, however, open to another interpretation. It was shown (McClendon, I9I4 d) that the anthocyan (or other pigment) in many plant cells is amphoteric. In acid it is red and goes to the cathode, whereas in alkali it is blue or green and goes to the anode, if an electric current is passed through the solution. These changes may be observed in the living cells, which are penetrated easily by acetic acid or ammonia but are easily injured by the reagent or current so that the pigment passes out of them. In successful experiments, all of the pigment is massed in the cathode ends of red cells and in the anode ends of blue cells. When the plant is fresh the pigment is usually reddish, but contains a small proportion of blue, and sometimes violet cells may be found. If an electric current is carefully passed through a violet or redviolet cell under the microscope, the red portion of the pigment will mass in the cathode end of the cell, and the blue portion in the anode end. This phenomenon may have led Bethe to suppose that the passage of the current through the membrane disturbed the reaction. His experiments on cells stained with neutral red are not, however, open to this objection. It should be noted that the anode end of the cell is the cathode side of the membrane against which the pigment is massed.

It might be supposed that the colloidal membranes used by Bethe and Toropoff are so different from the porcelain used by Bartell that the results may not be compared. Bethe and Toropoff found, however, that membranes of clay or carbon behaved in the same way as gelatine membranes, in the disturbance of neutrality on the passage of an electric current through them. We may, therefore, safely use the foregoing findings in explaining the results obtained with various membranes. Flusin 
(Igo8) observed that a solution of tartaric acid passes through pig's bladder into pure water (negative osmose). Evidently the acid reversed the charge on the membrane, making it positive, the greater speed of the positive ion causing the negative osmose. Although Flusin adopts another explanation for anomalous osmose, Girard (1908-I3) shows conclusively that electroendosmose is at least in part the correct explanation. If the solutions on the two sides of the membrane are isotonic the driving force of osmose is the diffusion potential causing electroendosmose. In the following table the first column gives the solutes used in the isotonic solutions, the second the charge of the membrane, the third column the direction of the emf (positive current), the fourth the direction, and the fifth the relative magnitude of the osmose.

Isotonic membrane diffusion direction of magnitude of solutions potential emf osmose osmose

Sucrose

Tartaric ac.

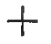

$\uparrow$

$\downarrow$

$\uparrow$

$\downarrow$

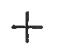

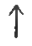

$\downarrow$

$\downarrow$

$\downarrow$
33

IO

30

20

Oxalic ac.

$\mathrm{Na}_{2} \mathrm{SO}_{4}$

With a membrane separating isotonic solutions of $\mathrm{NaCl}$ and $\mathrm{KCl}$ there was no emf and no osmose. The same was true of $\mathrm{KCl}$ and $\mathrm{Na}_{2} \mathrm{SO}_{4}$. Although these experiments of Girard antedate those of Bartell and Hocker, the latter have been described first on account of the simple character of the membranes and the remarkable relation of the diameter of the pores to their properties. Although it may not have been shown that all anomalies in osmose are due to electroendosmose, it seems unnecessary at present to accept the hypothesis of Flusin that osmose takes place through the membrane from the side of least swelling (smaller pores) to the side of greatest swelling (largest pores). At any rate, the size of the pores in porcelain membranes is not affected by the character of the solute (Hofmeister series). 
Bernstein ("Elektrobiologie," p. I62) supposes electroendosmose to be the explanation of his observations on negative osmose through copper ferrocyanide membranes, although he records no observations on the electric charge of the membranes or the emf. On filling the osmometer with $\mathrm{K}_{4} \mathrm{FeCy}_{6}$ solution and immersing it in $\mathrm{CuSO}_{4}$ solution of slightly higher osmotic pressure (calculated from the freezing point) he observed a rise in the manometer, in some cases overflowing at $34 \mathrm{~cm}$. In order to eliminate any possibility of error from the unequal temperature coefficients for the osmotic pressures of the two solutions, he repeated the experiments at $0^{\circ}$, and yet the manometer overflowed. The emf of these membranes as measured by Brünings are given below.

Comparison of the Polarization of Various Types of Membranes

The behavior, mentioned above, of the copper ferrocyanide membrane may seem surprising, because, of all dead membranes that have been investigated, it has proved to be the most truly semipermeable in osmotic experiments. Brünings (I907) using isotonic solutions of $\mathrm{CuSO}_{4}$ and $\mathrm{K}_{4} \mathrm{FeCy}_{8}$ observed that the $\mathrm{CuSO}_{4}$ side is electropositive. He supposed the emf to be due to the permeability of the membrane to $\mathrm{K}$ ions, and its being impermeable to the other ions. If this were the case, the emf should be proportional to the logarithm of the concentration of $K$ ions (which is not true, as the emf remained very near $100 \mathrm{mv}$ with large variations of $\mathrm{K}$ ion concentration). It has been shown by H. N. Morse (I9I4) that these membranes rapidly deteriorate when in contact with other ions than those from which they are formed, and for this reason it may be impossible to decide this question. When we remember, however, that Morse observed that the membrane may be greatly thickened by the passage of an electric current, it becomes clear that the membrane cannot be absolutely impermeable to copper and ferrocyanide ions until its electric conductivity becomes practically zero. Evidently, during the formation of the membrane (without the aid of the current) negative charges are removed from the ferrocyanide solution and positive charges are removed from the copper solution, which would produce an emf opposite to that found by Brünings if the membrane is impermeable to all ions except copper and ferrocyanide ions. 
According to Beutner (I9I3, b) copper ferrocyanide membranes are permeable only to the cations of many salts. In order to prevent deterioration of the membrane he set up this series: Calomel electrode $\left|\mathrm{K}_{4} \mathrm{FeCy}_{6}\right| \mathrm{CuSO}_{4}+\mathrm{x}$ mol. salt $\mid$ calomel electrode. With $\mathrm{n} / 40 \mathrm{NaCl}$ in the $\mathrm{CuSO}_{4}$ solution the emf was 29 millivolts, and with $\mathrm{n} / 400 \mathrm{NaCl}$ in the $\mathrm{CuSO}_{4}$ solution the emf was $80 \mathrm{mv}$, or a difference of $5 \mathrm{I} \mathrm{mv}$ produced by diluting the salt ten times, whereas the difference calculated by Nernst's formula is $58 \mathrm{mv}$.

Taking Beutner's results as evidence that these membranes are permeable only to the cations of the salts of alkali metals, we may explain the negative osmose observed by Bernstein on the assumption that the membrane is negative, hence the water positive, the diffusion of the $\mathrm{K}$ ions leaving the ferrocyanide solution negative.

One of the clearest demonstrations of the relation of permeability to membrane polarization was shown by Bayliss (I9II). He separated two solutions of the sodium salt, congo red, by a membrane of parchment paper. The $\mathrm{Na}$ ions can easily pass through the membrane but the congo red anions cannot pass through a dense grade of the paper. Therefore the dilute side is electropositive. Bayliss found the emf, when measured immediately after the membrane was wet with the solutions, to approximate that calculated by Nernst's formula, but to fall gradually. He explained the fall by assuming that the osmotic pressure of the concentrated solution draws water through the membrane, thus forming a dilute layer next to the membrane and lowering the difference in concentration between the solutions in immediate contact with the two faces of the membrane.

The author found that even with $\mathrm{KCl}$ solutions, parchment paper gives an emf, but the potential difference falls very rapidly, so that it is impossible to measure the initial potential with the potentiometer method used. The fall in potential is not entirely due to dilution of the more concentrated solution, since after removing and partially drying the membrane and renewing the solutions, the original potential is not obtained. Since the emf becomes zero after a comparatively short time, the fall is probably due, at least in part, to swelling of the membrane and consequent enlargement of the pores. In one experiment an emf of 
$40 \mathrm{mv}$ was observed. With a wooden membrane and $\mathrm{KCl}$ the emf was $7 \mathrm{mv}$, and the dilute side positive.

The emf produced by porcelain membranes in Hocker's experiments was very small, due to the fact that he waited twentyfour hours for equilibrium to occur before making the reading. He found that time to be required owing to the thickness of the porcelain. Very irregular results were obtained if the reading were taken soon after filling the apparatus. The author observed that the bowl of a clay pipe showed negative osmose and was thin enough to come to an equilibrium more quickly, provided the previous solution had been thoroughly soaked out of it. Brünings (op. cit.) found Niernst's formula to be approximated with a burned clay membrane separating $n / 100$ and $n / 1000$ $\mathrm{NaCl}$ solutions, the observed emf being $50 \mathrm{mv}$ and the calculated $59 \mathrm{mv}$. Brünings obtained rapid equilibrium by soaking the membrane in the more concentrated solution, in one case boiling it in this solution sixteen days with a reflux condenser. Brünings tried membranes of burned clay, wood, bone, carbon and marble with $\mathrm{NaCl}$ and $\mathrm{KCl}$, and in every case the dilute side was positive. Since the $\mathrm{Cl}$ ion is faster than the $\mathrm{Na}$ or $\mathrm{K}$ ion these membranes must be more permeable to cations than to anions. Probably this is due to repulsion of the anions by the negative charge of the membrane.

The effect of the electric charge of the membrane on its relative permeability to anions and cations is illustrated by an experiment of Mines' (I9II b). In the following concentration cell the emf is zero:

\section{$\mathrm{Zn}, \mathrm{ZnSO}_{4}|\mathrm{n} / 8 \mathrm{NaCl}| \mathrm{n} / 8 \mathrm{o} \mathrm{NaCl}|\mathrm{n} / 8 \mathrm{NaCl}| \mathrm{ZnSO}_{4}, \mathrm{Zn}$.}

If, however, a gelatine membrane is placed between two of the $\mathrm{NaCl}$ solutions the dilute side becomes positive with an emf of about $60 \mathrm{mv}$. The gelatine, therefore, is more permeable to cations than to anions, and it is well known that natural gelatine is electronegative. Gelatine is made positive by the ions of polyvalent metals, and hence Mines treated the gelatine with a salt of Gadolinium to test its effect on the emf. The same gelatine membrane used above, after treatment with $\mathrm{GdCl}_{3}$ and being returned to the concentration cell, caused an emf of $8 \mathrm{mv}$, but the dilute side was negative, showing that the membrane had 
become more permeable to anions or less permeable to cations - perhaps both.

Selective permeability of membranes to ions has often been noticed in electrical transference experiments. Hittorff separated the solution between the electrodes into segments by means of ox gut membranes, in order to prevent backward diffusion or convection currents. He supposed the membrane did not affect the results (transference numbers), but it is now known that the effect is small in some cases, very large in others. Bein (1899) found that earthenware membranes were the safest to use. Fish bladder membranes caused but small errors with neutral salts of alkali metals, but large errors with $\mathrm{CuSO}_{4}$ solutions. The $\mathrm{SO}_{4}$ ions passed the membrane, but the $\mathrm{Cu}$ ions combined with the substance of the membrane, and hence were held back.

The action of gelatine membranes in holding back $\mathrm{H}$ ions in a similar manner has been noticed by Girard and by Cybulski (I903) and Cybulski and Dunin-Borokowski (1909).

Where the membrane clearly forms a separate phase the electric phenomena have been called by Nernst and Riesenfeld (I902) phase boundary forces. If the speed of the ions in two phases is different, the addition of the electrolyte to either phase will cause a difference of potential at the phase boundary, which is different from that which occurs in a similar concentration gradient in either phase alone. The limiting case is that in which the speed of either ion becomes zero (in the membrane). Such is the case with a metallic membrane, which may be considered impermeable to any ion except the cation of the same metal. Although the atom and its electron part company in passing through the membrane, and the ion as such does not pass through, the effect is the same, since an ion is given off from one side at the instant that an identical ion is deposited on the opposite side of the membrane. It seems proper, therefore, to speak of the permeability of a metallic membrane to an ion, when the mechanism of the passage of the ion or its components through the membrane is of no significance to the point of discussion. When an electrolyte dissociates into two ions to only one of which the membrane is permeable, the emf may be calculated by Nernst's formula for electrode potentials. The emf is $59 \mathrm{mv}$ 
at $25^{\circ}$, when this ion is monovalent and is ten times as concentrated on one side of the membrane than on the other.

It is characteristic of most of the separate phase membranes that have so far been studied that they are very poorly permeable to water. Their ionizing power is often very small, and excepting the metallic membranes, they are poor electric conductors. Hence the measurement of the emf with a galvanometer, capillary electrometer, or even a potentiometer, is sometimes impossible. A quadrant electrometer of Lord Kelvin's or Dolezalek's type is usually used.

For the general case of a membrane permeable to both ions of the solute, Nernst's ideal formula in millivolts at $25^{\circ}$ is,

$$
\mathrm{emf}=59\left(\frac{\mathrm{u}-\mathrm{v}}{\mathrm{u}+\mathrm{v}}-\frac{\mathrm{u}^{\prime}-\mathrm{v}^{\prime}}{\mathrm{u}^{\prime}+\mathrm{v}^{\prime}}\right) \log \frac{\mathrm{c}_{1}}{\mathrm{c}_{2}}
$$

where $u$ and $v$ are the speeds of cation and anion in water and $u^{\prime}$ and $v^{\prime}$ in the membrane. Though the emf so calculated would be correct if the two aqueous solutions were connected so as to form a closed circuit, the emf could not be measured under such conditions. If calomel electrodes are used, the solutions connecting this concentration chain to the electrodes would have an effect on the total emf as measured.

Examples of membranes permeable to only one of the two ions of an electrolyte are given by Cremer (Ig06) and Haber and Klemensiewicz (I909). Membranes of ice, benzol, toluol, metaxylol, nitrophenol, and thin, water soaked glass, acted as. though they were permeable only to $\mathrm{H}$ ions. When the $\mathrm{H}$ ion concentration on one side of the membrane was ten times as great as on the other side, the average emf with soft glass was $52 \mathrm{mv}$ and with hard glass $59 \mathrm{mv}$, whereas that calculated from Nernst's formula is $58 \mathrm{mv}$ at $20^{\circ}$. The emf with the other membranes was almost as great. Haber and Klemensiewicz used almost pure solutions of acids and bases (without buffers) and, in their tables, did not correct for dissociation. Furthermore, they made no accurate measurements near the neutral point. Since the reaction of all living cells or fluids bathing them is near the neutral point, it seemed worth while to make such determinations, in order to ascertain whether such membranes 
might account for the bioelectric phenomena. Prof. A. F. Kovarik kindly took the readings with an improved Dolezalek's electrometer. Benzol was used as a membrane. Its conductivity is so low that the concentration cell had to be enclosed in a metal cage before constant results were obtained. The $\mathrm{H}$ ion solutions were $\mathrm{PH}=6$ and 7 made with the aid of Sörensen's phosphate mixtures and tested with the hydrogen electrode. The emf was 3I mv, whereas the theoretical is $59 \mathrm{mv}$. This is not, however, a greater discrepancy than in some of Haber and Klemensiewicz's experiments with benzol, the potential difference between $n /$ ro and $\mathrm{n} / \mathrm{roO} \mathrm{HCl}$ being only $\mathrm{I} 8 \mathrm{mv}$. The emf of living muscle as measured, is not usually greater than $3^{\mathrm{I}} \mathrm{mv}$, and since the $\mathrm{PH}$ of the blood is about 7.5 the reaction of the muscle contents need only be $\mathrm{PH}=6.5$.

Whereas, in the above membranes, their chemical nature seemed unimportant, ice, glass and carbocyclic compounds behaving in the same manner, the permeability of certain other membranes depends, according to Beutner ( $1913 \mathrm{~d}$ ), on a chemical reaction. Beutner used one series of membranes permeable only to cations and another only to anions. An example of the first class is salicylic aldehyde, that on oxidation formed traces of salicylic acid. If such a membrane separates solutions of the same salt of different concentrations, the dilute side becomes negative. If both solutions are very dilute, Nernst's formula may be used to calculate the emf approximately. According to Beutner the cations pass through the membrane by being transformed into salicylates, which are soluble in salicylic aldehyde. Beutner's experiments were repeated with $n / 100$ and $n / 1000$ $\mathrm{KCl}$ on the two sides of the membrane, and an emf $=36 \mathrm{mv}$ obtained on Prof. Kovarik's electrometer.

The conductivity of the salicylic membrane is too low to use the potentiometer if the ordinary U-tube is used in making the concentration cell, but at least qualitative results were obtained in the following manner: A solution of $\mathrm{CaCl}_{2}$ of greater specific gravity than the aldehyde was placed in the bottom of a beaker and a glass tube introduced into it. A layer of salicylic aldehyde (to which some salicylic acid had been added to increase its conductivity) was floated on top of the $\mathrm{CaCl}_{2}$ solution, and a dilute solution of $\mathrm{CaCl}_{2}$ added as a third story. In this way 
a membrane of larger surface and less thickness was made. On connecting one calomel electrode with the dilute solution and the other through the glass tube with the concentrated solution, an emf of $25 \mathrm{mv}$ was measured with the potentiometer, the dilute side being positive.

The other type of membrane is an organic base, which, according to Beutner, combines with the anion of the electrolyte to form a salt soluble in the membrane. His experiment was repeated with o-toluidin, in the same manner as with salicylic aldehyde, and obtained $27 \mathrm{mv}$ with the Dolezalek electrometer. In order to use the potentiometer, chloroform was added to the toluidin to make it heavier, and $80 \mathrm{mv}$ obtained, the dilute side being negative.

In order to obtain larger membranes, parchment paper tubes were soaked in salicylic aldehyde, toluidin or anilin. In each case the emf was what would be expected with parchment paper alone, but was maintained for a longer time. For this reason it seems possible that the organic liquid was effective in retarding the wetting of the paper with consequent enlargement of its pores. If this is true it shows a method of finding the initial emf with parchment paper. Although with toluidin alone the dilute side is negative, when it is applied to parchment paper the dilute side is positive. The emf with $n / 100$ and $n / 1000 \mathrm{KCl}$ was $59 \mathrm{mv}$, and substituting toluidin, was $52 \mathrm{mv}$.

According to Loeb and Beutner (I9I3) a solution of lecithin in $\mathrm{m}$-cresol acts in the same way as salicyl aldehyde when used as a membrane in a concentration cell. But since higher fatty acids could be substituted for lecithin, it seems probable that fatty, or glycerophosphoric acid, formed by the decomposition of lecithin was the active agent.

\section{Bioelectric Phenomena}

The electromotive forces produced by living matter have always been a subject of interest, if not amazement. The shock of some electric fish is several hundred volts, and may be felt while the fish is entirely submerged in sea water. On the other hand, the ordinary electric potential differences observed in living matter never reach $0 . I$ volt. The secret of the electric organ lies in the connection of the elements in series. Brünings ob- 
served that if one frog's skin is laid on another, the emf is doubled. In the same way he found the emf of two concentration cells with porcelain membranes to be doubled when they were connected in series. The principle is the same as that of the voltaic pile, and only the nature of the membrane is different. A dissection of an electric organ reveals the serial arrangement, and also (in all electric organs related to muscle tissue) that the side of each element on which the nerve enters is electronegative (Pacini). It seems probable that the manner of production of electricity is the same in the electric organ as in muscle or nerve or any sensitive cell. We shall therefore proceed to discuss the general question of the exhibition of electromotive force by the cell.

The electromotive force of a single cell was observed by Hyde (1904). She placed one non-polarizable electrode on the animal pole and one on the vegetative pole of the egg cell of a fish. When the cell began to divide the animal pole became negative.

Owing to the small size of most cells, it is usually more convenient to study these phenomena in tissues in which similar cells are arranged in multiple. The frog's muscle is convenient. It is possible to measure the difference in potential between the inside and outside of the cells by cutting off one end of the muscle and leading off from the cut surface (interior) and the intact surface (exterior). The interior is found to be negative in comparison to the exterior, which causes an electric current to flow through any external conductor that may be present from the intact to the cut surface. This current is often called the current of injury, without any proof that the injury, as such, has anything to do with it, but the expression is convenient.

Wilhelm Ostwald suggested that the muscle is a concentration cell with a membrane, and Bernstein developed the details of this idea. No one doubts that the muscle is a concentration cell, but the opponents of Bernstein's hypothesis doubt that a membrane has anything to do with it, since concentration cells may be made without membranes. But the emf of the current of injury of a fresh muscle is from 40 to $80 \mathrm{mv}$ and no concentration cell without a membrane has been known to give such a high emf unless it contained strong acids or alkalis, which are incompatible with life. There seems to be no valid evidence against, and much for, 
Bernstein's theory. Furthermore, it is the only theory that will account for all of the facts.

According to Bernstein, the striated muscle fiber or cell is surrounded by a membrane or surface (called the plasma membrane since it is the superficial layer of the protoplasm), which is more permeable to the cation than to the anion of some electrolyte more concentrated in the interior than on the exterior of the muscle cell. The cations passing through the plasma membrane leave the interior negative, and form a positive outer stratum of an electric double layer thus formed at the surface of the cell. Many attempts to determine the electrolyte have been indecisive, but it seems probable that the cation in question is the hydrogen ion, in which case the cell surface must be relatively impermeable to all anions. This assumption has the advantage that it avoids the necessity of difference in osmotic pressure on the two sides of the membrane. A diagram of this

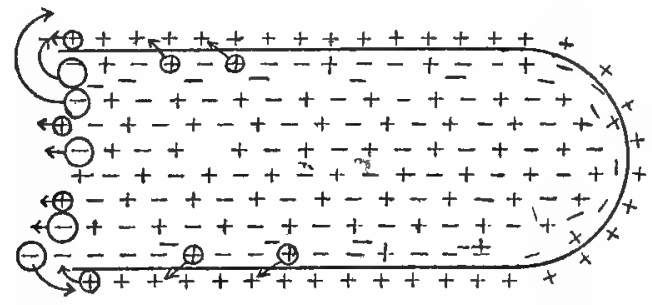

FIG. 28. Scheme showing the plasma membrane of a cell torn open at one end and liberating the excess of anions. The + and - indicate the charge of ions and the arrows the direction of diffusion. Only + ions can come out through the intact plasma membrane and hence there is an excess of - ions on the interior. The tear causes a negative charge to appear at the surface and give rise to the current of injury.

idea is shown in Fig. 28, representing a muscle cell with one end cut off to produce the current of injury. The $(-)$ signs are anions that can escape only at the cut end. The current of injury is caused by the escape of the anions at the cut surface. What these anions may be is immaterial, but presumably they include proteids with negative charges and anions of carbonic and lactic acids produced in the muscle fiber.

The acidity within the cell necessary to produce such an emf as in muscle need be only that of a molecular solution of $\mathrm{CO}_{2}$. 
The $\mathrm{H}$ ion concentration of the blood at $20^{\circ}$ is about $\mathrm{PH}=7.5$ and with such a reaction outside the cell and $\mathrm{PH}=6.5$ inside the cell, the theoretical current of injury would be 58 millivolts, which is about the average value actually found. With the model membrane given in the first section, the emf did not reach this theoretical value, but it is reached in the average of one series of determinations by Haber and Klemensiewicz.

Not only is the cut surface of a muscle negative, but the region of a muscle that is stimulated becomes negative for a fraction of a second. The current produced by this negativity is called the action current, and since the current of injury is produced by the removal of the resistance to diffusion at the cut surface, the action current is probably due to a momentary increase in permeability at the stimulated surface. If this is true we should be able to increase the electric conductivity of muscle momentarily, by stimulating all of the fibers simultaneously. Since it is not easy to measure conductivity in a few thousandths of a second, even with a string galvanometer on stimulating the nerve, another method of stimulation was adopted (McClendon, I9I2 c). The conductivity of a resting muscle was measured by passing an alternating current transversely through it. By putting in resistance this current was made too weak to stimulate the muscle. After the conductivity was determined, the resistance was short circuited and the conductivity determined while the muscle was thrown into tetanus by the alternating current, and found increased from 6 to 28 per cent. It has been objected that the action current of the muscle reinforced the alternating current, but the contrary is true. The muscle is stimulated on the side of the momentary cathode, and the stimulated region, becoming negative, opposes a cathode to a cathode, hence opposes the current. It is also stated that the change in form of the muscle fibres increases the cross section of the interfibrillar spaces. This objection seems to have no theoretical basis. If a vessel is filled with small spheres the cross section of the space between the spheres is the same as though it were filled with large spheres. In my experiments a relatively large muscle was pressed between two platinized platinum discs as electrodes. Between the discs were a large number of cylinders (fibers) and when the muscle 
contracted it was drawn in at the ends and pushed out at the sides, so that the same area lay between the electrodes, but was now composed of a smaller number of larger cylinders. It may be objected that the surface of the contracted fibers is thrown into waves but this does not affect the problem, because such fibers can be resolved into an infinite number of short cylinders of different sizes placed one on another. If a change in the diameter of all of the fibers does not affect the conductivity, a local variation in the diameter of one fiber does not affect it. It seems that the evidence points to an increase in the permeability of the muscle on stimulation.

It was shown by Kunkel (I887) and Burdon-Sanderson ( I888) that an action current follows stimulation of the sensitive plant, Dionea, and the same has been found true of a host of plants by A. D. Waller. It seems probable that an action current (blaze current) follows stimulation of any plant except perhaps most marine plants, as indicated by Waller.

This increase in permeability may cause movements (in addition to electric phenomena). Pfeffer ("Physiol. Untersuchungen" I and 2, I873) studied the movements of plants. The stiffness of a plant is due to the pressure or turgor within its cells, and Pfeffer observed that plant movements are caused by local variations of turgor. Each cell is surrounded by a semipermeable plasma membrane. The osmotic pressure within the cell causes the absorption of water, from the capillary spaces between the cells. When the plant is stimulated certain cells lose their semipermeability and the cell sap filters out into the intercellular spaces, causing a local shrinkage of the tissue.

Waller (I904) observed that the conductivity of plants may increase roo fold (or less) on stimulation, thus giving more evidence for permeability increase. He observed the blaze current to last fifteen minutes in some cases, which makes it easier to study conductivity during the stimulated state.

Whereas the action of the sensitive plant may reach a maximum one second after stimulation, movement does not begin until two and one-half seconds after stimulation. The time required for diffusion together with the mechanical inertia of the parts probably accounts for the delay.

We may sum up the data on the universality of the bioelectric 
phenomena by saying that the current of injury has been observed in all living tissue where it has been looked for, but that action currents have been observed in the leaves of land and fresh water plants, and in animal nerve (and retina), muscle and glands and electric organs derived from them, as well as in the eggs of fish. These electric phenomena are due to changes in permeability to ions. The excited state (increased permeability) remains but a few thotusandths of a second in muscle or nerve but may remain fifteen minutes in plants.

The most wonderful thing about the electrical variation of muscle, nerve or sensitive plant is that it is self propagated. The results of study of electrical stimulation have made the self propagation of the electrical variation a necessary consequence of excitability. It is probably the negative variation of nerve that stimulates another nerve or the motor end plate of the muscle. The medullated nerve fiber is surrounded by an insulating sheath, broken only at the occasional nodes of Ranvier. When a minimal current is sent through a medullated nerve crosswise no stimulation takes place, apparently because of the insulation of the fibers. But when it is sent lengthwise through the nerve it may enter at one node and leave the fiber at another. (The author found non-medullated nerves of Limulus to be as sensitive to a current sent crosswise as to a longitudinal current.) The nerve is stimulated nearest the cathode or apparently where the current leaves the fibers at the nodes nearest the cathode. In other words, the approach of a cathode to the plasma membrane stimulates the nerve fiber. But the stimulated region becomes electronegative and hence a little cathode. It stimulates regions adjacent to it, and the stimulated area spreads like a fire. The reason for this can only be learned when we discover the cause of the increase in permeability of the plasma membrane. $H$. N. Morse (I9I4) states that copper ferrocyanide membranes are colloidal and that a crystallization, resulting in increase of the size of the particles, increases the permeability of the membrane. But the size of colloidal particles may be considerably increased without crystallization (by electrical disturbances). Perhaps the plasma membrane is colloidal and the sudden electrical disturbance, due to the approach of a cathode, causes the particles to aggregate into groups. If the plasma membrane is composed of 
negative colloids like the rest of the cell, the approach of a cathode would tend to lessen the charge on the particles and induce aggregation. The negative charge of cell colloids is illustrated by the author's experiments in passing a constant current through plant (McClendon, I9Io, b) and animal (McClendon, I9I4, d) cells. Chromosomes, yolk granules, and protoplasm in general pass toward the anode, whereas water passes toward the cathode and forms a blister on some animal cells. If the plasma membrane is composed of the same colloids as the cell interior it is probably negative. It may be objected that these colloids become positive in an acid medium and that this negativity is incompatible with the assumed acidity of cell interiors. But the isoelectric point of most of these colloids is much farther on the acid side than the reaction we have assumed for the interior. Furthermore, the plasma membrane is nearer the blood which is alkaline, and is probably bathed by an alkaline tissue juice. The cell sap in the cells of red flowers has an acid reaction.

The rate of propagation of the excitatory impulse may be I $\mathrm{mm}$ per second in some plants and 2000 in mammals. It is probably very slow over the bodies of nerve cells and motor end plates, as there is some delay in conduction through these regions. Mayer (1916) found that nerve conduction varies almost as the concentration of electrolytes around the nerve. Lillie (I9I6 b) supposed nerve conduction to vary with electric conductivity but Mayer has not observed an exact parallel.

Since glands are the seat of an emf, and the skin contains glands, the skin is the seat of an emf which varies with glandular activity. If a constant electric current is passed through the body, the electrical resistance is found to be high. If a galvanometer is interposed in the circuit, the needle may soon come to a constant deflection, but if the subject becomes mentally excited, as on hearing bad news, the galvanometer deflection is suddenly increased. This is called the psychogalvanic reflex, and is used by clinicians and psychologists in the examination of the reactivity of persons. Gildemeister (I9I5) in a very elaborate and carefully controlled series of experiments, showed that the psychogalvanic reflex is due to increased permeability of the sweat glands, which have been stimulated through sympathetic nerves. 
The emf of the frog's skin has been the object of many investigations, but the results so far are for the most part confusing. Schwartz (19I5) found that the electrical conductivity of the frog's skin is increased when the nerves to the skin glands are stimulated. It is probable that the emf of the frog's skin. is analogous to the action current, or more nearly to the current of injury of muscle, and is due to the permeability of one side of the gland cell and semipermeability of the other, the latter being the seat of the emf. The experiments of Bayliss (I908) tend to support this view. On passing an alternating current through the frog's skin, he observed that it acted to a certain extent as an electric rectifier, allowing a current to pass more easily in one direction than in the other. The same effect was produced by a parchment paper membrane separating a solution of congo red from water. This dye dissociates into $\mathrm{Na}$ ions and enormous anions. If the cathode is placed in the water, the current is carried through the membrane by the $\mathrm{Na}$ ions, but if the anode is placed in the water, the anions attempt to pass through the membrane to the anode, but are unable to do so.

Perhaps much of the confusion arising from the study of the emf of living tissues is due to the fact that they are made of more than one kind of cell, and that the permeability of the cells may be changed by the experimental procedure. Consider, for example, the excised muscle. If it is bathed on all sides by lymph or blood, any emf observed is probably due to the muscle cells, but if one electrode is connected to it by a more concentrated solution and the other by a dilute solution of electrolytes, the system may form a concentration cell in which the perimysium or muscle sheath is the chief membrane concerned. The fact that porcelain membranes may act in this capacity, shows that relatively permeable membranes must be taken into consideration, even membranes allowing an appreciable amount of filtration. Oker-Blom (I9OI) studied what he called the contact potential between muscle and certain solutions. This work was extended by Brünings (1907) who attempted to prevent the injurious effect of diluting the salt solutions by the addition of sugar to make them isotonic. He found the contact potential between frog's muscle and sugar solution to be 50 millivolts, with the sugar side positive, and the same result was obtained with 
dead muscle. Perhaps the perimysium surrounding the muscle, being formed of electronegative colloids, is more permeable to the cations than to the anions of the electrolytes between the muscle fibers, hence the dilute side (in reference to the salt) is positive. When the end of a muscle is dipped in water, the dilute (water) side is positive until the water injures the muscle, when the dilute side becomes negative, the perimysium emf being superseded by the current of injury. Brünings found that when the entire body of a man, a frog or a plant is used as a membrane in a concentration cell, the dilute side is positive.

Loeb and Beutner (I914), who extended Brünings's experiments, oppose all hypotheses on the origin of animal electricity which relate to membrane destruction or selective permeability to ions. They admit, however, that a current of injury is produced by cutting off part of the skin of an apple, and that the same increase in permeability may be produced by merely pressing the skin with the finger. The argument they bring against Bernstein's hypothesis is the similarity between living tissue on the one hand and Beutner's acid membranes or lecithin in guaicol on the other, when used as membranes in certain concentration cells. It seems perhaps more significant that in a previous paper (I912) they interpret their results as showing that the seat of the current of injury is the intact membrane and that it is caused by the increase in permeability or removal of the membrane at the injury.

Having thus sketched the membrane hypothesis, it may be advisable to add additional proofs of its validity. If the membrane theory is correct, change of temperature of the cut end of a muscle should not affect the current of injury since the cut end is not the seat of the emf, but the current of injury should be proportional to the temperature of the intact end. From Nernst's formula emf (in millivolts) $=.198 \mathrm{~T} \log \frac{\mathrm{c}_{1}}{\mathrm{c}_{2}}$, where $\mathrm{T}$ is the absolute temperature, hence any change in $\mathrm{T}$ would cause a corresponding change in the emf. Bernstein ("Elektrobiologie," p. 97) found this to be the case, at least between the temperatures $18^{\circ}-0^{\circ}$. In case two points on an intact muscle are at different temperatures Nernst's formula becomes emf $=.{ }_{.19} 8 \mathrm{~T}_{1}$ 
-.I98 $\mathrm{T}_{2}$, which means that a thermocurrent will be produced proportional to the difference in absolute temperature. Bernstein found this to be true also, the warmer end being positive.

Further proof of functional change in permeability of the plasma membrane to electrolytes was found on measuring the conductivity of sea urchin eggs by the Kohlrattsch method. The conductivity increases when the eggs are stimulated to begin development (McClendon, I9ıо $c, e$ ). This was confirmed by Gray (I9I3, a, b) who observed an increased conductivity in the first fifteen minutes of development. Just what electrolytes were affected by this increase in permeability was not ascertained, but in case of the frog's egg, the author was able to show an increased permeability during the first hours of development to $\mathrm{Na}, \mathrm{K}, \mathrm{Mg}, \mathrm{Ca}$, and $\mathrm{Cl}$ (I9I4 a, rgr $5 \mathrm{a}$ ). The eggs were caused to develop in distilled water by means of electric stimulation, and the salts which diffused out analyzed and compared with those diffusing out of unstimulated controls.

A change in permeability may also accompany pathological changes. It was shown (McClendon, I9I3 a, I9I4 c) that the eggs of certain fish which are impermeable both to water and to salts, may be made permeable to salts by various toxic substances. Salts diffuse out of these poisoned fish into the distilled water in which they are placed, and (possibly as a result of increased permeability) they develop into monstrosities. An increase in permeability to water was shown by Loeb (I9I2 b).

If stimulation means increase in permeability, we should expect anesthetics to prevent it, since they prevent response to stimuli. It was observed that the increase in permeability of the eggs of certain fish to electrolytes could be partially inhibited by ether (McClendon, I9I4 b, I9I $5 \mathrm{~b}$ ).

Since Kite claims that the interior of the cell has the same permeability as the surface, it is probably worth while to emphasize that the effect of temperature change on the emf of the current of injury furnishes evidence that the seat of restricted permeability is the uninjured cell surface. Furthermore, Höber (I9IO a, I9I2 b, I9I3 b) has shown that the electric conductivity of the interior of the cell is greater than that of the whole cell, including the plasma membrane. 


\section{Stimulation}

Modern theories of electrical stimulation depend on membrane polarization. Nernst accidentally passed through his body a high frequency Tesla current of enormous voltage, such as is used in wireless telegraphy, and observed no stimulation. This led him to formulate his theory of electric stimulation, according to which the current must carry a certain number of coulombs of electricity per unit cross section, before it is reversed, in order to stimulate. In other words, stimulation is due to polarization of membranes, and the polarization must reach a certain minimal value. From this it is evident that the higher the frequency of an alternating current (the shorter the time the current flows continuously in one direction) the greater the amperage required in order to stimulate. Using sine wave alternating currents, this was found to be true within certain limits of frequency, but it appeared impossible to stimulate if frequency reached I00,000 per second. Nernst and Barrat (I904) found that the current required to stimulate $=.079 \times$ the square root of the frequency. In order to extend the limits of frequency complicated formulae have been developed to include accessory factors, such as backward diffusion of ions (A. V. Hill, r9Io). According to Bethe (I9I6) the facts fit better the hypothesis that only $\mathrm{H}$ ions are concerned in the stimulation. He had found that the passage of a current through a membrane causes the accumulation of $\mathrm{H}$ ions on one side and $\mathrm{OH}$ ions on the other and supposes that the accumulation of $\mathrm{H}$ ions on the outer surface of the plasma membrane causes stimulation. He says that the bare nerve fibers exposed at the cut end of a nerve, or which have grown out of the end on regeneration, can be stimulated by acid that is not hypertonic, and hence does not stimulate osmotically.

The relation of ions to irritability has been much studied, but the problem has not been entirely cleared up. An ion may have one effect at a certain concentration and a different effect at another. $\mathrm{K}$ ions are said to depress irritability, but according to Beccari (I9I5) they increase irritability, when applied within physiological limits to skeletal muscle. It is well known that $\mathrm{Na}$ increases and $\mathrm{Ca}$ decreases irritability. But irritability is lost in the pure solution of any salt. If muscle is placed in isotonic 
solutions of the chloride of an alkali metal the irritability disappears least rapidly in $\mathrm{NaCl}$ and most rapidly in $\mathrm{KCl}$ according to the series:

$$
\mathrm{Na}<\mathrm{Li}<\mathrm{Cs}<\mathrm{NH}_{4}<\mathrm{Rb}<\mathrm{K}
$$

If muscle remains in isotonic cane sugar solution the irritability disappears but if they are returned to a salt solution the irritability may be regained. According to Overton the cationic series is about the same as the one above. According to C. Schwarz the anionic series is: $\mathrm{CNS}<\mathrm{I}<\mathrm{NO}_{3}<\mathrm{Br}<\mathrm{Cl}<\mathrm{CH}_{3} \mathrm{COO}$ $<\mathrm{SO}_{4}$, Tartrate $<$ Citrate.

If it is carbonic or sarcolactic acid inside the muscle fiber that causes the polarization of the plasma membrane, this structure must be impermeable to all ions except $\mathrm{H}$ ions. The $\mathrm{H}$ ions of the sugar may be of the same concentration as the $\mathrm{H}$ ions inside the fiber and thus abolish the polarization. If this is the case, however, it is hard to see why the muscle is irritable in a mixture of sugar and neutral salt unless the salt in some unknown way changes the $\mathrm{H}$ ion dissociation of the sugar. The sugar solution acts as an insulator and hence makes stronger currents necessary for stimulation of the muscle directly. It might also interfere with the transfer of the negative variation from the nerve to the muscle by reducing the conductivity of the solution immediately over the plasma membrane. I know of no other suggestions that might explain the power of a cane sugar solution in reducing the irritability of muscle.

In this connection it may be of interest that the author found it necessary to make the medium more acid than $\mathrm{PH}=6.5$ in order to stop the pulsations of medusae or the heart of the Conch. The action of ions on these pulsations was studied with a view to determining the probability of the hypothesis of the antagonistic action of ions in isotonic solutions. $\mathrm{OH}^{\prime}, \mathrm{Na}^{\circ}$ and $\mathrm{K}$ stop the conch heart in systole, whereas $\mathrm{H}^{\circ}, \mathrm{Mg}^{*}$ and $\mathrm{Ca}{ }^{*}$ stop it in diastole. The first group tend to quicken and the second to retard the rate. As observed by Ringer, Mayer and others, however, $\mathrm{Ca}^{*}$ in the presence of other ions may tend to increase the systolic phase of pulsations. We cannot pick out 9 pairs of perfectly antagonistic ions, but the antagonism between $\mathrm{Na}^{\circ}$ and $\mathrm{Mg}^{*}$ is more perfect and between $\mathrm{OH}^{\prime}$ and $\mathrm{Ca}{ }^{*}$ less perfect. These antagonisms were more nearly perfect in the action of 
ions on the rate of pulsations of medusae, but the anomalous action of $\mathrm{Ca}$ " was again apparent. Perhaps the explanation of this lies in the assumption that the effect of the ions on pulsations is the summation of their effect on variou's tissue and cell structures, and that the sensitiveness of these structures is different for the same ion, the ions standing in no constant ratio in this regard. This is illustrated by the fact that $\mathrm{Mg}^{\cdot *}$ is a strong depressant of muscular contraction whereas Mayer (I9I5, I9I6) has shown it to be without effect on nerve conduction rate.

\section{The All-or-None Law}

If the action current (which we may assume is the excitation wave itself) rests on so mechanical a basis as has been set forth it may seem difficult to picture different gradations of excitation. Much evidence is being accumulated by physiologists to show that no gradations of excitation exist so far as the single cell is concerned, but that all excitations are maximal (Bayliss, I9I5 a). This is called the all-or-none law. A weak nerve impulse is explained as an impulse in which only a few fibers take part and a maximal impulse one in which all of the fibers take part; and the same principle holds for muscle. Tonic impulses are due to a succession of excitations involving a minute proportion of the fibers at any one time, and producing a fibrillation in the muscle that gives it tone. Such a tonic excitation continues to affect a veratrinized nerve muscle preparation for some time after a single stimulus is applied, thus retarding the relaxation of the muscle. The peculiar series of action currents after veratrin poisoning may be detected with the string galvanometer (Lodholz, 1913). Such a delicate instrument is not necessary in case of the electric organ, however, which shows the same behavior toward veratrin (Winterstein, Igro iii (2) I85).

The all-or-none law removes a series of objections which might be raised against a purely physicochemical hypothesis of excitation. It is the present purpose merely to call attention to this fact. For a discussion of the evidence in support of the law see Bayliss's Physiology.

\section{Absorption and Secretion}

The fact that the intestine may absorb a hypertonic solution introduced into its lumen has for a long time been the cause of 
astonishment. Hamburger (I908) found this valvelike action to be shown (in a very much smaller degree) by dead intestinal wall and by double membranes made by pasting together two membranes of different material. These rest1ts remind us of the theory of Flusin ( 1908 a) that osmose is controlled by three factors:

I. The attraction of the membrane for the solvent, determining its imbibition.

2. The presence of solutes affecting this imbibition.

3. The mutual attraction between solute and solvent.

This third factor is the principal one according to Traube, who calls it "haftdruck." Assuming 2 and 3 to remain constant, osmose is affected by imbibition of the membrane, and if imbibition is different on the two sides the osmose might be forced in one direction or the other. This seems to be the view of $\mathrm{H}$. N. Morse as a result of his direct determination of osmotic pressure. Osmose is then the resultant of three processes, between the first solution and the membrane, between the two sides of the membrane, and between the membrane and the second solution.

It seems evident that one effect of imbibition is to alter the size of the pores, and Bartell has shown that the enlargement of the pores can change positive into negative osmose. Electroendosmose seems to be at least one factor in the valvelike action of living membranes. Girard (I9IO) observed that the valvelike action of frog's skin separating isotonic salt from sugar solution, ceased when the skin current was inhibited by anesthetics.

Assuming that living membranes are easily permeable to $\mathrm{H}$ ions, the more acid of two solutions separated by the membrane should be electronegative, and the osmose should be toward it, since electroendosmose is usually toward the negative pole. This view is in harmony with the fact that osmose is from the alkaline blood to the acid stomach and kidney cavities, but from the alkaline intestine to the slightly less alkaline blood. It is probable that protoplasm is less alkaline than blood, hence the turgidity of cells.

Bernstein in his "Elektrobiologie" accords first place to electroendosmose in explaining osmose through living membranes. The electric polarization of such membranes is reduced or disappears 
on death-hence, electroendosmose must be reduced or cease also. The cause of this cessation of electroendosmose at death is probably not a sudden abolition of the difference in concentration of ions causing the emf, but an alteration of the membrane. It was shown long ago by Nägeli, and repeatedly confirmed by others, that increase in permeability of the plasma membrane is so intimately associated with death of the cell that the two phenomena are probably the same thing. This seems true notwithstanding the fact that the plasma membrane may not lose all of its semipermeable properties on death. Some of the evidence that disappearance of the bioelectric phenomena alter osmose are as follows:

If the turgor of living cells is due to electroendosmose, dead cells should dry in air sooner than live ones. This fact is the teleological principle involved in the instinct of certain wasps who store spiders with the eggs so that the larvae may have food. If the spiders are killed they dry up before the eggs hatch, hence the necessity of keeping them alive. The wasp stings the spider in its central nervous system so that it remains alive in a paralytic condition. Bernstein ("Elektrobiologie") tied living and dead skin over the mouths of two tubes filled with water. The evaporation took place more rapidly through the dead skin. He suspended two similar pieces of the frog's skin from the two arms of a balance. One of the pieces of skin was killed, and it then lost weight faster than the other. A similar experiment with plant leaves gave the same result. The same was true of frog's muscle if the experiment were terminated within a half hour, at the end of which time the live muscle had been killed by drying.

Recent experiments by F. H. Scott on blood volume and by A. D. Hirschfelder on edema confirm the older ideas that the transfer of fluid in the mammalian body is largely accounted for by filtration and the osmotic pressure of colloids. The osmotic pressure of colloids is considered in Chapter VI. 


\section{CHAPTER XI}

\section{ANESTHESIA AND NARCOSIS}

We are familiar with the power of anesthetics in restraining the activity of the central nervous system, especially the reflex and voluntary activities which they affect in lower concentrations than those affecting other organs. Since the higher animals die as soon as the activity of the respiratory center ceases, and under artificial respiration as soon as the heart beat ceases, it is possible in the intact animal to study the effects of anesthetics only on certain nerve synapses, i. e., structures whose threshold of anesthesia is lower than that of the heart mechanism. Anesthetics in higher concentrations suspend the activity of cells and of animals lacking a nervous system. Clatıde Bernard showed that ether may prevent the movement of the leaves of Mimosa, a sensitive plant.

Overton (I9OI) observed that vertebrates, insects, and entomostraca (small crustacea) require practically the same concentration of anesthetic (or the same vapor pressure of volatile anesthetic) in order to suspend reflex excitability, whereas certain groups of worms require double this concentration. Protozoa and plants require, in order to suspend their activities, six times the concentration of anesthetic required to anesthetize vertebrates. From Warburg's data, the concentration of anesthetic necessary to reduce the oxidation of goose erythrocytes 30-70 per cent is about six times the concentration necessary to anesthetize vertebrates, although some variation in regard to individual anesthetics is apparent. Slight discrepancies might be due to the fact that the anesthesia data were taken by Overton on samples of anesthetic of a lower degree of purity than those used by Warburg to reduce oxidation. It follows from this that protozoan and plant cells react to anesthetics, not like nerve synapses but like mammalian cells. According to Winterstein (I9I4), however, narcosis of frog's spinal cord may either decrease or increase oxidation. $\mathrm{O}_{2}$ is necessary for complete recovery. 
It appears, therefore, that cells in general require about the same concentration of anesthetic to suspend their activities. Since this process seems to be the reverse of stimulation and stimulation is associated with increase in permeability, we might expect anesthetics to prevent increase in permeability. Traube (I908 a) observed that isoamyl alcohol, which belongs to a class of substances which in high concentration cause hemolysis, at a certain low concentration tends to prevent hemolysis of the erythrocytes by other agents.

Lillie ( Ig09 b) observed that pure solutions of certain salts cause an outward diffusion of the pigment from Arbacia eggs, and interprets this as increase in permeability. He also found that anesthetics inhibit this action of the salt (19I4, b), so that the salt solution + anesthetic may not cause loss of pigment. This is in harmony with the recent observation of S. Loewe (I9I3) that anesthetics decrease the electric conductivity of lipoid membranes soaked in salt solution. These results are probably due to increase in size of colloidal lipoid particles. Clowes (I9r6) supposes the plasma membrane to be an emulsion of lipoid in water that is changed by anesthetics to an emulsion of water in lipoid.

Osterhout (I9I3 a) observed that small quantities of anesthetics added to the sea water decrease the electric conductivity of seaweed. He had already observed that $\mathrm{Na}$ increases the conductivity and $\mathrm{Ca}$ decreases it. Since $\mathrm{Ca}$ and anesthetic both inhibit the conductivity increasing action of $\mathrm{Na}$, it seems probable that the anesthetic in the sea water inhibits the action of the $\mathrm{Na}$ already in the sea water. It follows from this that anesthetics inhibit the increase in permeability to ions.

Hemolysis consists in the loss of the hemoglobin from the red blood corpuscles. Not only does the permeability of hemoglobin increase but Stewart (I899) has shown that the permeability to ions increases. Arrhenius and Bubonovic observed that anesthetics inhibit hemolysis by hypotonic solutions. Joel (I9I5) showed that anesthetics inhibit the permeability increasing action of pure cane sugar solution on erythrocytes.

The eggs of certain fish are admirable for permeability studies, since they develop in distilled water, in which they neither swell nor lose salts. It had been observed that the permeability of 
Fundulus eggs may be increased by certain solutions of salts, alkaloids or other stubstances in concentrations that do not kill them (McClendon, IgI2 a, e, I9I3 a). The eggs of the pike behave similarly. These eggs are impermeable to water or to salts, but when placed in o.I mol $\mathrm{NaNO}_{3}$ they lose chlorides, which pass out into the nitrate solution and may be accurately estimated with Richards's nephelometer. Three per cent ethyl alcohol causes retardation in the development of these eggs and is therefore considered an anesthetic. If the eggs are placed in o.I mol $\mathrm{NaNO}_{3}$ containing 3 per cent alcohol only half as much chlorides diffuse out of them as when placed in O.I mol pure $\mathrm{NaNO}_{3}$. The anesthetic inhibits the permeability increasing action of the pure salt solution (McClendon, I9I4 b, I9I5 b).

The skin current of the frog disappears when the skin is anesthetized, indicating that the hypothetical increase in permeability of the outer surfaces of the gland cells during sectetion may be inhibited by anesthetics. In this case we do not know whether nervous stimulants are exclusively the cause of the increase in permeability.

It seems clear, therefore, that anesthetics inhibit the increase in permeability of the plasma membranes of the irritable elements. In order for them to do this it is necessary for them to penetrate only the surface film of the cell. But anesthetics belong to the group of substances which penetrate cells easily, and the question arises as to their effect on the interior. Some experiments on catalysis are suggestive. If blood charcoal is mixed with a solution of oxalic acid containing atmospheric oxygen the acid is burned to $\mathrm{CO}_{2}$ and $\mathrm{H}_{2} \mathrm{O}$. Blood charcoal contains mineral matter, including iron, which is not entirely removed by extraction with $\mathrm{HCl}$ and we cannot say that it is the carbon that acts as the catalyzer. Warburg found that the catalyzing power of charcoal varies with its power of adsorbing oxalic acid, hence the combustion must take place near the surface of the charcoal. He observed further that anesthetics (of the trethane series) inhibit the oxidation, and explained this as due to the displacement of the oxalic acid from the surface of the charcoal by the anesthetic (which is ad'sorbed to a greater extent than the acid). The author confirmed these results, using charcoal extracted with concentrated $\mathrm{HCl}$ followed by $\mathrm{H}_{2} \mathrm{O}$ for 
many hours. This charcoal was heated to redness and cooled in a $\mathrm{CO}_{2}$-free atmosphere. The charcoal adsorbed all of the $\mathrm{CO}_{2}$ produced for a time, but after it had become saturated with this gas, the volume of $\mathrm{O}_{2}$ used up was half the volume of the $\mathrm{CO}_{2}$ given out. No food' substance was found that 'could be burned in this way. Rona and Tosh (rgI4) found, however, that urethane displaces adsorbed dextrose from animal charcoal.

Meyerhof (I9I4 b) observed that the catalysis of hydrogen peroxide by colloidal platinum may be inhibited by anesthetics. Since the anesthetic did not reduce the number of particles of platinum seen in the ultramicroscope he concluded that the anesthetic displaced the $\mathrm{H}_{2} \mathrm{O}_{2}$ from the surface of the platinum.

Meyerhof (I9I5) showed that solution of invertase from yeast may be inhibited by anesthetics. The following table shows the concentration of anesthetic that reduced the inversion of 3 per cent cane sugar solution 30 per cent:

$\begin{array}{lc}\text { Methyl alcohol " } & 3 \text { mol } \\ \text { Ethyl " } & 1.3 \text { " } \\ \text { Propyl " } & .5 \text { " } \\ \text { Isobutyl " } & .27 \text { " } \\ \text { Isoamyl (fermentation) } & .21 \text { " } \\ \text { Methyl urethane } & .95 \text { " } \\ \text { Ethyl " } & .97 " \\ \text { Propyl " } & .48 \text { " }\end{array}$

In this case the Tyndall phenomenon was increased, but this may have been due to an increase in the dispersion of light by particles, due to their inclosure in films of anesthetic, rather than an increase in the size of the particles. In harmony with the view that the reduction in activity of the enzyme is due to the displacement, by the anesthetic, of the sugar from its surface, is the fact that if the concentration of the anesthetic is varied its effectiveness follows the typical adsorption curve (Fig. 24). The concentration of anesthetic necessary to anesthetize tadpoles reduced the inversion about 20 per cent, but this reduction was increased by dilution of the sugar solution.

If the anesthetics are added in greater concentrations to enzyme-containing extracts of cells, precipitation may occur. The enzymes are carried down in the precipitates, but it is not 
clear whether the enzyme is precipitated directly by the anesthetic, or protein impurities precipitated and the enzyme adsorbed by the protein. Batelli and Stern (I9I3) showed that anesthetics precipitate nucleoprotein.

Warburg and Wiesel (1912) observed that anesthetics inhibit the fermentation by yeast juice, attributing this inhibition to the precipitation of the zymase, since the inhibiting and precipitating powers of anesthetics are parallel. However, live yeast requires for inhibition less concentration of anesthetic than is required by yeast juice (or for precipitation of protein).

Vernon (I9r2 a) observed that anesthetics are harmless to the indophenol oxidase of chopped kidney until a certain concentration is reached, above which they inhibit its action. The data of Batelli and Stern are given in the following table, as compared with the anesthetization of tadpoles by Overton and the reduction of the respiration of sea urchin eggs, fish sperm, goose erythrocytes, lymphocytes, liver cells, spinal cord, yeast and bacteria $30-70$ per cent, by Warburg. The figures are the gram molecular concentrations:

Methyl alcohol

Ethyl alcohol

Propyl alcohol

Isobutyl alcohol

Isoamyl alcohol

Acetone

Methylethylketone

Methylpropylketone

Allyl alcohol

Ethyl urethane

$\begin{array}{cc}\begin{array}{c}\text { Inhibition of } \\ \text { oxidation }\end{array} & \begin{array}{c}\text { Precipitation } \\ \text { of nucleo- } \\ \text { at I.5 }\end{array} \\ \text { proteid. } \\ \text { Ir. } 44 & \text { I2.48 } \\ 6.12 & 6.97 \\ 2.04 & 2.72 \\ \text { I.06 } & \text { no ppit. } \\ 0.41 & \text { no ppt. } \\ 5.70 & 4.34 \\ 2.22 & 2.4 \mathrm{I} \\ 0.77 & \text { no ppt. } \\ 2.70 & 4.05 \\ 1.90 & 2.58\end{array}$

Inhibition of Anesthesia respiration of tadpoles, of cells, Overton. Warburg.

0.62
0.31
0.11
0.045
0.023
0.26
0.09
0.019

0.037
5

I.6

0.8

0.15

0.045

0.33

This table shows that about six times the concentration of anesthetic effective on tadpoles is required to inhibit the respiration of goose erythrocytes. Gros (I9IO) found the same thing true for the anesthesia of peripheral nerves. It seems, therefore, that the nerve cells of the tadpoles are not anesthetized, but the synapses are in the same condition as during sleep. For anesthetization of nerve cells and reduction of the respiration of goose erythrocytes the same concentration is required, but for inhibition of the oxidases and precipitation of protein in Batelli 
and Stern's experiments more than double this concentration is needed. In fact, the concentration used by Batelli and Stern is much above the lethal dose for cells. Warburg (I9II a) explains the fact that anesthetics inhibit enzymes, when contained in living cells, in lower concentrations than the same anesthetics inhibit the same enzymes in cell extracts, by the assumption that the enzymes within the cells are adsorbed to phase boundaries, the anesthetics becoming more concentrated at the phase boundaries. If this explanation is correct we must make the further assumption that the enzymes in the cell extracts referred to are in true solution, whereas the invertase used by Meyerhof formed a colloidal solution because the invertase was inhibited by as low a concentration of anesthetic as is required to inhibit the respiration of the erythrocytes.

Although Batelli and Stern claim that anesthetics precipitate proteins Traube and Köhler (I9I5) and Traube (I9I5) assert that anesthetics help in the solution of proteins. In the following table they have arranged anesthetics according to their power of hastening the swelling and solation of gelatine jelly (and retarding gelation of sols). It is found that they are with few exceptions in the order of anesthetic power, as shown by the figures in the last column, which represent the molecular concentration for anesthesia.

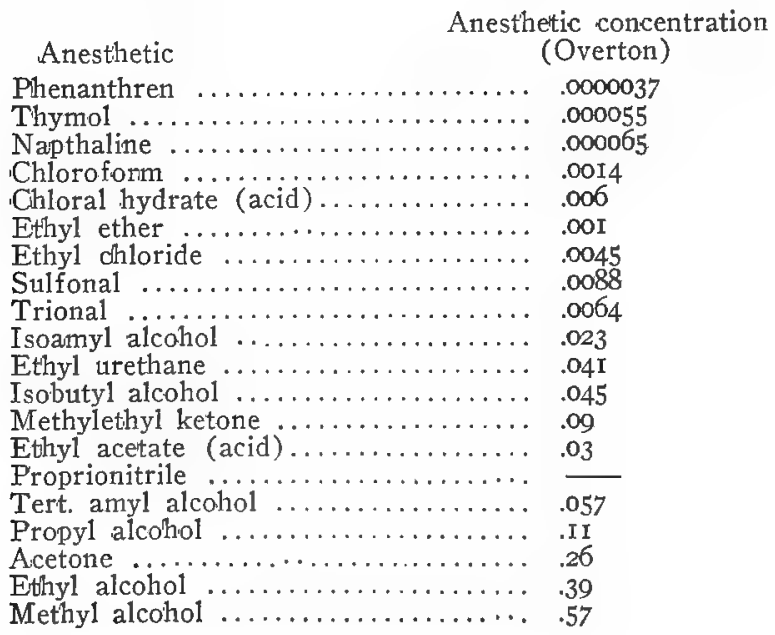


Perhaps the anesthetics protect the gelatine from the precipitating action of accidental impurities. On the contrary, if impurities hold a protein in solution the anesthetic might then help precipitate the protein. This may explain the opposite effect of anesthetics on nucleoprotein solutions.

This action of anesthetics in displacing other substances from the surface film probably explains the observation of Abl (1907) that they reduce the electrolytic solution tension of cadmium, and the observation of Grumbach (I9II) that they reduce the contact potential between dielectric and electrolyte.

The action of anesthetics on living cells may be explained by the same assumption, i. e., that they cover the plasma membrane with a film which protects it from change, entering the cell and displacing enzymes or reacting substances from the phase boundaries where these reactions occur more rapidly than elsewhere. The synapses that have to do with consciousness are more sensitive to anesthetics than are either plasma membranes or these enzyme reactions. Hence, it requires only one-sixth the concentration to anesthetize that it requires to inhibit other processes.

This hypothesis also explains the relation between anesthetic power and surface tension as emphasized by Traube, who states that the more an anesthetic lowers the surface tension of water the greater is its anesthetic power. This is nearly correct, due to the fact that anesthetics that lower the water-air surface tension are adsorbed to many water-solid or water-liquid surfaces.

According to Overton (I9OI) and H. Meyer (I9OI), anesthetic power varies with the partition coefficient of the anesthetic between lipoid and water. This partition coefficient has never been determined, but these authors have been content with the partition coefficient between olive oil and water or between some solution of lipoid and water. In many cases even this was not determined, but the ratio between the solubility in lipoid solution and in water substituted. Owing to the concentration at the phase boundary, these two magnitudes are probably never the same. But it seems generally true that stibstances which very greatly lower the surface tension between water and air, have a high partition coefficient between oil and water and are therefore soluble in oil-they are also solutble in many other organic solvents. The "solubility" of an anesthetic in lipoid 
"solutions" seems to be due to the fact that it is adsorbed by the colloidal particles of lipoid so that the solution can hold more anesthetic than the solvent (benzol, etc.) can. Thus we see that adsorption is at the basis of the whole matter.

In some cases the partition coefficient of the anesthetic between cells or tissues and water has been determined. Warburg, by this means, concluded that the erythrocytes consist of a larger volume of watery phase than of lipoid phase.

It has been shown by a number of investigators that alcohol, ether, chloroform, chloral hydrate and acetone become more concentrated in nerve than in other tissue, this being used to explain the ease with which the central nervous system may be anesthetized. The nervous system, however, stores the anesthetic partly in lipoids which are more abundant in the white matter, forming the myelin sheaths of the nerves, and the nerves are no more easily anesthetized than non-nervous cells (Gros). Nerve impulses are still carried in the anesthetized body, only certain nervous processes being stopped, as in sleep. This stoppage may be explained by the assumption that certain synapses are affected by the anesthetic.

It is characteristic of pure anesthesia that it is a reversible process, brought about only by a certain concentration of anesthetic. Many anesthetics, even in the optimum concentration, have not only an anesthetic but also a toxic action, so that prolonged anesthesia is harmful. This is characteristic of all anesthetics with acid or basic properties, the alkaloids, for instance, whose chemical affinity may explain their selective action on certain cell structures. Other anesthetics are decomposed in the body into harmful substances. Esters are saponified with the liberation of acids.

But even the purest types of anesthetics are toxic if the anesthetizing dose is exceeded (provided they are sufficiently soluble to exceed this dose). This toxic action is manifested by increase in permeability, which is reversible only if very slight and momentary. The action of anesthetics in these high concentrations will be considered in the chapter on antiseptics and in other chapters.

When ether is given to an animal, a stage of excitement sometimes precedes that of narcosis. This fact is open to various 
interpretations in an animal with a cerebrum, but Hamburger (I9I2) observed that ameboid motion is increased by minute doses of anesthetics. Johannsen (xgo6) observed that lilacs sprout earlier after treatment with ether vapor. Further examples of the stimulating action of anesthetics are given by Carlson (1906), Vernon (1912), Tashiro and Adams (19I4), Lee (I903), and Lee and Salant (I902). The possibility is not excluded, therefore, of three concentrations of anesthetics producing three kinds of responses: first, stimulation; second, narcosis, and third, violent stimulation causing death if continued.

The following table (modified from Lillie, I9r6 c) summarizes the effect of anesthetics (alcohols) on various processes, giving the effective mol concentration.

Effect

Narcosis of tadpoles (Overton) ...... 0.57

Phototropism of Daphnia (Loeb) ...... 0.6

Inhibition of sea urchin eggs (Fühner) 0.72

Retardation of fish eggs (McClendon) -

Half rate of turtle ventricle (Vernon) I.I

Narcosis of Daphnia (Loeb)......... I.2

Inhibition of sea urchin eggs (Lillie).. -

Narcosis of worm larvae (Lillie).... 2.2

Depression of invertase (Meyerhof) ... 3.0

Half oxidation in erythrocytes (Warburg)

Ppt nucleoproteid (Batelli............. 5.0

Inhibition of ether-yeast (Warburg)

Kills muscle oxidon (Batelli and Stern) 7.54

Kills indophenol oxidase (Vernon).... I2.0

Reduce surface tension to $0.5 \ldots \ldots \ldots$ I 4.0

$\begin{array}{clll}\text { Ethyl } & \text { Propyl } & \text { Butyl } & \text { i-Amyl } \\ 0.29 & 0.11 & 0.038 & 0.023 \\ 0.2 & 0.075 & 0.04 & - \\ 0.41 & 0.136 & 0.045 & - \\ 0.52 & - & - & 0.015 \\ 0.53 & 0.23 & 0.05 & 0.02 \\ 0.6 & 0.2 & 0.12 & 0.04 \\ 0.87 & 0.27 & 0.086 & 0.037 \\ \text { I.I } & 0.34 & 0.09 & 0.03 \\ \text { I.3 } & 0.5 & 0.27 & 0.21 \\ & & & \\ \text { I.6 } & 0.8 & 0.15 & 0.045 \\ 2.38 & \text { I.I2 } & 0.45 & 0.21 \\ 3.5 & 1.3 & 0.54 & 0.23 \\ 3.57 & \text { I.I6 } & 0.44 & 0.19 \\ 6.4 & 3.1 & 0.61 & - \\ 5.0 & 1.6 & 0.46 & 0.14\end{array}$




\section{CHAPTER XII}

\section{CYTOLYSIS AND DISINFECTION}

The first action of many toxic substances on cells is the destruction of the plasma membrane. The cell is then no longer influenced by the osmotic pressure of the medium but may swell or shrink as any other colloid mass, depending on certain conditions. Animal cells after destruction of the plasma membrane usually swell and disintegrate, a process known as cytolysis, meaning the dissolution of the cell. It seems probable that death of all cells is accompanied by cytolysis, but that other processes sometimes mask the cytolytic changes. Thus, in the histological fixation of cells the protoplasm is transformed into a stable mass immediately after the destruction of the plasma membrane, so that no swelling or shrinking follows the increase in permeability. Since cytolysis means death of the cell, all cytolytics may be used as antiseptics, destroying at least the active forms of microorganisms.

The indifferent anesthetics, when their concentration exceeds that necessary for the inhibition of cell activities, increase the permeability of the plasma membrane. This increase, if slight and momentary, may be reversible, at least in some cases. If the semipermeability of the cell is entirely removed, cytolysis and death occur. For this reason anesthetics have an antiseptic action. Some active cells are more resistant to anesthetics than others, the spores of bacteria usually not being killed, but merely prevented from germinating so long as the anesthetic is present.

The fact that anesthetics increase the permeability of cells is illustrated by the fact that they accelerate autolysis. If tissue is cut off from the blood supply, either by ligature of the vessels or by removal from the body, and kept free from germs, it digests itself, a process known as autolysis. Certain substances accelerate autolysis. Chiari (ro09) observed that ether accelerates autolysis. Hans Meyer (I909) explained its action as an increase in permeability of the tissue, allowing the contact 
of protease with protein and a general diffusion and mixing of the substances in the tissue.

The cytolytic and antiseptic power of anesthetics varies with their power of penetrating into the plasma membrane substance, and hence varies approximately with their power of lowering the surface tension of water. Solutions of the same surface tension are called isocapillary solutions. It was found that isocapillary solutions of ether, alcohols, and acetone just caused death of Fundulus eggs, with increase in permeability (McClendon, I9I2 a). Czapek (I9IO) observed that indifferent anesthetics just caused death and increased permeability of plant cells when they lowered the surface tension of water to .68 . Scheurlen ( 1896 ) observed that $\mathrm{NaCl}$ increases the toxicity of phenol. This may not be an additive effect, as $\mathrm{NaCl}$ may reduce the surface tension of phenol solutions in the same way that it reduces the surface tension of bile (Allen, G. D., I9I5 a).

It seems, therefore, that the toxicity of all indifferent anesthetics depends on the concentration and that the concentration of the anesthetic in the plasma membrane is the only variable. With other substances, however, this is not the case. If the anesthetic is of such nature as to be chemically active in the ordinary sense (not through residual valences) it is more toxic than should be from the surface tension of its solution. Substances with basic, acid, reducing or oxidizing properties are especially toxic, ammonia, phenol, formaldehyde and $\mathrm{H}_{2} \mathrm{O}_{2}$ being examples. But the acidity of phenol is not high enough to account for its toxicity, and formaldehyde, besides having a reducing action, forms additive compounds with protoplasm in the same way that it combines with ammonia to form hexamethylentetramine.

The toxicity of cations varies inversely with the electrolytic solution tension, therefore the following series arranged in decreasing order of solution tensions is for the most part in increasing order of toxicity: $\mathrm{Cs}, \mathrm{Li}, \mathrm{Rb}, \mathrm{K}, \mathrm{Na}, \mathrm{Ba}, \mathrm{Sr}, \mathrm{Ca}, \mathrm{Mg}$, $\mathrm{Al}, \mathrm{Mn}, \mathrm{Zn}, \mathrm{Cd}, \mathrm{Sn}, \mathrm{Fe}, \mathrm{Tl}, \mathrm{Co}, \mathrm{Ni}, \mathrm{Pb},(\mathrm{H}), \mathrm{Als}, \mathrm{Cu}, \mathrm{Bi}, \mathrm{Sb}$, $\mathrm{Hg}, \mathrm{Ag}, \mathrm{Pl}, \mathrm{Pt}$, Au.

Mathews (I904 a) found the order of toxicity of salts of these ions on Fundulus eggs to be $\mathrm{Mn}, \mathrm{Fe}, \mathrm{Co}, \mathrm{Ni}, \mathrm{Zn}, \mathrm{Pb}, \mathrm{Cd}$, $\mathrm{Cu}, \mathrm{Hg}, \mathrm{Ag}, \mathrm{Au}$. It may be noticed that $\mathrm{Zn}$ and $\mathrm{Cd}$ are out of 
the place they occupy in the series of electrolytic solution tensions. The $\mathrm{ZnCl}_{2}$ used was more toxic than it should be, owing probably to hydrolysis, by which the solution became acid. The great toxicity of the $\mathrm{CdCl}_{2}$ is probably not due entirely to the cadmium ion but partly to the unusually large proportion of undissociated molecules which are easily adsorbed.

In estimating the toxicity of ions, the degree of ionization must be calculated. The addition of large quantities of some electrolytes to solutions of some heavy metals inhibits the ionization of the latter by the formation of double salts. If $\mathrm{NaCl}$ is added to $\mathrm{HgCl}_{2}$ solution, $\mathrm{Na}_{2} \mathrm{HgCl}_{4}$ is formed, which is very poorly dissociated by steps, forming: $\mathrm{Na}$ and $\mathrm{NaHgCl}_{4}{ }^{\prime}, \mathrm{Na}^{*}$ and $\mathrm{HgCl}_{4}{ }^{\prime \prime}, \mathrm{Hg}^{*}$ and ${ }_{4} \mathrm{Cl}^{\prime}$. The number of $\mathrm{Hg}$ ions formed is very small and the addition of quantities of $\mathrm{NaCl}$ reduces it to a vanishing magnitude.

The addition of $\mathrm{NaCl}$ increases the solubility of $\mathrm{HgCl}_{2}$ but not the concentration of $\mathrm{Hg}^{\circ}$. If solid $\mathrm{HgCl}_{2}$ is shaken with water, the salt dissolves until there is an equilibrium between the solid phase and the undissociated molecules in solution. Some of these molecules dissociate, thus disturbing the equilibrium, and more of the solid dissolves to restore it, until a second equilibrium is established between the ions and the molecules. This equilibrium is expressed by the equation $\frac{\left[\mathrm{Hg}^{*}\right] \times\left[\mathrm{Cl}^{\prime}\right]}{\left[\mathrm{HgCl}_{2}\right]}=$ a constant, but since the concentration of the undissociated molecules is constant so long as there is any undissolved salt, the equation becomes $\left[\mathrm{Hg}^{\bullet}\right] \times\left[\mathrm{Cl}^{\prime}\right]=$ a constant. If we add $\mathrm{Hg}\left(\mathrm{NO}_{3}\right)_{2}$, thus increasing the concentration of $\mathrm{H}_{\mathrm{g}}$ ions, this solubility product will be exceeded and some $\mathrm{Hg}^{\circ}$ and $\mathrm{Cl}^{\prime}$ will combine, the additional $\mathrm{HgCl}_{2}$ thus formed crystallizing out. If, on the contrary, we add $\mathrm{NaCl}$, the $\mathrm{Cl}^{\prime}$ will in the same way lower the solubility of $\mathrm{Hg}$ ions, but the $\mathrm{Na}$ combines with the $\mathrm{Hg}$ and $\mathrm{Cl}$ ions forming $\mathrm{Na}_{2} \mathrm{HgCl}_{4}$, thus removing them, more $\mathrm{HgCl}_{2}$ dissociates, and more bichloride dissolves. This process is continued until the solution is saturated with $\mathrm{Na}_{2} \mathrm{HgCl}_{4}$. The concentration of $\mathrm{HgCl}_{2}$ at equilibrium is the same as before, but the concentration of $\mathrm{Hg}$ ions decreases when the concentration of $\mathrm{Cl}$ ions increases, since their product is constant. The solution 
loses most of the $\mathrm{Hg}$ ions if the concentration of the $\mathrm{NaCl}$ is high, but gains the $\mathrm{Na}_{2} \mathrm{HgCl}_{4}$, which more than compensates this loss, so that the total $\mathrm{Hg}$ in solution is greater than before.

Although the solubility of $\mathrm{HgCl}_{2}$ is increased by the addition of $\mathrm{NaCl}$, and the mercury may be used in greater concentration, this does not mean that the toxicity is increased. The question as to whether the ions or molecules are the more toxic must first be answered. This seems to vary with the duration of the exposure to the poison, as shown by the following experiments:

Paul and Krönig (1896) determined the disinfecting power of differently dissociated salts of mercury on Anthrax spores. Those salts which are poorly dissociated are less toxic than those which are more completely dissociated. In other words, the toxicity depends chiefly on the mercuric ions. The following table shows the number of colonies that developed after exposure to the toxic solutions:

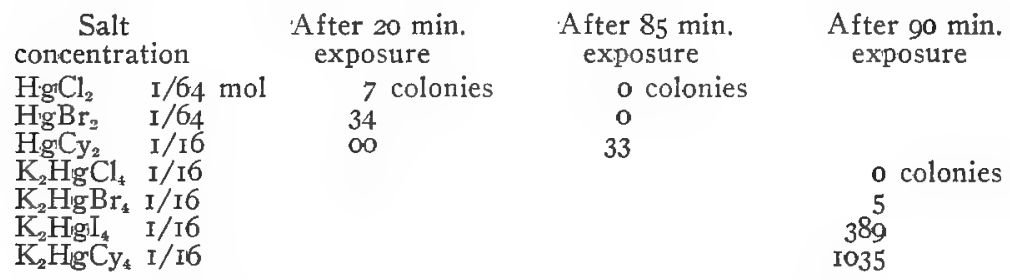

The same rule had previously been found by Kahlenberg and True (r89I) to apply to the toxicity of copper salts, and seems to be approximately true of all heavy metals. It is only approximately true because the molecules are toxic, although not so toxic as the ions, during brief periods of exposure, and the rule does not hold for prolonged exposure.

If salts of heavy metals are allowed to act on organisms for a long time, their toxicity is approximately proportional to the total quantity of the heavy metal they contain. Stevens (I898) and Clark ( I899) grew Penicillium and Aspergillus in such solutions, finding that the inhibition of growth is approximately proportional to the quantity of the heavy metal, without regard to the combination in which it exists. It is probable that the heavy metal ions combine with the protoplasm, thus leading to further dissociation of the salt until all is dissociated and combined. 
This seems to be the action of organic preparations of heavy metals such as "argyrol."

The toxicity of each cation may be decreased more or less by certain other cations. For this reason, the toxicity of a salt is reduced when it is added to solutions containing other salts (as is always the case where it is desired to disinfect). Only the most toxic salts, therefore, are reliable for disinfection. Copper salts may be used in some cases but salts of mercury are better. The salts of the precious metals are the most reliable.

The salts of the lighter metals may be toxic, especially if their solutions are pure and kept pure by replacing the contaminated solution with fresh ones. Some discrepancies seem to exist between the solution-tension series and the toxicity series, but these would probably be lessened if all of the salts were absolutely pure and if some indifferent substance could be found to equalize the osmotic pressure. Höber (I908 a) studied the relative efficiency of slightly hypotonic solutions in causing cytolysis of erythrocytes (hemolysis). The ionic series with increasing hemolytic power is as follows:

$$
\begin{aligned}
& \mathrm{SO}_{4}<\mathrm{Cl}<\mathrm{Br}<\mathrm{NO}_{3}<\mathrm{CNS}<\mathrm{I} \text { and } \\
& \mathrm{Li}, \mathrm{Na}<\mathrm{C} 5<\mathrm{Rb}<\mathrm{K}
\end{aligned}
$$

Lillie (I909 a) observed the same anionic series for the cytolysis of sea urchin eggs, but $\mathrm{Na}^{*}$ was more toxic than $\mathrm{K}$. The same anionic series was found to hold for the cytolysis (dissolution) of cilia (Lillie, Ig09 c; Höber, Ig09 b), but the cationic series is different for different species of animals. In general, it may be said that the iodides of the alkali metals, when pure, exert a cytolytic action.

It has been shown by Kahlenberg and True (1896) and others that the toxicity of strong acids depends on the concentration of the hydrogen ions. Mineral acids cause death of seedlings and cytolysis of many plant cells when the concentration exceeds I/6400 normal. For disinfection . In or stronger solutions may be used.

The toxicity of the weak acids depends on several factors, so that no general rule may be made in regard to them. Their power of reducing the surface tension of water is the index of one factor. Acetic acid and sulphurous acid are used in disinfection. 


\section{OF VITAL PHENOMENA}

The toxicity of the strong bases depends on the hydroxyl ion concentration. These are seldom used for disinfection because they are neutralized by the $\mathrm{CO}_{2}$ of the air. Quick lime is used more for its drying power and the toxicity of the $\mathrm{Ca}$ ions, than for the toxicity of the $\mathrm{OH}$ ions.

The weak bases are similar to the weak acids in that their power of reducing the surface tension of water must be considered. Some of them, such as quinine and strychnine, are especially destructive to certain protozoan parasites.

Solutions of soap are alkaline, due to hydrolysis. Soap lowers the surface tension of water enormously and has a marked cytolytic action. It destroys the free forms of many bacteria.

We may summarize some of the disinfectants as follows:

Anesthetics. Alcohol, 50-70\%. Phenol 5\%, 24 hrs.; Cresol $5 \%, 2$ hrs.

Oxidizers. $\quad \mathrm{H}_{2} \mathrm{O}_{2} \mathrm{I} \%$, 30 min. $\mathrm{Cl}_{2} ; \mathrm{Br}_{2} ; \mathrm{I}_{2}$.

Reducers. Formaldehyde, $\mathrm{SO}_{2}$.

Strong acids. O.I normal I5 minutes.

Weak acids. Acetic, concentrated.

Strong bases. $\mathrm{Ca}(\mathrm{OH})_{2}$ (dehydrating).

Weak bases. Quinine.

Soaps.

Heavy metals. $\mathrm{HgCl}_{2}$; Cacodylic acid $\mathrm{AgNO}_{3}$ Argyrol; $\mathrm{CuSO}_{4}$; Salvarsan. 


\section{CHAPTER XIII}

\section{AMEBOID MOTION AND TROPISMS, CELL DIVISION, FERTILIZATION AND PARTHENOGENESIS}

It was shown by Quincke that movements of drops of fluid, resembling ameboid motion, could be produced by localized changes in surface tension. A local reduction in surface tension produces a protrusion of the affected surface-a localized increase produces a flattening. By a combination of such changes in surface tension, combined with adhesions to the substratum and to food particles, Rhumbler (1905, 1910) has imitated all forms of ameboid motion.

In imitating food taking, Rhumbler found that his artificial amebae would take only such food as adhered to them. A chloroform drop would engulf a piece of shellac but not take a piece of glass. If a piece of glass is coated with shellac, the chloroform drop will engulf it, but after the shellac is dissolved off it will reject the glass. In this way the process of defecation by the ameba is imitated. Such a process also imitates the shell building of some protozoa. These shells are formed of defecated particles which cover the surface of the protozoon. If chloroform is shaken with powdered glass and dropped into water, the drops are found to be surrounded by shells composed of particles of glass.

The ameba apparently does not adhere to all of the food that it takes, shown by the fact that it often takes in a quantity of water with the food particle. The food is separated from the protoplasm on all sides by water, and thus, after ingestion, comes to lie in a food vacuole. Bernstein (1900) was able to make an artificial ameba take food to which it did not adhere. A drop of mercury was immersed in dilute nitric acid and a crystal of potassium bichromate held near it. As soon as some dissolved bichromate reached the mercury the surface tension was reduced and the mercury moved toward the crystal. The momentum of 
the mercury was often so great that it engulfed the crystal. This experiment is crude, the movement being very rapid and the crystal immediately thrown out after being ingested.

In order to explain the food taking of the ameba, a still further localization of surface tension changes must be assumed. If we assume that a substance diffuses from the food, which in very low concentration causes a reduction in surface tension of the ameba, but in higher concentration causes an increase in the surface tension, we can explain this form of food taking. When the ameba comes within the sphere of influence of the food its surface tension is reduced on that side, and it advances or sends a broad pseudopod toward the food. The center of the advancing edge of the ameba comes so close to the food that its surface tension is increased and its forward motion is stopped. On each side of this central area, the protoplasm continues to advance and finally forms a ring around the food. The upper and lower edges of this ring constrict and thus enclose the food together with some water in a hollow sphere of protoplasm.

Quincke ( 1888 a) supposed the surface of protoplasm to be of an oily nature, and hence soap should lower the surface tension. It was. found, however, that soap, no matter what the concentration, always increased the surface tension of the ameba, when it had any effect at all (McClendon, I9I I c). Since no phase boundary is known whose tension is increased by the presence of soap in either phase, some indirect action of the soap must be looked for.

Lippman showed that electrical polarization of a phase boundary reduces the surface tension, and that reduction of this polarization increases the surface tension. We have seen that there is abundant evidence to show that the plasma membrane of living cells in the resting condition is electrically polarized, and that increase in permeability reduces this polarization. Increase in permeability, therefore, increases the surface tension of cells. This is demonstrated by the rounding up of an ameba when it is strongly stimulated. If this stimulation is accompanied by increase in permeability, as in the case of certain plant cells, and apparently in the case of the muscle cell, we should expect a reduction of the electrical polarization, increase in surface tension, and an approach toward the spherical form. 
That the surface of the ameba is electrically polarized is shown by the fact that the ameba moves toward the anode in an electric field, but this fact does not prove whether the electrical polarization is due to semipermeability or to the electrical polarization of the colloidal particles of which the surface of the ameba is composed. The movement in the electric field (electric convection) - may be abolished, or reversed, by adding $\mathrm{H}$ ions or certain heavy metal ions to the medium. These ions influence the permeability as well as the electric polarization of colloidal particles. The former is certain, and it is simplest to assume that the change in polarization is the result of the change in permeability alone, and not the combined effect of change in permeability and adsorption. The permeability hypothesis explains a number of physiological processes and is in harmony with a larger number of facts than any other.

All of those agents which stimulate muscle or nerve cause a rounding up of the ameba. We may interpret this approach to the spherical form as due to increase in the tension of the plasma membrane, following decrease in polarization, which is the result of increase in permeability or the disappearance of the semipermeability to certain ions or classes of ions. We should, therefore, expect most of the tropisms of the ameba to be negative, as is found to be the case. The ameba avoids the region from which a strong stimulus comes because the permeability and surface tension of the plasma membrane on that side is increased and the protoplasm flows in the opposite direction.

It is more difficult to explain positive tropisms, since decrease in permeability has been less often measured. But permeability being a relative term, decrease in permeability in one region has the same effect as increase in permeability in all other regions. Osterhout found that the permeability of kelp in calcium salt solutions is less than in sea water. This may be due to the fact that the sodium of the sea water increases the permeability and that the excess of $\mathrm{Ca}$ antagonizes the action of the sodium in the sea water, preventing this increase and making it appear that the calcium decreases it. We may assume that salts or other substances in the water tend to increase the permeability and that substances which antagonize their action cause positive tropisms. 
The action of an electric field on an ameba on a substratum is in harmony with this view. The anions within the ameba are massed on the inside of the plasma membrane toward the anode because they cannot pass through it. When they have accumutlated to a sufficient concentration they act as a stimulus, increasing its permeability, and the ameba moves toward the cathode. This is not due to electric convection, since a suspended ameba is carried toward the anode by this means, electric convection being anodic whereas galvanotropism is cathodic.

We may explain the irregular movements of the ameba when not under a single directive stimulus as due to varying and localized influences of the environment on its permeability, or to localized variations in its metabolism, resulting in variations in the production and concentration of the electrolyte responsible for the electrical polarization.

Certain substances dissolved in the medium increase and others decrease ameboid movements although the mechanism of their action is not known. Hamburger (I9I2) observed that anesthetics in certain low concentrations increase the ameboid motion of leucocytes. He found that a saturated solution of iodoform had the right concentration, and hence its efficacy in salves in increasing phagocytosis. This explanation rests on the assumption that the phagocytic action of the leucocytes varies with their ameboid activity. Substances which increase their phagocytic action were called opsonins by Wright, but whether such substances have their primary effect on the leucocytes or the bacteria seems undecided.

Ross (I9II) claims that alkaloids increase ameboid movements.

The idea that gelation and solation, and swelling and shrinking of gels are factors in ameboid movements was first expressed by Montgomery (I878, I88I), being more recently advanced by Rhumbler and others.

The contractile vacuole seems to be an organ of defense of the ameba against the swelling action of the hypotonic medium. Hartog has shown that it is universal among fresh water amebas but may be absent in parasitic and marine forms. It may be remarked, however, that even some phagocytes in exudates have contractile vacuoles. 
Rhumbler (I898) made a model of a contractile vacuole in the following manner. A drop of chloroform (not freed from alcohol, and that had become acid) was immersed under water. A clond of water droplets appeared within the chloroform and fused to one drop which burst.

The word tropism if used in strict accordance with its derivation would be confined to the multicellular and syncytial plants, but it has been extended to unicellular plants and to animals as well. For instance, the roots of plants are influenced by gravity. If moved out of the normal relation to gravity the tip bends back until the normal relation is regained. Starch grains in certain cells near the root tip are acted on by gravity and seem to act as otoliths stimulating the cells when the root is placed in an abnormal position.

Engelmann (I882) showed that the swarm spores of many green plants are influenced in their movements by external agents. He called these reactions to the environment tropisms, although the reaction is not a growing into a new position, but a turning movement or change in the direction of swimming.

Loeb (1889) has attempted to show that the tropisms of animals are the same as those of plants. This is largely a philosophical question, since the tropisms of plants are very imperfectly understood, and there is no consensus of opinion as to the dividing line between plants and animals.

Loeb distinguishes tropisms and sensibility to change, defining tropisms as the direct action of the environment on the motor organs, producing a direct and correct response. It should be added, however, that he also considers the reactions of certain insects to light as tropisms but cannot assume that the light acts directly on the muscles, the distinguishing characteristic being that the movements are forced. Even the behavior of vertebrates is sometimes described as tropism. Selachians do not seem to possess much intelligence since they butt their heads to pieces on the glass of aquaria until they die. All of their reactions are not simple, however. The author has often observed the rays of Mission Bay, San Diego, Cal., escape after being discovered. The ray moves along the bottom leaving a trail of muddy water, then doubles under this curtain and finally darts out at a new place. 
Jennings (I906) has shown that the movements of certain protozoa, that had been considered tropisms, are complex activities in which sensibility to change takes a leading part. Paramecium, when stimulated sufficiently by a change in the environment, reverses some of its cilia, so that it moves a short distance backward and the spiral course of its movement is widened. Being removed from the acute stimulation, it starts forward again from some segment of the spiral. The fact that the spiral is widened during the backward movement causes the course to be altered when the forward course is resumed. If this new course leads to a second stimulation the whole process is repeated. This repetition is continued until the animal ceases to be stimulated. Jennings calls this a case of "trial and error," thus showing a relation to the reactions of man. The distinction between tropism and trial and error is a philosophical one. There is no trial in the teleological sense and the cause of error will not be known until the mechanism of the reaction is understood. Trial and error has the same significance as chance in the laws of chance. Chance is merely a name to express ignorance of unknown factors. Only where these factors have some constant numerical relation to one another, is there a law of chance.

The question arises whether in Paramecium the stimulus acts directly on the motor organs, cilia. Since any strong stimulus, no matter from what direction, causes the same motor response, it would seem that there is some coordinating mechanism for the ciliary movement, or at least that the stimulus is propagated over the body of the Paramecium. Since the stimulus is propagated over the cells of sensitive plants without a special conducting mechanism, there is no reason to suppose that the same thing might not be true of animals. Nemec describes a conducting mechanism in plants, but this has been shown to be an artefact (McClendon, I9IO b). Neresheimer describes a conducting mechanism in infusoria, but this also is doubted.

The ameboid movements just described might properly be classed as tropisms, but even in the ameba there seems to be some evidence of the transmission of stimuli over the plasma membrane (McClendon, 1909 a).

The reactions of protozoa to constant electric currents seem to harmonize with the idea that tropisms are forced movements. 
Galvanotropism cannot be explained away as electric convection since the galvanotropism of the ameba is cathodic, whereas the electric convection of the ameba that is unable to reach and catch hold of the substratum is anodic. Ludloff (I895) showed that the galvanotropism of Paramecium is a forced movement, the cilia toward the cathode being reversed, and causing a turning toward the cathode. Since any strong stimulus causes reversal of cilia, this observation shows that the paramecium is stimulated at the cathode, even by a constant current. Dale (I9II) has studied galvanotropism in a number of species of infusoria.

The direction of tropisms has been reversed by change in viscosity, reaction, or temperature of the medium, and by the addition of neutral salts, acids and anesthetics. Some reagents employed, such as $\mathrm{CO}_{2}$ and alkaloids, change the reaction but their action may not be due to $\mathrm{H}$ ions. Wo. Ostwald supposes that a change in viscosity that reduces the efficiency of the motor organs changes negative geotropism into positive geotropism. If this is true, the head of the animal must already be pointed away from the earth, in order that positive geotropism take place. Furthermore, negative geotropism would be analogous to the effect of a strong constant electric current which carries the paramecium to the anode while its head is toward the cathode. Such a change from positive to negative geotropism is not what is usually meant by a reversal of tropism, which is made to imply a reversal of orientation: Some writers use the word taxis since it means orientation, but tropism, meaning a growing and borrowed from plant physiology, has come into general use.

Bancroft (1906) attempted to identify reversal of tropisms of Paramecium with the antagonistic actions of $\mathrm{Ca}$ to $\mathrm{Na}$, claiming that the direction of tropism is determined by the ratio of $\mathrm{Ca}$ to $\mathrm{Na}$.

We have attempted to explain ameboid motion, and consequently tropisms of the ameba as due to local increase in permeability. The ameba recedes from the side on which the permeability is increased. The agent causing positive tropism must therefore shield the ameba from stimuli. The movements of plants are caused by increase of permeability, and Tröndle (I9I0) and Lepeschkin (I908) have shown that light increases the permeability of plants to salts and dextrose. 
The geotropism of roots has been supposed to be due to the action of starch grains in the cells as statoliths. According to Czapek (1902) homogentisinic acid is produced in geotropic root tips and phototropic sprout tips, but the mechanism of its action is not clear.

Loeb has shown that phototropism obeys the Bunsen-Roscoe (I862) law. That is to say, the time required to induce a reaction is inversely proportional to the intensity of the light.

The perception of light is perhaps beyond the pale of physical chemistry at present. It should be noted, however, that many of the devices used in photography were first used in the construction of the eyes of animals. Even the "Lumiere" plate for color photography is represented by the retina of turtles and diurnal birds, which is covered with red and orange oil droplets. These are absent in the eyes of nocturnal birds (Winterstein, I9Io iv 577). The rods and cones beneath a red oil drop can be affected by red rays only and those beneath a yellow drop by yellow rays only, whereas those not covered by oil drops would be affected chiefly by the more effective blue rays. In this way a mechanism for color vision is provided, but whether it functions as such has not been determined.

One striking characteristic of the behavior of organisms and parts of organisms is their periodicity. The human body shows a diurnal periodicity that is perhaps most strikingly demonstrated in fluctuations in temperature, being coldest some time near 3 A. M. and warmest about to A. M. Each organ has its characteristic periodicity, respiratory center I6 and heart 72 per minute and the motor ganglia 50 per second. It is not always easy to determine what factors determine the period when it is very long. The Atlantic Palolo worm (Eunice fucata) comes out of its hole to liberate the genital segments before dawn on the last quarter of the moon between June 20 and July 25. The light or tide of the last quarter are unnecessary (A. G. Mayer). It is possibly the light of the full moon that prevents it from swarming too soon (some swarmed near full moon this year but another swarm occurred on the last quarter; it was not observed whether the full moon was overcast).

According to Bohn (I9I4) snails taken far from the seashore maintain a rhythm synchronous with the tides for a con- 
siderable period. This rhythm must be due, therefore, to metabolic changes, in the same way that the rhythm of the organs of the human body is maintained. Various physical models have been made showing periodicity, perhaps the most interesting of which are the periodic catalysis mechanisms of Bredig and his pupils. Under certain conditions mercury causes the decomposition of $\mathrm{H}_{2} \mathrm{O}_{2}$, and this in turn causes a film of oxide to form on the mercury. In these models the film breaks periodically and is reformed. If a crack appears in the film the potential difference between mercury and oxide dissolves the film electrolytically. The surface tension of the mercury differs from that of the oxide, so that surface tension movements as well as catalysis are produced (see Bredig, I907, B. and Fajans, I908, B. and Fiske, I9I2, B. and Weinmayr, I903).

\section{Cell Division}

Cell division in animal cells seems to be a special form of ameboid motion, and many cells show irregular ameboid movements immediately before division. It is thought by some investigators that the division of the nucleus is necessary before division of the cytoplasm can take place. On the contrary, it was found that the cytoplasm of cells from which the nuclei were removed is capable of division (McClendon, I908). The unfertilized starfish egg may be caused to divide by immersing it in sea water changed with $\mathrm{CO}_{3}$, as will be described later. The nuclei (maturation spindles) were removed from starfish eggs and immersed in carbonated sea water, developing imperfect mitotic figures (cytasters) and dividing into spherical masses of protoplasm. In the parthenogenetic frog's egg, to be described later, the cytoplasmic division commences an hour or more before the nuclear division commences, seeming to indicate that the nuclear division is not the direct cause of the cytoplasmic division.

Bütschli, Rhumbler and others considered cell division to be the result of changes in surface tension. The constriction of the cytoplasm follows a band of relatively high sutface tension. There seems to be no way of determining whether the surface tension is increased at the equator or reduced at the poles, but the division is often preceded by a rounding up of the cell, which is probably the result of a general increase in surface tension. 
That the surface tension at the equator is greater than at the poles of the dividing cell is demonstrated by the superficial movement of the protoplasm. When the tension of a surface is locally altered surface currents pass from the regions of low tension to regions of high tension. Gardiner ( 1897 ) observed the superficial granules in certain dividing eggs to pass in the direction from the poles to the equator. The superficial granules in the Arbacia egg become heaped up at the equator during cell division (McClendion, IgIo e), and must have moved in this direction, since they are evenly distributed before division commences. Conklin (I908) observed somewhat similar movements in the eggs of Crepidula.

It is difficult to make a model of cell division because it is difficult to maintain local clifferences in surface tension long enough for complete division to take place. The following model requires considerable skill to manipulate. A drop composed of one part chloroform and two parts rancid olive oil is immersed in water and prevented from touching the bottom by a layer of concentrated salt solution under it. Two pipettes filled with .I normal alkali are placed near opposite poles of the drop and the alkali allowed to flow onto these two poles simultaneously. The drop bulges at the poles and constricts in the middle. The reduction of the surface tension at the poles causes surface currents toward the equator, which spread the alkali over the whole surface of the drop. In some instances, however, division is completed before this is accomplished.

\section{Fertiliation}

Many egg cells do not divide unless they are fertilized by sperm, and it was formerly believed that they are incapable of division unless fertilized.

The process of fertilization may be divided into the following factors and stages: first, the finding of the egg by the sperm; second, the change in the egg responsible for cell division; third, the entrance of the spermatozoon into the egg and fusion with the female pronucleus; and fourth, the transmission of the paternal characters to the egg.

F. Lillie (1913) observed that the sperm of Nereis and Arbacia are attracted by substances given out by the eggs. Since he 
found that they are attracted by $\mathrm{CO}_{2}$ and this observation has been confirmed ( $\mathrm{McCl}$ endon, I9I4 e), it seems probable that it is the $\mathrm{CO}_{2}$ given out by the eggs that attracts the sperm, and hence it is not at all specific. Mammalian sperm appear to be attracted by cells of the wall of the uterus.

F. Lillie claims that the sperm cannot unite with the egg unless a substance which he calls fertilizin (a specific agglutinin?) is present to accomplish the union. When the sperm are treated with the agglutinin extracted from the ripe eggs of the same species, they become motionless with their heads adhering together. F. Lillie claims that these agglutinins are specific, but J. Gray (I9I5) has substituted hydroxyl ions or trivalent cations. Gray says that cerous chloride is as effective as the agglutinin extracted from the egg. He says further that $\mathrm{OH}$ ions and trivalent cations not only agglutinate but also activate the sperm, and that $\mathrm{H}$ ions, which reduce their activity, do not agglutinate. This inactivation by acids is reversible. F. Lillie had already stated that alkalis cause agglutination and also that acids cause aggregation. Since the attempts of Arrhenius and others to reduce immunochemistry to a physicochemical basis have been so hampered by lack of pure samples of the immune bodies, the discussion of the relation of fertilization to immunochemistry seems out of place at present.

The sperm does not need to penetrate the egg in order to cause cell division. Kupfelweiser, Bataillon (I9IO) and Brachet observed that the sperm needed merely to scratch the surface of the egg in order to make it segment. The sperm of foreign species usually cannot enter the egg, yet they may cause it to develop. This seems to indicate that the sperm causes a superficial change in the egg. Loeb called this a superficial cytolysis.

$R$. Lillie observed that some of the red pigment comes out of Arbacia eggs when they are treated with parthenogenic agents, and concluded that fertilization is accompanied by increase in permeability. Lyon and Shackell (I9IO b) showed that some of this pigment came out of the eggs on fertilization.

In order to test this conclusion, the electric conductivity of sea urchin eggs immediately before and after fertilization was measured (McClendon, I9Io c, e). The egg is surrounded by a mucous jelly and after fertilization a membrane, the fertiliza- 
tion membrane, appears between the jelly and the egg. Both of these might vitiate the conductivity experiments. It was found that if the jelly is washed from the eggs before they are fertilized, no fertilization membranes appear.

The eggs from which the jelly was washed (with sea water) were placed in the conductivity cell and gently centrifuged. The level to which the eggs came was marked on the cell and the conductivity determined by the Kohlrausch method. The eggs were then transferred to a large quantity of sea water with just enough sperm to fertilize all or at least 80 per cent of the eggs, then washed in fresh sea water and returned to the cell. The eggs were centrifuged down to the same level as before fertilization and the conductivity again determined, which was found to be greatly increased. This experiment was repeated many times with the same result. It was also confirmed by J. Gray (I9I3 a, b) who observed that about fifteen minutes after fertilization the conductivity began to fall again.

Since the spaces between the eggs were the same during the two determinations and were filled with sea water having the same conductivity in each determination, the change in conductivity is evidently due to the change in permeability of the eggs to ions.

This increase in permeability should decrease the polarization and increase the surface tension, supported by the fact that the eggs round up. The division of the eggs must be due to local changes in surface tension, and is therefore not the direct result of the initial permeability change, but probably an indirect result of it.

After the general increase in permeability, the original semipermeability is partially regained. It is only necessary to assume that the semipermeability is regained first at the poles, thus leaving the equator with the greatest surface tension, in order to account for the division:

The increase in permeability of the egg on fertilization was demonstrated in other ways. It was found that fertilized Arbacia eggs are more easily plasmolyzed by cane sugar solution than are unfertilized eggs, indicating permeability of the fertilized eggs to ions. This may be due, however, to increased permeability to water, observed by Lillie (1916 a). 
Lyon and Shackell (Igro b) observed that Arbacia eggs are more permeable to many dyes after fertilization. E. N. Harvey (I9II) observed that strong bases penetrate fertilized sea urchin egigs more easily than unfertilized. He seems to consider the alkali, however, as a permeability increasing agent to which the fertilized egg is the more susceptible.

The permeability of the frog's egg increases on fertilization (McClendon, I9I4 a, I9I5 a). These eggs will live and develop in distilled water. If any substances diffuse out of them, therefore, they may be detected in the water, and the permeability to the contained substances determined. The permeability of the egg to $\mathrm{Na}, \mathrm{K}, \mathrm{Mg}, \mathrm{Ca}, \mathrm{Cl}, \mathrm{SO}_{4}$, and $\mathrm{CO}_{3}$ is doubled by fertilization. Unfortunately it was not possible to measure the increase accurately in less than fifteen minutes in order to determine whether the original increase is partially reversed, as is the case with the sea urchin's egg.

According to Heilbrunn (I9I5) all initiation of development involves gelation of the substance of the egg. It is not understood how he can distinguish between gelation of the whole and gelation of parts, such as the membrane and the astral rays. It is well known that the astral rays are highly viscous (by centrifuge experiments) and a resistance of the egg to deformation before the appearance of the astral rays might be due to increased viscosity of the membrane.

The formation or separation of a fertilization membrane around the Echinoderm egg has given rise to much discussion. Heilbrunn says it is due to decreased surface tension. Loeb supposed it due to secretion of an osmotically active substance beneath the membrane. Glaser (I9I4) claims that the egg shrinks during the process which is further evidence of increase in permeability. An apparently similar process occurs in the frog's egg and Bialaszewicz (I908) has shown that the egg shrinks during the process.

\section{Artificial Parthenogenesis}

The eggs of some animals develop without the aid of a spermatozoon, a process known as parthenogenesis. Parthenogenetic development may be complete, as in case of the plant lice, or incomplete, as in case of the eggs of hens and silk worms, which if not fertilized, segment but die before development is complete. 
Hertwig and Morgan observed that the unfertilized sea urchin's egg may be caused to segment by treatment with certain solutions. Loeb continued these experiments with the object of obtaining complete development, calling it artificial parthenogenesis. Delage succeeded in raising several such sea urchin's beyond the larval stage. Bataillon reared several parthenogenetic frogs, and Loeb confirmed his experiment.

Loeb observed that cytolytic agents cause membrane formation on sea urchins' eggs, considering this due to a superficial cytolysis, also considering the development of the egg to be due to two factors, first, membrane formation, and second, a corrective factor which was not clearly defined.

R. Lillie supposed the development of the eggs to be due to increase in permeability. It was found that the electric conductivity of the sea urchin's egg increased when it was made parthenogenetic to the same degree that it did when it was fertilized (McClendon rgro c, e). The eggs were made parthenogenetic by treatment for a few seconds with a dilute solution of acetic acid in sea water, then being thoroughly washed in sea water. This increase in conductivity must be interpreted as an increase in permeability of the eggs to ions.

The permeability of the frog's egg increases when it is made parthenogenetic (McClendon, I9:14 a). It had been observed that the frog's egg will commence segmentation if a IIo volt alternating current is passed through the water containing the eggs from electrodes about two inches apart, for about one second (McClendon, I9I I b). This stimulation increases the permeability of the eggs to the same extent that fertilization does.

Since both fertilization and artificial stimulation act in the same way in increasing the permeability of the eggs, we have cumulative evidence to indicate that the increase in permeability is essential to segmentation.

The kinds of stimuli capable of inducing artificial parthenogenesis are very numerous. Heilbrunn (I9I3) claims that they all decrease the surface tension of the egg. It seems more probable, however, that the surface tension is increased, since the egg becomes spherical. It was found possible to cause the eggs of Arbacia punctulata to segment by treatment with any one of the following: hypotonic solutions, isotonic solutions of acids 
(especially fatty acids), alkalis or neutral salts, hypertonic solutions, mechanical shocks, thermal changes, electric currents, $\mathrm{KCN}$, lack of oxygen, or anesthetics (McClendon, I909 b). To these may be added in order to complete the list of parthenogenic agents, soap, bile salts, alkaloids, glucosides, blood sera, ultraviolet rays, and simply pricking with a needle. We thus have a list about as large as the list of stimuli for nerves. In what way all these agents increase the permeability is not known. The discussion of this question should be under the general topic of stimulation.

The question as to whether the fertilization membrane is present before fertilization has been much discussed. Harvey (I9IO a) says that it is not present, whereas Kite takes the opposite view. It has been supposed to be formed by the contact of the transparent gel covering the egg with a secretion poured out by the egg at fertilization, since it is absent from eggs that have been washed a long time, during which the gel is removed (McClendon; I9II a). Harvey supposes this washing injures the egg. The evidences for a secretion are numerous. The frog's egg, and according to Glaser (I9I4 b) the Echinoderm egg, gets smaller during this secretion. By the formation of this membrane it is merely supposed that a denser or more viscous layer is formed out of. material already present. It has been shown by microdissection that the surface of the unfertilized egg is not as viscous as the fertilization membrane (McClendon, I9I4 e). Heilbrunn (I9I5 a) supposes that the viscosity of the whole egg is increased by fertilization, but his proof that this increase in viscosity is not confined to the fertilization membrane is not olear. Loeb supposed the elevation of the membrane to be caused by the secretion of an osmotically active substance of high molecular weight behind it. Kite says the membrane swells and that we see the outer surface of it. It is probably pushed " out by the passage inward of water attracted by a colloid, but whether this is due to the osmotic pressure or swelling pressure of the colloid seems less important, as it is not certain that these two phenomena are different.

Many attempts to cause the eggs to segment with sperm extracts have been tried, but positive results have been attributed to impurities or lack of isotonicity (M. Morse, I9I2 a). Since 


\section{OF VITAL PHENOMENA}

the sperm contains lecithin and therefore fatty acid, and since fatty acids and many other organic substances cause membrane elevation and segmentation, it should be possible to cause segmentation with sperm extract. Robertson (I9I2) washed sperm in $\mathrm{H}_{2} \mathrm{O}$, precipitated the extract with $\mathrm{BaCl}_{2}$, redissolved it in $\mathrm{HCl}$ and reprecipitated it with acetone. The resulting "oocytin" caused membrane elevation. 


\section{CHAPTER XIV}

\section{MUSCULAR CONTRACTION, OXIDATION AND HEAT AND LIGHT PRODUCTION}

The muscle was at one time considered to be a machine in which a difference in temperature produced by oxidation is converted into mechanical energy, but this hypothesis does not harmonize at all with the facts. A. V. Hill (I9I3 a) has shown that when the muscle performs an isometric contraction in an atmosphere of nitrogen the heat produced is about half as great as when oxygen is present, and develops simultaneously with the contraction. Evidently this is a case, at least in part, of the transformation of mechanical work into heat. If oxygen is present, the heat produced is much greater, but nearly half of it is produced after the contraction is completed, evidently having nothing to do with the contraction.

\section{Striated Muscle}

The muscle may work for a considerable period without oxygen, or with the oxidations suppressed by $\mathrm{HCN}$. Weizsäcker (I9I2) reduced the respiration of frog's heart to 36 per cent of the normal for thirty minutes without reducing the work performed. By larger doses of $\mathrm{HCN}$ he completely prevented the consumption of oxygen, finding the work to be only reduced to about 70 per cent of the normal. This proves conclusively that the muscle is not a heat machine.

Hill measured the heat and work produced by a muscle with a relatively low tension and stimulus, and found the work to equal 9O-IOO per cent of the equivalent of the heat produced during contraction. The heat produced in the relaxation period was 80 per cent of that produced during contraction. Therefore the efficiency of the muscle is more than 50 per cent.

Engelmann (1893) showed that a catgut stretched in water would contract on addition of acid. Stretching of the catgut 
while swollen makes it anisotropic and if dried in this condition it will remain so until swollen again. The muscle fiber is anisotropic. Meigs ( $1908 \mathrm{~b}$ ) has shown that there are no isotropic discs, but merely, regions of less density or anisotropicity. Engelmann showed that the catgut would not contract on swelling unless anisotropic.

It has long been known that sarcolactic acid is produced by an active muscle, and increases in proportion to fatigue in a muscle in a nitrogen atmosphere. Fletcher and Hopkins ( I9o7) have recently reinvestigated this question. If oxygen is admitted to the fatigued musole in nitrogen, the lactic acid disappears, and $\mathrm{CO}_{2}$ and heat are produced. Some suppose the lactic acid to be the cause of the contraction. Death rigor has been compared to a long continued contraction, and is accompanied by lactic acid production. Winterstein (I897) has shown that an oxygen atmosphere may prevent the production of acid and likewise the death rigor in a dead muscle. Death rigor is apparently caused by a certain low concentration of acid, and when the acid increases above this the rigor disappears (Von Fürth and Lenk, I9II). This is in harmony with the observation of Spiro and others that low concentrations of acid cause swelling of hydrophile colloid gels, whereas higher concentrations do not. Von Fürth and Lenk (IgII) have shown that the muscle during death rigor and immediately before it, has a great power of absorbing water, but losing this power and even shrinking a little, due to loss of water, after the passing of the rigor. The shrinking is apparently due to the excess of acid. At a low acid concentration the muscle proteins swell, shrinking at a higher concentration.

McDougal (1908), Meigs (I908 a) and others have revived Engelmann's swelling theory of muscular contraction, with the substitution of osmotic pressure, as illustrated by the following model. A rubber tube has a series of close fitting metal rings slipped over it so that the distance between them is less than the diameter of the tube. The tube is closed at one end and water is pumped into the other. As the volume of the contained water is thus increased the length of the tube is decreased. The metal rings are supposed to take over the function of the Krauze's membranes of the muscle fiber. Later Meigs (I910) returned 
to Engelmann's idea that colloidal swelling is the cause of shortening.

Bernstein (IgOI) assumed that increase in surface tension caused the contraction of muscle. This hypothesis has the advantage that it relates the contraction to the electrical phenomena. As we previously noted, depolarization causes increase in surface tension. Ain increase in surface tension of the whole fiber, or even of the myofibrils, would have to be very great in order to account for the force of contraction of the muscle. Bernstein supposed the contractile elements to be minute ellipsoids, whose elasticity tended to maintain the elongated shape, whereas an increase of surface tension would make them more spherical.

Mathematical data may decide between these two theories. Bernstein (Igo8) points out that the temperature coefficient of muscular contraction as well as of surface tension is negative, whereas that of swelling of colloids is positive. $\mathrm{He}$ further shows (I9I5) that violin strings, such as used by Engelmann, are twisted, and if untwisted they do not contract on swelling.

$R$. Lillie supposes the surface tension increase to affect the colloidal particles in the muscle and cause their aggregation. This aggregation of colloidal particles is what is often expressed by the word coagulation. The experiments of von Fürth show that death rigor, at least, is not a coagulation but the reverse, a colloidal swelling.

\section{Smooth Muscle}

When smooth muscle contracts it remains so without fatigue and needs another stimulus (inhibitory nerve) to relax it. This is called the catch mechanism, and enables it to hold up a greater weight than an equal volume of striated muscle and yet without expenditure of energy. These facts together with the statement by Meigs (I9I2 c) that smooth muscle has no semipermeable membranes prevent the extension to it of the theory of muscle contraction just described.

\section{Oxidation}

In the enthusiasm following the discovery of enzymes, animal and plant oxidations were thought by some investigators to be explained by merely assuming that they were brought about by 
oxidases. With the search for oxidases many have been found and some of them seem to be specific. The fact, however, that no series of oxidases with which the complete oxidation of the ordinary foodstuffs can be accomplished have yet been isolated from the organized cell or cell fragment, places the question of oxidations in a separate field of research from enzymes in general. Harden and McLean (IgII) found that respiration did not occur in the press juice of muscle and some other tissues. Batelli and Stern (Igog) confirmed this observation but claim that respiration occurs in the press juice of liver. Warburg (I9I3) showed that most of the oxidation in liver juice is associated with granules. Whereas the oxidation of the liver juice is 20 per cent of that of the intact liver or liver cells, the oxidation of the juice after having passed a Berkefeld filter is only 4 per cent of that of the liver. As shown by Bechhold, such treatment may not remove all of the colloidal particles. Warburg found that the granular mass of ground sea urchin eggs absorbs oxygen but does not give out $\mathrm{CO}_{2}$. Warburg and Meyerhof (I9I4) found that the lecithin extracted from the egg when mixed with a ferric salt the equivalent of the iron in the egg, absorbed as much oxygen as the ground egg. This is probably due to the oxidation of unsaturated fatty acid radicals in the lecithin, and is accelerated by $\mathrm{H}$ ions. A similar reaction takes place in linseed oil and is accelerated by various metallic and organic oxides or peroxides. Warburg (I9I2 a) ground young erythrocytes (with rapid respiration) in a special ball mill (Macfadyen and Rowland) and the oxidation was reduced to zero.

Much of the earlier theoretical work on oxidations is based on the assumption that $\mathrm{O}_{2}$ is relatively inert and that nascent oxygen or some active oxide or peroxide is necessary to affect oxidation of difficultly oxidizable substances. This follows from the fact that oxidation consists in the addition of a positive charge or valence to the molecule. The molecule thus charged may combine with $\mathrm{OH}$, or if the charge is double it may combine with one atom of oxygen. What is meant by nascent oxygen is not clear. Perhaps some substance splits out $\mathrm{O}^{\prime \prime}$ from $\mathrm{O}_{2}$, thus leaving $\mathrm{O}^{*}$ which can exist only in a nascent state because it so easily parts with its positive charges, and in doing so oxidizes the substance receiving them. 
Various unsaturated compounds have been supposed to aid in oxidations by combining with one atom of the oxygen molecule thus leaving the other free to attack another substance that is not so easily oxidized. According to Bach and Chodat (r904) an oxidase is a mixture of two enzymes, oxygenase and peroxidase. The oxygenase combines with $\mathrm{O}_{2}$ to form a peroxide which is attacked by the peroxidase, with the liberation of active oxygen that in turn attacks the difficultly oxidizable stubstance present. $\mathrm{H}_{2} \mathrm{O}_{2}$ can be substituted for these peroxides. One criticism of the study of the oxidases is that relatively easily oxidizable substances have been selected with which to test their activity. It would be interesting to have a list of organic substances arranged in order of oxidizability, as well as a record of the oxidizing power of cell constituents of recognized purity if not known constitution. The author observed that oxyhemoglobin (recrystallized five times) as well as metahemoglobin (recrystallized seven times) would accelerate the oxidation of a-napthol, aloin and $\mathrm{p}$-phenylene diamine and an alkaline solution of a-napthol + $\mathrm{p}$-phenylene diamine, whereas hematin would not accelerate the oxidation of these substances without the addition of $\mathrm{H}_{2} \mathrm{O}_{2}$.

Since $\mathrm{H}_{2} \mathrm{O}_{2}$ or some other peroxide is necessary for the activation of the peroxidases of the cell, all cells contain catalase which decomposes $\mathrm{H}_{2} \mathrm{O}_{2}$ with enormous rapidity, with the liberation of inactive oxygen. It has been suggested that catalase protects the protoplasm against oxidation. But how is the oxidation regulated so as to occur at the right place at the right time? The answer to this seems to lie in cell structure, which will now be considered.

It was stated in the chapters on the bioelectric phenomena and muscular contraction that when a muscle contracts dextrose disappears, the permeability of the cells is increased, lactic acid appears between them, if oxygen is admitted the lactic acid disappears, and $\mathrm{CO}_{2}$ and heat are produced. The simplest explanation of this chain of phenomena is that in the muscle fiber lactic acid is produced until the reaction is stopped by the accumulation of this end product. This would make the reaction of the protoplasm slightly acid, but it need not even reach the isoelectric point of the proteins, so that the proteins may continue to be electronegative. That the cell sap of certain plant cells is acid 
is shown by their red color, and indicators swallowed by protozoa have shown an acid reaction of the vacuole contents. But it is impossible to determine the reaction of the protoplasm itself, except to say that it is less acid than the isoelectric point of the proteins or lipoproteins of which it is formed. As soon as the lactic acid reaches the outer surface of the plasma membrane it is burned, provided $\mathrm{O}_{2}$ is present. As will be shown later, the oxidase concerned is probably associated with the plasma membrane, possibly adsorbed to its outer surface. Anesthetics might suppress oxidation in two ways, by preventing the permeability increase, or by coating the oxidase with a layer preventing the access of the oxidizable substance.

The evidence that such a process might take place in other cells is found in the fact that the oxidation of the sea urchin's egg increases sevenfold when its permeability increases, and Warburg (IgI I b) has shown that the oxidation of erythrocytes is increased on laking by freezing and thawing (provided there is not too much serum or salt solution present). Warburg gives as evidence of surface action the fact that alkaline sea water or pure $\mathrm{NaCl}$ solution increases the oxidation of unfertilized sea urchin eggs sevenfold whereas they do not penetrate the protoplasm. He takes experiments of Overton as evidence that $\mathrm{NaCl}$ does not penetrate, and as evidence that $\mathrm{NaOH}$ does not penetrate the fact that this concentration of $\mathrm{NaOH}$ does not turn eggs yellow that have been stained with neutral red, whereas $\mathrm{NH}_{4} \mathrm{OH}$ of less alkalinity does.

Colorless substances may be oxidized by cells to colored compounds that appear as granules in the cell interiors. This does not prove that oxidation occurred in the exact spot in which the granules are seen. Certain granules in cells absorb or adsorb and concentrate dyes so that they may remove all of the dye from their surroundings. Even though an oxidation product is said to be insoluble, solubility is a relative term, and the diffusion of an extremely dilute solution of a colored substance cannot be detected with the microscope because this instrument does not make the color appear more intense than it actually is. But it does not seem reasonable that all of the oxidation takes place on the cell surface. Warburg (I9II b) has shown that minute quantities of $\mathrm{NH}_{3}$, insufficient to change the reaction of the 
serum to indicators, may increase the oxidation of erythrocytes, whereas $\mathrm{NaOH}$ is ineffective. Since $\mathrm{NH}_{3}$ penetrates so much more easily than fixed alkalis, this indicates that $\mathrm{NH}_{3}$ affects oxidation in the interior, or else the action is on the plasma membrane.

Warburg found that anesthetics reduced the oxidation of erythrocytes according to their anesthetic power. He illustrated it by the following model (I9I4): Blood charcoal causes the oxidation of oxalic acid adsorbed to its surface to $\mathrm{CO}_{2}$ and $\mathrm{H}_{2} \mathrm{O}$. This charcoal, even after washing with $\mathrm{HCl}$, contains iron which may be the real catalyzer. The catalytic action of the charcoal varies with its power of adsorbing oxalic acid. If an anesthetic is added to the solution of oxalic acid the oxidation is reduced. The more easily the anesthetic is adsorbed the greater is its inhibitory action. The explanation of this action is that the anesthetic is more strongly adsorbed than the oxalic acid, which it displaces from the surface of the charcoal.

McClendon and Mitchell (19I2) showed that those solutions that were most effective in increasing the permeability and causing the segmentation of unfertilized sea urchin eggs, are also most effective in increasing the oxidation. Warburg (I9Io a) combats the idea that the segmentation is the cause of the oxidation. He finds that phenyl urethane may stop the segmentation without reducing the oxidation to a significant degree. But the segmentation and increased oxidation might be due to increased permeability without the two running parallel. Let us suppose that oxidation is proportional to permeability. A too great increase in permeability will prevent segmentation, but it might greatly increase oxidation.

Whereas Warburg is forced to admit some connection between the plasma membrane and oxidation, he claims this does not hold true for the unfertilized sea urchin egg, whose oxidation is not decreased by grinding with sand. But this fact is capable of another interpretation. It may be a mere coincidence that the absorption of oxygen by the intact and the ground egg is the same, since the production of $\mathrm{CO}_{2}$ ceases on grinding. The first impact of the sand may cause the eggs to begin development, since they may be caused to segment by shaking in a vial. In this condition they are the same as fertilized eggs, with enor- 
mously increased oxidation. This view is supported by the fact that Warburg found the oxidation of unfertilized eggs burst in distilled water to be temporarily increased. They may be caused to develop by a short treatment with distilled water or longer treatment with hypotonic solution. After the egg is ground with sand the oxidation falls to the same level as a fertilized egg ground with sand, and in neither case is $\mathrm{CO}_{2}$ given out. The $\mathrm{CO}_{2}$ output of the unfertilized egg is totally changed (abolished) by grinding and the fact that the $\mathrm{O}_{2}$ absorption is the same at a certain time after grinding may be a mere coincidence.

Warburg and Wiesel (I9I2) have attempted to show the similarity between the oxidizing power of cells and enzyme action, in the reduction of oxidation and fermentation of yeast to the same degree by anesthetics. The activity of the zymase in yeast press juice is but a small fraction of the activity of the yeast, but the oxidation has been reduced to nil. There is a slight oxidation in the filtered liver press juice, however, and this may be compared to the zymase in yeast juice. The significance of cell structure, however, is shown by the fact that the oxidation of various cells is reduced to about one-third by killing the acetone, which leaves part of the structure intact.

The results of Batelli and Stern (I9I4) also indicate a relation between cell structure and oxidation, but they probably interpret it differently. They divide respiration into chief or rapid and accessory or lasting respiration. This accessory respiration seems to be due to cell fragments, dead cells, cell granules (and bacteria?) and only a small percentage of cell juice proper. They also studied the oxidizing power of ground tisstues on various substances and conclude that the citric acid oxidase ("oxidone") is destroyed with cell structure, but the others are not. They attempted to get more oxidases in the extract by grinding the tissue finer, using a Borrel mill with revolving knives, but this treatment merely reduced the oxidation of the cell fragments, without increasing the oxidation of the extract.

Warbung observed that the oxidations that take place in cell extracts may be reduced by anesthetics, but the concentration of anesthetic required is many times that required to reduce the oxidation of the intact cell a corresponding amount. $\mathrm{He}$ explained this as due to the fact that the oxidase in the cell is ad- 
sorbed to a surface, the anesthetic adsorbed to the same surface, and its concentration increased above that in the interior of the solution. Meyerhof (Igr4 a) attempted to make a model to show this difference. He found that the activity of an invertase solution is not altered by adsorption to colloidal ferric hydrate (dyalized iron). If the same concentration of anesthetic is added to the invertase solution and the invertase iron suspension, the activity of the latter is reduced more than the former, but the difference is very small, not nearly so great as between the invertase inside and outside the cell.

The question as to whether the cells of the body would use more oxygen if they were more liberally supplied with it is very important. Paul Bert ( 1878 ) found that animals died in oxygen under pressure, but this phenomenon has never been thoroughly analyzed. Anaerobes and facultative anaerobes may live indefinitely and many other organisms may live for a time without oxygen. Infusoria may live from five to twenty days without oxygen (Putter, I9I I). The development of Fundulus eggs may be suspended by withholding oxygen and commence again when it is readmitted. Henze (I9ro) found that sea anemones use more oxygen the greater its concentration in the sea water. When protein is eaten the mammal uses more oxygen than when a carbohydrate of the same calorific value is eaten. One explanation of this might be that the protein gives rise to acid substances in the blood which stimulate the respiratory center and cause more oxygen to be brought to the tissues.

\section{Heat Production}

The heat produced by burning fats and carbohydrates in the body is the same as that obtained in burning them in the bomb calorimeter, 3.74 Cal. per gram of monosaccharide, 3.95 for disaccharide and about 9.5 for ordinary animal or vegetable fat. But the heat produced by protein is 5.7 in the calorimeter and only 4.5 in the body. The difference is that the protein is not completely burned in the body, being excreted as urea, uric acid, $\mathrm{NH}_{3}$ and other incompletely oxidized products.

While heat production and loss in man is so regulated from the heat center in the brain that the temperature of the body, to within an inch of the surface, seldom varies more than a de- 
gree during health, this is not at all the case with the lower organisms. The temperature of small marine animals is never more than $.7^{\circ}$ above that of the medium. The body of an isolated insect may be $4^{\circ}$ above that of the air, whereas the temperature in the center of a swarm of bees may be $17^{\circ}$ above that of the air.

\section{Light Production}

Light is produced by bacteria, fungi, protozoa, medusae, ctenophores, molluscs, fish, crustacea and insects, and the eggs and larvae of photogenic animals. The sea on a favorable night reveals a host of wonderful luminous creatures. The effect of myriads of luminous Dinoflagellates in the Pacific is very striking. The water is reddened by day and turned to a sea of fire at night by these protozoa. Spray, the crests of waves and the outlines of fish and bathers are illuminated. The species is Gonyaulax polyedra.

In all organisms which produce light intermittently, the production of light follows stimulation with all classes of stimuli. If the production of light is always due to oxidation, it forms a convenient index of the control of oxidation by the organism or cell. When the photogenic organism is crushed, the light is emitted at a rapid rate for a short time. An analogy to this luminescence of the dead organism is seen in "chemiluminescence" which will now be described.

It was shown by Callaud and Pelletier that wax, fatty oils, sugar and alkaloids, emit light on being warmed. Radziszewski (I883) showed that these substances even if kept in the dark, and not previously exposed to light, emit light on oxidation with alkaline solutions containing $\mathrm{O}_{2}$. Besides glucose, gallic acid, etherial oils, cholesterin and lophin, he found that unsaturated fatty acids and compounds containing them may emit light. His list includes oleic, elaidic and ricinoleic acids and their fats and soaps and lecithin, protagon and cetyl alcohol wax.

It has been pretty clearly established that the production of light by animals and bacteria is dependent on oxidation. Recently E. N. Harvey (19I4) has dried the photogenic organs of the firefly in vacuo and found that it could be kept in this condition indefinitely and at any time caused to emit light by the addition of water containing $\mathrm{O}_{2}$. Harvey (I9I6) obtained from. 
Japanese animals luminous secretions and extracts that are free from particles. According to Raphael Dubois (I9I3), these secretions contain "luciferase" which can be replaced by potassium permanganate and "luciferin," of unknown chemical nature. Harvey (I9I6) finds that various oxidases may be substituted for luciferase.

Coblentz (I912) photographed the spectrum of the light emitted by two species of firefly of the genus Photinus. The light contains only from yellowish green to orange rays. It is a cold light and is also lacking in rays of short wave length. He found the temperature of the luminous organ to be only a fraction of a degree above that of the same organ when not emitting light. It is probable but not certain that this heat is produced by the oxidation of the photogenic substance, as the admission of oxygen to the organ would naturally result in cell respiration.

Note: In Abderhalden's table, p. I75, for $\mathrm{Na}$ read $\mathrm{Na}_{2} \mathrm{O}$; for $\mathrm{K}$ read $\mathrm{K}_{2} \mathrm{O}$; for $\mathrm{H}_{2} \mathrm{PO}_{4}$ read $\mathrm{P}_{2} \mathrm{O}_{4}$. 


\section{CHAPTER XV \\ BLOOD AND OTHER CELL MEDIA}

The coagulation of blood is nothing more than a gel formation of a protein hydrosol, the gel being called fibrin and the same substance in colloidal solution, fibrinogen. According to Howell (19I4) the fibrin clot is a mass of acicular crystals. This coagulation leads to the distinction of plasma, the medium in which the blood corpuscles float, and serum, the same minus the fibrinogen.

As was previously stated, the permeability of the blood corpuscles seems to be nearly the same as the permeability of plant cells. Overton claimed plant cells to be impermeable to salts. The same seems to be true of blood corpuscles for otherwise how is the difference between the salts of the plasma and serum maintained since Höber has shown that the ions are not bound but free to move within the corpuscles. Abderhalden gives the following data of the salts of corpuscles and plasma in parts per hundred:

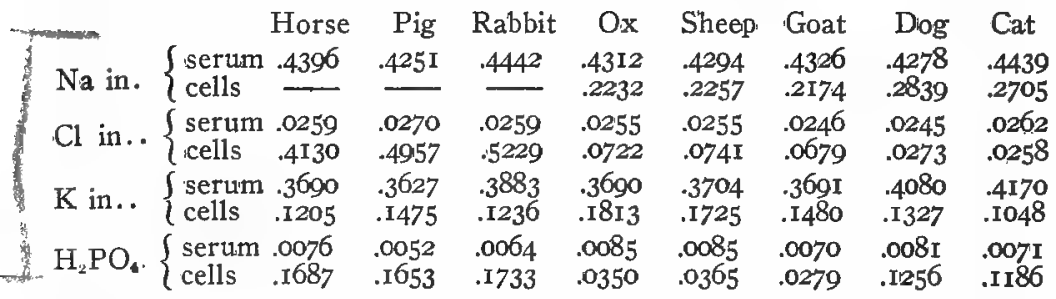

Notwithstanding this evidence Hamburger claims that the corpuscles are freely permeable.

The main difference noted between the permeability of plant cells and blood corpuscles was in case of urea, to which the plant cell is said to be impermeable, whereas some blood corpuscles, at least, allow it to pass through. It would be interesting to test the permeability of the erythrocytes of elesmobranchs (sharks and rays) to urea, since its concentration in the blood of these fish is about 5 per cent, whereas it exists only in very small quantities in the blood of other animals. 
The osmotic pressure of the blood of warm blooded animals is about eight atmospheres, about as constant as the body temperature. As we descend in the animal kingdom the osmotic pressure of the blood is more and more influenced by the water the animal drinks or lives in, reptiles, amphibians, bony fish, and all air and fresh water animals, being more or less independent of the medium. The osmotic pressure of the blood of all other marine animals is the same as that of the sea in which they live. The following table gives the freezing point lowering of the blood (or of some fluid approximately isotonic with the blood) of a number of animals:

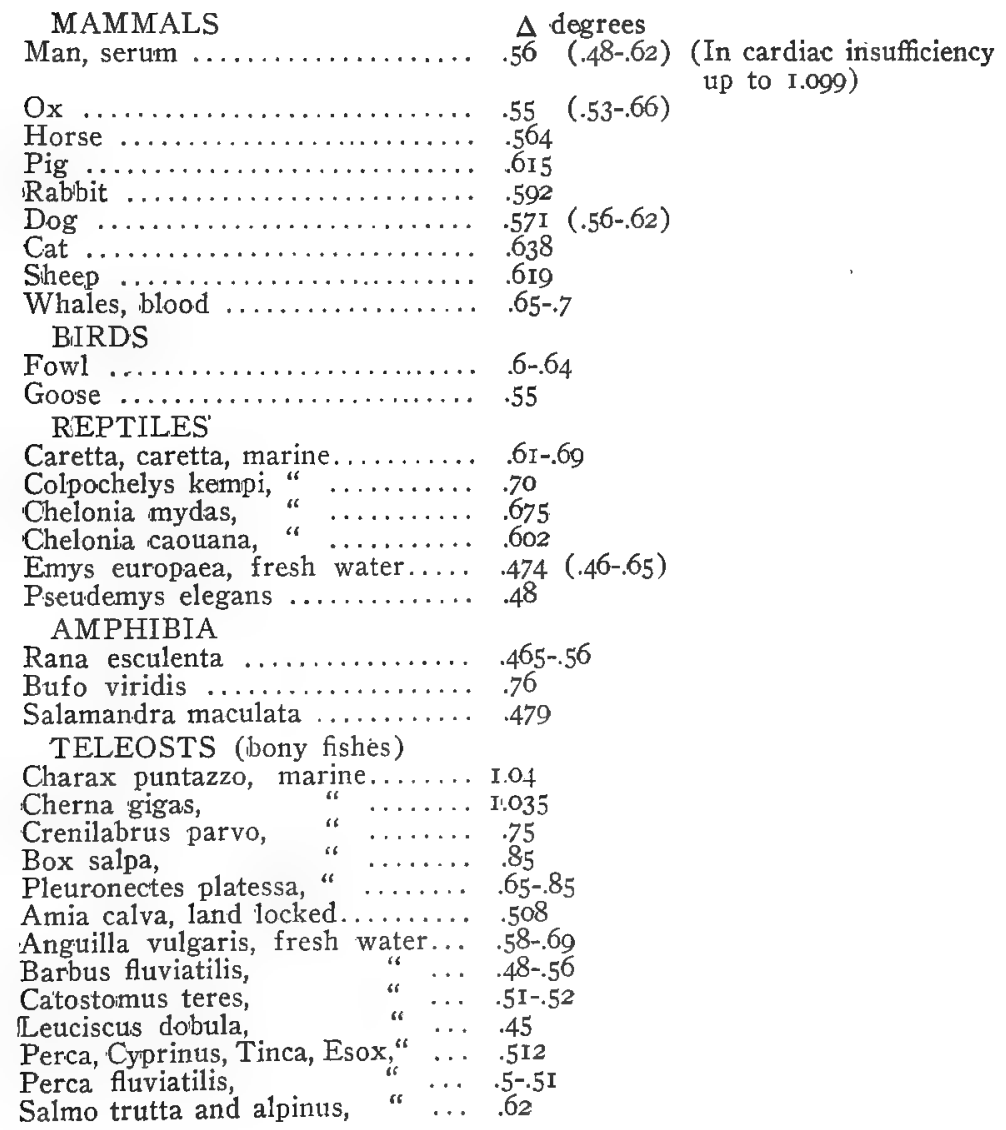


GANIOIDS

Acipenser sturio ............... $\quad .76$

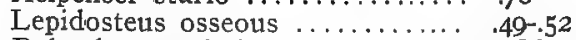

Polyodon spathula $\ldots \ldots \ldots \ldots \ldots, . . .486-.50$

INVERTEBRATES (excepting marine) (blood + body juices)

Anodonta cygnea $\ldots \ldots \ldots \ldots \ldots, .2$

Astacus fluviatilis $\ldots \ldots \ldots \ldots \ldots, 8$

Dytiscus marginalis ............. .57

Hirudo medicinalis ............. $4.4-43$

Dragon flies ................ .65

The osmotic pressure of the blood plasma must be imitated in any solution used to wash the corpuscles, as otherwise cytolysis (hemolysis) may result. If $\mathrm{NaCl}$ solutions are desired, .95 per cent is used for mammals, reptiles and birds and .65 per cent for frogs. A I per cent $\mathrm{CaCl}_{2}$ (anhydrous) solution, or .74 per cent $\mathrm{KCl}$, or .75 per cent $\mathrm{NaCl}$ has a $\Delta=.465$. In order to reduce the toxicity of the $\mathrm{NaCl}$, the solutions described at the end of the chapter are used. If cane sugar is used where a non-electrolyte is desired, about ten times as much is weighed out as in case of $\mathrm{NaCl}$ for an equal volume of solution (see Fig. 30). Sugar solutions are slightly toxic, probably on account of the $\mathrm{H}$ ions the sugar dissociates. An absolute non-electrolyte has not been found. It is possible that a small proportion of urea added to the sugar solution might reduce the $\mathrm{H}$ ion concentration sufficiently.

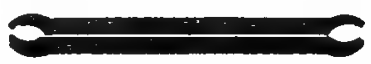

FIG. 29. Viscosimeter for a drop of blood. A little hirudin or oxalate is placed in one bulb, which is filled with blood. The tube is grasped with forceps and inverted. The viscosity is proportional to the time required for the blood to leave the bulb.

The blood in passing through the capillaries of the body undergoes many changes, not all of which are well understood. The effect of $\mathrm{CO}_{2}$ on the blood is more complicated than was at first supposed. When $\mathrm{CO}_{2}$ is bubbled through blood the $\mathrm{H}$ ion concentration is increased in the plasma and the $\mathrm{OH}$ ion concentration reduced, but, contrary to what might be expected, the titratable alkalinity is increased. This is partly explained by the fact that the $\mathrm{Cl}$ ions are reduced by the substitution of carbonates for chlorides. Koeppe ( 1897 ) put forward the following hypothesis: The $\mathrm{CO}_{2}$ enters the corpuscles and combines with the 


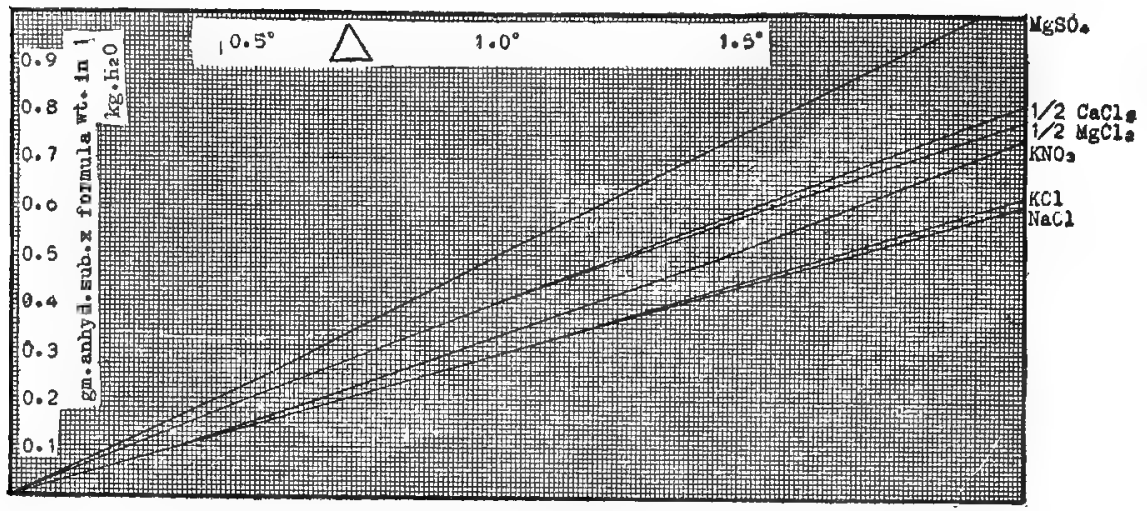

FIG. 30. Freezing point lowering of weight normal solutions. The scale is too small to show the increased ionization at very great dilution. The curve for $\mathrm{MgSO}_{4}$ approximates that for cane sugar and dextrose and is near that for $I / 2 \mathrm{~K}_{2} \mathrm{SO}_{4}$. The curve for $\mathrm{KCl}$ may be used for $\mathrm{KOH}, \mathrm{NaOH}$ and $\mathrm{NaNO}_{3}$. The curve for $\mathrm{NaCl}$ approximates that for $\mathrm{NH}_{4} \mathrm{Cl}$ and is near that for $\mathrm{Na}_{2} \mathrm{SO}_{4}$ for the higher concentrations.

proteids. This combination dissociates to a greater extent than $\mathrm{CO}_{2}$ and the excess of $\mathrm{HCO}_{3}$ ions so produced diffuse into the plasma, leaving the interior of the corpuscle electropositive. The $\mathrm{Cl}$ ions of the plasma are attracted by this electropositive charge and enter the corpuscle. Only a small part of the $\mathrm{CO}_{2}$ added takes part in this exchange, however, and a larger proportion of it is stored in the compuscles than in the plasma. This follows from von Limbeck's observation that the osmotic pressure of the corpuscles increases more than that of the plasma, causing the corpuscles to swell, due perhaps to the fact that alkali within the corpuscles binds the $\mathrm{CO}_{2}$. This alkali is supposed by some investigators to be ordinarily combined with protein, but in the presence of an excess of $\mathrm{CO}_{2}$, joins the latter.

This experiment shows that, at least in the presence of an excess of $\mathrm{CO}_{2}$, the erythrocytes are permeable to ' $\mathrm{Cl}$ ions. Hamburger and van Lier (1902) have shown by the same method that the erythrocytes are permeable to $\mathrm{SO}_{4}$ and $\mathrm{NO}_{3}$ ions.

Henderson and Spiro (I908) made a model of this process. A beaker of $\mathrm{Na}_{2} \mathrm{CO}_{3}$ solution represented the plasma, a parchment paper tube filled with $\mathrm{Na}_{2} \mathrm{CO}_{3}+$ protein representing a 
corpuscle. $\mathrm{CO}_{2}$ is bubbled through, the titratable alkalinity of the pure $\mathrm{Na}_{2} \mathrm{CO}_{3}$ solution is found to be increased because $\mathrm{Na}$ Protein is changed to $\mathrm{H}$-Protein $+\mathrm{NaHCO}_{3}$ and the latter diffuses into the pure solution. As is readily seen, the analogy is not exact.

It is possible that the plasma membrane of the erythrocyte is different from that of other cells. The stroma of these cells contain a large percentage of lecithin and cholesterin. Pascucci (1907) made membranes of lecithin and cholesterin that could be laked with the same reagents that lake corpuscles. These membranes were more easily laked the greater the percentage of lecithin, the same being true of compuscles. Perhaps some laking agents combine with lecithin. Berczeller (19I4) found that the surface tension of alkaline solutions of lecithin is increased by the addition of chloroform or ether. The relation of lecithin and cholesterin in the serum to certain hemolysins has been much studied, lecithin favoring and cholesterin inhibiting hemolysis. According to von Dungern and Coca (I908 a, b, c) the hemoly$\sin$ in snake venom is a lipase that splits off fatty acid from the lecithin, and the latter hemolyzes the corpuscles. Iscovesco (I9IO c) claims that cholesterin inhibits the action of lecithin by combining with it. Lecithin reduces the surface tension of water, but on addition of cholesterin it rises again. He claims that cholesterin acts on soap in the same manner.

Though anesthetics in high concentration are hemolytic, Traube (I908 a) found that small doses inhibit hemolysis. He claims that hemoglobin, itself, when in the serum, is hemolytic, but his specimen of hemoglobin was apparently impure.

The relative volume of corpuscles and plasma may be determined with the hematocrit (Hedin) or calculated from the conductivity (Stewart) volume of corpuscles

$$
=92 \frac{\text { conductivity of blood }}{\text { conductivity of serum }}+\mathrm{I}_{3} \text {. }
$$

The hematocrit was used by Hamburger to find the osmotic pressure on the assumption that the corpuscles are impermeable, although, in a series of later papers, he claims that they are freely permeable.

The viscosity of the blood is determined by the total volume 
of the corpuscles and to a less extent by the proteins of the plasma. The normal viscosity is $4.05-6.8$, is greater in man than in woman, and increases after feeding and after muscular work. In cardiac insufficiency it may reach 8.1 and in polycythemia 14.4-23.8. It is also increased by stasis. A convenient viscosimeter for blood is shown in Fig. 29. (It may be obtained from A. S. Jones, Princeton, N. J.)

The fact that the viscosity of the blood is determined chiefly by the total volume of the corpuscles suggests a method of determining the corpuscular volume. Such measurements must be made at nearly the same temperature, to be comparable. The viscosimeter is standardized with $\mathrm{H}_{2} \mathrm{O}$ at the temperature at which the blood is investigated, but the absolute viscosity of water is 27 per cent less at $37^{\circ}$ than at $I 7^{\circ}$ whereas this ratio is not the same for blood (blood is said to be 16 per cent less viscous at $37^{\circ}$ than at $I 7^{\circ}$ but it is not clear that the absolute viscosity is meant). According to Hess the hemoglobin may be calculated as follows: hemoglobin

viscosity

content of the corpuscles or the plasma proteins or the proportion of leucocytes is changed.-

The normal refractive index of the serum of man is I.345-I.35. According to Pearl (I9I4) the refractive index of the serum of the fowl is 1.34537 and of the guinea hen I.34I84.

It is usual to consider the solvent power of the blood plasma as being nearly the same as that of water, but this is not always the case. Uric acid is practically insoluble in water, the concentration of its saturated solution at $18^{\circ}$ being $.000 \mathrm{I} 5 \mathrm{~mol}$, the molecular weight being r68.2. Bechhold and Ziegler (I9Io) found that a liter of serum would dissolve more than half a gram of uric acid, at $37^{\circ}$, showing that its solubility in serum is enormoursly greater than in water. The reason for this difference is not self evident. Uric acid is a weak acid, in saturated solution in water being only 9.5 per cent dissociated. Although theoretically a dibasic acid it acts like a monobasic acid, as only one $\mathrm{H}$ ion is dissociated ordinarily. The primary sodium salt is more soluble than the free acid in water because it is strongly dissociated. According to Gudzent (I909 a) there are two isomeres, 
a saturated solution at $37^{\circ}$ of the laktam form being or mol, and of the laktim form being .0068 mol. The solubility is greatly reduced by the addition of $\mathrm{Na}$ salts, which accounts for the fact that it is more than thirty times as soluble in water as in physiological salt solution. The primary lithium salt is much more soluble. This has led to the practice of giving lithia water to gouty patients, but it is impossible to decrease the concentration of $\mathrm{Na}^{\circ}$ in the blood to the extent that the solubility of uric acid would be noticeably increased.

Since uric acid, when added to serum, becomes primary sodium urate, and the solubility of this is very low, it seems strange that the solubility of uric acid in serum should be relatively higher than the urate. According to Schade and Boden (Igr3), however, the uric acid forms a colloidal solution in serum, and in water, also, under certain conditions. If uric acid is dissolved in water by means of heat and the addition of minute quantities of alkali, a 2 per cent solution may finally be obtained. By the addition of neutral salts or by cooling, the solution may be transformed into a transparent gel, which after a greater or less time deposits crystals of uric acid. It is probable that the colloidal solution in serum is protected from crystallization by the other colloids present (protective colloids).

The blood plasma is very slightly alkaline, this alkalinity being maintained by the bicarbonates (and only to slight extent by phosphates) it contains. Höber ( $1903 \mathrm{~b}$ ) was the first to measure the $\mathrm{H}$ ion concentration of the blood. The later work on this subject has been largely concerned with the perfection of the technique. It has been shown that the PH of the blood is hardly affected by the changes in temperature to which it is ordinarily subjected (McClendon and Magoon, I9I6) and, therefore, the determinations of the $\mathrm{PH}$ of the blood at different temperatures by different observers would be comparable, provided the proper precautions were taken to prevent loss of $\mathrm{CO}_{2}$. The technique was described in Chapter IV, 7.5 taken as about the average $\mathrm{PH}$ of human blood. The $\mathrm{PH}$ of the blood of different individuals is so constant as to come almost within the limit of error of measurement. Hasselbalch and Gammeltoft found the PH of the blood of a number of supposedly normal individuals to vary from 7.4 to 7.49 . Michaelis (1914) found 
the $\mathrm{PH}$ of normal human blood, dropped through the air into a relatively large volume of $\mathrm{CO}_{2}$-free salt solution, to vary from 7.49 to 7.64 , but these high (alkaline) values are probably due to faulty technique. Winterstein (I9II) showed that if new born pups are perfused with $\mathrm{CO}_{2}$-free saline, the respiratory movements are not induced, but if a trace of $\mathrm{HCl}$ is added to the perfusing fluid, the respiration commences. It was shown by Hasselbalch and Lundsgaard (I9r2) that the respiratory center in anesthetized dogs is controlled by the $\mathrm{H}$ ion concentration of the blood. Milroy (I9r4) obtained similar results on dogs and cats but claimed that the threshold of stimulation of the respiratory center by $\mathrm{H}$ ions is lowered by lack of oxygen. This apparent result of his experiments may be explained away in the following manner: We may suppose that the respiratory center is affected not by the reaction of the blood directly, but by its own reaction, which is in a dynamic equilibrium with that of the blood (by diffusion). During lack of oxygen acid substances produced in the respiratory center fail to be removed by oxidation, and hence the $\mathrm{H}$ ion concentration increases although that of the blood remains constant.

In the same way the lowering of the buffer value of the blood in acidosis may affect the respiratory center. The lack of bicarbonate in the blood coming to the respiratory center decreases its power of neutralizing the $\mathrm{CO}_{2}$ produced by the center, and increased breathing may result. This may cause a decrease in the $\mathrm{H}$ ion concentration of the blood and decreased breathing. In this way irregular or labored breathing may arise, the total lung ventilation being increased owing to the low $\mathrm{CO}_{2}$ tension of the blood and alveolar air. That is to say, a given volume of expired air would carry a subnormal amount of $\mathrm{CO}_{2}$, and, hence, the lung ventilation must be increased in order to remove the $\mathrm{CO}_{2}$ from the body at the normal rate. The decreased buffer value of the blood with lowered $\mathrm{CO}_{2}$ tension is shown in fig. I8. Szili (1906) slowly injected $\mathrm{HCl}$ into the blood of dogs until it caused death. The PH of the blood of the dog at death was about 7.05 .

Owing to the prompt regulatory action of the respiratory center, the addition of acid within certain limits to the blood lowers its buffer value or bicarbonate content, and hence lowers the $\mathrm{CO}_{2}$ 
tension of the alveolar air. Since more exact data on the alveolar air are available we may take them as the most reliable index of the changes in the buffer value of the blood. Barcroft (19I4) measured the $\mathrm{CO}_{2}$ tension of the alveolar air, but put most reliance on the $\mathrm{H}$ ion concentration of the blood at a certain $\mathrm{CO}_{2}$ tension. In this way the buffer value is indicated as shown in Fig. I8. Barcroft estimated the $\mathrm{H}$ ion concentration of the blood by its effect on the dissociation of oxyhemoglobin.

Hasselbalch and Gammeltoft (I9I5) observed a lowering of the $\mathrm{CO}_{2}$ tension on a meat diet. This suggests an explanation of the so-called dynamic action of protein (Lusk, I9I5). On passing from a protein poor to a protein rich diet the heat output of the body is increased, even though the available energy of the food is not. Since the $\mathrm{CO}_{2}$ tension of the alveolar air is decreased, the lung ventilation must be increased and the oxygen carried to the tissues increased as well. Perhaps the greater heat production is the direct result of this increased oxygen supply. If this be true, we may suppose that the amino acids arising from digestion of the protein increase the $\mathrm{H}$ ion concentration of the blood, and stimulate the respiratory center, increasing lung ventilation, this in turn increasing oxidation. Since the respiratory center is more sensitive to changes in $\mathrm{H}$ ion concentration than any apparatus available for measuring it, a failure to determine any increase in the $\mathrm{H}$ ion concentration of the blood is to be expected. It was shown by Haldane and Priestley (1905) that a 0.2 per cent rise in the $\mathrm{CO}_{2}$ tension of the alveolar air doubles lung ventilation. A reference to Fig. 18 shows that this causes only a minute change in the $\mathrm{PH}$ (a change too small to measure with certainty on a carbonated fluid like the blood).

We may conclude from the foregoing that the buffer value of the blood is lowered by a meat diet. The same seems to be produced during exercise, acclimatization to high altitudes, pregnancy, diabetic coma and other conditions of acidosis. On the contrary, the experiments of Van Slyke, Cullen and Stillman (1915) indicate that the buffer value of the blood is increased during protein digestion. This may be due to the passage of $\mathrm{Cl}$ ions from the blood to the stomach with consequent increase in the bicarbonate content of the blood.

It seems probable that the $\mathrm{PH}$ of the blood of all animals is 
near that of human blood since they all contain bicarbonates. The PH of the blood of the conch (Strombus gigas) is about 7.5 , that of the body fluid of the sea urchin (Cassiopea xamachana) 7.7. Although Mines supposes the $\mathrm{PH}$ of the blood of Pecten to be about 6.5 this was determined with indicators and does not seem to hold for the related genus, Pectenella.

\section{Sea Water}

It is maintained by A. B. Macallum (1904) that the salt composition of the blood of mammals is the same as that of the sea during the Cambrian period. In passing from the marine inveretbrates (the osmotic pressure and proportions of whose blood salts is the same as those of the sea) to the mammals, there is a gradual lowering of the osmotic pressure and of the $\mathrm{Mg}$ content of the blood. Macallum supposes that the evolution of the sea took the reverse direction and the osmotic pressure and $\mathrm{Mg}$ content was low in the Cambrian period. The osmotic pressure is constantly increased owing to the accumulation of salts carried in by rivers, which now carry a greater proportion of $\mathrm{Ca}$ and $\mathrm{Mg}$. But the $\mathrm{Ca}$ is constantly being precipitated since the sea is about saturated with calcite and the $\mathrm{Mg}$ alone is relatively increasing. The osmotic pressure of the blood of various animals is given above. The following table gives the relative composition of the ash, $\mathrm{Na}$ being taken as 100 :

$\begin{array}{lcccc}\text { To roo parts Na : } & \mathrm{Ca} & \mathrm{K} & \mathrm{Mig} & \\ \text { Sea water } & 3.94 & 3.57 & \text { II.99 (F. W. IClarke, I9I I) } \\ \text { Medusa (Aurelia) } & 4.13 & 5.18 & \text { Ir.43 (Macallum, rgo4) } \\ \text { Limulus blood } & 3.83 & 5.34 & \text { II.50 (Gotch and Laws, I885) } \\ \text { Sea urchin fluid } & 5.45 & 0.24 & \text { r } 3.27 \text { (Mourson and Schlagdenhauffen, I882) } \\ \text { Sea urchin fluid } & & \text { I2.00 } & 2.20 \text { (Griffiths, I892) } \\ \text { Av'ge of Molluscs } & 5.43 & 12.20 & 3.07 & \text { " } \\ \text { Lobster blood } & 8.03 & 7.12 & 2.88 \text { (Macallum) } \\ \text { Sipunculus (worm) } & 6.55 & \text { I2.70 } & 3.00 \text { (Griffiths) } \\ \text { Dog's serum } & 2.52 & 6.86 & .8 \mathrm{r}\end{array}$

The discrepancies in the proportion of $\mathrm{K}$ ( in sea urchins, for instance) may be due partly to the fact that cells containing a larger proportion of $\mathrm{K}$ were sometimes included, and partly to faulty analysis.

Excised organs and cells of marine animals live for experimental periods in sea water and M. L. Lewis (Ig16) has shown that vertebrate tissues may proliferate in diluted sea water. 
Artificial sea water may be made as follows, out of the purest reagents especially free from $\mathrm{Ba}$, As and heavy metals, dissolved in water that has been redistilled with a silica condensing tube and aerated several days with outside air. The numbers denote cc of normal solutions ( $0.5 \mathrm{~mol}$ in case of bivalent salts) in a liter.

$\begin{array}{lcccccc}\mathrm{KCl} & \mathrm{CaCl}_{2} & \mathrm{MgCl}_{2} & \mathrm{NaCl} & \mathrm{MgSO}_{3} & \mathrm{NaBr} & \mathrm{NaHCO}_{\text {s }} \\ \mathrm{I0.2} & 22 . \mathrm{I} & 50.2 & 48.5 & 57.2 & 0.8 & 2.3\end{array}$

After mixing, the solution is aerated with outside air several hours in order to bring the alkalinity to $\mathrm{PH}=8 . \mathrm{I}-8.2$ which is the $\mathrm{PH}$ of the surface of the ocean. This should show a faint pink with phenolphthalein. The $\mathrm{CO}_{2}$ tension of surface sea water is about 0.04 per cent of an atmosphere, whereas outside air averages 0.03 per cent $\mathrm{CO}_{2}$. It is therefore possible to make the sea water slightly hyperalkaline by prolonged aeration with outside air, but the addition of animals or bacteria quickly brings it back to normal and the problem is to aerate it sufficiently. The curve of $\mathrm{PH}$ with varying $\mathrm{CO}_{2}$ tension is similar to that of blood (Fig. I8) or any other bicarbonate solution.

The other constituents of sea water exist as traces. They may be present as impurities in the salts used in making artificial sea water even in excess of their concentration in natural sea water, or they may get into the water from the air or by solution of the glass vessels used to store it.

In order to investigate the significance of the composition of sea water on marine organisms the effect of changing its composition has often been studied. If the alkalinity is increased to $\mathrm{PH}=9.00$ there is finally a slight precipitate of lime (calcite) and the alkalinity returns to normal, so that it is difficult to increase the alkalinity and keep it so for many days. Herbst ( 1898 ) and Loeb ( $1898 \mathrm{~b}$ ) observed that an increase in the alkalinity increased the rate of development of sea urchin eggs, and $\mathrm{O}$. Warburg ( 1810 a) found that this was accompanied by an increase in respiration of the eggs. Herbst found that $\mathrm{Cl}, \mathrm{Na}$, $\mathrm{K}, \mathrm{Ca}$ and $\mathrm{OH}$ ions are necessary for the development of these eggs from the beginning, but $\mathrm{SO}_{4}, \mathrm{CO}_{3}$ and $\mathrm{Mg}$ are necessary only for later stages. Plants are less affected by changes in the composition of the sea water, but Osterhout (I9I2 b) has found plants that require Na. Diatoms grow better if $4 \mathrm{cc}$ of $\mathrm{n} \mathrm{KNO}_{3}$ 
are added to each liter of sea water, but some algae are not benefitted by it. This high concentration of nitrates is not maintained very long owing to the presence of denitrifying bacteria, which are especially active and abundant in tropical seas.

The constituents of sea water are either nutritive or protective, or both. The $\mathrm{H}_{2} \mathrm{O}, \mathrm{SO}_{4}$ and $\mathrm{CO}_{2}$ are used by green plants in the manufacture of protoplasm, and they require also $\mathrm{PO}_{4}$ and either $\mathrm{NH}_{3}, \mathrm{NO}_{3}$ or $\mathrm{NO}_{2}$, which exist in traces in natural sea water. Traces of $\mathrm{I}, \mathrm{Ca}, \mathrm{Mg}, \mathrm{Fe}, \mathrm{Cu}, \mathrm{Mn}$, Si, etc., may serve a nutritive function to some organisms. The chief constituents of the sea water may, however, be considered protective in that they are not used up. Their presence in definite proportions is necessary in order to preserve life.

The work of Ringer, O. Loew, Loeb and others suggests that these constituents have antagonistic actions, so that a balance is necessary. Osterhout maintains that they either increase or decrease the permeability of cells. These antagonisms of the ions of sea water were demonstrated by the perfused heart of the conch (Strombus) at Tortugas, Fla. The sea water freezes at $-2.03^{\circ}$ and isotonic solutions were made as follows: 0.59 $\mathrm{m} \mathrm{NaCl}, 0.6 \mathrm{~m} \mathrm{KCl}, 0.36 \mathrm{~m} \mathrm{CaCl}_{2}$, and $0.38 \mathrm{~m} \mathrm{MgCl}_{2}$. $\mathrm{Na}, \mathrm{K}$ and $\mathrm{OH}$ ions increase the rate of the heart beat and stop it in systole whereas $\mathrm{Mg}, \mathrm{H}$ and $\mathrm{Ca}$ ions decrease the rate and stop it in diastole. These antagonisms are not perfect, however, although they are slightly more perfect in the action of these ions on the pulsations of the medusa, Cassiopea. The reason for the failure of complete antagonism is probably found in the fact that the organism is made of a number of structures, each pair of antagonistic ions having a certain antagonistic ratio for each structure, and this ratio being different for each structure. In order to obtain the same antagonistic ratio for all structures, a combination of the antagonistic pairs must be made, and the result is sea water. To use Osterhout's hypothesis, $\mathrm{Na}, \mathrm{K}$ and $\mathrm{OH}$ ions increase the permeability and $\mathrm{Mg}, \mathrm{Ca}$ and $\mathrm{H}$ ions decrease the permeability, but it is necessary to have all present in a certain proportion in order that the permeability of all the structures in the organism be normal. It is not sufficient to have a definite ratio between $\mathrm{H}$ and $\mathrm{OH}, \mathrm{Na}$ and $\mathrm{Ca}, \mathrm{K}$ and $\mathrm{Mg}, \mathrm{Na}$ and $\mathrm{Mg}$, or $\mathrm{K}$ and ' $\mathrm{Ca}$. 
A few protective solutions have the following percentage composition:

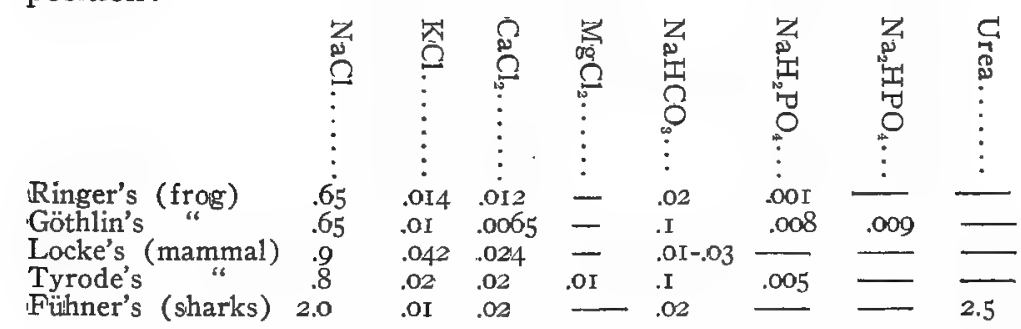

The above solutions may be made nutritive by the addition of .I-.25 per cent glucose.

Many green plants will live in distilled water and need no especially protective solution except that any ion if in very high concentration must be made harmless by the presence of some antagonistic ion. Knop's solution is protective and nutritive for green plants. It is made by dissolving the following numbers of grams in 3-7 liters of water: $4 \mathrm{Ca}\left(\mathrm{NO}_{3}\right)_{2}$, I $\mathrm{KNO}_{3}$, I $\mathrm{KH}_{2} \mathrm{PO}_{4}, 0.5 \mathrm{KCl}$, I $\left(\mathrm{MgSO}_{4} \cdot 7 \mathrm{H}_{2} \mathrm{O}\right)$. It is probable that traces of impurities in this solution are essential since many plants contain additional elements. The analyses of Jost on the ash of a number of land plants show them to contain the following minerals, beginning with the most abundant: $\mathrm{CaHPO}_{4}$, $\mathrm{K}, \mathrm{Mg}, \mathrm{Mn}, \mathrm{Na}_{2} \mathrm{SO}_{4}, \mathrm{Si}, \mathrm{Cl}, \mathrm{Fe}$. The $\mathrm{P}$ and $\mathrm{S}$ entered at least partly into the composition of proteins, but since anions are necessary to combine with the cations, the salts were probably phosphates, sulphates, chlorides and carbonates.

According to Stoklasa (1908) the ash of Azobacter is almost pure $\mathrm{K}_{2} \mathrm{HPO}_{4}$. Ad. Mayer (IgO2) used the following solution for the growth of yeast, expressed as grams dissolved in two liters: I $\mathrm{KH}_{2} \mathrm{PO}_{4}$, o.I $\mathrm{Ca}_{3}\left(\mathrm{PO}_{4}\right)_{2}$, I $\mathrm{MgSO}_{4}$.

Salts of Ais, $\mathrm{Cr}, \mathrm{Zn}, \mathrm{Cd}, \mathrm{Ni}, \mathrm{Co}, \mathrm{Cu}, \mathrm{Hg}, \mathrm{Au}$ and $\mathrm{Pt}$ are said to stimulate the growth and fermentative activity of certain fungi, when in certain minute concentrations. These salts are probably to be considered protective (affecting permeability), since the heavy metal ions are powerful antagonists to some more common cations, and only this antagonistic action explains the necessity of exactly limiting the concentration of the heavy metal.

Bacteriological media are too numerous to be considered here. The PH is very important (see papers of W. M. Clark). 


\section{APPENDIX}

\section{CHEMICAL SUMMARY}

The elements contained in every living cell are as follows:

\begin{tabular}{|c|c|c|c|}
\hline Element & Symbol & Atomic weight & Valence \\
\hline Hydrogen & $\mathrm{H}$ & 1.008 & $\mathbf{I}$ \\
\hline Carbon & C & 12 & 4 \\
\hline Nitrogen & $\mathrm{N}$ & 14.01 & 3 , or 5 , or 4 \\
\hline Oxygen & 0 & I6 & $4(6$ or $12 ?)$ \\
\hline Phosphorus & $P$ & 31.04 & 3 \\
\hline Sulphur & $\mathrm{S}$ & 32.07 & $2,4,6$ \\
\hline Chlorine & $\mathrm{Cl}$ & 35.46 & $I(2,3,4,5 ?)$ \\
\hline Sodium & $\mathrm{Na}$ & 23 & I \\
\hline Potassium & $\mathrm{K}$ & 39.1 & I \\
\hline Magnesium & $\mathrm{Mg}$ & 24.32 & 2 \\
\hline Calcium & $\mathrm{Ca}$ & 40.07 & 2 \\
\hline Iron & $\mathrm{Fe}$ & 55.84 & 3 \\
\hline
\end{tabular}

Other elements sometimes present are as follows:

$\left.\begin{array}{lccc}\quad \text { Element } & \text { Symbol } & \text { Atomic weight } & \text { Valence } \\ \text { Fluorine } & \mathrm{F}^{\prime} & \mathrm{I} 9 & \mathrm{I} \\ \text { Bromine } & \mathrm{Br} & 79.92 & \mathrm{I} \\ \text { Iodine } & \mathrm{I} & \mathrm{I} 26.92 & \mathrm{I}\end{array}\right\}(2,3,4,5$ ?)

None of these elements exist as simple atoms in the cell. The chief compound is water, HOH. It seems probable, from the high surface tension, specific heat and dielectric constant of water, that some molecules have combined (polymerized) to form $(\mathrm{HOH})$. . It is even believed that some of these aggregates are colloidal particles, and cause the blue color of the sea, buit it seems more probable that this blue light is dispersed by water molecules, as the blue sky is produced by dust particles on drops of water in the air. Gases dissolve in water in the form of $\mathrm{O}_{2}$, $\mathrm{N}_{2}$ or $\mathrm{H}_{2}$. Many of the elements exist in solution as salts, formed by the combination of an acid and a base. $\mathrm{Na}, \mathrm{K}, \mathrm{Mg}, \mathrm{Ca}, \mathrm{Al}, \mathrm{Mn}$ and $\mathrm{Fe}$ combine with $\mathrm{O}$, forming oxides, which combine with water forming hydroxides, which are bases. $\mathrm{F}, \mathrm{Cl}, \mathrm{Br}$ and $\mathrm{I}$ combine with $\mathrm{H}$ to form acids and $\mathrm{Si}, \mathrm{P}, \mathrm{S}, \mathrm{C}, \mathrm{N}$ and $\mathrm{O}$ may enter into the composition of acids.

The compounds of carbon are so complex and numerous as to form a special group, organic compounds. This is subdivided into the open chain or aliphatic series and the ring compounds or aromatic series.

The paraffins are compounds of carbon and hydrogen in open chain. Starting with methane, $\mathrm{CH}_{3}$, a combination of two molecules gives ethane, $\mathrm{C}_{3} \mathrm{H}_{8}$, because two hydrogen atoms are eliminated and the valences thus 
freed are used to connect the two carbon atoms. In a similar way propane, $\mathrm{C}_{3} \mathrm{H}_{8}$, and butane, $\mathrm{C}_{4} \mathrm{H}_{10}$, and an indefinite number of higher compounds are formed.

In the paraffins all of the valences of the carbon atoms are filled and no more hydrogen atoms can be attached to the molecule without splitting it between two carbon atoms, and therefore these compounds are said to be saturated. In the unsaturated hydrocarbons, however, more hydrogen atoms may be attached without splitting the molecule, which is thereby converted into a saturated compound. There are no free valences in the unsaturated compounds but two carbon atoms are united by more than one bond, and by opening up one bond two free valences are formed for the attachment of two hydrogen atoms. Thus in ethylene, $\mathrm{C}_{2} \mathrm{H}_{4}$, there is a double bond between the carbon atoms and acetylene, $\mathrm{C}_{2} \mathrm{H}_{2}$, there is a triple bond between the two carbon atoms.

The first products of the oxidation of the paraffines are the alcohols, methyl alcohol, $\mathrm{CH}_{8} \mathrm{OH}$, ethyl alcohol, $\mathrm{C}_{2} \mathrm{H}_{8} \mathrm{OH}$, propyl alcohol, $\mathrm{C}_{3} \mathrm{H}_{7} \mathrm{OH}$, and so on. Alcohols may combine with bases, but where water is formed by the combination the compound may decompose again. If the presence of water be eliminated the compound is relatively stable. Thus when metallic sodium is dropped into ethyl alcohol, sodium ethylate is formed with the evolution of hydrogen.

$\mathrm{C}_{2} \mathrm{H}_{5} \mathrm{OH}+\mathrm{Na}=\mathrm{C}_{2} \mathrm{H}_{5} \mathrm{ONa}+\mathrm{H}$ and $2 \mathrm{H}=\mathrm{H}_{2}$.

The combination of an alcohol with an acid forms an ester, thus:

$$
\mathrm{C}_{2} \mathrm{H}_{5} \mathrm{OH}+\mathrm{H}_{2} \mathrm{SO}_{4}=\mathrm{C}_{2} \mathrm{H}_{6} \mathrm{HSO}_{4}+\mathrm{H}_{2} \mathrm{O} \text {. }
$$

If an ester is warmed with a strong base, the base combines with the acid and the alcohol is set free, a process known as saponification, thus:

$$
\mathrm{C}_{2} \mathrm{H}_{5} \mathrm{HSO}_{4}+\mathrm{Ca}(\mathrm{OH})_{2}=\mathrm{C}_{2} \mathrm{H}_{5} \mathrm{OH}+\mathrm{CaSO}_{4}+\mathrm{H}_{2} \mathrm{O} \text {. }
$$

The alcohols with one $\mathrm{OH}$ group, like the ones given above, are called monatomic. The highest monatomic alcohol found in cells is cholesterol, $\mathrm{C}_{27} \mathrm{H}_{46} \mathrm{OH}$, a waxy substance, insoluble in water and crystallizing in plates.

Alcohols with three OH groups are called triatomic. The one found in cells is glycerol, $\mathrm{C}_{3} \mathrm{H}_{5}(\mathrm{OH})_{3}$, which is soluble in water, and its esters with fatty acids are called fats.

By oxidation of alcohols we obtain aldehydes. Methyl alcohol gives form-aldehyde, thus: $2 \mathrm{CH}_{3} \mathrm{OH}+\mathrm{O}_{2}=2 \mathrm{H} \cdot \mathrm{CHO}+\mathrm{H}_{2} \mathrm{O}$. In the aldehyde group, $\mathrm{CHO}$, the oxygen is united with the carbon by a double bond, which makes the aldehyde capable of further oxidation, in fact it has such an affinity for oxygen as to be a mild reducing agent. The product of such an oxidation of an aldehyde is a fatty acid and the aldehyde is named for the acid that it forms and not for the alcohol from which it is formed, where there is a different name for the alcohol and acid. Thus we have methyl alcohol, formaldehyde and formic acid and we have ethyl alcohol, acetaldehyde and acetic acid, but propyl alcohol, propylaldehyde and propionic acid.

The CHO group of the aldehyde contains the terminal carbon atom of the chain. If an intermediate carbon atom of the chain bears the double bond we have a $\triangle \mathrm{CO}$ group, and the substance is called a ketone. Thus, $\mathrm{CH}_{3} \mathrm{CH}_{2}{ }^{\prime} \mathrm{CHO}$ is propyl aldehyde, but $\mathrm{CH}_{8} \mathrm{COCH}_{3}$ is dimethyl ketone (acetone).

The carbohydrates are aldehydes or ketones of polyatomic alcohols, in which the hydrogen and oxygen are usually in the proportion to form water. Aldehydes have a tendency to polymerize or combine to form langer molecules. Formaldehyde in alkaline solution polymerizes to form a series of sugars with six carbon atoms. This seems also to be the mode of formation of sugar in the green plant. The carbonic acid, $\mathrm{H}_{2}{ }_{2} \mathrm{CO}_{3}$, is reduced by the action of sunlight and the fluorescent chlorophyll to $\mathrm{HCHO}$, the oxygen removed from the carbonic acid combines with 
water to form hydrogen peroxide, which is immediately decomposed by the catalase of the cell with the liberation of $\mathrm{O}_{2}$. The formaldehyde would destroy the cell if it were not immediately polymerized by the action of the green bodies, chloroplasts.

The most important sugars are the hexoses or those with six carbon atoms, which are asymmetrical. The carbon atom has four valences and if a different kind of atom or group is attached to each of these valences, the molecule is asymmetrical, that is to say, no point, line or plane of symmetry exists. It is then possible to arrange the groups around the caribon atom in two different ways so that one form of molecule will be the mirrored image of the other. Such asymmetry can be detected because a molecule containing one asymmetric carbon atom rotates the plane of polarized light to the right or the left. If a molecule contains two asymmetric carbon atoms, both atoms may rotate in the same direction or in opposite directions, and if the rotation is equal in opposite directions the molecule appears inaotive to polarized light. There are often obtained mixtures of equal numbers of dextro-rotatory and laevo-rotatory molecules, the mixture does not affect polarized light and is called racemic.

The hexoses that are readily assimilable by mammalian cells are:
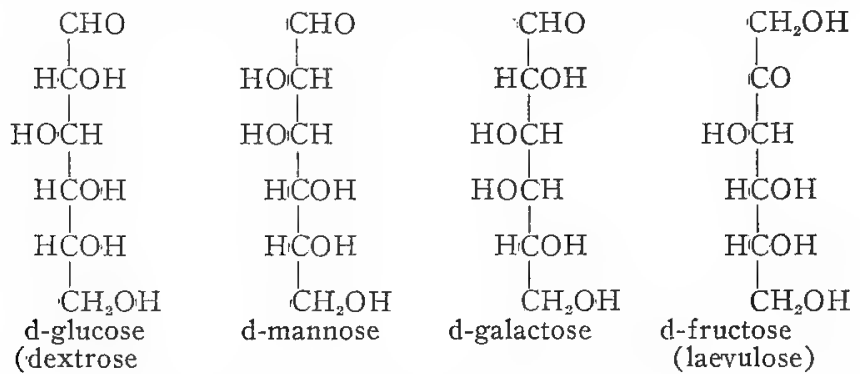

This is the nomenclature of Fischer, based on structural formulae. All of these sugars are aldehydes (aldoses) and rotate polarized light to the right, except d-fructose (a ketose) which is usually icalled laevulose because it rotates polarized light to the left.

On the oxidation of I g. glucose abrout 3.74 calories of heat are produced.

Glucose, fructose and galactose, when reduced, are converted into the alcohols, sorbite, mannite and dulcite. Glucose on mild oxidation, yields gluconic acid, with the $\mathrm{COOH}$ group formed from the $\mathrm{CHO}$ group. On further oxidation another $\mathrm{COOH}$ group is formed on the other end of the chain, and thus the dibasic acid, saccharic acid is formed. Glucose, as well as other hexoses, is easily transformed into lactic acid without oxidation. One molecule of glucose yields two molecules of lactic acid, $\mathrm{CH}_{3} \mathrm{CHOHCOOH}$, when acted on by lime in sunlight or by bacteria, or other living cells.

As aldehydes or ketones, the hexoses are reducing substances, but this property may be lessened spontaneously, in solution, by what is called lactone formation. In this, the double bond between one carbon and oxygen atom, to which the reducing character is due, opens out and one bond becomes applied to a distant carbon atom in exchange for an $\mathrm{OH}$ group.

Glucose may combine with other substances, forming glucosides. This combination is usually preceded by lactone formation. Two hexose molecules may combine by the opening out of a double bond and the elimination of one molecule of water, forming a disaccharide molecule. Thus 
glucose and glucose form maltose, glucose and galactose form lactose, and glucose and fructose form saccharose or cane sugar. One gram of a 'disaccharide on oxidation produces 3.95 calories of heat.

Pentoses, with five carbon atoms, are found in many plant cells.

Polysaccharides are formed by the combination of simple sugar molecules. Gums, pectens and mucilages are formed of monosaccharides, and starch, dextrin, glycogen and cellulose are formed of glucose. The molecular weight of starch is estimated at between 1260 and 32400 . Its osmotic pressure is so small that it appears to be zero.

By the oxidation of the $\mathrm{CHO}$ group of the aldehydes the $\mathrm{OOOH}$ group of the organic acids is obtained. The open chain acids are called fatty acids and bear the same name as the aldehydes from which they are formed. In the series, formic, acetic, propionic, butyric, valerianic and caproic, each member has one more carbon atom than the one preceding. The fatty acids most common in animal cells have even numbers of $\mathrm{C}$ atoms. These acids are butyric, caproic, caprylic, capric, lauric, myristic, palmitic and stearic. The solubilities of these acids in water decrease as the number of carbon atoms increases, the higher members of the series ibeing insoluble.

In the same way that unsaturated hydrocarbons differ from paraffines in the presence of dotrble or treble bonds between adjacent carbon atoms, so the unsaturated fatty acids differ from those given above. The unsaturated fatty acids, oleic, linoleic have respectively I and 2 double bonds. Linolenic has 3 double bonds. These unsaturated acids may take up oxygen and become saturated, and in this way they act as reducing substances.

The oxy-acids contain more oxygen than the normal acids. Propionic acid is $\mathrm{CH}_{8} \mathrm{CH}_{2} \mathrm{COOH}$ whereas oxypropionic, usually called lactic acid, is $\mathrm{CH}_{8} \mathrm{CHOH}$ iCOOH.

Keto acids contain the ketone group, and also the $\mathrm{COOH}$ group.

Fatty acids combine with bases to form salts, which in case of the higher members of the series are called soaps. The soaps formed by action of alkaline earths on the higher fatty acids a1e insoluble in water whereas the lower fatty acids form soluble soaps with these bases. Alkali soaps hydrolize in water, forming a fine emulsion of fatty acid that makes the solution opalescent.

Fatty acids combine with ammonia, but the compound may be of either of two forms. With ammonium hydrate, which is a true base, they form ammoniacal soaps, but with dry ammonia and a dehydrating agent they form acid amides containing one less molecule of water. In ammoniacal soaps $\mathrm{N}$ is pentivalent in the group $\mathrm{COONH}_{4}$, whereas in amides nitrogen is trivalent in the group $\mathrm{CON}_{2}$.

The esters of fatty acids with alcohols are called fats. The triatomic alcohol glycerol is the most common but the monatomic alcohol cholesterol and other alcohols are also found.

Since glycerol is a triatomic alcohol, it can combine with one, two or three fatty acid molecules, forming mono-, di-, and triglycerides, of which the last is the most common. Fat globules found in cells are usually mixtures of triglycerides and fatty acids. In the analysis of fats, the acid number denotes the content of free fatty acids, the saponification number, or combining power with hot $\mathrm{KOH}$, is an index of the average of molecular weight of each fatty acid divided by its valence, and the iodine number is an index of the content in unsaturated fatty acids. The percentage of volatile fatty acids is usually determined, but the separation of all of the fatty acids involves much labor.

The oxidation of I g. fat may yield as much as 9.5 calories of heat.

Mono or diglycerides may combine with other stubstances by means of the remaining alcohol groups. Such compounds are included under the loose term, lipoids, but have been called by Leathes phospholipines, 
galactolipines and lipines. Phospholipines are diglycerides in which the third alcohol group is combined with phosphoric acid. Since phosphoric acid is trivalent it can combine with two other substances. In lecithin it combines with only one other substance, the organic base, choline. The phospholipines are unstable and are partly decomposed and oxidized by extraction with hot solvents in the presence of air, and hence their composition has not been entirely settled in all cases.

In galactolipines, galactose takes the place of glycerol. Lipines contain nitrogen but no carbohydrate or phosphoric acid. From fatty acids and ammonia may be prepared not only acid amides, but also amino acids (lactic acid with $\mathrm{NH}_{8}$ and a dehydrating agent forms alanine). In the mono-amino acids obtained from mammalian cells the $\mathrm{NH}_{2}$ group is attached to the carbon atom next to the $\mathrm{COOH}$ group. These acids are called alpha amino acids. In the diamino acids, an additional $\mathrm{NH}_{2}$ group is attached to some other carbon atom of the chiain.

The amino acids have both acid and basic properties and are therefore called amphoteric. In the diamino acids the basic property predominates. Amino acids may be derived from dibasic acids, containing two COOH groups and in this case the acid property markedly predominates.

The proteins are compounds of amino acids in which the $\mathrm{NH}_{2}$ group of one molecule has reacted with the $\mathrm{COOH}$ group of another. The complete structure of none of the proteins is known, but many amino acids have been separated from the decomposition products, among which are: The monoamino acids, amino acetic or glycine, aminopropionic or alanine, amino isovalerianic or valine, and aminoisocaproic or leucine. The dibasic amino acids are aspartic and glutamic acids. The diamino acids are lysine, arginine, and diaminotrioxydodecoic acid. Cystine contains two atoms of sulphur. Other amino acids contain cyclic compounds, which will now be considered.

Whereas in the compounds thus fat considered the carbon atoms are arranged in an open chain, in the cyclic compounds they are in the form of a closed ring. The most important of these rings is the benzol ring containing 6 carbon atoms. Although this ring has only $6 \mathrm{H}$ atoms there seem to be no double bonds between the carbon atoms, or if there are double bonds they do not behave as the double bonds of the unsaturated open chain compounds. In benzol, ${ }^{\circ} \mathrm{C}_{6} \mathrm{H}_{6}$, each $\mathrm{C}$ atom is united to one hydrogen atom. The substitution of one or more hydrogen atoms by other atoms or groups gives rise to a host of compounds. When only one hydrogen is substituted it is immaterial which carbon atom it is attached to, but by its presence the other carbon atoms lying at different distances from the first one are designated as ortho, meta and para, and groups that may subsequently be attached to them are in the ortho, meta, or para position.

Of the mono-su'bstiturtion products of benzol we have aniline, $\mathrm{C}_{8} \mathrm{H}_{5} \mathrm{NH}_{2}$, benzol-sulphonic acid, $\mathrm{C}_{8} \mathrm{H}_{5} \mathrm{SO}_{3} \mathrm{H}$, phenol, $\mathrm{C}_{6} \mathrm{H}_{5} \mathrm{OH}$, toluol, $\mathrm{C}_{8} \mathrm{H}_{5} \mathrm{CH}_{3}$, benzyl alcohol, $\mathrm{C}_{6} \mathrm{H}_{5} \mathrm{CH}_{2} \mathrm{OH}$, benzaldehyde, $\mathrm{C}_{8} \mathrm{H}_{5} \mathrm{CHO}$, and benzoic acid, $\mathrm{C}_{6} \mathrm{H}_{5} \mathrm{COOH}$.

Of the di-substitution products we have catechol (ortho-hydroxyphenol), resorcinol (meta-hydroxyphenol), hydroquinone (para-hydroxyphenol), salicylic acid (ortho-hydroxy benzoic aoid), and tyrosin (para-hydroxyphenylalanine). Tyrosin is an amino acid found in proteins.

Some tri-substitution products are pyrogallol, homogentisic acid, picric acid and adrenaline. Adrenaline is secreted by cells of the adrenal medulla and is a specific stimulant (or in some cases inhibitor) of smooth muscle cells, although its action is supposed not to be on the whole cell surface but on a theoretical receptor substance.

The pyrrol ring contains I nitrogen and 4 carbon atoms and is contained in proline and oxyproline, amino acids found in proteins. Tryptophane, another amino acid found in proteins, contains a combination of 
benzol and pyrrol rings and may be transformed into indol and skatol by the action of bacteria.

The imidazol ring contains three carbon and two nitrogen atoms

$$
\underset{\mathrm{H}-\mathrm{C}-\sum_{\mathrm{N} / /}^{\mathrm{C}} \mathrm{H}}{\mathrm{H}}=\stackrel{\mathrm{H}}{\mathrm{N}}=\text { Imidazol ring. }
$$

and is contained in histidine, an amino acid found in proteins.

When protein is split into amino acids by any method, not all of the protein is thus accounted for.

Now that the building stones of the proteins have been discussed, it is well to consider a few characteristics of these interesting substances.

Proteins consist of the same amino acids but in different proportion. Glycine is absent from some proteins. Tyrosine and tryptophane are absent from gelatine.

Proteins consisting almost entirely of amino acid's are called simple proteins to distinguish them from those combined with other substances. In oxyhemoglobin, a simple protein is combined with hematin, an iron containing organic compound loosely combined with one molecule of $\mathrm{O}_{2}$.

The nucleins that have been isolated from the nuclei of some cells are composed of a simple protein and nucleic acid. The nuclein may combine with more protein to form nucleo-protein. Nucleic acid contains phosphoric acid and one or more purine bases, adenine or guanine, allied to uric acid.

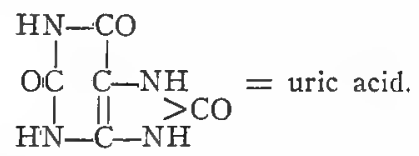

The glycoproteins, such as the mucins, contain a high percent of carbohydrate groups, which are also found in tucleic acid.

The phosphoproteins, vitellin, casein, contain phosphoric acid that is not in the form of nucleic acid or lecithin.

The lecitho-proteins are hypothetical, although both lecithin and protein exist in the crystalline yolk bodies of the frog's egg. Hoppe Seyler considered such substances to be true chemical compounds but others have regarded them as mixtures. One argument against their being mixtures is that the yolk bodies are clear and not turbid as colloidal gels or sols containing lecithin are.

The molecular weight of simple proteins has been estimated as varying from 14,000 to 30,000 , whereas that of oxyhemoglobin is about 16,000 .

Proteins have both acid and basic characters since they contain both $\mathrm{COOH}$ and $\mathrm{NH}_{2}$ groups. Their acid character predominates when in alkaline solution and basic character predominates when in acid solution. Proteins are precipitated by heavy metals and the color bases of many dyes and by the same aoids that precipitate alkaloids, namely tannic, metaphosphoric, picric, phosphomolybdic, phosphotungstic, tri-iodo-hydriodic, chromic, bichromic, and the color acids of many dyes.

Certain bacteria have the power of splitting off ammonia from the amino acids of proteins, a process known as deamination. If the deamination is accompanied by hydrolysis oxyacids are formed, whereas if reduction takes place fatty acids are formed. In the eggs of certain fish fats are formed from proteins. The glycerine necessary in the process anises from proteins and may possibly arise in part in the following manner. In the conversion of glucose into lactic acid glyceric aldehyde is formed, which by reduction becomes glycerol. 


\section{ABBREVIATIONS USED IN LITERATURE LIST}

AA Anatomischer Anzeiger.

AAP

AB

A'Bit

$A n B$

$A C$

AiCJ

$A C P$

$\mathrm{AE}$

AEP

AF

AGP

AH

AIB

AIM

A'IP

AIPd

A'TP'T

AJ.A

A.JB

Arch. f. Anat. Physiol. Engelmann).

Archiv de Biologie (Van Beneden).

Archiv für Botanik.

Annals of Botany.

Ann. IChem. u. Pharm. (Liebig) (= An. Pm.)

A.merican Chemical Journal.

Ann. d. Chim. et Phys. (= A. Phys.).

Arch. Entwicklungsmech. (Roux).

Arch. f. Exp. Path. u. Pharmakologie.

Archivio di Fisiologia.

Arch. gesammt. Physiol. (Pflüger).

Archiv für Hygiene.

Archives Ital. d. Biol.

Archives of Internal Medicine.

Arch. Tnternat. de Physiol.

Arch. Internat. d. Pharmacodynam.

Ann. d. l'Ins. d. Pasteur.

American Journal of Anatomy.

AJDIC

American Journal of Botany.

AJMS

AJP

AJS

AK

A'K:G

A.MBG

A.N

ANd

ANY'A

$\mathrm{APk}$

$\mathrm{APm}$

AR

Am. J1. of Diseases of Children.

Am. J1. of the Medical Sciences.

Am. J1. of Physiology.

American Journal of Science.

A rchiv für Kinderheilkunde.

Arb. a. d. kais. Gesundheitsamt.

Annals Missouri Bot. Garden.

American Naturalist.

Arch. Neerland.

Ann. N. Y. Acad. of Science.

Ann. đ. Physik (Poggendorf, Wied., Drud.) (= J1. Pk., N. J. Pk., A. Pk. C.)

ASB

ASNZ

ASPN

AUG

$A Z$

AZEG

BiARB

BASC

$\mathrm{BB}$

$\mathrm{BBF}$

BBS

$\mathrm{BC}$

$\mathrm{BCB}$

BCPP Beiträge Chem. Physiol. u. Pathol. (Hofmeister).

Archiv für Pharm.

Anatomical Record.

Arch. d. Sci. Biol.

Ann. d. Sci. Natur. et Zool.

Aroh. Soc. Phys. Nat. Geneva.

Ann. d. I'Univer. de Grenoble.

Archiv für Zellforschung.

Arch. d. Zoolog. Experim. et Gener.

Bull. d. 1'Acad. Roy. 'Belgique.

Bull. Acad. Sc. Cracow. Math. Nat.

Biological Bulletin.

Bull. U. S. Bureau of Fisheries.

Bull. U. S. Bureas of Standards.

Biologisch. ICentralblatt.

Biochemical Bulletin. 


\begin{tabular}{|c|c|}
\hline 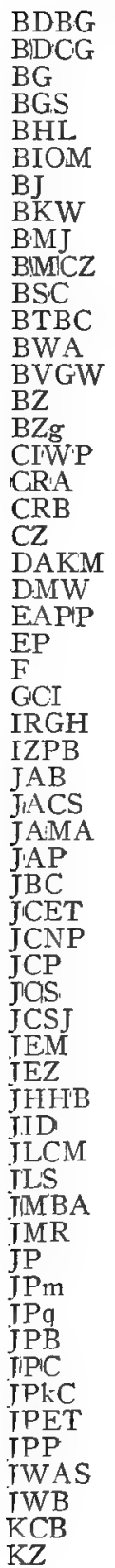 & 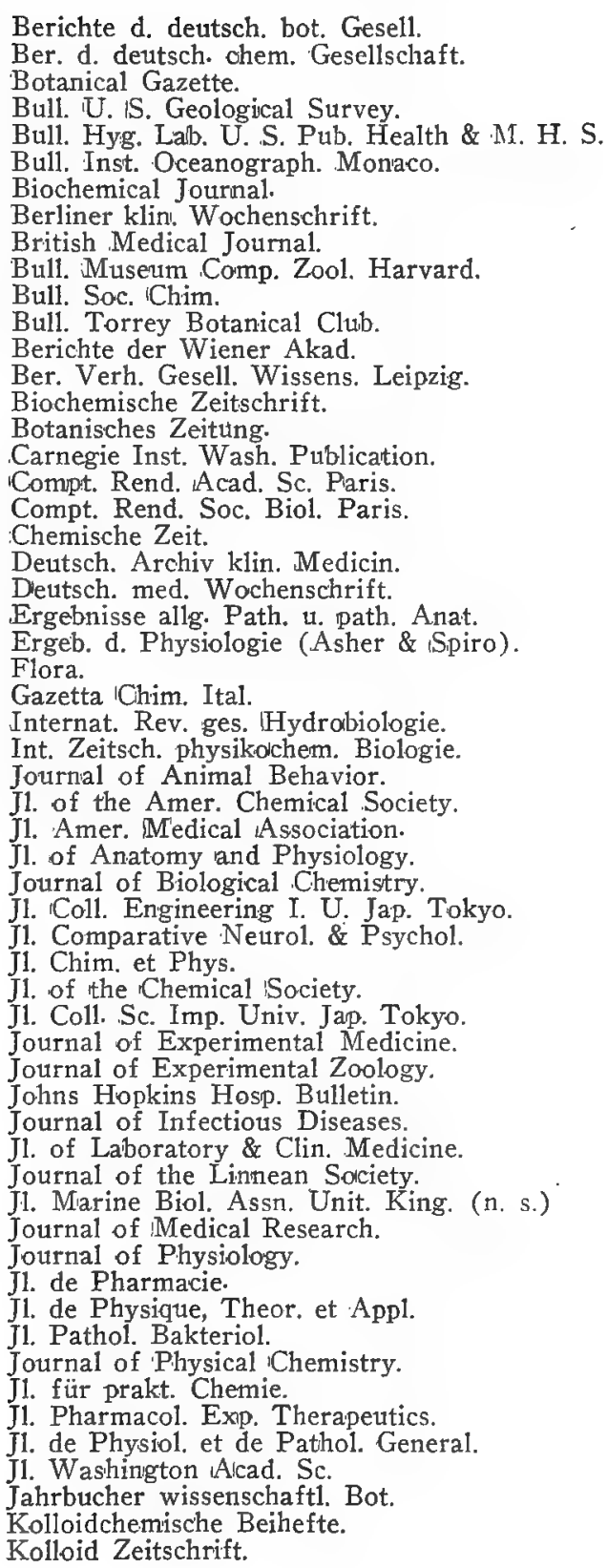 \\
\hline
\end{tabular}




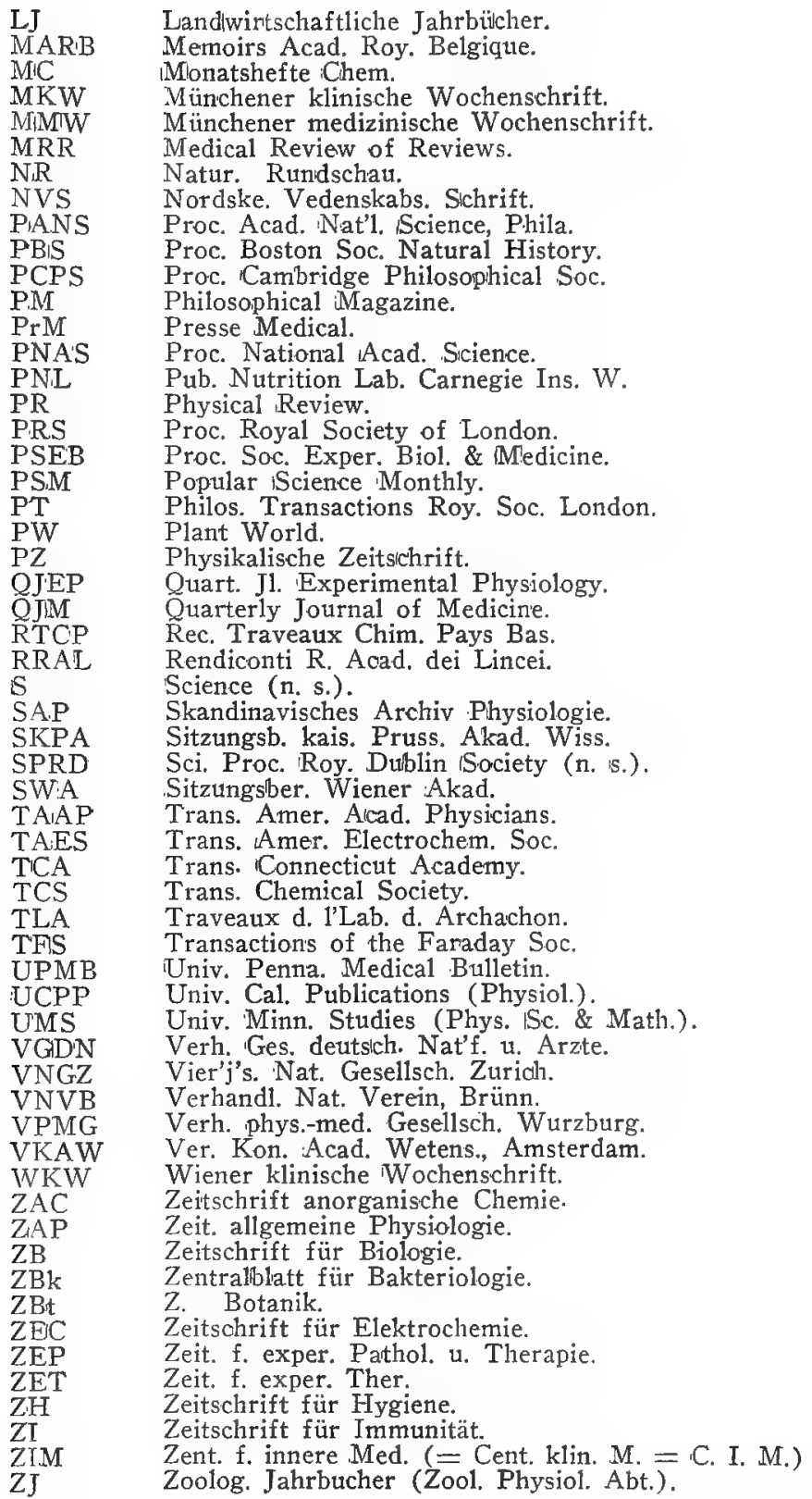




\section{ABBREVIATIONS}

ZK Zeitschrift für Kinderheilkunde.

ZKMM

$Z N$ Zeitschrift für klinisch. Medizin.

$Z P$

$\mathrm{ZPa}$

ZPk

ZPC

ZPkC

ZRM

Zeit. für Nervenheilkunde (Deutsche).

Zentralblatt für 'Physiologie.

Zeitschrift für Pathologie.

Zentralblat für Physik.

ZWM

Zeitschr. für physiolog. Chemie.

Zeits. für Physikalische Chemie.

Zeitschrift für Rationell. Medizin.

ZWZ

Zeit. für wissenschaft. Mikroskopie.

Zeit. für wissenschaftlich. Zool. 


\section{LITERATURE LIST}

Abegg, Auerbach \& Luther I9'I "Messungen Elektromot. Krafte gal. Kette." Halle.

Abderhalden I898 Analysis of mammal blood. ZPC xxv 65.

Igog Milk-droplet membranes. ZPC lix I3.

I910 "Handbuch d. Biochem Arbeitsmethoden." Berlin.

I9I I "Biochem. Handlexikon." Berlin.

— \& Gigon I907 Yeast (splitting dipeptids) inhibited by 1-amino acids. ZPC lii $25 \mathrm{I}$.

\& Guggenheim 1008 Shaking enzymes. Asymmetry. ZPC liv 331.

\& Hall roo8 "Text Book of Physiol. "Chem." N. Y.

\& Pringsheim I909 Asymmetry and enzyme action. ZPC lix 249.

Abel, Rowntree \& Turner I9I4 Diffusible constituents of blood. JPET $\checkmark 275$.

Abl 1907 Anesthetic and electrode potential. Dissertation, Bonn.

Acree \& Johnson I907 Enzymes displace equilib. pt. ACJ xxxviii 258.

Adrian I9I4 All-or-none law. JP xlvii 460.

Agazotti Igo6 PH of blood at low atm. pressure. RRAL (5) xv 48I.

Albutt I9ro Blood viscosity. QJM iv 342 .

Alcock \& Lynch I9II $\mathrm{K}$ in nerve, JP xliii I07.

Alexander \& Cserna I9I3 Narcosis and brain respiration. BZ liii I0o.

Allee I9I4 Rheotaxis and KCN. JEZ xvi 397.

— \& Tashiro I9I4 Rheotaxis and $\mathrm{CO}_{2}$ production. JAB iv 202.

Allemand $19 \mathrm{I} 2 \mathrm{PH}$ and rennin action. $\mathrm{BZ} \times 1 \mathrm{v} 346$.

Allen, F. I9I6 Diabetes. JAMA lxvi r.525.

Allen, G. IgI5a Bile and surface tension of urine. JBC xxii 503. I9r5b Reversibility of rheotaxis. BB xxix III.

Allen, E. \& Nelson I9ro Diatom culture. JMBA viii $42 \mathrm{I}$.

Allen, H. I9I3 "Photoelectricity: the Lib. of Electrons by Light." London.

Armstrong, E. I903 Enzymatic splitting and synthesis of sugars. J.CS Ixxxiii I305.

I904 Action of invertase inhibited by fructose. PRS (B) 1xxiii 500, $516,526$.

Armstrong, H. I908 Properties of water. PRS (A) 1xxxi 80.

Arrhenius I887 Ionization. $\mathrm{ZPkC}$ i 631 .

1889 Osmotic pressure and vapor pressure. ZPkC iii II5.

1892 Usually ions decrease and non-electrolytes increase viscosity of water. $\mathrm{ZPkC} \times 5 \mathrm{I}$. I90I "Lehrbuch der Elektrochemie."

Asher \& Karaulow I9Io Permeability of salivary cells to sugar. BZ xxv 36 . \& Spiro I902-I9I.5 Ergebnisse der Physiologie. Wiesbaden.

\& Waldstein I906 Increased blood pressure not diuretic. BZ ii I.

Atkins IgIoa $\Delta$ Marine vertebrates. BJ v 2 I3.

r9Iob Beans not semipermeable. SPRDS xii 35.

I9I0c $\Delta$ Plant juices. SPRDS xii 463.

Aubert rgr2 Thermo-osmose. ACP xxvi I45, 55 I.

Auer \& Meltzer I916 $\mathrm{MgSO}_{4}$ anesthesia. JPET xxiii $64 \mathrm{I}$.

Auerbach 19r2 EH of $\mathrm{N} /$ io Cal. Elec. $=.337,0^{\circ}-25^{\circ}$. ZEC xviii r 3. I912 $\mathrm{PH}$ of $\mathrm{N} /$ Io $\mathrm{NaHCO}_{8}=8.3$ at $25^{\circ}$. AKG xxxviii 242 . I9I3 PH for Trypsin on Peptone $=8.3=\mathrm{NaHCO}_{3}$. BZ xlviii 425 . 
Austrian I9I ' Case of visc, blood $=$ 13.6. JHHB xxii 9.

Bach \& Chodat I904 Oxygenase and peroxidase. BDCG xxxvi 606.

Bachman I9I2 $\Delta$ of amphibian eggs. AGP cxlviii I4I.

- \& Runnstrom Ig09 $\Delta$ of frog's eggs $=.045$. BZ ccii 290 .

I9I2 Unfertilized frog's egg swells more than fertilized. AGP cxliv 287.

\& Sundberg I9I2 $\Delta$ of tadpoles. AGP cxlvi 2I2, 287.

Bachmann IgI2 Ultramicroscopy of soaps. KZ xi I45.

Bachmetjew I900 Undercooling of insects. ZWZ 1xvii 529.

Bäyer $1902 \mathrm{O}_{2}$-Need of nerve. ZAP ii 169 .

Bainbridge I902 Bile salts increase secretion and lymph production of liver. JP xxviii 204.

- Collins \& Menzies I9I3 Frogs' glomeruli secrete isotonic urine. PRS (B) Ixxxvi 353.

Baltzer IgI I Mitosis. AE xxxii 500.

Bancels, des 1909 Effect of ions on Polarization. CRA cxlix 316.

Bancroft 1906 Reversal of galvanotropism. UCPP iii 2I, JP xxiv 444.

Banta I9I2 Development and respiration. PSEB ix I04.

Barcroft I9I4 "The Respiratory Function of the Blood." Cambridge University Press.

\& Brodie I905 Osmotic work and kidney metabolism. JP xxxiii 52.

\& Camis 1909 Dissociation of oxyhemoglobin. JP xxxix II8.

\& Hill I9I0 Effect of temperature on dissociation of oxyhemoglobin. JP xxxix 81.

\& Straub 19Io Kidney metabolism and osmotic work. JP xli I45.

Barker, Hirschfelder \& Bond r9to Electrocardiogram. JAMA Iv I350.

Banta \& Gortner I9I5 Displacement of organ forming substance. JEZ xviii 433.

Barger I904 Microtensimeter. TCS 1xxxv 286.

\& Starling I9I5 Adsorption of iodine. TCS cvii 4II.

Barlow 1896 Infusion of sugar causes greater hydremia than urea. JP xix 418.

Bartell Igr I Permeability. JPC xv 659.

1912 Diameter of pores and osmose. JPC xvi 318.

I9I4 Negative osmose. JACS xxxvi 646 .

- \& Hocker I916 Negative osmose and diffusion emf. JACS xxxviii 1029, 1036.

Bartelzko I909 Freezing and plasmolysis. JWB xlvii 57.

Barns I889 Suspensoids precipitated by ions not by electrolytes. AJS xxxvii I22.

Bataillon 19I0 Parthenogenesis with foreign sperm. AZEG (5) vi Ior. I9II Parthenogenesis by pricking and electricity. CRB 1xx 562.

1912 Parthenogenesis. ASNZ (9) xvi 249.

Batelli \& Stern Ig07 Cell respiration. JP ix I, 34, 228, 4I0, 737.

I909 Oxidation. BZ xxi 487 , xxxiv 263.

19 I0 Oxidation. BZ xxiii 145 .

I9I3 Anesthetics precipitate protein. BZ lii 226, 253.

Bates I9I2 Faraday $=96500$ coulombs. Thesis, U. of Ill.

Bauer I9I3 Model of electric organ. ZEC xix 590.

Bayliss 1906 Adsorption and electric charge. BJ $\mathrm{i} 173$.

1908 Frog's skin, or bag of Congo red solution acts as electric rectifyer. BZ xi 226.

1909 Osmotic pressure of Congo red. PRS (B) $1 \times x x i 269$.

I9I0 Membrane hydrolysis. KZ vi 23.

I9II Permeability and polarization. PRS (B) Ixxxiv 245.

I9I3 Asymmetry and enzyne action. JP xlvi 236.

I9r5a "Principles of General Physiology." Longmans.

1915 b Enzymes may act in non-solvent media. JP 1 85, S xlii 509.

1916 Viscosity and blood pressure. JP 1 (p. xxiii). 
Beard \& Cramer I9I5 Surface tension and ferments. PRS (B) Ixxviii 575. Beccari r9r5 K within physiol. limit stimulating to muscle. AIB 1xiii 293. Bechhold I904a "Irregular Series" of ionic and colloidal precipitation, Bacteria are anodic. $\mathrm{ZPkC}$ xlviii 385 .

I904b Electrolytic solution tension and precipitating power. ZPkC xlviii 406.

1907 Permeability of membranes. ZPkC $1 x 257$.

Igo8 Ultrafilter. ZPkC lxiv 328.

I9I2 "Die Kolloide in Biologie und Medizin." Dresden.

\& Ziegler 1906 Diffusion in gels. ZPkC lvi 105.

IgIo Solubility of uric acid in serum. BZ xxiv 146.

Beddard 1902 Dyes not excreted by glomeruli of frog's kidney, but by tubules. JP xxviii 20.

Begun, Hermann \& Münzer I9I5 $\mathrm{Alv} \mathrm{CO}_{2}$ (Plesch) 6\%. Drank $20 \mathrm{~g} \mathrm{HCl}$ and Alv. $\mathrm{CO}_{2}$ fell $\mathrm{I} \%$. BZ $1 \times x \mathrm{xi} 255$.

Bein 1899 Effect of membrane on transport numbers. ZPkC xxviii 439.

Benecke I $898 \mathrm{Ca}$-free water causes cells to fall apart. JWB xxxii 474.

Benedict \& Cathcart I9r3 Muscular work. PNL (No. I87).

Benedikt Ig06 PH of diabetic blood. AGP cxv I06.

Beniasch I9I2 Acid agglutination. ZI xii 268.

Bennett \& Cole r9I2 Regeneration of storage cells. TAES.

Benson 1902 Adsorption on foam. JPC vii 532.

Benson \& Wells I9Io Antolysis. JBC viii 6I.

Benrath \& Sachs I905 Formation of $\mathrm{HCl}$ by stomach. AGP cix 460.

Bequerel Igor Seeds survive complete dessication. CRA cxxxix I72I.

Berczeller I9I3 Change of surface tension of colloids. BZ liii 232.

I9I4 Surface tension of alkaline solutions of lecithin increased by ether or chloroform. BZ Ixvi 226.

\& Csaki I9I3 Surface tension of alkaloids. BZ liii 238.

Berg IgI2 Muscle contraction. BCB ii IOI.

Bergeim Igr4 Origin of gastric $\mathrm{HCl}$. PSEB xii $2 \mathrm{I}$.

Berkeley \& Hartley Igo4 Osmotic pressure of sugar. PRS lxxiii 436. 1906 Osmotic pressure of conc. sol. PT (A) ccvi $48 \mathrm{I}$.

Bernoulli I9I3 Sulphates and nitrates temporarily reduce effect of bromide intoxication. AEP 1xxiii 353.

Bernstein, E. \& Simons I9I I Meiostagmine reaction. AJMS cxlii 852.

Bernstein, J. I866 Polarizability of nerve lessened by stimulants. AP 596. I900 Imitation of ameba. AGP 1 xxx 628.

IgOI Surface tension theory of muscle contraction. AGP 1xxxv 27 I. I902 Theory of bioelectric currents. AGP xcii 52I.

1908 Temperature coefficient of muscle contraction and surface tension is negative and of swelling positive. AGP cxxii I29, cxxiv 462.

I910 Thermo. currents of muscle. AGP cxxxi 589.

I9I2 "Elektrobiologie." Braunschweig. Vieweg.

I9I4 Muscle contraction. AGP clvi 299.

I9I5 Swelling causes no contraction unless previously stretched or twisted. AGP clxii I.

\& Von Tschermak I906 Electric organ. AGP cxii 439.

Bert, Paul I878 La pression barometique. Paris, Masson.

Berthelot \& Gaudechon I9Io Photosynthesis of carbohydrates. CRA cl I690.

\& Jungfleisch I872 Partition coefficient independent of concentration. ACP (4) $396,408$.

Bethe I908 Van't Hoff solution. Toxicity of sugar. AGP cxxiv 54I. Igog Movements of medusa and perm. to $\mathrm{H}^{\circ}$ and $\mathrm{OH}^{\prime}$. AGP cxxvii 2 Ig.

I9II Electroendosmose. MMW Iviii I68.

I9I4 Blood corpuscles of ascidians more permeable to acid than to basic dyes. BIOM (No. 284).

I9I6 Stimulation due to $\mathrm{H}^{\circ}$. AGP clxiii 147. 
- \& Toropoff $19 \mathrm{r}_{4}$ Change of reaction by electroendosmose. ZPkC $1 \mathrm{xxxviii} 686$.

Beutner 1912a Colloidal and osmotic swelling. BZ ix 280.

r9i2b Concentration cells. BZ xlvii 73.

I9I3a Concentration cells. ZEC xix 319.

19r3b Concentration cells. JPC xvii 344.

I9I3c Colloidal and osmotic swelling. BZ xlviii 217.

I9I3d Concentration cells. JACS xxxv 344.

r9I3e Concentration cells. AJP xxxi 343 .

I9I3f Concentration cells. TAES xxiii 40I.

Bialaszwicz 1908 Swelling of egg. BASC 783 .

$1912 \Delta$ of chicks and tadpoles. $\mathrm{AE}$ xxxiv 489.

Bigelow I 898 Negative catalysers. ZPC li 585 .

I907 Filtration same through porcelain and colloidal membranes.

JACS xxic 1675 .

\& Bartell Ig09 Pores in membranes. JACS xxxi II94.

Billard \& Bruyant 1905 Surface tension and locomotion. $C R B$ lix 102.

\& Dieulafe 1902 Surface tension of bile $=.66$. CRB liv 325 .

Billiter I9I3 Polyvalent ions and cataphoresis. APk (4) xi 902.

I904 Clearing of sols with sols of same sign. BWA cxiii II59.

I905 Surface tension and electrode potential. ZPkC li I29.

Biltz, H. 1899 Phototropism of dyes. ZPkC xxx 527.

Biltz, W. I904 Adsorption compounds of immune bodies. ZPkC xlviii 615 . I904 Colloidal hydroxides positive. BDCG xxxvii I095.

I9I3 Osmotic pressure of dextrin. ZPkC 1xxxiii 683.

- \& Steiner I9I0 Adsorption hydrolysis. KZ vii II3.

Bingham \& Durham IgI I Viscosity of suspensoid sols greater than water. ACJ xlvi 278.

Bjerrum I908 $\mathrm{KCl}$ lowers diffusion potential. ZPkC liii 428.

Bock \& Hoffmann I87I NaCl infusion causes glycosuria. AP 550 .

Bodenstein I9I3 Photochemistry. ZPkC lxxxv 329.

\& Dietz Ig06 Enzyme changes equilibrium point. ZEC xii 605.

Bodländer \& Fitting I902 Solubility of ammoniacal silver salts. ZPkC xxxix 597.

Böhm Ig09 Increase in blood pressure does not decrease volume. BZ xvi 3 I3.

Bohn, G. I904 Rhythm in littorina. CRA cxxxix 646. I907 Rhythm in diatoms. CRB 1xii I2I.

Bohr 1894 Secretion of gas into air bladder. JP xv 494. I904 Dissociation of oxyhemoglobin. ZP xvii 683.

\& Hasselbach I903 Calorimetry of embryos. SAP xxii 221.

Boldyreff Igr5 Regurgitation from duodenum to lower gastric acidity. QJEP vili $r$.

Bonnevie IgIo Mitosis. AZ v I.

Boothby I9I3 Anaesthesia. JPET v 379.

\& Peabody I9I4 Alveolar air. AIM 497.

Bordet 1899 Agglutinins do not agglutinate bacteria if ions are absent. AIP xiii 225 .

Boruttau I9I6 Ions and action current. ZP xxxi I.

Bose I9r3a Velocity of excitation impulse in plants. PT (B) cciv 63. I9x 3 b "Researches on the Irritability of Plants." Longmans.

Botazzi I $897 \Delta$ of marine animals. AIB xxviii 61 . I905 $\Delta$ of marine animals. AF iii 416 .

Ig09 Change of surface tensions of colloids. AF vii 593.

I9II Surface tension of lymph $=.89$. Neuberg's "Die Harn."

I9I3 Hemoglobin negative. AF xi 397.

\& Craifaleanu I916 PH of nerve juice $=6.04$, of grey matter juice $=6.12$. RRAL Xxv 73 . 
\& Ducceschi $1896 \Delta$ and buffer value of blood. AIB xxvi 16 I.

\& Enriquez 1901 Octopus gland in sea water absorbs water on stimulation. AIB xxxy I69.

Bournat Ig09 Effect of ions on electroendosmose. CRA cxlix 1366.

Bousfield IgI2 Ionic size. PRS (a) 1xxxviii I47.

\& Lowry I9Io Association of $\mathrm{H}_{2} \mathrm{O}$. TFS vi I $_{5}$.

Boveri 1908 Viscosity of blood. PrM lxiii 546.

Bovie I9r3 Coagulation by ultra violet light. S xxxvii 24.

I9I5 Direct reading potentiometer. JMR xxxiii 295.

Bradley rgıo Lipase. JBC viii $25 \mathrm{I}$.

I9I3 Enzyme synthesis. JBC xiii 407-43I.

— \& Taylor 1916 Gelatine abolishes latent period in autolysis. JBC $\mathrm{xxv} 363$.

Bredig 1894 Speed of organic ions. ZPkC xiii I9I.

I898 Metal sols. ZEC iv 5I4, 547.

I899 Acids and alkalis increase dissociation of ampholytes. ZEC vi 33.

I90I "Anorganische Fermente." Leipsic.

I907 Specific catalytic action of potassium bichromate. Rhythmic catalysis. BZ vi 283 .

\& Fajans I908 Asymmetric catalysis. BDCG xli 752.

\& Fiske 1912 Asymmetry and catalysis. BZ xlvi 7.

\& Weinmayr I903 Rhythmic catalysis. ZPkC xlii 602.

Brodie \& Vogt I9I0 Increased respiration of gut during resorption. JP $\mathrm{xl} 135$.

Brown, A. Ig09 Permeability of barley grain same as plasma membrane. PRS (B) 1xxxi 82 .

Brown, H. I9I4 Storage of oxygen by yeast. AnB xxviii 197.

Brown, O. Ig03 Fundulus egg protoplasm anodic. AJP ix III.

I905 Fundulus egg good insulator. AJP xiv 354 .

Brown, W. I9I5 Dilute alcohol increases permeability of collodion sacs. BJ ix 59I.

Brünings Ig03 Bioelectric currents. AGP c 367.

1907 Bioelectric currents. AGP cxvii 409.

Bürker I9Io Anodic oxidation of anaesthetic proportional to anaesthetic power. MMW lvii I443.

Buchner \& Rapp I899 Fermentation and oxidation. ZB xxxvii 82.

Bugarsky 1897 Calculation of $\mathrm{SO}_{4}{ }^{\prime \prime}$ from electrode potential. ZAC xiv $\mathbf{1} 45$. \& Liebermann 1898 Buffer value of proteins. AGP lxxii 51.

- \& Tangl I898 Conductivity of serum = .125 $\mathrm{n} \mathrm{NaCl}$. AGP lxxii 545.

Buglia $1907 \Delta$ of blood increased by passage through active muscle. BZ vi 158 .

I908 Maximum surface tension of emulsoids at isoelectric point. BZ xi 3 II.

Bunsen \& Roscoe I862 Photochemical law. APk cxvii 538.

Bunzel I9I4 Oxidase apparatus. JBC.

I9I6 Oxidases. JBC xxiv 9I, I03.

Burch 1892 Calibration of rapid excursion of capillary electrometer. PT (A) clxxxiii 8I.

Burdon-Sanderson 1882 Action current of plants. PT $\mathrm{r}$. I888 Action current of plants, PT clxxix (B) 417.

Burge I9I4 Oxidation of pepsin. AJP xxxiv 140.

Burian I9Ioa Bony fish secrete hypotonic urine. AGP cxxxvi 74I. rorob Ultrafiltration. ZP xxifi 767.

\& Drucker I9 Io Small thermometer for $\triangle$. ZP xxiii 772.

Burri \& Nussbaum I909 Surface tension of milk $=.685 . \quad$ BZ xxii go.

Burton I906 Speed of colloids in potential gradient same as ions. PM (6) $\mathrm{xi} 425$. 
I906 Electrolytes decrease speed of colloid particles. Effect of polyvalent ions. PM (6) xii 472.

Burton-Opitz I9II Viscosity of blood. JAMA lvii 353.

I9I4 On laking blood becomes more then less viscous. AJP $x \times x v 51$.

Busquet \& Pachon $1908 \mathrm{Ca}$ on heart. CRB lxv 599.

Buxton \& Rahe I909 Opposite sols precipitate only in equivalent proportions. JMR Xx II3.

\& Shaffer Ig06 Electrolytic solution tension and precipitating power. ZPkC lvii 47.

- Shaffer \& Teague Igo6 Reversal of charge of sols with sols of opposite sign. $\mathrm{ZPkC}$ lvii 47,64 .

Byers \& Walter I914 Electrolytic endosmose. JACS xxxvi 2284.

Callaud (\& Pelletier) I82I Chemiluminescence. JPm vii 579.

Calugareanu I9I0 Anaesthetics increase size of colloid particles by dissolving in them. BZ xxix 96 .

\& Henri I902 Salts diffuse from erythrocytes into isotonic sugar solution. CRB liv 210, 356 .

Campbell, Douglas, Haldane \& Hobson $1913 \mathrm{CO}_{2}, \mathrm{H}_{2}$ and respiratory center. JP xlvi 30 I.

Cannon I897 Movements of stomach. AJP i 359.

C \& Cattell I9I6 Action current of glands. AJP x1 I43.

Carlson 1906 Anesthetics increase rhythm. AJP xvii I82.

IgII Stretching nerve and conduction rate. AJP xxviii 323.

-, Greer \& Luckhardt $1908 \Delta$ of lymph and blood same yet lymph contains more colloids. AJP xxii 9I.

Orr \& Brinkman I9r4 Powlow-gastric juice. AJP xxxiii 86.

Carrell \& Guthrie I905 Transplanted kidney secretes hypotonic urine. S xxii 563.

Cary 19I6 Regeneration. JEZ xxi I.

Cathcart 1904 Serum antitryptic. JP xxxi 497.

Cernovodeanu \& Henri-1906 Bacteria are anodic. CRB 1xi 200.

Cesana I9I3 Ultramicroscopy of catalysis. AF xi I30.

Chambers 19I5 Microdissection. S xli 290.

Chapin $1902 \mathrm{CO}_{2}$ stimulates plant growth similar to ether. $\mathrm{F} \times \mathrm{xi} 348$.

Chiari 1909 Ether increases antolysis. AEP Ix 256.

IgI I Action of acids and alkalis on swelling of glutin, BZ xxxiii I67.

— \& Fröhlich I9II Vagus action on heart abolished with oxalates. AEP Ixvi ino.

\& Januschke I9II Formation of exudates inhibited by Ca. AEP Ix I20, WKW xxiii 427.

Chick \& Martin I9I2 Ppt. of heated proteins. JP xlv 26r.

Child rgl I Amitosis. BB xxi 280 .

I9I3 Cell respiration. JEZ xiv 153.

Choquard I9I3 Narcosis of fat and lean tissues. ZB Ix I.

Christianson, Douglas \& Haldane Igr3 $\mathrm{CO}_{2}$ from blood, JP xlvii (p ii).

Clark I899 In prolonged action of $\mathrm{Hg}$ salts ionization unimportant. JPC iii 263.

Clark, A. I9I3 Action of ions and lipoids on frog's heart. JP xlvii 66.

Clark, W. 19I5a PH of cultures. JBC xxii 87.

I9I5b $\mathrm{H}_{2}$ electrode. JBC xxiii 475 .

19r5c PH of modified milk. JMR xxxi $43 \mathrm{~T}$.

IgI5d PH of cultures. JID xvii IOg.

- \& Lubs I9 I5 PH of cultures. JID xvii I60. I9I6 Buffer mixtures. JBC xxv 479.

Clarke rgIr Analysis of sea water. BGS (no. 49I) II3. 1916 Ash of marine invertebrates. S xliii 723 .

Clausen 1890 Respiration of plants doubled by $10^{\circ}$ rise in temperature. LJ xix 893 . 
Clowes Ig03 $\Delta$ and sp. gr. of urine. AJP ix 3 I9.

I916 Soap gelation and permeability. PSEB xiii I-I4, JPC xx 407.

Coblentz I912 Temperature and spectrum of firefly. CIWP (No. I64).

Coehn I898 Suspensions of lower dielectric constant than water are anodic. WAP lxiv $2 \mathbf{2} 7$.

I909 Tyndall phenomenon of sugar solutions, ZEC xv 652.

- \& Barrat 1905 Galvanotropism. ZAP v I.

Cohnheim I 898 Very hypertonic solution in, gut draws water and salts out of blood. ZB xxxvi I29.

I90I Negative osmose through holothurian gut. ZPC xxxiii 9.

1902 Negative osmose through gut of octopus. ZPC xxxv $4 \mathrm{r} 6$.

19I3 Chopped kidney binds $\mathrm{NaCl}$ and sugar when it exceeds a certain concentration. ZPC lxxxiv $45 I$.

\& Von Uexknell I9I2 Catch-action of smooth muscle. ZPC lxxvi 314. Colton I9Io Surface tension. PANS 42.

Compton I915 Optimum PH for maltase 7.2 at $47^{\circ}, 3$ at $35^{\circ}$. PRS (B) Ixxxviii 408.

Conklin 1908 Movements in dividing eggs. Biol. Lect. Woods Hole. 69. I9I2 Cytoplasm nucleus ratio. JEZ xii $\mathrm{I}$.

Constantino 1914 Erythrocytes permeable to amino acids. AIB 1x 442.

Cooke I898 Increased osmotic pressure of tetanized muscle. JP xx I37.

Corral I9I 5 Dilution to $1 / 2$ does not change $\mathrm{PH}$ of blood. $\mathrm{BZ} 1 \times \mathrm{xxii} \mathrm{I}$.

Correns 1891 Turgor. JWB xxii I6r.

Craw I905 Toxin-antitoxin reaction. PRS (B) 1xxvi I79.

Cramer I9I5 Surface tension and metabolism. PRS (B) 1xxxviii 584.

Cremer I906 Concentration cells with membranes permeable only to $\mathrm{H}$ ions. ZB xlvii 562 .

Cumming \& Gilchrist I9I3 Diffusion potential in wide tube. TFS ix I74.

Curtis, H. A. - Non-inductive resistances. BBS (no. 3, 8).

Cushny I90I Diuresis and permeability. JP xxvii 429 . 1902 Blood pressure and diuresis. JP xxviii 443 .

Cybulski 1903 Membranes and concentration cells. BASC 622.

- \& Dunin-Borokowski Igog Effect of gelatine membrane on concentration cell. BASC 660 .

Czapek I902 Homogentisinic acid and geotropism. BDBG xx 464. I9I0 Plants killed by solutions of surface tension $=.68$. BDBG $\mathrm{xxviii} 159,490$.

Dahlgren 19I4 Origin of electric organ. CIWP (No. 183) 159.

Dakin, H. I905 Asymmetry and enzyme action. JP xxxii 199. I9I2 "Oxidations and Reductions in the Animal Body." Longmans.

Dakin, W. Ig08 Change of $\Delta$ of fishes. BJ iii 258 .

Dale rgr I Galvanotropism of infusoria. JP xxvi 29I.

Danysz I902 If antitoxin is added slowly more free toxin is left than if added suddenly. AIP xvi 331 .

D'Arsonval I889 Surface tension and bioelectric phenomena. AP +60 .

IgOI Resistance of bacteria to cold. CRA cxxxiii 84 .

Darwin, Francis 1903 Artificial rhythm in plants.

Davenport I897 Swelling of frog's embryos. PBS xxviii 73.

Davidsohn I9I2 Proteolysis in infants' stomachs. ZK iv 208.

I913a Methods for gastric analysis. Buffer value of stomach. ZK ix 470.

I9I3b $\mathrm{PH}$ and lipase. $\mathrm{BZ}$ xlviii 249.

Davies I907 Adsorption and solid solution. JCS ix I666.

Davis I9I5 Surface tension of $\mathrm{H}_{2} \mathrm{SO}_{4}$ solutions. Thesis, Columbia U.

Dawes I905 Adsorption of colloids irreversible. BCPP vi 426.

Deetjen I909 Alkalinity causes dissolution of blood platelets. ZPC lxiii I. Dekhuisen I904 $\Delta$ of fresh water animals. ANd $x$ I2r.

Delage IgO2 $\mathrm{CO}_{2}$ causes parthenogenesis of starfish. AZEG (3) $\mathrm{x} 2 \mathrm{I} 3$. I907 Parthenogenesis, AZEG xxxvi (N \& $R$ no. 2). 
DeMeyer I9II Swelling of sperm in egg extracts. AB xxvi 65 .

Demoor 1894 Asphyxia of cells. AB xiii I63. I907 Swelling and $\Delta$ of organs. AIP iv 340.

D'Errico $I 907 \Delta$ of fowl $=.66^{\circ}$. AF ix 453 .

Denham I908 Salt hydrolysis. TCS xciii 4I.

Denis I9I3 Fish blood and urine. JBC xvi 389.

DeVries I884 $\Delta$ of plant juices. Isotonic solutions. JWB xiv 427. I885 Permeability of vacuole membrane. JWB xvi 465 .

Dietz 1907 Catalysis in diphasic system. ZPC lii 279.

Dole I9I4 Analysis of sea water. CIWP (no. I82) $7 \mathrm{I}$.

Donaldson 1916 "The White Rat."

Donders 1872 Dissociation of oxyhemoglobin. AGP v 20.

Donnan I9II Osmotic pressure of Congo red. ZEC xvii 572.

\& Donnan 1905 Surface tension of urine, normal $=.9$, icterus $=.75$. BMJ 1636.

\& Harris I9II Osmotic pressure of Congo red. TCS xcix 1554.

\& Potts I910 Dispersion of soaps. Haptogen membranes and emulsions. $\mathrm{KZ}$ vii 208.

Douglass, Haldane \& Haldane I9I2 Dissociation of oxyhemoglobin. JP xliv 275.

Haldane, Henderson \& Schneider I9I3 Adaptation to high altitudes. PT (B) cciii I85.

Dreser I893 Toxicity and ionization. AEP xxxii 456.

Drew I9I4 Denitrifying bacteria in sea water. CIWP (no. I82) 9.

Dreyer \& Roy IgII Blood volume. PT (B) ccii I9I.

\& Walker I9I2 Resistance of dry bacteria to heat. JPB xvii $\mathrm{I}_{42}$.

Drury I9I4 Microchemistry of oxidation. PRZ (B) 1xxxviii I66.

Drucker I9I3 Calculation of $\mathrm{Ba}$ " from electrode potential. ZEC xix 804.

Dubois I9I3 "Mechanisme intime de la production de la lumiere chey les organismes vivants" (Soc. Linneenn de Lyon) (A. Rey).

DuBois-Reymond Resistance of muscle less in tetanus (II, i, 82). "Untersuchungen ueber tierische Elektricitaet." I9I6 Muscle contraction. BKW liii 393.

Duclaux I870 Surface tension curve. ACP (4) xxi 383, 427.

1905 Osmotic pressure of suspensoids. CRA cxl 1544.

1909 Osmotic pressure of colloids. JCP vii 405.

Dupré r866 Surface tension-adsorption curve. ACP (4) vii 409, ix 379.

Durig I90I Absorption of water by frogs. AGP 1 xxxv 401 .

Dutrochet 1835 Negative osmose. ACP (2) 1x 337.

Ebbecke 1894 Sudden increase of pressure to 300 atmospheres causes contraction of narcotized muscle. AGP clvii 79.

Edridge-Green I9I5 Color vision. JP xlix 265.

Ehrenberg I9I3 Lyotrope series in gelatine swelling depends upon concentration of salts. BZ liii 356 .

Ehrlich I885 "Das Sauerstoff-Bedürfniss des Organismus." Berlin, Hirschwald.

Einstein 1908 Brownian movement. ZEC xiv 235.

Einthoven Igor String galvanometer. ANd (2) vi 625.

Eisenberg \& Okoloska I913 $\mathrm{NaCl}$ increases toxicity of phenol. ZB lxix 312. - \& Volka I902 Adsorption of agglutinin by bacteria. ZH xl 154 .

Eisler 1909 Pure salt solution cause involution forms of bacteria. ZB (I) 1i 546 .

— \& Von Portheim Ig09 Al'" retards entrance of methyl violet into cells. BZ xxi 59.

Ellis, J. I9I6 Concentration (activity) of $\mathrm{H}^{\circ}$ in $\mathrm{HCl}$. JACS xxxviii 737. Ellis, R. I9I2 Effect of ions on oil sols. ZPkC lxxx 597 and lxxviii 321 . Embden \& Kraus 1912 Lactic acid produced by liver. BZ xlv I.

\& Kondo I9I2 Lactic acid production in muscle-press-juice inhibited by $\mathrm{H}^{*}$ (addition of alkali increases yield). BZ xlv 63 . 
Emmerling IgoI Specificity of enzymes. BDCG xxxiv 600, 2206, 3810.

Emslander I9I4 PH of liver. KZ xiv 44, xxiv 3810.

Endler 1912 Permeability of protoplasm. BZ xlii 440, xlv 402.

Engelmann I882 Phototropism. AGP xxix 387.

I893 Violin E-string swells and shortens in dilute acids or alkalis or on heating. "Ueber den Ursprung der Muslelkraft." Leipsic.

Engler \& Wild 1897 Peroxides and oxidation. BDCG $\times x \times 1669$.

Erlenmeyer 1913 Asymmetry and synthesis. BZ 1xiv 382.

Euler I907 Displacement of equilibrium point by enzymes. ZPC lii 146.

- \& Pope I9I2 "General Chemistry of Enzymes." N. Y. Wiley.

Erb Ig06 Adrenalin causes reduction of blood volume. DAKM lxxxviii 36.

Erlanger \& Garrey I9I4 Faradic stimuli. AJP xxv 337.

Enst Igor Temperature optimum for catalysis. ZPkC xxxvii 448.

Evans 1906 Adsorption of $\mathrm{NaCl}, \mathrm{KCl}$, etc. JPC x 290.

Evans \& Schuleman I9I4 Permeability to colloidal dyes. S xxxix 443.

Ewald I9I2 Reversal of phototropism by electrolytes. JEZ xiii 59r.

Ewart 1903 "Protoplasmic Streaming in Plants." Oxford, Clarendon Press.

Fahr 1909 Permeability of muscle. ZB lii (NF) xxxiv 72.

Fajans I9Io Asymmetry and catalysis. ZPC lxxiii 25, 1xxv 232.

Falk \& Nelson I912 Hydrolytic action of amino acids. JACS xxxiv 828.

Fallois Ig0I Increased oxygen pressure. AB xvii 713 .

Fano \& Botazzi I $896 \Delta$ of portal blood. AIB xxvi 45.

$\frac{1}{\text { F }}$ \& Mayer I907 Surface tension of serum $=.82$. AF iv 165 .

Faraday 1834 Catalysis by metals. PT. I857 Protection colloids. PT I54.

Farkas Igo3 PH of serum. AGP xcviii 55I.

\& Scipiades 1903 Blood during pregnancy. AGP $577,58 \mathrm{I}$.

Fenton I894 Oxidation of tartaric acid by iron. TCS 1xv 899.

Fernbach I 890 Alcohol increased permeability of fungi to enzymes. AIPr iv 672 .

I906 $\mathrm{PH}$ and diastase. CRA cxlii 285.

Field \& Teague I907 Immune bodies cathodic. JEM ix 86.

Fienga I9I0 Effect of salts on smooth muscle. ZB liv 230.

Findlay 1908 Adsorption of $\mathrm{CO}_{2}$ by sols. $\mathrm{KZ}$ iii 169 . I913 "Osmotic Pressure." Longmans.

Fischel Ig06 Granules and cell division. AE xxii.

Fischer, A. I895 Permeability of bacteria to salts. 1899 "Fixierung Färbung and Bau des Protoplasmas." Jena.

Fischer, E. I898 Asymmetry and enzyme action. ZPC xxvi 60.

- \& Abderhalden I907 Asymmetry and enzyme action. ZPC li 264.

Fischer, H. \& Jensen I909 Freezing out of colloids. BZ $\mathrm{xx}$ I43.

Fischer, M. 1908 Salts reduce the swelling action of acids and bases on proteins. AGP cxxv 99.

IgI4 "Oedema and Nephritis." and Ed. N. Y.

\& Moore 1907 Action of acids and alkalis on swelling of fibrin. AJP $\mathrm{xx} 330$.

\& Sykes I9I4 Alcohol and acetone retard swelling of gels. KZ xiv.

Fitting I906 "Reizleitungsvorgänge bei der Pflanzen." EP v 155. IgIr $\Delta$ of desert plants. $Z B$ iii 209.

Fitzgerald IgIo Location of $\mathrm{HCl}$ in gastric tubules. PRS (B) 1xxxiii 56.

Fleisher \& Loeb, L. I910 Absorption from serous cavities. JEM xii 288, 487.

Fleischhamer IgI3 Salts produce current of injury of heart. ZB 1xi 326. Fletcher 19I3 Lactic acid in muscle. JP xlvii 36I.

- \& Brown I9I4 $\mathrm{CO}_{2}$ production and beat rigor of muscle. JP xlviii I77.

- \& Hopkins 1907 Lactic acid is not burned by $\mathrm{O}_{2}$ if muscle is chopped up. JP XXxv 247.

Flexner \& Noguchi I906 Diffusion of colloids. JEM viii 547. 
Fluri I909 Polyvalent ions increase permeability of Spirogyra. F xcix 8r. Flusin Igo8a Negative osmose and swelling of membrane. ACP (8) xiii 480.

I908b Osmose and hydration of membrane. JPq (4) vii $29 \mathrm{I}$.

Forschhammer I865 Analysis of sea water. PT clv 203.

Forster 1873 Dogs die from lack of salt. ZB ix 297, 369.

Fowler, Bergeim \& Hawk 19I5 Indicators for stomach. PSEB xiii 58.

Fraenckel Ig04 Curve of conductivity of blood, and erythrocyte volume. ZKM lii (h5).

$1905 \mathrm{PH}$ of gastric juice. ZEP i r, 43I.

1907 Catalyser used up in catalysis. ZPC 1X 202.

Frankforter \& Kritchensky 1914 Catalysis. UMS (No. 2).

Franz, F. I903 Hirudin. AEP xliv 342.

Franz, V. Ig09 $\Delta$ of marine animals. IRGH ii 557.

Fredericq I904 $\Delta$ of shark changes with sea water. AB $\mathrm{xx} 709$.

Frenkel \& Cluzet I90I Surface tension of urine. JPP iii 99.

Freund $\mathrm{I}^{\mathrm{I}} 3 \mathrm{Na}$ favors and $\mathrm{Ca}$ inhibits fever. AEP 1 xxiv $3 \mathrm{Ir}$.

Freundlich 1903 Rate of addition of electrolyte to sol. ZPkC xliv 143 .

I907 Adsorption: formula, reduction of heavy metals by. ZPkC lvii I909 "Kapillarchemie." Leipsic, Akad Verlag.

I9I0 Adsorption, solid solution, potential and precipitating power. ZPkC lxxiii $385,397$.

I9r6 Negative osmose. Electrostenolysis. KZ xviii II.

\& Bjercke I916 Oxidation rate of oxalic acid by charcoal determined by diffusion. ZPkC xci 3 I.

\& Loser 1907 Adsorption hydrolysis. ZPkC lix 284.

\& Mäkelt Ig09 $\mathrm{E} \mathrm{M} \mathrm{F}$ produced by gravity or suspensions. ZEC xv I6I.

\& Neumann I909 Ionic exchange. Reversibility of adsorption. ZPkC Ixvii 538 .

\& Schucht I9I2 Precipitation of $\mathrm{As}_{2} \mathrm{~S}_{3}$-sol by ions. ZPkC 1xxx 564. I9I3 Adsorption and suspensoid precipitation. ZPkC 1xxxy 64I.

— \& Von Elissafoff I9I2 Adsorption potential of colloids. ZPkC lxxix 407.

Frey IgIo Reduction of $\mathrm{Cl}$ in blood by feeding $\mathrm{NaBr}$. ZEP viii. I9I2 Narcosis with ethyl chloride. BZ x1 29.

Friedemann \& Friedenthal I906 Albumins precipitated by nucleic acid. ZEP iii 73.

Friedenthal I900 Proteins and carbohydrates not absorbed from gut by lacteals. AP 252.

1903a Buffer value of serum. AP (physiol. Gesell. 1903).

I903b Indicators. ZEC x II3.

I903c Tensimeter. ZP xvii 437.

$1904 \mathrm{PH}$ of serum and indicators. ZAP iv 44.

I9II Significance of salts in milk. MMW 2385.

Frölich \& Meyer I912 Action-current of contracted muscle. ZP xxvi 269. Frozee I909 Electrical stimulation to growth. JEZ vii.

Fühner I9I2 Narcosis and evolution. ZB lvii 465.

— \& Neubaur IgO7 Hemolysis, $\mathrm{H}^{\circ}$ and $\mathrm{OH}^{\prime}$, and partition coefficient. AEP 1vi 333.

Gager 1908 Radium and growth. AN xlii 76 T.

Galeotti Igor $\mathrm{Na}$ antitoxic to $\mathrm{Cu}$ sol. $\mathrm{BC} \times x i$ 32I.

ro04 Electrodes for conductivity of tissue. ZB (nf) xxv 289.

1905 Water content of emulsoid particles. ZPC xliv 46r.

I 906 Bioelectric currents. ZAP vi 99.

Gallardo Ig06 Model of mitosis. CRA cxiii 228.

.'- Ganter IgI2 Temperature coefficients. AGP cxlvi I85. 
Gardiner, W. I884 Pores in plant cell walls. PT clxxiv 8I7.

1897 Surface movements of dividing eggs. Jour. of Morph. xi 55.

Garmus I9I2 Increased permeability of gland cells during activity. ZB lviii 185 .

Garten I906 Time of appearance of current of injury. ZP 673.

I909 Rhythmic response with single stimulus, ZB lii 534.

IgI I Electric organ. VGDN I.

Garrey I9I3 Resistance of fish to change in medium. AJP Xxxix $3 I_{3}$.

I9I 5 of animals and solutions. BB xxviii 77 .

Gaskell I 886 Positive variation of heart during vagus inhibition. JP vii $45 \mathrm{I}$.

Gassner 1906 Pos, and Neg. galvanopropism of roots. BZ lxiv I49.

Gasser \& Loevenhart I9I4 Respiratory center and asphyxia. JPET v 230.

Gatin-Gruzewska I904 $\Delta$ of glycogen $=0$. AGP ciii 282 .

\& Biltz I904 Ultramicroscopy of albumin. AGP cV II 5.

Gerassimow I902 Cell nucleus ratio. ZAP i 220.

Ghiron I9I3 Dyes filter through glomeruli. AGP cl 405 .

Gibbs $1874-8$ Surface tension and adsorption. TCA iii 380 .

Gibson \& Titherly 1908 Photoelectricity and photosynthesis. AB xxii 117.

Gildemeister I9I3 Polarizability of skin and psychogalvanic reflex. MMW 2389.

I915 Psychogalvanic reflex. AGP clxii 489.

Girard Ig08-I3 Negative osmose and electroendosmose. CRA cxlvi 927; cxlviii I047, I I86; cl I446; cliii 401 ; clvi I40I.

rgro Negative osmose and turgor. JPP xii $47 \mathrm{I}$.

I913 Hemolysis and electroendosmose. CRB lxxiv 520.

Glaser 1912 Calorimetry of embryos. S xxxv 189.

I9r3 Parthenogenesis. S xxxviii 446.

I9I4a Parthenogenesis. BB xxvi 387.

I9I4b Shrinkage of egg on fertilization. BB xxvi 84 . I9I5 Fertilization. BB xxviii I49.

Göthlin Ig02 Saline for frog's heart. SAP xii I.

Goldschmidt I899 Catalytic reaction in diphasic system. ZPkC xxxi 235 . \& Pribram roog Lipoids precipitated by anaesthetics. ZET vi I.

Goodridge \& Gies I9I I Edema. SEBM viii (106).

Gortner \& Harris I9I3 $\Delta$ of plant juices. BTBC xl 27. I9I4 Cryoscopic method. PW xvii 49.

Gotch \& Laws I885 Limulus blood ash. 54 Meeting Brit. Assn. 774.

Gouy I906 Lyotrope series and surface tension. ACP (8) ix 75.

Graham I854 Negative osmose of acids reduced by salts. PT cxliv I77. I86r Colloids and rate of diffusion. PT cli I83.

Gray I9I3a Increased conductivity of egg. PCPS xvii I.

I9I3b Increased conductivity of egg. JMBA $x 50$.

I9I5 Agglutination of sperm with ions. QJMS Ixi IIg.

Greely Igo4 Galvanotropism. JP vii 3 .

Greene $1904 \Delta$ of salmon. BBF xxiv 429.

rgog $\Delta$ of salmon. BBF xxix 129 .

rgro $\Delta$ of salmon. JEZ ix.

Griffiths 1892 "Physiology of Invertebrata." London.

Grijns I896 Hematocrit and osmotic pressure, AGP 1xiii 86.

Grode \& Lesser I9I3 Crushing liver greatly increases splitting of glycogen. $\mathrm{ZB} \mathrm{ix} 37 \mathrm{I}$.

Gros IgIO Adsorption of $\mathrm{NH}_{\mathrm{s}}$ and hemolysis. $\mathrm{BZ}$ xxix 350 .

I9I0 Neuraxons require 6 times as much anaesthetic as for general anaesthesia. AEP 1xii 379.

I9r2 Local anaesthetics are effective on all cells. AEP 1xvii r32.

Grünwald Igog Chloride starvation has same effect on nervous system as bromide poisoning. AEP $1 \times 360$. 
Grützner I893 Myelin sheath inhibits diffusion. AGP liii II5.

1894 Lyotrope series and excitability of nerve. AGP lviii 69.

Grumbach I9II Anesthetics and contact potentials. ACP xxiv 463.

Guldberg \& Waage I879 Law of mass action. ZPC cxxvii 69.

Gudzent Igoga Dissociation and isomerism of uric acid. ZPC $1 \mathrm{x} 25,38$; lxiii 253.

I909b Diffusible uric acid in serum. ZPC 1xiii 466.

I9I3 Colloidal solutions of uric acid. ZPC lxxxix 253.

Guthrie I9I4 Hypertonic laking. PSEB xi 149.

- \& Ryan I9I0 Action of Mg. AJP xxvi 329.

Guye I9I0 Association of $\mathrm{H}_{2} \mathrm{O}$. TFS vi 8.

Haber I908 Solution tension and polarization. APk (4) xxvi 927.

\& Klemensiewicz I909 Concentration cells with membranes permeable only to $\mathrm{H}$ ions. $\mathrm{ZPkC} 1 \mathrm{xvii} 385$.

Haberlandt I890 Geotropism of plants. BDBG' xviii $26 \mathrm{r}$.

Hägglund 19I5 Fatty acids inhibit yeast more than calculated from $\mathrm{PH}$. $\mathrm{BZ}$ lxix I8I.

Hagan \& Ormond I9I2 Vagus action on heart abolished with pure $\mathrm{NaCl}$ solution. AJP $\mathrm{xxx} 105$.

Haldane I892 Blood-gas apparatus. JP xviii 4I9.

- \& Priestly $1905.2 \%$ rise in alveolar $\mathrm{CO}_{2}$ doubles lung ventilation. JP xxxii 225 .

Halliburton 1916 Physiological Abstracts. London. i-

Hallwachs I888 Photoelectricity. APk xxxiii $30 \mathrm{I}$.

Hamburger I8go Plasmolysis. ZPC vi 3 II.

$1892 \mathrm{CO}_{2}$ causes $\mathrm{Cl}$ to pass from plasma into corpuscle. $\mathrm{ZB}$ xxviii 405. 1893 Hematocrit and osmotic pressure. ZP.

1896 Absorption from peritoneum increased by pressure. AAP 302. I898a Hemolysis with acids. AAP xxxi.

1898b Diffusible and non-diffusible alkali in serum. AB I.

I899 Protoplasm not a solution. AAP.

I902 "Osmotischer Druck und Ionenlehre in der Med. Wiss." Wiesbaden.

I908 Negative osmose through double membrane. BZ xi 443.

I9I2 "Untersuchungen ueber Phagocyten." Wiesbaden. Bergmann.

I9I5 Micro-analysis of $\mathrm{K} . \mathrm{BZ}$ 1xxi 415.

rgr5 Phagocytosis. IZPB ii $245,249,255$.

I9I6a $\mathrm{Ca}$, anesthetics, $\mathrm{CO}_{2}$ and lack of oxygen increase phagocytosis. BMJ 37.

I9I6b Erythrocytes permeable to $\mathrm{Na}$ and $\mathrm{K}$. WMM lxvi 521, 575.

\& de Haan I9Io Effect of salts on phagocytosis. BZ xxiv 304.

IOI3 Solubility, surface tension, toxicity and narcotic power. AAP 77.

\& Hekman I908 Phagocytosis. BZ ix 275.

\& Van Lier ${ }_{1902} \mathrm{CO}_{2}$ causes $\mathrm{NO}_{3}^{\prime}$ and $\mathrm{SO}_{4}^{\prime \prime}$ to pass into erythrocyte. AAP 492.

Hamill 1906 Antitrypsin. JP xxxiii 476.

Hammersten, Hedin \& Mandel I9I4 "Text Book of Physiological Chemistry." 7th Ed. N. Y.

Handowsky igro Hydrolysis of alkali albuminate. BZ xxv 5 IO.

I9I2 Partial hemolysis. AEP 1xix 4I2.

Harden \& McLean r9I I Cell oxidation. JP xliii $4 \mathrm{I} \%$

Hardy 1899 Structure of emulsoid coagulum and protoplasm. Precipitation of sols with $\mathrm{H}^{*}$. JP xxiv 158,301 .

I900a Solution. JPC iv 235, 254.

Igoob Isoelectric point $=$ least stability, and precipitation by opposite charge. $\mathrm{ZPkC}$ xxxiii 385 .

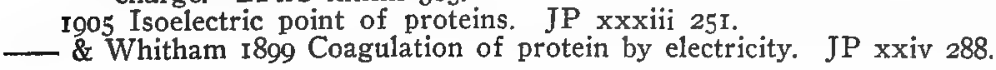


Harkins \& Humphrey IgI6 Stalagmometer corrections. JACS.

Harlow \& Stiles Igog Shaking enzymes, JBC vi.

Harris IgI4 Acid soils due to negative colloids. JPC xviii 355.

Harris, J. \& Gortner I9I4a Conversion table for $\Delta$. AJB i 75.

I9I4b Conversion table for $\Delta$. BCB iii 259 .

I9I5a $\Delta$ and conductivity of plant juices. BCB iii 196.

I9I5b $\Delta$ and conductivity of plant juices. $\mathrm{BCB}$ iv 52 .

Harrison I9Io Neuraxon growth and ameboid motion. JEZ ix 787.

Hartridge IgI2 Color vision. JP xlv (p. xxix).

Harvey IgI0a Fert. Mem. imperm. to sugar and proteins. JEZ viii 355 . 19Iob Parthenogenesis (Review). BB xviii 269.

I9I I Cell permeability. JEZ x 508 .

rgr2 Permeability of artificial cells. BCB ii 50.

I9I3 Indicator method and permeability. AJP xxxi 335.

I914a Fertilization membrane. BB xxvi 237.

I9I4b Permeability to acids. IZPB i 463.

I914C Permeability to alkali. CIWP (no. I83) I3I.

IgI5a Photogenesis. JACS xxxvii 396.

I9I5b Photogenesis. AJP xxxvi 230.

I916 Photogenesis. S xliv 208; 652.

Hasselbalch I9II $\mathrm{H}_{2}$-electrode. $\mathrm{BZ} \times \mathrm{xx} 317$.

I9I $2 \mathrm{PH}$ and respiratory center. $\mathrm{BZ}$ xlvi $4 \mathrm{I} 3$.

I9I3 Improved $\mathrm{H}_{2}$-electrode. $\mathrm{PH}$. I7 lower at $18.9^{\circ}$ than at $37^{\circ}$. BZ xlix 447, 45I.

\& Gammeltoft I9I5 PH of blood. BZ 1xviii 206.

- \& Lundsgaard I9I2 PH of blood. BZ xxxviii 77.

Hatschek I9I0 Ultrafiltration. KZ vii $8 \mathrm{I}$.

I9I I Viscosity of emulsoids due to swelling. $\mathrm{KZ}$ viii 34.

r9I3 Volume of disperse phase in emulsions. $\mathrm{KZ}$ xii 238.

Hauberisser \& Schoenfeld I9I3 Effect of salts on swelling of tendons. AEP lxxi I02.

Haycraft 1883 Hirudin. AEP xviii 209.

Heald I8g6 Electrolytic solution tension and toxicity. BZ xxii 125 .

Hedin I89I Hematocrit. SAP ii I34.

I895 Hematocrit and osmotic pressure. AGP 1x 360.

1895 Hematocrit and osmotic pressure. AGP Ix 360.

I898 Permeability of erythrocytes. AGP 1xx 525 .

1899 Rate of diffusion through dead gut. AGP Ixxviii 205.

1906 Enzyme action inhibited by adsorption of enzyme. BJ i 484 .

Ig09 Adsorption of colloids irreversible. ZPC $1 \times 364$.

Heidenhain I872 Blood pressure and formation of lymph. AGP v 309. I894 Negative osmose through gut wall. AGP lvi 579.

Heilbrunn I9I3 Membrane elevation due to decreased surface tension. BB xxiv 343 .

I9I5a Development and gelatin of egg. BB xxix I49.

I9I $5 \mathrm{~b}$ Oxidation in egg. $\mathrm{S}$ xlii $6 \mathrm{r} 5$.

Heimrod I9I3 Supposed catalytic action of oscillating magnetic field. ZAC xix 812 .

Hekma I9I5 Blood coagulation. IZPB ii 279, 299, 352.

Heller IgII $\Delta$ of blood rapidly increases after nephrectomy even in starving rabbit. ZP xxv 375 .

Hemptinne $1898 \mathrm{H}$ in atomic form in $\mathrm{Pd}$. ZPkC xxvii 429.

Henderson I907 Formula for diffusion potential. ZPkC lix is.

Henderson, L. Ig08 Buffers. AJP xxi r73.

Igog Dissociation of $\mathrm{CO}_{2}$. EP viii $254,274,3 \mathrm{I} 6$.

I9I0 PH of urine. $\mathrm{BZ}$ xxiv 40.

rgI I Kidneys regulate PH of blood. JBC ix 403.

I9I3 "The Fitness of Environment." Macmillan. 
\& Bloch 1908 Buffer value of $\mathrm{CO}_{2}$. AJP xxi 420 .

\& Spiro 1908 Buffer value of urine. BZ xv 105.

Henderson, V. I9Io Antitoxic action of sugar. ZP xxiv 5 I9.

Henderson, $Y$., Palmer \& Newburgh $\mathrm{H}^{*}$ and colloid swelling. JPET v 449. Henri 1904 Erythrocytes anodic. CRB lvi 866.

IgI I Photochemistry of sight. JPP xiii 84 I.

\& Lalou r903 $\Delta$ of shark changes with sea water. CRA cxxxvii 72 r.

- \& Mayer I904 Adsorption potential. CRA cxxix 974.

Henze I9I0 Increased $\mathrm{O}_{2}$ increases respiration of coelenterates. $\mathrm{BZ}$ xxvi 255.

I9I3 Blood corpuscles of acidians are acid. ZPC 1xxxvi 340.

Herbst I893 Artificial production of fertilization membrane. BZ xiii I4. $1898 \mathrm{OH}^{\prime}$ necessary for egg development. AE vii 486.

I904a $\mathrm{Na}, \mathrm{K}, \mathrm{Ca}, \mathrm{Mg}, \mathrm{Cl}, \mathrm{SO}_{4}, \mathrm{HCO}_{3}$, and $\mathrm{OH}$ necessary development of sea urchin egg. AE xvii 306 .

I904b Ca-free water causes cells to fall apart. AE xvii 440.

Herlitzka 19I2 Addition of urea, glycerine, arctameide, chloral hydrate or urethane improves Ringer's fluid. AF x 26I.

Hering $1915 \mathrm{KCl}$ stimulates vagus center. AGP clxi 537.

Herzog 1902 Zymase action is autocatalytic. ZPC xxxvii 149.

\& Betzel I9II $\mathrm{CHCl}_{3}$ and $\mathrm{AgNO}_{3}$ adsorbed by yeast but formaldehyde chemically combined. ZPC 1xxiv 22I.

\& Kasarnowski I908 Crystalloids diffuse more than I9 times as fast as colloids. $\mathrm{BZ} \times \mathrm{xi} \mathrm{I} 72$.

\& Meier I909 Asymmetry and enzyme action. ZPC lix 57.

I9I I Oxydases. ZPC Ixxiii 258.

Hess I9I3 Infant gastric juice. AJDC vi 264.

Heubner I905 Viscosity of blood. AEP liii 280.

Heymans I9I2 Permeability of filter ultrafilter and membranes to microbes. AIPd xxii 49.

Higgins I9I3 $\mathrm{CO}_{2}$ (Plesch, 20 sec.) $20 \%$ higher than Haldane. CIWP (No. 203) I7r.

\& Means I9I5 Alv. $\mathrm{CO}_{2}$ in acidosis, JPET vii I.

Hill, A. 1898 Synthesis of isomaltose. JCS 1xxiii 634.

I 903 Enzymatic splitting and synthesis of sugars. JCS lxxxiii 578 .

Hill, A. V. Igro Formula for Nernst's theory of stimulation. JP xl Igo. IgI2 Nerve impulse produces no heat. JP xliii 433.

I9r3a Half of heat of muscle produced during contraction; half during restitution. JP xlvi 28,435 ; xlvii 305 .

I9r3b Dissociation of oxyhemoglobin. BJ vii $47 \mathrm{I}$.

I9I4 Oxidation of lactic acid. JP xlviii (p. $\mathrm{x}$ ).

I9I5 Formula for blood-gas apparatus. JP 1 (vii).

\& Weizsäcker I9I4 Myothermic apparatus. JP xlviii (p. xxxy).

Hill, L. \& Nabarro 1895 Not always more $\mathrm{CO}_{2}$ in venous blood from active organ (vascular dilatation). JP xviii 220.

Hirokawa I908 Swelling of pores of kidney due to urine. AF xi 458 . Igog Effect of salts on spermatozoa. BZ xix 291.

Hirschfeld $1907 \mathrm{HgCl}_{2}$ may lake erythrocytes. AH lxiii 275. 1909 Ameboid motion. ZAP ix 529.

Hirschfelder IgI I Electrocardiogram. Inters. Med. J1. xviii (6).

His \& Paul I900 Dissociation of uric acid. ZPC xxxi I, 64.

Höber I899 Rate of diffusion and adsorption by gut. AGP lxxiv 225, 246. I900 PH of blood. AGP Ixxxi 522.

Igoza Selective adsorption of iron by gut (other heavy metals absorbed less). AGP xciv 337.

1903b PH of blood. AGP xcix 572.

I904a Erythrocytes and yeast are anodic; changed by polyvalent ions, impermeable to $\mathrm{Na}^{\circ}, \mathrm{K}^{\circ}, \mathrm{NH}_{4} ; \mathrm{Ca}^{*}$ and $\mathrm{Mg}^{\circ i}$. AGP ci 607 , cii I96. 
I904b $\Delta$ of fresh water animals. AGP cii 199.

I905 Alkali salts increase permeability of muscle. AGP cvi 599.

I907 Current of injury reduced by anaesthetics but caused by toxic dose of anaesthetics. AGP cxx 492.

1907 Inverse lyotrope series for positive and negative proteins and irregular series for neutral proteins. Series for lecithin. BCPP xi 35.

I908a Hemolysis and lyotrope series. BZ xiv 209.

I908b Physikalische Chemie d. Blutes und. Lymphi. Oppenheimer's Handb. d. Biochem. II 2, I.

Igoga Theory of skin current. ZEC xv 519.

I90gb Effect of salts on cilia. BZ xvii 5 I8.

I $909 \mathrm{c}$ Dispersion of dyes. $\mathrm{BZ} \times \mathrm{xx} 56$.

Igogd Permeability of erythrocytes. ZPC 1 $\mathrm{xx}$ I34.

Igroa Conductivity of cell interior. AGP cxxxiii 237.

I9rob Effect of organic anions on cells. AGP cxxxiii 3II.

I9IOc Alkali salts increase permeability of muscle. AGP cxxxiv 3 I6. Igrod Stimulation. ZAP x (Ref.) I73.

I9I2a Lung is permeable to $\mathrm{NH}_{3}$. AGP cxlix 87.

I9I2b Conductivity of cell interior. AGP cxlviii I89.

I9I3a Muscle contraction. ZEC xix 738.

I9r3b Increase of conductivity of blood with increased alternations of current. Cond. of interior of muscle fibre. AGP cl 15.

I9r3c Bleaching of wool-violet by protoplasm. ZB xlviii 291 .

I9I4 "Physikalische chemie der Zelle und der Gewebe." 4 th ed. Leipsic.

\& Jankowsky 1903 PH of urine. BCPP iii 525.

\& Kempner 1908 Kidney cells absorb all dyes but suspensoids. BZ xi 105.

\& Königsberg I905 Dyes excreted by frog's kidney carried in preformed granules. AGP cviii 323 .

\& Nast 19.14 Saponin hemolysis and lyotrope series. BZ $1 x$ I3I.

\& Spaeth I9I4 Rare earths on muscle. AGP clix 433.

\& Waldenberg 1909 Effect of organic ions on cells. Position of Cs in lyotrope series. AGP cxxvi 33I.

Hoffmann I889 Buffer value of proteins. ZIM $x 793, x 521$.

I9I4 Haptogen membranes and emulsions. Collection of particles at surface. ZB 1xiii 386.

Hofmeister I888 Hofmeister series. AEP xxiv 247.

I89I Hofmeister series. AEP xxviii 210.

Hoitsema 1895 Hydrogen in atomic form in Pd. ZPkC xvii $\mathrm{I}$.

Hooker I9II Ions and $\mathrm{CO}_{2}$ on vascular tone. AJP xxviii $36 \mathrm{I}$.

IgI2 Effect of $\mathrm{CO}_{2}$ and $\mathrm{O}_{2}$ on vascular tone in the gut. AJP xxxi 47.

Howe \& Hawk I9I2 PH of feces. JBC xi 129.

Howell I9I4 Blood coag. AJP xxxv I43, AIM xiii 76 .

\& Duke I908 Vagus inhibition and $\mathrm{KCl}$. AJP xxi $5 \mathrm{I}$.

Howland \& Marriott I9I6a Acidosis and diarrhea. AJDC xi 309. I9I6b Alv. $\mathrm{CO}_{2}$ and acidosis. JHHB xxvii 63 .

Hoyler 1907 Autocatalytic action of $\mathrm{H}:$ ZPC 1404.

Hudson I908 Invertase action. JACS $\mathrm{xxx}$ II60, I564. IgIo $\mathrm{PH}$ and invertase, JACS xxxii 1220 .

Hüfner 1903 Dissociation of oxyhemoglobin. AAP 2I7.

- \& Gansser Ig07 Osmotic pressure of hemoglobin. Mol. wt. = I632I. AAP 209 .

Huenekens I9I4 Effect of diet on PH of infant's stomach. KZ xi 297.

Hughes, A. L. I9I4 "Photo-Electricity." Cambridge U. Press.

Hulett Igor Surface tension and solubility. ZPkC xxxvii 385. I903 Osmotic pressure and capillarity, tensile strength of water. $\mathrm{ZPkC}$ xlii 353. 
Hutchinson I9I5 Resistance of Paramecium. JEZ xix 21I.

Hyde 1904 Action current of cell division. AJP xi 52.

- \& Spreier 19I5 Light and development. AJP.

Hyman I9I6 HCN and oxidation. AJP x1 238.

Iscovesco rgroa Catalase cathodic. Pepsin anodic. BZ xxiv 53.

I9I0b Surface tension of emulsions. CRB lxix 537.

I9IOC Cholesterin inhibits action of soap and lecithin by combining with them. CRB 1xix 566.

Ishizaka \& Loewi Ig05 Ca antitoxic to muscarin. ZPk xix 593.

Itami \& Pratt I909 Oxidation and cell structure. BZ xviii 303.

Jackson I9I5 Growth of organs of rat. JEZ xix 99, AJA xviii 75.

Jacobs 1909 Dessication of rotifers. JEZ vi 207.

Jacoby I9Io Enzymes of egg and sperm. BZ xxvi 336, 536.

- \& Golowinski 1908 Precipitate of caffein in muscle. AEP lix (sup) 286.

Jahnson-Blohm I9r3 Enzymes displaced from surface by anaesthetics and saponin; and activity restored by the latter. ZPC 1xxxii I78.

Januschke I9I3 Formation of exudates inhibited by anaesthetics. WKW (No. 20).

Jennings Ig04 Tropisms. CIWP (No. 16).

I906 "The Behavior of the Lower Organisms." Columbia U. Press.

1914 Paramecium swims backward consinuously in stimulating solution. JCNP xiv 442.

Jensen \& Fischer I9I3 Hydration in muscle. ZAP xiv 320.

Joel I9I5 Anaesthetics retard increase in conductivity of erythrocytes. AGP xlxi 5 .

Johannsen 1906 Etherization causes lilacs to sprout earlier. "Das Aetherverfahren beim Fruehtreiben." Jena.

Johnston $1916 \mathrm{CO}_{2}$ in water. JACS xxxviii 947.

Jones, H. I897 "Freezing Pt., Boiling Pt. and Conductivity Methods." Easton, $\mathrm{Pa}$.

1907 Hydrates in solution. CIWP (No. 60).

Jones, W. I9I3 Surface tension of solids. ZPkC lxxxii 448.

Jordan 1913 "Vergleichengle Physiologie Wirbelloser Tiere." Jena.

Jordis 1908 Colloids are electrolytes. KZ ii $36 \mathrm{I}$, iii 13 .

Jorissen \& Woudstra I9I I Beta rays coagulate positive sols. KZ viii 8.

Joseph \& Meltzer IgI I Abolition of irritability with $\mathrm{Ca}$ and restoration with Na. AJP xxix I.

\& Austin Igoo Toxicity and ionization. JPC iv 76.

- \& True I89 Toxicity of Cu ions. BG xxii $8 \mathrm{I}$.

$1896 \mathrm{n} / 6400 \mathrm{H}^{\circ}$ toxic. JAMA xxvii I38.

Kanda 19I4 Reversibility of geotropism. AJP xxxv I62. I9I6 Geotropism. BB xxx 57.

Kanitz I903 $\mathrm{H}^{\circ}$ and invertase. AGP c 547. I906 Dissociation of amino acids. ZPC xlvii.

I907 Dissociation of tyrosine and phenylalamine. AGP cxviii 539.

I9I5 Temp. Coef. of respiration. IZPB ii 272.

Kastle I9I0. Oxidases. BHL (No. 59).

\& Loevenhart Ig0o Synthesis of fat by pancreas lipase, ACJ xxiv 49I.

I903 HCN may hasten catalysis. ACJ xxix 397.

1907 Synthesis of fat by pancreas lipase. JBC ii 427.

Katy 1896 Salts in frog's muscle. AGP lxiii $\mathrm{r}$.

Katzenellenbogen 1906 Permeability of the gut. AGP cxiv 522.

Kaufler 1903 Surface tension and osmotic pressure. ZPkC xliii 686.

Keith, A. \& Flack 1907 Conduction in heart. JAP xli 172.

Keith, N., Rowntree \& Garaghty I915 Blood volume. AIM xvi 547.

Kidd ${ }_{1916} \mathrm{CO}_{2}$ inhibits cell respiration. PRS (B) lxxxix 136 . 
Kirsch IgI2 Surface tension of solutions increasing permeability of yeast to invertase $=.5 . \mathrm{BZ} \times 1$ I52.

Igr2 Surface tension of cells. BZ x1 152 .

Kite I9I3 Permeability of protoplasm. BB xxy I.

Knoblach igoI Charge of colloids. ZPkC xxxix 225.

Knüpffer \& Bredig I898 Temperature and direction of reaction. ZPkC xxvi 255 .

Knowlton I9II Addition of gelatine to blood decrease secretion of urine. Starch solution not effective. JP xliii 2 I9.

Koch \& McLean 1910 Anaesthetics and size of lipoid particles. JPET ii 249 .

Kodis 1898 Undercooling of muscle. ZP xii 593.

I90I Conductivity of dying muscle. AJP $v 26 \%$

Köhler 1904 Ultra violet microscopy. ZWM xxi I29, 273.

Koelichen Igoo Polymerization in concentrated solution only. ZPkC Xxxiii I29.

I895 Hematocrit and osmotic pressure. AAP I54.

Koeppe I897 Erythrocytes increase ionization of $\mathrm{CO}_{2}$ and $\mathrm{HCO}_{3}^{\prime}$ exchanged for $\mathrm{Cl}^{\prime}$ of serum. AGP lxvii 189 .

Ig0o Physikalische Chemie in der Medizin. Wien.

1905 Erythrocytes in hematocrit appear laked. AGP cvii I83, 187.

Kohlrausch 1907 Speed of ions. ZEC xiii 333.

\& Heydweiller 1894 Conductivity of $\mathrm{H}_{2} \mathrm{O}=.0000385$ at $18^{\circ}$. ZPkC xiv 3I7; APk liii 209.

Koltzoff 1908 Swelling of spermatozoa. AZ ii I.

I9I2 Effect of salts on contractile stalk of Zoothamnium. AGP cxlix 327.

I9I4 Optimum PH for phagocytosis. JZPB i 82.

Koopman 19I5 Enzymes. JZPB ii 266.

Konikopf I9I3 PH of blood. BZ li 200.

Koranyi, Von $1897 \Delta$ of blood varies with $\mathrm{CO}_{2}$. ZKM xxxiii I.

Kovacs I902 $\Delta$ of blood varies with $\mathrm{CO}_{2}$. BKW xxxix 362 .

Kozawa IgI4a Hemolysis greatest at isoelectric point. Reversal of cataphoresis. $\mathrm{PH}$ of $\mathrm{H}_{2} \mathrm{PO}_{4}+\mathrm{NAH}_{2} \mathrm{PO}_{4} \cdot \mathrm{BZ} 1 \times{ }_{146}$

I9I $4 \mathrm{~b}$ Permeability of erythrocytes to sugar. $\mathrm{BZ}$ IX $23 \mathrm{I}$.

Krafft 1896 Soaps are colloids. BDCG xxix 1328 .

Kreibich I9I0-I $\mathrm{Hg}$ does not markedly affect PH of blood. WKW xxiii 355 xxiv.

Krogh $1904 \mathrm{CO}_{2}$ tension of sea water. Meddelelser om Grönland, xxvi 333. Copenhagen.

Ig08 Micro-tonometer. SAP $\times x$ 259, 279.

I9Io Absorption of $\mathrm{O}_{2}$ and $\mathrm{CO}_{2}$ by water. SAP xxiii 224 .

Igro Blood gas exchange in lungs. SAP xxiii 248 .

I9I4 Temperature coefficient of development. ZAP xvi 163, I78.

\& Krogh $1910 \mathrm{CO}_{2}$ and $\mathrm{O}_{2}$ tension of arterial blood. SAP xxiii 179.

Kuehne I864 Violet cells of Tradescantion become red at anode and green at cathode (due to metal electrode). "Untersuchungen ueber das protoplasma und die Contractilitaet." Leipsic.

Küster, E. I9I0 Formation of plasma membrane. ZBt ii 689; AE xxx 35 I. I9II Plant cells absorb many sulfonic acid dyes. JWB $126 \mathrm{I}$.

I9I3 Liesegang's rings. $\mathrm{KZ}$ xiii 192.

Küster, F. I894-5 Adsorption formula. ZPkC xiii 445; AC cclxxxiii 360. Kyes I902 Cobra hemolysin activated by lecithin. BKW 886.

Lachs \& Michaelis I9II Adsorption of $\mathrm{NaCl}$ not antagonized by nonelectrolytes. $\mathrm{KZ}$ ix 275 ; ZEC xvii I.

Landolt-Boernstein-Roth Igr2 Physikochemische Tabellen. 4th Ed. Berlin. Landsteiner 1913 Blood shadows precipitate at isoelectric point. BZ 1 176. 
— \& Jagec I904 Precipitation of emulsoids with suspensoids; adsorption compounds of immune bodies. MMW (No, 27).

\& Uhlirz Ig05 Adsorption of colloids irreversible. ZB x1 265.

Lapique I909 Nernst's theory of stimulation. CRA cxlix 871; JPP xi 1009, 1035.

\& Legendre 1913 Diameter of neuraxon and conduction. CRA clvii II63.

Laquer \& Sackur Ig03 Viscosity of casein increased by acids and alkalis. $\mathrm{BCPP}$ iii 193 .

Leathes I895 $\Delta$ of lymph greater than blood. Infusion of hypertonic $\mathrm{NaCl}$ increases blood volume. JP xix I, $4 \mathrm{I} 8$.

LeBlanc I9I3 No Tyndall phenomenon of sugar solutions. ZEC xix 794. Lee 1903 Alcohol increases rhythm of medusa. AJP viii (p. xix).

- \& Salant Ig02 Alcohol and muscle. AJP viii 6I.

Lehmann 1884 Mice and frogs die in compressed oxygen. Oxidation of pyrogallol independent of $\mathrm{O}_{2}$ pressure. AGP xxxiii.

Lepeschkin I908 Light increases permeability of plant cells. BDBG xxvi (a) I98, 23I, 724.

I91o Plasma membrane and Traube's membrane are sols. BDBG xxviii 383 .

I9II Composition of plasma membrane. BDBG xxix 247.

Lesser I907 Effect of temperature on skin current. AGP cxvi I24.

Levy, Rowntree \& Marriott I9I5 Indication method for blood. AIM xvi 3.

Levy \& Rowntree I9I6 Buffer value of blood. AIM xvii 525 .

Lewis, G. Ig06 Color change of $\mathrm{Co}$ or $\mathrm{Cu}$ solutions due to hydration of ions. $\mathrm{ZPkC}$ lvi 223 .

$1908 \mathrm{P}=12.06 \Delta-.02 \mathrm{I} \Delta^{2}$ JACS $\mathrm{xxx} 668$.

- \& Keyes 1912 Sol. Ten. of $\mathrm{K}$ and $\mathrm{Li}$. JACS xxxiv I22.

- \& Lacey I9I4 $\mathrm{E}_{\text {н }} \mathrm{Cu}=-.3347$. JACS xxxvi 804 .

— \& Randall 1914 Free energy of H. JACS xxxvi 1969.

- \& Rupert I9II Solution tension of C1. JACS xxxiii 299.

Lewis, M. I9I6 Sea water as culture medium. AR x 287.

Lewis, W. 1908 Haptogen membranes. PM (6) xv 499.

I909 Surface tension between oil and water. PM (6) xvii 466. Electric charge of oil drops in water. $\mathrm{KZ}$ iv $2 \mathrm{II}$.

I9Io Surface tension between liquids, adsorption of $\mathrm{NaCl}, \mathrm{ZPkC}$ Ixxiii 129 .

Lewites Ig07 Swelling of gels. KZ ii 166.

Liebreich I890 Surface films and chemical reaction. ZPkC v 529.

Liesegang, R. I9II Liesegang's rings. AE xxxiii 328.

L’Hermite 1855 Permeability by solution in membrane. ACP (3) xliii 420. Lillie, F. I9I3 Acids cause aggregation and alkalis agglutination of sperm. JEZ xiv 515.

Lillie, R. S. I902 Oxidation at nucleus. AJP vii $4 \mathrm{I} 2$.

I903 Spermatozoa are electronegative. AJP viii 273.

1905 Model of mitosis. AJP xv 46.

1907 Osmotic pressure of proteins reduced by salts and increased by $\mathrm{H}^{*}$ and $\mathrm{OH}^{\prime}$. AJP $\mathrm{xx} 127$.

I908 Heat-Parthenogenesis. JEZ v 375.

Ig09a $\mathrm{H}^{\circ}$ produces polarization of plasma membrane. Lyotrope series and cytolysis, $\mathrm{MgCl}_{2}$ decreases permeability. AJP xxiv I4, 36 . Ig0gb Artificial parthenogenesis and permeability. BB xvii I88.

Igogc Effect of salts on cilia. AJP xxiv 459.

I9I0 Lyotrope series, cytolysis and parthenogenesis. AJP xxvi ro6, I26.

Igrra Permeability, stimulation and contraction. AJP xxviii 197.

I9IIb Fertilization. BB xxii 328. 
I9I2a Anaesthetics may increase size of lipoid particles in plasma membrane by dissolving in them. AJP $x \times x 372$.

I9I2b Anaesthetics decrease toxicity of pure NaCl. AJP xxx I.

I9I3a Anaesthetics inhibit increase in permeability. AJP xxxi 255.

Igr $3 \mathrm{~b}$ Oxidation in blood cells increased by induction shocks. JBC $\mathrm{xv} 237$.

I9r3c Cell division. JEP xv 23.

I9I4a Rate of conduction. AJP xxxiv 4 I4.

I9I4b Anaesthetics vs. salts. JEZ xix 59I.

I9I4C Cell narcosis. JBC xvii I2I.

r9I4d Stimulation and permeability. PSM 579.

I9I6a Fertilization increases permeability of egg to water. AJP xl 249. $1916 \mathrm{~b}$ Rate of nerve impulse varies as electric conductivity of medium. AJP xli I26.

I9I6c Anaesthesia. BB xxx 3II.

Linder \& Picton 1895 Antagonism of cations in precipitation of sols. Electric charge, dispersion and freezing out of colloids. JCS lxvii $63,73$.

I897 $\mathrm{Al}_{2}(\mathrm{OH})_{6}$ and $\mathrm{Fe}_{2}(\mathrm{OH})_{6}$ sols are electro-positive. Conductivity of suspensoids same as water. Mutual precipitation of sols of opposite sign. JCS 1xxi $568,572$.

I905 Ultramicroscopy of coagulation. JCS lxxxvii I9II.

Lipscomb \& Hulett I9I6 Electrolytic calomel. JACS xxxviii 20.

Locke 1900-I Locke's solution. ZP xiv 670, xv 490.

Lodholz I9I3 All or none law. ZAP xv 269.

Loeb, J. I889 Heliotropismus der Tiere. Würzburg.

I897 Stimulating current must run longitudinally in nerve. AGP lxvii 483 , Ixix 99.

I $898 \mathrm{a}$ Swelling of muscle. AGP Ixix I.

I898b OH' necessary for egg development. AE vii $63 \mathrm{r}$.

I900 Artificial parthenogenesis. AJP iii 135.

1902 Toxicity of $\mathrm{Na}^{*}$ and antitoxin action of bivalent ions. AJP vi $4 \mathrm{II}$.

I903 Toxicity of sugar. AGP xcvii 394.

I906 $\mathrm{Ca} *$. Sr" and $\mathrm{Ba}^{*}$ cause rhythmic contraction of center medusa. JBC i 427.

1907 Antitoxic action of $\mathrm{Ca}^{\bullet}$ on $\mathrm{Na}^{\circ}$ in cytolysis of sea urchin eggs. $\mathrm{BZ} \vee 35 \mathrm{I}$.

I9I1a Fundus eggs live in distilled water. AE xxxi 654.

IgI Ib Antitoxic action of $\mathrm{Ca}^{*}$ and $\mathrm{Sr}{ }^{*}$ on $\mathrm{K}^{\circ}$ and $\mathrm{N}_{2}{ }^{\circ}$ and of $\mathrm{Na}^{\circ}$ on $\mathrm{K}: \mathrm{BZ}$ xxxi 450 ; $x \times x i i \mathrm{I} 55,308$.

IgIza Toxicity of sugar. JBC xi 4I5.

I9I2b Increased permeability to water. BZ xlvii I27.

I913 Artificial Parthenogenesis and Fertilization. Chicago.

I9I4a "Fertilizin" not necessary for fertilization. JEZ xvii I23.

I9I4b Bunsen-Roscoe law and phototropism. ZP xxvii 80.

I9I5a Salts antitoxic to acids. JBC xxiii I39.

I9I $5 \mathrm{~b} \mathrm{Ca}$ and permeability. JBC xxiii 423 .

I9I6 Phototropism. JEZ xx 2I7.

\& Budgett 1897 Galvanotropism. AGP 1xv 5 I8.

\& Beutner 19I2 Current of injury in plants. BZ xli I, xliv 303.

I9I3 Current of injury. BZ li 288 .

I9I4 Bioelectric currents. BZ lix 201 .

— \& Cattell I9I5 Antagonism of salts. JBC xxiii 4I.

— \& Ewald I916 Chemical stimulation of nerve. JBC xxv 377.

—_ \& Wasteneys I9I0 Oxidation in egg. Medusae anaesthetized by $\mathrm{Mg}$ use more $\mathrm{O}_{2}$. $\mathrm{BZ}$ xxviii 340,350 . 
IgI Ia Bases and alkaline salts increase oxidation. $\mathrm{BZ}$ xxxviii $4 \mathrm{IO}$.

IgI Ib Bromide intoxication of fish. BZ xxxix I85.

Igrza Adaption to heat. JEZ xii 543.

1912b Heart rhythm reduced by reduction of $\mathrm{O}_{2} . \quad \mathrm{BZ} \times 1277$.

I9I3 Anesthetics reduce cell division more than oxidation. JBC xiv 517.

I9I5a Effect of poisons on $\Delta$ of Fundulus. JBC xxi 223.

I9r5b Osmotic pressure of cell. JBC xxili I57.

I9I5c Phototropism. JEZ xix 23.

Loeb, L. Igor Phagocytosis. AGP cxxxi 465.

Loeb, W. \& Higuchi I9Io PH of serum of placenta. BZ xxiv 92.

Loew I889 Synthesis of sugar from formaldehyde. BDCG xxii 470.

1892 Antagonism of $\mathrm{Ca}$ and $\mathrm{Mg}$. F 368 .

Loewe, F. I9I2 Water interferometer. $\mathrm{KZ}$ xi 226.

Loewe, S. Igr2 Methylene blue adsorbed by lecithin (in chloroform), BZ xlii $150-218$.

I9I3 Anesthetics reduce conductivity of artificial lipoid membranes. $\mathrm{BZ}$ lvii I6r.

Loewy 1894 Alkali albuminate more concentrated in corpuscle than in serum. AGP lviii 462.

\& Zuntz I894 $\mathrm{CO}_{2}$ increases diffusible alkali in serum. AGP lviii 5 II. Loomis $1894 \Delta$ of $\mathrm{NaCl}$. APk li 5 I5. I897 $\Delta$ of $\mathrm{NaCl}$. APk ix 527 .

- \& Acree I9I I Calomel electrode. ACJ xlvi 585.

Lorant I9I4 Surf.-Ten. between Liquids. AGP clvii 2II.

Lorenz \& Boehi Igog Dissociation of water. ZPkC 1xvi 749.

Lottermoser 1907 Adsorption by filters. ZPkC $1 \times 458$.

I908 Freezing out of colloids. BDCG xli 3976.

rgro Peptization and reversal of charge of sols. $\mathrm{KZ}$ vi 78 .

— \& Rothe I908 Impurities of sols necessary for stability. ZPkC 1xii 359.

Löwenherz I896 Influence of alcohol on dissociation of water. ZPkC $\mathrm{xx} 283$.

Lubs \& Clark I9I5 New indicators. JWAS v 609.

Lucas Igoga Temperature coefficient of nerve conduction. JP xxxvii II2. $1909 \mathrm{~b}$ Rise of neg. var. prop. to rate of transmission. JP xxxix 207. Igogc All or none law. JP xxxviii II3.

Ludwig 1849 Swelling of colloids. ZRM viii.

Ludloff I895 Galvanotropism due to reversal of cilia at cathode. AGP lix 523 .

Lunden 1908 Ampholytes. JBC iv 267.

Lundsgaard I9I2 PH of blood. BZ xli 247.

Lusk I9I2 "The Elements of the Science of Nutrition." 3rd Ed. Phila. r9I5 Influence of food on metabolism. JBC xx 555 (and p. viii).

Luther 1896 Adsorption potential of colloids. ZPC xix 529.

\& Forbes I909 Photochemistry. JACS xxxi 770.

Lyon I898 Otocyst. JCNP viii 238.

Igor Rhythmic production of $\mathrm{CO}_{2}$ and sensibility to heat and cold (eggs). AJP xi 52.

1902 Cell division and rhythmical susceptibility to KCN. AJP vii 56 . rgo3 Artificial parthenogenesis. AJP ix 308.

1904 Rheotropism. AJP xii 147.

1905a Geotropism. AJP xiv 42r.

I905b Density of eggs. AJP xxiv 244.

I906 Geotropism. BB xii 2 I.

Ig09a Rheotropism. AJP xxiv 244.

roogb Catalase and fertilization. AJP xxv I99. 
\& Shackell I9Ioa Autolysis and fertilization. JBC vii 37 I.

IgIob Fertilization and permeability. S xxxii 249.

Macallum I904 Blood salts same as Cambrian sea. Trans. Can. Ins. 1905 Microanalysis of $K$. JP xxxii 95.

Igo8 Microanalysis. EP vii 552.

IgI I Surface tension. EP xi 595.

I9I2 Surface Tension and Vital Phenomena. Toronto Univ. Press (Physiol.) No. 8.

I9r3 Muscular energy. JBC xiv (p. ix).

\& Benson Igog $\Delta$ of urine may be as low as .0\%. JBC vi 87.

McCallum I905 Regeneration in plants. BG.

McCaskey I908 Viscosity of blood. JAMA li I653.

McClendon I907 Removal of chromatin from eggs with "mechanical hand." $\mathrm{BB}$ xii I4I.

I908 Chromatin unnecessary for cell division. AE xxvi 662.

I909a Conduction of excitation in ameba. "Mechanical hand." JEZ vi 85 (Plate I).

Igogb Nucleus is such as may act as statocyst in geotropism of paramecium. JEZ vi 87 (Plate II).

I909c Resistance of cell structure to centrifugal force and separation of phases of different density. AE xxvii 254 (Plate VI).

Igogd Physical differences between cells. AJP xxiii 460 .

Igoge The association of lecithin with protein in the frog's egg. AJP $\mathrm{XXV}$ I95.

I $909 \mathrm{f}$ Artificial parthenogenesis produced in a variety of ways. S $\mathrm{xxx} 454$.

I9ioa Frog's egg composed of 4 or more phases. AZ v 385.

IgIob Protoplasm is anodic and mitotic figure comparatively rigid. AE xxxi 80.

IgIoc Fertilization increases the conductivity of the egg. S xxxii I22. Igrod Fertilization decreases the sensitiveness of the egg to electrolysis and increases its sensitiveness to hypertonic sugar solution. $S$ xxxii 3 I 7 .

I9Ioe Fertilization or artificial stimuli increases the permeability of the egg. AJP xxvi 240.

I9I Ia Fertilization membrane. S xxxiii 387.

I9I Ib Electric shock causes frog's egg to segment. S xxxiii 629.

I9IIC Ameboid motion and permeability. AGP cxl $27 \mathrm{I}$.

rgI2a Pure $\mathrm{NaCl}$ solution increases permeability of Fundulus eggs.

Egg membrane or shell is freely permeable. AJP xxix 289.

I9I2b Artificial parthenogenesis of frogs and toads. AJP xxix 208. I9I2c Increased conductivity of tetanized muscles. AJP xxix 302. I9I2d Osmosis and surface tension in physiology. BB xxii II3.

I9I2e Alkaloids increase permeability of eggs. AJP xxxi I3I.

I9I2f Echinochrome is not a respiratory pigment. JBC xi 435 .

I9I3a. Increase in permeability of Fundulus eggs to salts. $\mathrm{S}$ xxxviii 280 .

I9I3b Surface tension and cell division. AE xxxvii 233.

Igr4a Increase in permeability of frog's egg. S xl 70.

I9I4b Antagonism of salts and anesthetics. $S \times 1$ 2r4.

I9I4C Permeability-increase of Fundulus eggs to $\mathrm{Cl}^{\prime}, \mathrm{Na}, \mathrm{K}, \mathrm{Ca}^{*}{ }^{*}$ and $\mathrm{Mg}^{\circ}$. IZPB i 28.

I9r4d Electric charge of protoplasm. IZPB i 159.

I9I4e Fertilization membrane. IZPB i I63.

I9I4f Osmotic swelling of frogs. IZPB i I69.

I9I4g Permeability of cells. CIWP (no. I83) I23.

I9I5a Fcrtilization or electric stimulus increases permeability of frog's egg to $\mathrm{Na}, \mathrm{K}, \mathrm{Mg}, \mathrm{Ca}, \mathrm{Cl}$ and $\mathrm{SO}_{4 *} \mathrm{AJP}$ xxxviii 163 . 
I9I5b Pure $\mathrm{NaCl}$ solution increases permeability of fish eggs to salts, and anesthetics inhibit this change. AJP xxxviii I73.

I9I5C Hydrogen electrodes. AJP xxxviii I80.

I9I5d Potentiometer for hydrogen electrode. AJP xxxviii I86.

I9I5e PH of stomach and duodenum of adult and infant. AJP xxxviii I9r.

$1915 \mathrm{f}$ Peptic digestion occurs in infant's duodenum. JAMA $1 \mathrm{xv}$ I2. I9I5g Pure oxyhemoglobin oxidizes a-napthol, aloin or p-phenylene diamine. JBC xxi 275.

I9I6a Hydrogen electrode and tonometer and conversion table. JBC xxiv 519 .

Igr6b Colored indicator chart. MRR xxii 333.

\& Magoon I9I6 Combined tonometer and hydrogen electrode. JBC $\mathrm{xxv} 669$.

\& Mitchell I9I2 Parth, and incr, oxidation of egg caused by $\mathrm{OH}^{\prime}$. JBC $\times 459$.

McClintic I9I2 Phenol coefficient of disinfectants. BHL (No. 82).

McCoy 1903 Dissociation of $\mathrm{CO}_{2 .}$ ACJ xxix 437.

MacDonald I905 Microanalysis of chlorides. PRS (B) 1xxvi 272, 322.

McDougail I898 Theory of muscle contractions. JAP xxxi, xxxii I93.

I908 Muscle contraction. JAP xxxii 193.

McGuigan 1904 Solution tension and enzyme action. AJP $\mathrm{x} 444$.

MacInnes I9I4 Transference no. and diffusion potential, JACS xxxvi 878 , 2301 .

McIntosh I897 Lyotrope series. JPC i 473.

Mackenzie I910 Gravitation. Bull. Minnesota Acad. Sc. iv 385.

McPhedran I9I3 Hemolysis. JEM xviii 527.

Magnus I900 Capillaries permeable to proteins. AEP xliv 68.

Igor Blood volume restored after increase by transfusion. AEP xlv 2 Io.

—, Sorgdrager \& Leeuwen I9I4 Lungs impermeable to $\mathrm{NH}_{3}$. AGP clv 275 .

Manape \& Matula 19r3 Protein binds little $\mathrm{Cl}^{\prime}$ of $\mathrm{NaCl}$. BZ lii 369 .

Mansfield 1908 Obesity and rate of resorption and circulation in narcosis. AIPd xvii 347.

Ig09 Narcosis and $\mathrm{O}_{2}$ solubility. AGP cxxix 69.

Marc I9II-3 Adsorption-saturation. ZPkC lxxvi 58; 1xxxi 64t.

I9I3 Adsorption and solid solution. ZPkC 1xxxi 64I.

Marriott $1916 \mathrm{CO}_{2}$ tension with indicator. JAMA lxvi 1594.

Masel I9r3 Acidosis in diabetic coma. ZKM lxxix I.

Masing I912 Permeability of erythrocytes to sugar, AGP cxlix 22\%.

1914 Temperature coefficient of diffusion of sugar into erythrocytes. AGP clvi 401 .

Massart 1889 Turgor regulation. $\mathrm{AB}$ ix 515 .

Mast IgII "Light and the Behavior of Organisms." N. Y. Wiley.

I9I4 Tropisms. BC xxxiv 64I.

\& Root I916 Surface tension and phagocytosis. PNAS ii r88; JEZ xxi I.

Mathews, A. 1902a Nerves lose excitability in non-electrolytes and regain it in salt solutions. S xV 492.

Igozb Current of injury. AJP viii 294.

I904a Solution tension and toxicity. Fundulus egg impermeable to $\mathrm{NaCl}$. AJP $\times 290$.

I904b Oxidising anions act best in acid and cations in alkali. AJP xi 237.

I904c Osmotic stimulation and solution tension. Ba stimulates nerve. AJP xi 455 .

1905a Electrolytic solution tension and toxicity. AJP xii 4 Ig. 
I905b Solution tension and precipitation of sols. AJP xiv 203. 1907a Toxicity of $\mathrm{NH}_{4}$ salts due to hydrolysis. AJP xviii 58 . I907b Cell division. AJP xviii 89. Ig0g Oxidation of sugar. JBC vi 3 .

I9I4 Narcosis and residual valence. IZPB i 433.

I9I5 Physiological Chemistry. N. Y. Wood.

\& Longfellow I910 Chemical theory of permeability. JPET ii 201 . Mathews, S. \& Brooks I9I0 Action of $\mathrm{MgSO}_{4}$. JPET ii 88 .

Matthews, D. I9I6 P in sea water. JMBA xi I22.

Mathison 19I I $\mathrm{H}^{*}$ stimulates vasomotor center. JP xlii 283 ; xli $4 \mathrm{I} 6$.

I9I I Effect of $\mathrm{PH}$ on reduction of arterial blood. JP xliii 5 .

Maxwell I905 Effect of salts on cilia. AJP xiii I54.

Mayenburg rooI Turgor regulation. JWB xxxvi 38I.

Mayer, A. G. Ig06 Pulsations of medusa (Cassiopea). CIWP (no, 47). I9I4a Electrolytes and rate of nerve conduction. CIWP (No. 183) 25. I9I4b Effect of temperature on animals. CIWP (No. 183) I.

I9I5 Nerve conduction. PNA i 270 .

I9I6 Nerve conduction. AJP xxxix 375.

Mayer, A. d. Ig02 "Gärungschemie."

Mayerhofer \& Pribram I909 Diffusion of colloids. ZEP vii.

\& Stein I9I0 Sugar increases permeability of gut. BZ xxvii 376 .

Means, Newburgh \& Porter I9I5 Increased Alv, $\mathrm{CO}_{2}$ in infections. clxxiii 742.

Meek \& Eyster I9I2 Positive variation of auricle after vagus stimulation. AJP $\mathrm{xxx} 27 \mathrm{I}$.

Meigs I908a Theory of muscle contraction. AJP xxii 477.

I908b Structure of muscle. ZAP viii $8 \mathrm{r}$.

I909a Heat rigor of muscle. AJP xxiv I, I78.

rgogb Water rigor. JP xxxix 384 .

I9I0 Swelling, and contraction of muscle. AJP xxvi I9r.

$1912 a$ Smooth muscle. QJEP v 55.

I9I2b Structure of smooth muscle. AJP xxix 317.

I9I2C Smooth muscle not semipermeable. JEZ xiii 497.

IgI2d Muscle permeable to $\mathrm{KCl}$. JEZ xiii 520 .

r9r4a Clam adductor. JBC xvii 8r.

I9I4b Swelling of muscle. AGP clviii $r$.

I9I5a Phosphate membranes. AJP xxxviii 456.

I9I5b Ash of clam muscle. JBC xxii 493 .

\& Atwood I9I6 $\mathrm{KCl}$ selectively absorbed by muscle. AJP xl 30.

- \& Ryan I9I2 Ash of smooth muscle. JBC xi $40 I$.

Mellor I909 "Higher Mathematics for Students of Chemistry, etc." 3rd Ed. Longmans.

Meltzer \& Auer 1905 Anesthesia with Mg. AJP xiv 36r. I $908 \mathrm{Ca}$ vs. Mg. ZP xxi 788.

Mendel, J. Gregor I865 Mendel's Law. VNVB iv.

Mendel, L. \& Thatcher I903 Secretion increases lymph of pancreas. AJP ix (p. xv).

Mendelssohn I916 Leucocytes are pos. galvanotropic. CRA 1 xii 52.

Menten I9I5 PH of stomach. JBC xxii $39 \mathrm{I}$.

- \& Crile I9I5 PH of blood. AJP xxxviii 225.

Menz 1908 Submicrons in gelatine. ZPC 1xvi I29.

Merrill I9I5 Exosnose from roots. AMBG ii 507.

Metcalf I905 Haptogen membranes. ZPC lii I.

Meyer, H. I899 Theory of narcosis. AEP xlii rog.

IgoI Narcosis and partition coefficient. AEP xlvi 338.

1909 Anesthetics increase permeability and cause mixing inside cells. MMW lvi 1577.

Meyer, K. IgII Bacterial protease. BZ xxxii 274. 
Meyer, L. \& Cohn I9II Water retention favored by $\mathrm{Na}$ and inhibited by Ca. ZK ii 360 .

Meyerhof IgIra Oxidation of fertilized egg greater in pure $\mathrm{NaCl}, \mathrm{BZ}$ xxxiii 29r.

19I Ib Thermodynamics of eggs. BZ Xxxv I59-184.

19r2a Thermodynamics of cells. AGP cxlvi I59.

I9I2b Reduction of dyes by dead cells. AGP cxlix 250 .

r9I4a Urethane retards oxidation of fertilized more than unfertilized eggs. Anesthetics do not lower osmotic pressure of proteins. AGP clvii $25 \mathrm{I}$.

19r $4 \mathrm{~b} \mathrm{H}_{2} \mathrm{O}_{2}$ catalysis by $\mathrm{Pt}$. inhibited by anesthetics. AGP clvii $30 \%$. I9I5 Anesthetics and enzymes. JZPB ii 394.

Michaelis I908 Adsorption of colloids irreversible. BZ xii 26.

1908 Solution tension and polarization. ZEC xiv 353.

I909 Calculation of isoelectric point. BZ xix I8I.

I912 Isoelectric point of proteins. Nernst-Festschrift. 308.

I9I4 "Die Wasserstoff ionenkonzentration." Berlin.

\& Davidsohn I9ro Indicators for stomach. ZEP viii 398.

rgro Isoelectric point. $\mathrm{BZ} \times \mathrm{xxx}$ I43.

I9II Optimum PH for invertase. BZ xxxv 386.

I9I3 Gelatine stains with acid dyes in acid solution. BZ liv 323.

- \& Ehrenreich I908 Acid dyes and invertase adsorbed by clay but not by kaolin. BZ $\times 283$.

- \& Menten I9I3 Action of invertase inhibited by fructose. BZ xlix 333.

\& Mostynski I9Io Viscosity and ionization of proteins. BZ xxv 401. \& Perkins I9I4 "Dynamics of Surfaces." London.

\& Pincussohn I906 Ultramicroscopy of colloid reactions. BZ ii $25 \mathrm{I}$.

\& Rona I907 Protein precipitated by dialysed iron. BZ ii, iii, iv, v. Ig08a Osmotic compensation. BZ xiv 476.

I908b Adsorption of colloids irreversible. Competitive adsorption. $\mathrm{BZ} \mathrm{xv}$ 196.

Ig09a Competitive adsorption. BZ xvi 489.

Igogb Effect of salts on Congo red. BZ xxiii 6I.

I910 Isoelectric point of denatured albumin. $\mathrm{BZ}$ xxvii 38 .

I9I2 Isoelectric points of emulsions. BZ xxxix 48I.

_ \& Takehashi I9Io Hemolysis and isoelectric point. BZ xxix 439.

Miculicich 19I0 Effect of ions on hemolysis by anesthetics. ZP Xxiv 523 .

Millikan r9ro Charge of an ion. PM xix 209.

I9I6 Equation for contact electricity. PR vii I8.

Milroy I9I4 PH and respiratory center. QJEP viii I4T.

Mines rgrra $\mathrm{Ca}, \mathrm{Mg}, \mathrm{Sr}$ and $\mathrm{Ba}$ inhibit the stimulating action of $\mathrm{K}$ on muscle, JP xlii $25 \mathrm{I}$.

I9IIb Ppt. of $\mathrm{As}_{2} \mathrm{~S}_{3}$ by cations. Action of rare earth salts on emulsoids and reversal of emf of concentration cell with gelatine diaphragm. JP xlii 309 .

I9IIc PH of pecten blood $=6.7$. Heart stops in diastole in acid, systole in alkali. JMBA ix I7r.

I9I2 $\mathrm{PH}$ for heart of pecten. JP xliii 467.

I9I3a Action of ions. JP xlvi I88.

I9I3b Dynamic equilibrium of the heart. JP xlvi 349.

Milner I907 Adsorption and surface tension. PM (6) xiii 96.

Mitscherlich 1893 Ignition point of $\mathrm{H}_{2}+\mathrm{O}_{2}$ raised by pressure, BDCG xxvi 399 .

Moldovan \& Weinfurter I904 Anesthetics reduce cell respiration. AGP clvii 57 I.

Momose I9I5 Alv. $\mathrm{CO}_{2}$ and $\mathrm{PH}$ of blood. BJ ix 485 . 
Montgomery 1878 St. Thos. Hos. Repts. London, n, s, ix.

I88I Muscle contraction and ameboid motion. AGP xxv 497.

Mond, Ramsay \& Shields 1898 Absorption of $\mathrm{H}_{2}$ by metals, PRS Ixii 290 .

Moore, A. Igroa Temp. coef. of cytolysis and hemolysis $=200$ for $10^{\circ}$. QJEP iii 257.

I9Iob Temp. coef. of regeneration. AE xxix I45.

Moore, B. I9I4 Iron and photosynthesis, PRS (B) lxxxvii 556.

- \& Roaf I 906 Lipoids and proteins precipitated by anesthetics in low concentration. PRS (B) lxxvii 86.

I907 Osmotic pressure of colloids. BJ ii 34 .

I 908 More salts dialyze from serum than from erythrocytes. BJ iii 55 .

- \& Webster I9I3 Photosynthesis of formaldehyde from $\mathrm{CO}_{2}$ and $\mathrm{H}_{2} \mathrm{O}$. PRS (B) 1xxxvii I63.

\& Whitley 1909 Oxidases. BJ iv 136.

Morawitz, $\mathrm{H}$. I9ro Adsorption of $\mathrm{HgCl}_{2}$ by erythrocytes. $\mathrm{KZ}$ vi 259 .

IgIo Adsorption of antiseptics by bacteria. Reduction of heavy metals by adsorption. $\mathrm{KCB}$ i $30 \mathrm{I}$.

Morawitz, P. I909 Oxidation in blood. AEP 1x 298.

Morgulis \& Fuller $\mathrm{CO}_{2}$ in sea cannot be titrated with phenolphthalein. JBC xxiv $3 I$.

Morgan, J. IgI5 Measurement of surface tension. ZPkC.

Morgan, T. \& Stockard Igo7 Effects of salts and sugar on frog's egg. $\mathrm{BB}$ xiii 272.

Morse, H. I914 Osmotic pressure of sugar solutions. CIWP (No. I98).

\& Horn I90I Osmotic membranes. ACJ xxvi 80.

Morse, M. I9ra Eggs in sperm extracts. JEZ xiii $47 \mathrm{I}$.

IgIab Parthenogenesis. JEZ xiv $47 \mathrm{I}$.

I9I5 Halogens and enzymes. JBC xxii I25.

1916 Autolysis is autocatalyzed by $\mathrm{H}^{\circ}$. JBC xxiv 163.

Moruzzi Igro Urea liberates $\mathrm{NH}_{8}$. $\mathrm{BZ}$ xxviii 97.

Mourson \& Schlagdenhauffen I882 Sea urchin blood. CRA xcv 79 r.

Mouton 1897 Turgor regulation. CRA cxxv 407.

Müller, C. IgI2 Absorption of gases by non-electrolyte solutions, ZPkC $1 \times x \times 1483$.

Müller-Thurgau I880 Capillarity and undercooling. LJ ix I76. I 886 Undercooling of plants. LJ xv 490.

Murlin, Edelmann \& Kramer $\mathrm{I} \mathrm{I} 3 \mathrm{CO}_{2}$ and $\mathrm{O}_{2}$ of blood in stasis. JBC xvi 79.

\& Kramer I9r3 Respiration in glycosuria. JBC xv 365.

Myers \& Acree I9I3 $\mathrm{H}_{2}$ electrode. ACJ 1396.

Nägeli I855 "Pflanzenphysiologische Untersuchungen." I885 "Pflanzenphysiologische Studien." Zurich.

Nagel Ig09 "Handbuch der Physiologie des Menschen." Braunschweig. F. Vieweg.

Nasse 1869 Swelling of muscle. AGP ii 97.

Nathansohn 1904 Lecithin changes permeability when wet. JWB xxxix 607.

Nemec 1900 Geotropism of plants. BDBG xviii 241 .

Neilson Igo6 Platinum black poisoned by $\mathrm{HCN}$ in splitting of amygdalin. AJP $\mathrm{xv}$ I48.

Nernst I888 Formula for electrode and diffusion potential. ZPkC ii $6 \mathbf{3}$, 639.

I889 Formula for diffusion potential. ZPkC iv 129.

I890 Permeability and solution in plasma membrane. $Z P k C$ vi 37.

I892 Solution tension and polarization. $\mathrm{ZPkC}$ ix I37.

I897 Tesla currents do not stimulate. APk lx 600.

IgII "Theoretical Chemistry." Trans. of 6th Ed. Macmillan.

— \& Barratt I904 Verification of Nernst's theory of stimulation. ZEC 663. 
\& Riesenfeld I902 Diphasic concentration cells. APk (4) viii 600. Neuberg r9II "Die Harn sowie die uebrigen Körperflussigkeiten." Berlin. Neufeld \& Haendel - I908 Hemolysins are cytolytic. Bile salts dissolve blood shadows. AKG xxviii 572.

Nicloux 19I3-I4 Absorption of $\mathrm{CO}_{2}$ by blood. CRA clvii 1425, clviii 363 . Nishi I9I0 Sugar is secreted by glomeruli and resorbed by tubules. AEP lxii 329.

Noguchi I902 Combination of lecithin and saponine. UPMB (Nov.). 1905 Snake venom protects erythrocytes. JEM vii I9I.

Nolf I904 $\Delta$ of venous and arterial blood. AB xx I.

Norton \& Hsu I9I6 Disinfection. JID xviii 180.

Nothmann-Zucherkandl 19I2 Protoplasmic streaming quickly stopped with anesthetics but continues weeks without $\mathrm{O}_{2}$. $\quad \mathrm{BZ}$ xlv $4 \mathrm{I} 2$.

I9I5 Toxicity of anesthetics increased by salts. JZPB ii 19.

Novak, Leimdoerfer \& Porges 1902 Alveolar $\mathrm{CO}_{2}$ during pregnancy. ZKM 1xxy 30I.

Noyes 1907 Electric conductivity. CIWP (No. 63).

- \& Blanchard 1900 Color of Co ions and molecules. JACS xxii 726.

- Melcher, Cooper \& Eastman I9Io Conductivity at high temperatures. $\mathrm{ZPkC} 1 \mathrm{xx} 335$.

Okada Igr 5 Liver bile $\mathrm{PH}=7.75$, gall more acid. JP 1 II4.

Oker-Blom Igoo Conductivity of sand $+40 \%$ solution $=25 \%$ of conductivity of solution. AGP lxxix 5 I0.

I90I Bioelectric currents. AGP 1xxxiv IgI.

Onaka I9I I Oxidation of blood platelets. ZPC 1xxi 193.

Oppenheimer Igog Handbuch der Biochemie des Menschen und fler Tiere. I913 "Die Fermente." Leipzig.

Osborne Igo6 Intracellular colloid salts. JP xxxiv I27.

Osterhout I9Io Permeability of cells to Ca. ZPkC 1xx 408.

I9II Permeability of cells, S xxxiv I87.

1912a Permeability. S xxxv II2.

$1912 \mathrm{~b}$ Plants requiring $\mathrm{Na}$. BG liv 532.

I9I2c Change in permeability. S xxxvi 350 .

I9I3a Pseudo plasmolysis. BG lv 446.

1913b Anesthetics and permeability. S xxxvii IIx.

I9I4a Alkali and permeability. JBC xix 335.

I9I4b Acid and permeability. JBC xix 493.

19r5a Acids vs. salts. JBC xix 5 I7.

19I5b Polyvalent ions and permeability. BG lix 464.

I9I5c Permeability. AJB ii 93.

I9I5d Decrease in perm. BG lix 317.

I9I5e Abnormal permeability without death. BG 11x 242.

Ostwald, Wm. I889 Dilution law. ZPkC iii I70.

I8go Elec. properties of semipermeable membranes. Becquerel phenomenon. $\mathrm{ZPkC}$ vi $7 \mathrm{I}, 74$.

I892 Color of ions. $\mathrm{ZPkC}$ ix 579.

I897 Metastable state. ZPkC xxii 302.

I899 "Grundriss d. allg. Chemie." 3rd Ed. Leipsic.

I900 Small crystals more soluble than large. ZPkC xxxiv 495.

\& Luther Igro "Handbuch Physiko-Chemischer Messungen." 3rd Ed. Leipsic.

Ostwald, Wo. 1905 Gammarus pulex will live in fresh or sea water. AGP cvi 568.

1907a Disperse systems. $\mathrm{KZ}$ i $29 \mathrm{r}$.

I907b Adsorption and toxicity. AGP cxx I9.

1907c Oxidases sensitive to light. $\mathrm{ZB} \times \mathrm{r} 30$.

1908 Dissociation of oxyhemaglobin. $\mathrm{KZ}$ ii 264 .

I909 Grundriss der Kolloidchemie. Dresden, Steinkopff. 
I912 Color of indicators. Dispersion of dyes. $\mathrm{KZ} \times 97$.

I 913 Viscosity of sols. TFS ix 34.

Otto I9I3 Narcosis and evolution. ZB lix I65.

Overton I 895 Plasmolysis and permeability. VNGZ xl r.

I 897 Cells permeable to free alkaloids but not their salts. Permea-

bility to $\mathrm{NH}_{3}$. ZPV xxii 189 .

I899 Permeability of cells. VNGZ xliv 88.

1900 Permeability and partition coefficient. JWB xxxiv 669.

I90I "Studien ueber die Narkose." Jena.

I902a Permeability of muscle $0.65 \% \mathrm{NaCl}$ for frog. AGP xcii II5. $1902 \mathrm{~b} \mathrm{Na}$ (or Li) necessary for muscle contraction. AGP xcii 346 . I904a Frog's kidneys regulate osmotic pressure. VPMG (NF) xxxvi 277.

I904b Ions restoring excitability of sugar muscle. AGP cv $I 76$.

I907 Frog's egg membrane permeable to crystalloids. II p. 878 . Nagel's Handb. d. Physiol. d. Menschen.

Packard 1905 Injection of alkali decreases symptoms of $\mathrm{O}_{z}$ want. AJP $\mathrm{xv} 30$.

Paine 1912a Permeability of yeast. PRS (B) lxxxiv 289.

I9I2b Decrease in dispersion of sols and rate of precipitation by electrolysis. $\mathrm{KCB}$ iv 24 .

Palitzsch I9Ira PH by methyl red. BZ xxxvii I3I.

I9I Ib $\mathrm{PH}$ of sea water. $\mathrm{BZ} \times \mathrm{x} \times \mathrm{x}$ ii II6.

I9I5 Boric-borax mixtures for PH. BZ $1 \mathrm{xx} 333$.

Pantanelli I90.4 Osmotic pressure of mould on concentrated $\mathrm{KNO}_{3}$. Colloid swelling and turgor. JWB xl 303.

I 906 Acetates increase permeability of fungi to enzymes. AB v 355.

Pascheles I898 Coagulation in emulsoid gels. AGP 1xxi 333.

Pascucci 1905 Erythrocyte stroma $1 / 3$ lipoid and $2 / 3$ proteins. Artificial lipoid membranes. BCPP vi 543.

I907 Perm. of lipoid mem. to pro-enzymes. BZ vi 358.

Pasteur I858 Asymmetric fermentation of tartaric acid, CRA xlvi 6I5.

Paul I9OI Ionization hastens toxic action of heavy metals. ZPkC xxxvii 754.

Birstein \& Reuss I9ro Adsorption of antiseptics by bacteria. BZ xxix 202.

\& Krönig 1896 Disinfection with $\mathrm{Hg}$ ions. $\mathrm{ZPkC}$ xxi $4 \mathrm{I} 4$.

Pauli I903 Lyotrope series for alkali albumin reversed for acid albumins.

$\mathrm{Ba}, \mathrm{Sr}$ and $\mathrm{Ca}$ precipitate proteins irreversibility. BCPP v 27.

I905 Precipitation of emulsoids with heavy metals. BCPP vi 233.

I 906 Electric charge of protein. BCPP vii 53 I.

I907 Heat coagulation of acid albumin. BCPP $\times 53$.

Igro Colloidal state and physiology. AGP cxxxvi 483.

I9I2 "Kolloidchemie der Muskelkontraktion." Dresden. Steinkopff.

\& Falek I9I2 Caffeine and hydration of proteins. BZ xlvii 269.

\& Flecher I912 Precipitation of emulsoids with suspensoids. BZ x.1i $46 \mathrm{r}$.

\& Handovsky 1908 Ion-protein compounds. BCPP xl 4I5.

Ig09 Acid albumin. BZ xviii 340 .

1910 Alkali-albumin. BZ xxiv 239.

\& Matula I9I6 Thermo-current of muscle. AGP clxiii 355 .

\& Rona 1902 Swelling of gels. Glycerine and sugar favor and urea inhibits gelation. $\mathrm{BCPP}$ ii $\mathrm{I}$.

\& Samec I909' Proteins increase solubility of $\mathrm{Ca}_{3}\left(\mathrm{PO}_{4}\right)_{2}$ and $\mathrm{CaCO}_{3}$ but not of alkali salts appreciably. BZ xvii 235 .

I9I 3 Viscosity and optical rotation of emulsoids. KZ xii 222.

, Samec \& Grauss I914 Optical rotation of protein salts. BZ lix 470.

- \& Wagner I 1 ro Acids and alkalis increase viscosity of proteins. BZ xxvii 296. 
Pavloff 1910 "The Work of the Digestive Glands." Trans. of 2nd Ed. London.

Peabody I9I4 No relation of $\mathrm{PH}$ to dyspnoea in cardio-renal. AIM xiv 236. I9I6 Alv. $\mathrm{CO}_{2}=6 \mathrm{~mm}$ in diabetic coma. AJMS cli 184 .

Pearl \& Gowan I9I4 Refractive index of serum. PSEB xii 48.

Pekelharing I9I4 Nucleo-protein, Ca and blood coagulation. ZPC 1xxxix 22.

\& Ringer I9II Cataphoresis of pepsin. ZPC 1xxv 282.

Pelet-Jolivet \& Anderson rgo8 Adsorption and electric charge. KZ ii 225.

Perrin 1904-5 Dielectric constant and contact electricity. Formula for cataphoresis. JCP ii 60r, iii 50.

Ig08 Stokes law and Brownian movement, CRA cxlvi 960, cxlvii 475 . I909 Ultramicroscopy of coagulation. ACP (8) xviii 89.

IgIl Avogadro's No. $=6 \times 10^{23}$ molecules in mol, wt. CRA clii $1 \mathrm{I} 65$, 1382.

Pfäundler I905 PH of blood of premature babies $=6.55$. Normal $=7.5$. AK xli i6r, I74.

Pffeffer I873 "Physiologische Untersuchungen." Leipsic.

I877 "Osmotische Untersuchungen." Leipsic, Engelmann.

- \& Hansteen 1893 Splitting of starch in germinating corn inhibited by sugar. BVGW xlv 42 r.

Pffeiffer \& Von Modelski I912-3 Amino acids bind NaC1. ZPC lxxxi $329,1 \times x V I$.

Philip IgI4 "Physical Chemistry and Its Bearing on Biology." and Ed. London.

Philips Igo7 Effect of solutes on solubility of gases. TFS iii I40.

Phillipson, Hannevart \& Thieren tgro $\Delta$ of fresh water animals. AJP ix 460 .

Philoche I908 Maltase action is autocatalytic. JCP vi 2 I3, 355.

Pickering I9I0 Haptogen membranes and emulsions. $K Z$ vii II.

Pictet 1893 Diatoms live at $-200^{\circ}$. ASPN xxx 3II.

Picton \& Linder 1892 Colloids show Tyndall phenomenon. JCS 1xi I48.

Pierce rgro Coagulation of substrate and enzyme action. JACS xxxii I5I7.

Piper I9I2 "Elektrophysiologie Menschlicher Muskeln." Berlin.

Planck 1890 Formula for diffusion potential. APC x1 56r.

Plesch I909 Hemodynamics. ZEP vi.

Poh1 I891 Chloroform is concentrated by erythrocytes, lecithin, cholesterin and oil. AEP xxviii 2 Io.

Polanyi I9II Blood is acid in starvation. BZ xxxiv I92.

Porges Ig05 Agglutinin or heat transforms bacteria into suspnesoids. $\mathrm{ZB} \times 1 \mathrm{I33}$.

I9I I Acidosis. WKW.

I9I3 $\mathrm{CO}_{2}$ tension and respiration. $\mathrm{BZ}$ liv $\mathrm{I} 82$.

\& Neubatuer I907 Lecithin precipitated by $\mathrm{Ba}, \mathrm{Sr}, \mathrm{Ca}$ and $\mathrm{Mg}$ like suspensoid. BZ vii I52.

Port rgro Effect of ions on hemolysis by saponin. Resistance greater the less $\mathrm{P}$ (lecithin?) erythrocytes contain. DAKM xcix 259.

Porter I9I I Is rennin pepsin? JP xlii 389.

I9r4 Adsorption of trypsin by serum alb. BJ viii 50.

Portier I9ro $\Delta$ of marine vertebrates. JPP xii 202.

Posnjak rgI2 Swelling pressure of gelatine. KCB iii $4 \mathrm{I} 7$.

Poulton IgI5 Alv. $\mathrm{CO}_{2}$ below $2.5 \%$ anticipates coma. PRS (M) vii I7I; JP 1 (p. i).

Pototsky Igo2 Diuresis and $\mathrm{NaCl}$ starvation. AGP xci 584.

Prenant ro10 Mitosis. JAP xlvi 51 I.

Pribram IgII Lipoids precipitated by anesthetics. AGP cxxxvii 350.

Price IgI4 Ultramicroscopy of plant cells. AB xxviii 60I.

Prideaux IgI I PH of phosphate mixtures. BJ vi I22. 
Pringsheim 19I2 "Die Reizbewegungen der Pflanzer."

Prowazek 1903 Phagocytosis of echinoderm egg. ZAP ii 385.

I907 Formation of plasma membrane. BC xxvii 734 .

Pugliese 1906 Effect of salts on smooth muscle. AIB xivi 371 .

Putter 1904 Toxicity of great $\mathrm{O}_{2}$ pressure. ZAP iii 363. Igr I Vergleichende Physiologie. Jena, Fischer.

Pulst $1902 \mathrm{Cu}$ non toxic to penicillium. JWB xxxvii 205.

Quagliariello I9ro Saline cathartics. BZ xxvii 517. I9I2a Change of $\mathrm{PH}$ during heat coagulation of proteins. BZ xliv 157. I9ı2b $\mathrm{PH}$ normal in fever. BZ xliv I62.

Quincke I888a Plasma membrane fat. APk xxxv 580; SKPA xxxiv 791. I888b Adsorption of colloids. APk xxxv 582. I902 Surface tension of sols. APk ix 969.

Quinton I900 $\Delta$ of marine animals. CRA cxxxi 952. I904 Change of $\Delta$ of fish. CRB lvii 470.

Raben I905 $\mathrm{N}$ and $\mathrm{Si}$ in sea water. Wissensch. Meeresunts. Kiel, viii $8 \mathrm{I}-286$.

Radziszewski 1883 Compounds that emit light on oxidation at low temperature. BDCG x 597.

Raehlmann I906 Ultramicroscopy of protein. AGP cxii I28.

Ramsay I894 Diffusion pressure of gases. PM xxxviii $206 ; 2 \mathrm{PkC}$ xv 5 I8.

Ramsden I904 Haptogen membranes. PRS 1xxii I56; ZPkC xlvii 343.

Ransom IgOI Cholesterin protects erythrocytes from saponin. DMW I94.

Raoult Igor Formula for osmotic pressure. AUG xiii I73.

Raulin I870 Salt solution for plants. CRA $1 \times x 634$.

Rayleigh 1879 Surface tension measurements. PRS xxix 71. I899 Tyndall phenomenon and color of slky. PM xlvii 375.

Rayleigh, G. B. I9I5 Oxidasis and respiration. JBC xxii 99.

Reid I900 Ligature of lymphatics does not decrease absorption by gut. PT (B) cxcii 23I.

I90I Negative osmose of diaphragm of live gut wall. JP xxvi 436. I904 Osmotic pressure of purified protein $=0$. JP xxxi 438 .

Rehfuss I9I5 Achylia gastrica. AJMS cl 72.

-, Bergein \& Hawk I9I4 Residuum in empty stomach. JAMA 1xiii II.

— \& Hawk I9I4 Constant acidity of gastric juice. JAMA 1xiii 2088.

Reicher Ig08 Anesthesia leads to pathological acid production. ZKM v 235.

Reinders I9I3 Abnormal partition coefficient and hydrolysis. KZ xiii 96.

Rhumbler 1898 Contractile vacuole. AE vii 103. 1905 Ameboid motion. ZWZ Ixxxiii $x$.

I9I0 Phagocytosis. AE xxx I94.

Ribbert I9I3 Path of excretion of hemoglobin by kidney. ZP xxiv 244.

Richards, A. IgI4a X-rays and cell division. BB xxvii. I9I4b X-radiation of enzymes. AJP Xxxy 224. I9I5a X-radiation. AJP xxxvi 400.

I9I5b Physiology of radioactivity. S xlii 287.

Richards, T. I897 n/ro calomel electrode. ZPkC xxiv 39. 1906 Nephelometer. ACJ xxxv 510.

I9I5 Atomic compressibility. JACS xxxvii I643.

\& Wells I904 Nephelometer. ACJ.

Richardson, Nicol \& Parnell Ig04 Diffusion of $\mathrm{H}_{3}$ (as $\mathrm{H}$ ) through Pt. PM (6) viii I.

Riddle \& Spohn I9I2 Change in composition and permeability. S xxxv 462. Rigg I9I6 Salts in kelp. S xliii 602.

Ringer, S. I880-3 Effect of salts on heart. JP iii 380, iv 29, 222. I886 Effect of salts on muscle. JP vii 29 I.

\& Phear I895 Effect of salts on tadpoles. JP xvii 423.

- \& Gainsbury 1890 Salts and coagulation of blood. JP xi 369.

Ringer, W. Igro Uric acid and phosphates. ZPC lxvii 332. 
I916 Pepsin anodic even in acid. $\mathrm{H}^{\circ}$ acts on substrate. VKAW xviii 738 .

\& Van Trigt I9I2 PH and ptyalin. ZPC 1xxxii 484.

Roäf I9I0 Osmotic pressure of proteins reduced by salts. QJEP iii 75, I7I. I9I2a Hemolysis. QJEP v I3I.

19ז2b Marine organisms. JP xliii 449.

$1913 \mathrm{O}_{2}$ tension of tissues. PRS (B) 1xxxvi 215.

I9I4 Muscle contraction. PRS (b) 1xxxviii I39.

Robertson Igo6 Strychnine antitoxic to $\mathrm{Na}_{2} \mathrm{SO}_{4}$ J JC i 507.

1907 Dissociation of proteins. JPC xi $437,542$.

I9IO Dissociation of acid and alkali albumins. JPC xiv 377, 528 .

I9II Cell division. AE xxxii 308.

rgז2 Oocytin from serum and sperm. JBC xii.

Rodier $1899 \Delta$ of marine vertebrates. TLA I03.

Rohde 1906 Haptogen membranes. AP (4) xix I35.

Rohonyi I9I2 Protein binds $\mathrm{H}^{\circ}$ and $\mathrm{Cl}^{\prime},{ }^{\mathrm{BZ}}$ xliv $\mathrm{I} 65$.

Rolly I9I2 PH in disease. MMW.

I9I3 PH in disease. $\mathrm{ZN}$ xlvii $6 \mathrm{I} 7$.

Rona I901 $\mathrm{Cl}^{\prime}$ in blood. $\mathrm{BZ}$ xxix 501 .

\& Doeblin I910 Permeability of erythrocytes to sugar. BZ xxxi 2I5.

- \& Gyoergy 1913 Diffusible $\mathrm{Na}$ and $\mathrm{CO}_{2}$ in blood. $\mathrm{BZ}$ xlviii 278 .

I9r 3 When $\mathrm{PH}$ is less than 4.8 serum protein binds $\mathrm{Cl}^{\prime}, \mathrm{CO}_{2}$ increases diffusible alkali in serum. $\mathrm{Na}, \mathrm{K}^{\prime}, \mathrm{Cl}^{\prime}$ and free $\mathrm{CO}_{2}$ in blood. BZ lvi $4 \mathbf{1} 6$.

\& Michaelis Igo9a Charcoal adsorbs sugar. BZ xvi 489.

Igogb Permeability of erythrocytes. BZ xvi 6o, xviii 375 .

Ig0oc $\mathrm{Ca}^{\prime \prime}$ in milk. BZ xxi II4.

__ \& Wilenko I9I4 PH and use of sugar by heart. BZ lix I73.

— \& Takahashi I9Io Permeability of erythrocytes to sugar. BZ xxx 99. IgII $\mathrm{Ca}^{\prime \prime}$ in blood. BZ xxxi 336 .

I913 All $\mathrm{Na}^{\circ}$ and $\mathrm{K}^{\circ}$ in serum are diffusible. $1 / 3 \mathrm{Ca}$ is bound. $\mathrm{BZ}$ xlix 370.

_- \& Tosh 1914 Urethane displaces adsorbed dextrose from animal charcoal. BZ lxiv 288 .

Röntgen \& Schneider 1886 Adsorption of ions. Negative adsorption. AP xxix 165 .

Rosa 1913 Weston cell $=1.01827$ at $20^{\circ}$. BBS ix 493.

Rosenthaler I910 Synthesis of amygdalin with emulsin. BZ xxviii 408.

Ross I9I I "Induced Cell Division and Cancer." Blakistons.

Rossi 1909 "La Reatione delle urine nelle forme febrille." Naples.

Rost I9I I Nucleus permeable to dyes. AGP cxxxvii 359.

Rowntree, Marriott \& Levy I9I5 PH of dialysate with indicator. JHHB Xxvi II4.

Rubner r913 Fermentation by yeast same in 2.5\% and in $20 \%$ sugar. Synthesis of glycogen. AAP (Sup.) 240 .

Rudolphi 1895 Dilution law for strong electrolytes. $\mathrm{ZPkC}$ xvii 385.

Ruhland I908 Diffusion through artificial lipoid membranes and permeability to acids. JWB xlvi I.

I9I2 Ultrafiltration and vital staining. BDBG $\times x \times \quad 139$.

r913 Colloids and cells. KZ xii II 3 .

Ryffel Ig09 Lactic acid and acidosis. JP xxxix (p. xxix).

Rywosch Ig07 Erythrocytes of ox and sheep containing most cholesterin are most resistant to saponine and least to hypotonicity. AGP cxvi 229.

Salamonson I9I6 Psychogalvanic reflex. VKAW x 997.

Salge I9II $\Delta$ of newborn. KZ i 126, ii 347 . I9I2 $\mathrm{PH}$ of newborn. KZ iv I7I.

Salm I906-8 Indicators. $\mathrm{ZPkC}$ lvii $47 \mathrm{r}$, $1 \times$ vii 83. 
Salzmann I913 Individual factors in narcosis, AEP 1 xx 233.

Samec Igr2 Swelling of starch: $\mathrm{Li}, \mathrm{Mg}, \mathrm{Ca}, \mathrm{Na}, \mathrm{K}, \mathrm{Sr}, \mathrm{Ba} . \mathrm{KCB}$ iii I, I23.

Savchenko \& Aristovsky I9I2 PH and phagocytosis. ASB xvii 128.

Scatchard \& Bagert I9I6 Dinitrobenzoylene-urea PH 6-8. S xliii 722.

Schade I9I0 Gall and bladder stones due to lack of protection colloids. KCB i 375 .

I9I0 Lyotrope series and association of $\mathrm{H}_{2} \mathrm{O}$ color of sols. $\mathrm{KZ}$ vii 26. I9I3 Tendons swell in acid and shrink in alkali. ZEP xiv $\mathbf{I}$.

- \& Boden I9I3 Colloidal solutions of uric acid. ZPC 1xxxiii 347; Ixxxvi 238.

Schafer I902 Swelling of frog's embryos. AE xiv 306.

Schaffnit IgI Freezing out of colloids ZAP xii 223.

Schaffer 1888 Rate of diffusion. ZC ii 390.

Scheurlen $1896 \mathrm{NaCl}$ increases toxicity of phenol. AEP xxxvii 84 .

\& Spiro 1897 NaCl increases toxicity of phenol. MMW 81.

Schimkewitsch I902 Amitosis. BC xxii 605.

Schlaepper tg06 Oxidation in cells. AGP cxiv 301.

Schloss 1912 Water retention favored by $\mathrm{Na}$ and inhibited by $\mathrm{Ca}$. $\mathrm{ZK}$ iii $44 \mathrm{I}$.

Schmidt, C. Ig09 Conversion table: millivolts and $\mathrm{H}$ : UCPP iii IoI.

Schmidt, G. IgIo Adsorption saturation. ZPkC 1xxiv 689, lxxvii 64I.

Schmidt-Neilsen 1909 Change of $\Delta$ of fish. NVS 20.

I909-Io Adsorption of colloids irreversible. ZPC 1xviii 3I7; 1x 426.

Schneider I9I3 Alveolar $\mathrm{CO}_{2}$ tension after descent. A]P xxxii 295.

Schönbien I863 Catalysis. JPC lxxxix 323.

Schorr I9I2 Protein most easily precipitated by albumin at isoelectric point. BZ xxxviii 424 .

Schryver I9I0 Photosynthesis of formaldehyde. PRS (B) 1xxxii 226.

Schucking 1903 Fertilization membrane preformed. AGP xcvii 85 .

Schütt I904 Haptogen membranes. APm (4) xiii 7 I2.

Schütz I885 Peptic digestion proportional to square root of pepsin concentration. $\mathrm{ZPC}$ ix 577 .

Schulemann I9I2 Dispersion and vital staining of Goldmann pyrol cells. APm ccl 252.

Schulz I9I2 $\Delta$ of newborn. ZK iii 25I, 405.

Schulze I884 Schulze's rule (valence). JPkC xxvii 320.

Schwarz I907 Ions restoring excitability of sugar muscle. AGP cxvii I6r. IgI T Tetanus increases osmotic pressure of muscle. BZ xxxvii 34.

\& Lemberger IgI I Effect of $\mathrm{H}^{\circ}$ on blood vessels. AGP cxli I49.

Schwartz I9I3 Skin current of frog. ZP xxvi 734. 1915 Frog's skin current. AGP clxii I62, 547.

Schücking I902 Marine invertebrates semipermeable. AAP 533.

Schwyzer Ig14 PH and blood corpuscles. BZ 1x 297, 306.

Scott, F. Ig08 Regulation of respiration. JP xxxvii 301.

I9I5 Permeability of erythrocytes to protein. JP 1 I28.

I9I6 Permeability of blood vessels. JP 1 I 57.

Scott, G. I9I3a Osmotic equilibrium of fish. BB xxy I2I. I9r3b Osmotic equilibrium of fish. ANYA xxiii I.

Scott, R. I9I6 PH and dissociation of oxyhemoglobin. JLCM i 608 .

Scudder I9I4 "Conductivity and Ionization Constant of Organic Compounds." N. Y.

Sellards I9I4 Acidosis in nephritis. JHHB xxv I4I.

Senter I903-Io Enzymes. ZPkC xliv 257, li 673, 68r, 701, lii 5II.

Shaklee \& Meltzer I909 Shaking enzymes. AJP xxv 8I.

Shattuck \& Dudgeon I9I2 Effect of drying bacteria. PRS (B) 1xxxv I27.

Sheppard I9I4 "Photochemistry," Longmans.

Shields 1893 Hydrolysis of KCN. ZPkC xii 167. 
Shorter I909 Haptogen membranes. PM (6) xvii 560.

\& Ellingworth I916 Soap and not $\mathrm{OH}^{\prime}$ emulsifies. PRS (A) xcii 23I. Shull Igr3 Permeability of hulls of Xanthium seeds like plasma membrane. BG lvi I69.

Siebeck I9I2 Permeability of kidney cells. AGP cxlviii 443. rgI3 Permeability of muscle to $\mathrm{KCl}$. AGP cl 316 .

Siedentopf \& Zsigmondy 1903 Ultramicroscope. APk x I.

Simon I9Io Protein-salt compounds. ZPC 1xvi 70.

Slator I903 Catalyser may alter type of reaction. ZPkC xlv 5 I3.

Snyder I908 Temp. coef. of vital processes. AJP xxii 309.

Sollman 1903 Selective action of kidney. AJP ix 425, 454. I904 Fundulus egg permeable to water (?). AJP xii II2.

- \& Pitcher I9I I Asphyxia stimulates vasomotor center. AJP xxix 100.

Sörensen, S. P. L. Ig09 $\mathrm{H}^{\circ}$ and enzymes. BZ xxi I30, xxii 352. I912 PH methods. EP xii 393.

\& Palitsch I9ro Alphanaptholphthalein: BZ xxiv 381. I913 PH of sea water. BZ li 307.

Souza I9I I Reduction of thermolability of trypsin. JP xliii 374.

Spaeth I9I3 Effect of salts on chromotaphores. JEZ xv $52 \%$.

Spiro I904 Acids and alkalis increase swelling of gelatine. BCPP v 276.

— \& Bruns $1898 \mathrm{NaCl}$ increases toxicity of phenol. AEPP xli 355 .

— \& Henderson, L. I908 Model of blood alkalescence. Na.Alb $=$ H. Alb $+\mathrm{NaHCO}_{3}$. BZ XV II4.

Spring I896 Deltas formed by precipitation of mud by salts of sea water. NR.

Stadler \& Kleemann I9I I Adsorption of $\mathrm{NH}$ by erythrocytes. BZ xxxvi $30 \mathrm{r}, 32 \mathrm{r}$.

Starling I899 Diffusion from tissues into blood. Osmotic pressure of proteins, JP xxiv 3I\%.

I9I5 "Principles of Human Physiology." Phila.

Stempell I9I4 Contractile vacuole. ZJ xxxiv 437.

Stern I9I2 Osmotic pressure of concentrated solution. ZPC 1xxxi 44r.

Stewart I897 Electric conductivity of erythrocytes. ZP xi 332.

1899 Increase in conductivity of erythrocytes. Method of determining their volume. JP xxiv 2II, 356.

I90I In hemolysis salts come out before hemoglobin. JP xxvi 470. Ig09 Hemolysis. JPET i 49.

Stieglitz 1908 Catalysis. ACJ xxxix 62.

Stoklasa 1908 Ash of azobacter $=\mathrm{K}_{2} \mathrm{HPO}_{4}$, ZBk xxi. I9I6 Significance of $\mathrm{K}$ in plants. BZ lxxiii I0\%.

Strachan \& Chu I9I4 Transference no. of I. JACS xxxvi 810.

Straub I904 Adsorption of veratrin by heart of aplysia. AF i 65.

Stratub I9I2 Salts produce current of injury of heart. ZB lviii. I9I3 Effect of alkaloids on muscle and heart. ZP xxvi 990.

Stübel I9I I Fluorescence of tissues in ultraviolet light. AGP cxlii I. I9I4 Ultramicroscopy of blood coagtrlation. AGP clvi 361 .

Sumner I905 Osmose and fish. BBF Xxv 53. rgo7 Osmose and fish. AJP xix 61.

I9I I Objective study of sight in fishes. JEZ $\times 409$.

Svedberg Igog Colloidal sulphur. ZPkC 1xvii 249.

I909 No boundary between solutions and sols. ZPkC lxvii 105-25r.

I9I0 Brownian movement. KZ i.

Swellengrebel I905 Plasmolysis of bacteria. ZB xiv 374.

Szili Ig06 PH of fetal blood. Dogs killed by infusion of $\mathrm{HCl}$ at $\mathrm{PH}$ $=6.05$. AGP $\mathrm{cxv} 72,82$.

\& Friedenthal 1903 Buffers for Ringer's fluid. AAP 550.

Szuics 1910 Permeability. SWA cxix 737. IgII Effect of electric current on permeability. JWB lii 326. 
I9I2 Al ". increases permeability of spirogyra. JWB lii 85.

I9I3 $\mathrm{Al}^{\cdots}$ increases viscosity of spirogyra $\mathrm{H}_{2} \mathrm{O}_{2}$ kills. JWB lii 269.

Szyszkowski 1908 Adsorption on foam. ZPkC lxiv 387.

Tamman I888 Isosmotic solutions. APk xxxiv.

I892 Permeability of Traube's membranes. ZPkC X 255.

Tanaka I9I I Acids on castor bean lipase. JCET v 25.

I9I2 PH and lipase. JCET v I25.

Tangl Igo6 PH of stomach. AGP cxv 64.

\& Farkas I904 Respiration of fish eggs. AGP civ 624.

Tashiro I9I3 $\mathrm{CO}_{2}$ production by nerve. AJP xxxii I07, I37.

\& Adams IgI4 Anesthesia and $\mathrm{CO}_{2}$ by nerve. JZP-CB i 450.

Taylor 1906 Synthesis of fat by lipase. JBC ii 87 .

I907 Synthesis of protein by trypsin. JBC iii 87.

I9I5 "The Chemistry of Colloids." Longmans.

Teague \& Buxton I906 Charge of yeast reversed by ions. ZPkC 1vii 76. I907 Immune bodies cathodic. JEM ix 254.

1907 Dispersion of colloids. ZPkC $1 \times 479$.

Temple I9r5 Salting out of alcohol. Thesis. U. of Minn.

Tennent I9Io PH and Mendelian dominance. CIWP (No. 132) 117.

Thiel \& Strohecker Igr4 Hydration of $\mathrm{CO}_{2}$ and dissociation of $\mathrm{H}_{2} \mathrm{CO}_{3}$. BDCG xlvii 945 .

Thiselton-Dyer I899 Seeds live at $-250^{\circ}$. PRS 1xv 36r.

Thomas I9I5 Anesthetics increase viscosity of lecithin suspensions. JBC xxiii 359.

Thornton rgro Naturally cathodic plant cells, PRS (B) 1xxxii 638 .

Thunberg I9I I Autoxydation of lipoids. SAP xxiv 90.

Tigerstedt Igro Handbuch der Physiologischen Methodik. Leipsic.

Titoff I9Io Adsorption and solid solution. $\mathrm{ZPkC}$ 1xxiv $64 \mathrm{I}$.

Toda \& Taguchi I9I3 $\Delta$ of frog's urine. ZPC 1xxxvii 37I.

Torrey I9I2 $\mathrm{O}_{2}$ and polarity. U. Cal. Pub. (Z) ix 249.

Tower $1896 \mathrm{KCl}$ lessens diffusion potential. ZPkC.

Traube, I. Effect of ions on density of solution. ZAC iii 1 I.

I904 Stalagmometer. Surface tension of stomach less than blood. AGP cv 54I, 559.

1908 a Anesthetics inhibit hemolysis. Hemoglobin is hemolytic. BZ Ig08b Surf. Ten. norm, urine greater than blood. Less in nephritis. AGP cxxiii 4 I9.

I908c Surface tension and hemolysis. BZ xvi $37 \mathrm{I}$.

I909 Parthenogenesis. BZ xvi I82.

I9Io Haftdruck theory. BZ xxiv 323.

IgI I Haftdruck theory. AGP cxl Iog.

IgI2 Viscostagometer. BZ xlii 500 .

I9I3a Adsorption and solution. AGP cliii 273.

I9I3b Catalysis. AGP cliii 309.

I9I3C Many lipoid-insoluble dyes penetrate cells. BZ liv 305.

I9I5 Narcosis. AGP clx 50I.

\& Köhler I9I5 Solutions and swelling opposed to gelation and shrinking. JZPS ii 427.

\& Marusowa I9I5 Swelling of seeds. JZPB ii 370.

Traube, M. I867 Precipitation membranes. AAP 87, 609.

Trauty I905 Chemiluminescencz. ZPkC liii I.

Trendelenburg I9I2 Effect of salts on smooth muscle. AEP 1xix 79.

Trillat I904 Oxidase action of Mn + colloid. CRA cxxxviii 274.

Tröndle I910 Light increases the permeability of plant cells. JWB xlviii 171.

True IgI4 Toxicity of Aq. dist. AJB i 255.

1915 Conversion table for sea water. $S$ xlii 732. 
Tschagowetz 1907 Bioelectric currents. ZB 1247.

Turner I9I5 "Molecular Association." Longmans.

Tyndall r869 Tyndall phenomenon. PM (4) xxxvii 384.

Tyrode I910 Saline purgatives. Perfusion fluid. AIPd xx 20 S.

Underhill I916 Acidosis causes sugar retention. PSEB xiii iाt.

Urano I908a Accommodation of nerves to anisotonic solutions. ZB xxxii 459 .

I908b $\Delta$ of frog's muscle press juice $=.62^{\circ}$. ZB (NF) xxxii 212.

I908c Permeability of muscle. ZB (NF) xxxiii 483 .

Usher \& Priestly 1906-II Photosynthesis. PRS (B) 1xxvii 369; 1xxx 318; lxxxiv ror.

Usui I9I2 Part. coef, erythrocytes same after extracting lipoids. ZPC 1xxxi I73.

Van Bemmeln 1899 Spontaneous alteration of vapor pressure of gels. ZAC $\mathrm{xx}$.

Ig00 $\mathrm{K}^{\circ}$ of $\mathrm{K}_{2} \mathrm{SO}_{4}$ adsorbed leaving $\mathrm{H}_{2} \mathrm{SO}_{4}$ in solution. $\mathrm{ZAP}$ xxiii III, 32I.

Van Calcar \& Loby de Bruyn Igo4 Precipitation of dissolved crystalloids by centrifugal force. RTCP xxiii 218 .

Van der Laan I9I5 $\Delta$ of blood same as milk. BZ lxxi 289 .

Van Slyke \& Cullen IgI4 Formula for urease action. JBC xix I4I.

-, Cullen \& Stillman I9I5 $\mathrm{CO}_{2}$ in blood increased after meals. PZEB xii 184 .

\& Zacharias $19 \mathrm{r} 4 \mathrm{PH}=8.7$ for urease-urea combination. $\mathrm{PH}=7$ for urease decomposition. JBC xix I8I.

Van Rysselberghe I899 Turgor regulation. Plant cells permeable to $\mathrm{KNO}_{3}$. MARB 1viii I.

I90I Temperature coefficient of rate of plasmolysis. BARB I73.

Van't Hoff I887 Formula for osmotic pressure. ZPkC i 488.

Vernon 1904 Antitrypsin. JP xxxi 346.

IgIr Indophenol oxidase. JP xlii 402.

I9I2a Anesthetics below a certain high concentration do not affect indophenol oxidase. JP xlv 197.

19rab Toxicity of anesthetics to oxidase, BZ xlvii 375 .

rgr3 Autocatalysis of trypsinogen. JP xlvii 325 .

I9I4 Oxidation and lipoids. $\mathrm{BZ} 1 \mathrm{x} 202$.

Verworn 1909 Theory of narcosis. DMW (No. 37).

I9I3 Irritability. New Haven. Yale Univ. Press.

Verzar igraa Perfused muscle continues high rate of respiration minutes after tetanus. JP xliv 243.

I9I2b Thermo-current of nerve. AGP cxliii 252.

I9I2C Lack of oxygen and tissue respiration. JP xlv 39.

Voigt I9I4 Kupfer cells of liver take up colloidal silver. DMW (No, IO).

Von Brücke 1908 Non contracting muscle may produce action current. AGP cxxiv 215 .

Von Dungern \& Coca 1907 Hemolysis. MMW (No. 47). I908a Hemolysis. BKW 7.

I908b Hemolysis. MMW xlv 105.

1908c Hemolysis. BZ xii 407.

Von Elissafoff I9I2 Effect of ions on electroendosmose. ZPkC 1xxix 385.

\& Freundlich IgI2 Electric polarization of glass. ZPkC 1xix 385 .

Von Erlanger I897 Surface movements of nematode eggs. BC xvii I52, 339.

Von Fürth \& Lenk IgI I Muscle rigor. BZ xxxiii 34I.

Von Halban Ig09 Temperature coefficient of chemical reactions. ZPkC 1xvii 169 .

Von Jaksch 1890 Effect of diet on gast. acidity in children. ZKM xxiv 565. 
Von Schroeder I903 Viscosity of gelatine increased by acids and alkalis. $\mathrm{ZPkC}$ xlv 88.

Von Veimarn Igro Colloid state of crystalloids. Submicrons in gelatine. $\mathrm{KZ}$ vi 277 , vii.

Von Wyss Ig08 Reduction of $\mathrm{Cl}$ in blood by feeding $\mathrm{NaBr}$ and its effect on nervous system. AEP lix 186.

Von Zeynek \& Von Bernd igIo Stimulation impossible with 100,000 alterations per sec. AGP cxxxvii I.

Wachendorff IgI I Respiration of infusion. ZAP xiii Ior.

Wächter I905 Permeability to sugar. JWB xli I65.

Waentig \& Steche PH for catalase. ZPC Ixxii 226, 1xxvi 177.

I9I2 Action of catalase on $\mathrm{H}_{2} \mathrm{O}_{2}$ is adsorption catalysis. ZPC $1 \times x i x 446$.

Wagner 1890 Lyotrope series and viscosity. ZPkC v $3 \mathbf{I}$.

Wagner, H. I9I4 Action of light on chlorophyll. PRS (B) 1xxxvii 386.

Walbum Igr 3 Protein bleaches many indicators. BZ xlviii $291 ; 1346$.

Walden I892 Permeability of Traube's membranes, ZPkC x 699.

Walker, J. Igro "Introduction to Physical Chemistry." 6th Ed. Macmillan.

\& Cormack I900 Dissociation constants of weak acids. JCS 1xxvii I3.
\& Marshall IgI3 Hemoglobin positive. JACS xxxv 820.

Waller Igoo Electric effect of light on leaves. JP xxv (p. xvii).

Ig04 Increased conductivity of plant on stimulation. JLS (Bot.) xxxvii 32.

Wallace \& Cushing 1898 Rate of diffusion and of absorption by gut. AJP i 4 II.

Walpole I9r4 $\mathrm{H}_{2}$ electrode. BJ viii I3I. I9I4 Aggregation of sols. BJ vili I7o.

Walters I9I2 Reaction products on trypsin. JBC xii 43.

Warburg, E. I899 Formula for alternating current. APk 1xvii 497.

Warburg, O. Igo8 Oxidation of egg increases on fertilization. ZPC lvii I. I909a Oxidation of erythrocytes. ZPC lix II2.

Igogb Cleavage of egg may be prevented by $\mathrm{OH}^{\prime}$ which increases oxidation. ZPC 1x 443.

Igogc $\mathrm{CO}_{2}$ analysis. $\mathrm{ZPC}$ lxi $26 \mathrm{I}$.

I9roa Oxidation in egg not dependent on cleavage. ZPC lxvi 305.

Iglob Oxidation and anesthetics. ZPC lxix 452.

I9r1a Cell respiration and structure. MMW lviii 289.

I9I Ib Oxidation of cells. ZPC $1 \times x$ 4I3.

I9IIC Oxidation and anesthetics. ZPC Ixxi 479.

I912a No respiration in ground erythrocytes. AGP cxlv 277.

Igrab Fermentation and oxidation. MMW (No. 47).

I9I2c HCN and oxidation. ZPC Ixxvi 331 .

I9I3 Respiration of liver granules. AGP cliv 599.

I9I4 Blood charcoal oxidizes oxalic acid. AGP $\mathrm{clv} 547$.

I9I5 Egg respiration. AGP clx 324.

- \& Meyerhof I912 Cell respiration and structure. AGP cxlviii 295. I9I4 Oxidation of lecithin by iron. ZPC lxxxv 4I2.

- \& Wiesel I9I2 Action of anesthetics on anaerobic processes and enzymes. AGP cxliv 465.

Warner I9I4 Formaldehyde from chlorophyll. PRS (B) 1xxvii 378.

Washburn Igog Lyotrope series and $\Delta$. JACS xxxi 322.

IgI5 "Principles of Physical Chemistry." N. Y. McGraw-Hill.

- \& Bell I9I3 Apparatus for elec. conductivity. JACS xxxv 177.

Wasteneys 1916 Oxidation in egg reduced by stopping development. JBC xxiv $28 \mathrm{I}$.

Webster I902 Absorption of liquids by tissues. Decennial Pub. Univ. of Chicago $x$ 105.

Weinland 1894 Effect of salts on cilia. AGP lviii I05. 
Weizsäcker I9I2 Heart beat in KCN. AGP cxlvii I35.

Wessely 1903 Absorption of subcutaneous injections. AEP xlix 412.

Whatmough I902 Surface tension measurement. ZPkC Xxxix I26.

White IgIr Viscosimeter. BZ xxxvii 48r.

Whitney I908 Dessication of rotifers. AN xlii 665 .

- \& Blake I904 Polyvalent ions and cataphoresis. Removal of $\mathrm{Cl}$ from albumin. Peptisation. JACS xxvi I339.

\& Ober I902 Ion equivalents for precipitating sols, $\mathrm{ZPkC}$ xxxix 630.

Widmark IgIo $\Delta$ of fresh water animals. ZAP $x$ 43I.

Igr I Thumberg-Winterstein micro-respirometer. SAP xxiv 321.

Wiedemann 1852 Cataphoresis and electroendosmose. APk lxxxvii 321.

W \& Luedeking 1885 Heat of swelling of colloids. APk xxv I45.

Wijs 1893 Dissociation of water. ZPkC xii $5 \mathrm{I} 4$.

Wilke \& Meyerhof IgIo Polarization and colloid precipitation with alternating current. AGP cxxxvii.

Williams, Riche \& Lusk Igrz Dynamic action of protein. JBC xii 349.

Wilsmore I90I Lyotrope series and electrolytic solution tension. ZPkC xxxvi 91 .

Windaus 1908 Reaction between saponin and cholesterin. BDCG xlii 238.

Windle I 895 Electric effects produced by blood circulating in magnetic field. JAP xxix 346 .

Winkleblech I90I Ampholytes, ZPkC xxxvi 546.

Winterstein I897 Death rigor of muscle does not occur in $\mathrm{O}_{2}$, AGP cxx 225.

I910 Handbuch der vergleichende Physiologie.

I9I2 Microrespirometer. $\mathrm{BZ}$ xlvi 440 .

I9I3 Anesthetics do not lessen solubility of $\mathrm{O}_{2}$ in oil. BZ li 143 .

I9I4 Narcosis with alcohol increases oxidation of spinal cord. BZ $1 \mathrm{xi} 8 \mathrm{I}$.

Woelfel I 908 Distribution of salts in hemolysis. BJ iii I46.

Wolff, F. 1908 Temp. coef, of Weston cell. BBS v 309.

Wolff, J. I908 Oxidase action of colloidal ferric-ferrocyanide. CRA cxlvii 745 .

Wood 1903-6 Affinities of feeble bases. TCS 1xxxiii 568, 1xxxix I839. I908 Amphoteric metallic hydroxides. TCS xciii 4II.

Woodland IgII Gas secretion by fish. AA x1 225 .

Worm-Müller 1870 Membranes and transference numbers. PAP cx1 II6.

Yasuda Ig00 Acclimatization. JCS xiii ror. I900 Turgor regulation. JCS xiii ror.

Yatsu I905 Cytasters in enucleated eggs. JEZ ii 287.

Ylupoe 19'3 Isoelectric point of casein. $Z$ KK viii 224 .

Zamidzki Igo3 Adsorption on foam. ZPkC xlii 6I2.

Zangger 1908 Membranes can act like valves. Alcohol increases permeability of yeast to enzymes (I44). EP vii 99.

Zsigmondy 1906 Colloidal gold. ZPkC 1vi 65. Ig06 Sols without Tyndall phenomenon. ZEC xii 63I.

1912 Submicrons in gelatine. $\mathrm{KZ} \times \mathrm{i} 145$.

I9I2 "Kolloidchemie" (p. 249). ז/2\% gelatine submicrons on cooling. I9I3 Ultramicroscope. $\mathrm{PZ}$ xiv 975.

\& Bachmann I9I4 Immersion ultramicroscope. KZ xiv 28ז.

Zuelzer Ioo7 Addition of sea water stops contractile vacuole. Sitzb. Ges. Natf. Freunde. 90. 


\section{INDEX TO LITERATURE LIST}

Gentral : Abderhalden, Asher and Spiro, Bayliss, Griffiths, Hamburger, Hammersten, L. Henderson, Höber, H. C. Jones, Jordan, Koeppe, Landolt-Börnstein, Lusk, McClendon, McKenzie, Matthews, Mellor, Nägeli, Nagel, Neuberg, Nernst, Oppenheimer, Wm. Ostwald, Ostwald-Luther, Philip, Pütter, Starling, Tigerstedt, Turner, Washburn, Walker, Winterstein.

Absolute Weight of Molecules: Perrin.

ABsorption: Durig, Fleisher and Loeb, Friedenthal, Hamburger, Höber, Muller, Reid, Webster, Wesley.

AcıDosis: Allen, Higgins and Means, Howland and Marriott, Masel, Novac, Leimdörfer and Porges, Packard, Peabody, Polanyi, Porges, P., Leimdörfer and Markovici, Poulton, Reicher, Rolly, Ryffel, Sellards, Szili, Underhill.

Adaptation: I_oeb and Wasteneys, Schneider, Urano, Yasuda.

ADSORPTION: Barger and Starling, Evans, Herzog and Betzel, Lewis, Liebreich, Michaelis and Ehrenreich, Milner, Morawitz, Wo. Ostwald, Paul, Birsetin and Reuss, Röentgen and Schneider, Rona and Michaelis, Stadler and Kleeman, Straub. Ad.-Compounds and Colloid Reactions: Iscovesco, McClendon, Noguchi, Windaus, Ransom. AD, of Coltords: Dawe, Hedin, Landsteiner and Uhlirz, Michaelis, M. and Rona, Porter, Quincke, Schmidt-Nielson. Ad. AND Electric Charge: Bayliss, PeletJolivet and Anderson. AD. ON FOAM: Benson, Szyszkowski, Zawidzki. Ad. And Surface Tension Formula: Duclaux, Freundlich, Kuster. Ad. Saturation: Marc, Schmidt. Ad. and Solid Solution: Davis, Marc, Titoff, Traube. Competitrve AD.: Biltz and Steiner, Freundlich and Losev, F. and Neumann, Harris, Jahnson-Blohm, Lachs and Michaelis, Michaelis and Rona, Rona and Tosh, Van Bemmeln.

AmeBoid Motion: Bernstein, Hirshfeld, McClendon, Montgomery, Rhumbler, Ross.

Alimentary Tract: Embden and Kraus, Pavloff.

ANestherrcs: Boothby, Carlson, Frey, Fühner, Hamburger and de Haan, Johannsen, Lee, L. and Salant, Meltzer and Auer, Meyer, Overton, Otto, Salzmann, Traube. Ar. and Cells: Gros, Lillie. AN. ANd ColLorDs: Calugareanu, Goldschmidt and Pribram, Koch and McLean, Lillie, Moore and Roaf, Pribram. AN. AND Oxidation: Alexander and Cserna, Burker, Mansfeld, Meyerhof, Nothmann-Zuckerkand1, Tashiro and Adams, Vernon, Verworn, Warburg. An. And Permeability: Höber, Joel, Lillie, McClendon, Meyer, Osterhout. Storage of AN.: Choquard, Mansfeld.

Antitoxic Action: Henderson, Ishikawa and Löwi, Lillie, Robertson. Artifictal Membranes: Zangger, Bartell, Bayliss, Bein, Bigelow and Bartell, W. Brown, Harvey, Lepeschkin, Meigs, Morse and Horn, Nathanson, Wm. Ostwald, Pascucci, Ruhland, Tamman, M. Traube, WormMuller.

Artificial Parthenogenesis: Bataillon, Delage, Glaser, Harvey, Lillie, Loeb, McClendon, Morse, Robertson, Traube.

Aurocatalysis: Herzog, Hoyler, Philoche, Tanaka, Vernon.

Autolysis: Benson and Wells, Bradley and Taylor, Chiari, Grode and Lesser, Lyon and Shackell, Morse.

Bioelectric Phenomena: Bernstein, Beutner, Boruttat, Brünings, 
D'Arsonval, Galeotti, Garten, Hyde, Mathews, Oker-Blom, Tschagowetz. B. P. of GLands: Cannon and Cattell. B, P. of Heart: Fleischhauer, Hirschfelder, Straub. B. P. of Muscle: Frolich and Meyer, Pauli and Matula, Piper, Van Brucke. B. P. of Plants: Burdon-Sanderson, Loeb and Beutner, Waller. B. P. of SkIN: Höber, Lesser, Schwartz.

Blood: Barcroft, Abderhalden, Böhm, Denis, Dreyer and Ray, Erb, Heller, Keith, Rowntree and Geraghty, McClendon, Magnus, Pascucci, Plesch, Scott. Coagulation of B.: Deetjen, Franz, Haycraft, Hekma, Howell, Pekelharing, Ringer and Gainsbury. B. GAses: Barcroft, Christiansen, Douglas and Haldane, Haldane, Hill, $H$. and Nabarro, Krogh and Krogh, McClendon, Murlin, Edelmann and Kramer, Nicloux, Van Slyke, Cullen and Stillman. Ionic Exchange with B. Corpuscles: Hamburger, H, and Van Lier, Koeppe, Spiro and Henderson.

Brownian Movement: Einstein, Perrin, Svedberg.

Buffers : Bugarszky and Liebermann, Clark and Lubs, Corral, Davidsohn, Friedenthal, L. Henderson, H. and Spiro, Hoffmann, Levy and Rowntree, Palitzsch, Prideaux, Szili and Friedenthal.

Calomel Electrone: Auerbach, Lipscomb and Hulett, Loomis and Acree, McClendon, Richards.

CALORIMETRY: Bohr and Hasselbach, Glaser, Hill and Wezsacker.

CARBon Droxide: Auerbach and Pick, Boothby and Peabody, Findlay, L. Henderson, H. and Black, Hooker, Johnston, Krogh, Lowy and Zuntz, McClendon, McCoy, Momose, Thiel and Strohecker, Warbutg. Alveolar $\mathrm{CO}_{2}$ : Begun, Hermann and Munzer, Higgins.

Catalyzers : Bredig, B. and Fajans, Dietz, Ernst, Faraday, Frankforter and Kritchensky, Heimrod, Kastle, Meyerhof, Nielsen, Schoebein, Stieglitz, Traube.

Cell Division: Baltzer, Bonnevie, Child, Conklin, Fischel, Gallardo, Gardiner, Lillie, Loeb, L. and Wasteneys, Lyon, McClendon, Mathews, Prenant, Richards, Robertson, Schimkewitsch, Von Erlanger, Warburg, Yatsu.

Cell Structure: Chambers, Conklin, Fischer, Gardener, Gerassimow, Goldschmidt and Poppoff, Hamburger, Hardy, Köhler, Warburg.

Centrifuges Banta and Gortner, Grijns, Hamburger, Hedin, Koppe, McClendon, Van Calcar and Loby de Bruyn.

Changes in Permeability: Garmus, Löwe, McClendon, Mayerhoffer and Stein, Stewart, Szücs. C. I. P. of EGGs: Gray, Lillie, Loeb, Lyon and Shackell, McClendon. C. I. P. of Muscle: Höber. C. I. P. of Nerve: Bernstein. C. I. P. of Plants: Fernbach, Flüri, Kisch, Lepeschkin, Osterhout, Pantanelli, Szücs, Trondle

Cilia: Höber, Lillie, Maxwell, Weinland.

Colsoins: Bechhold, Freundlich, Jordis, Krafft, Lottermoser and Rothe, Osborne, Wo. Ostwald, Pauli, Ruhland, Svedberg, Taylor, Zsigmondy. Coagulation of C.: Bovie, Buxton and Rahe, Buxton and Shaffer, Chick and Martin, Hardy and Whetham, Harlow and Stiles, Jorissen and Woudstra, Pascheles, Pauli, Quagliariello, Shaklee and Meltzer. Getation of C.: Heilbrunn, Walpole. Precipitation of C.: Batelli and Stern, Billiter, Freundlich, Freundlich and Schucht, Friedemann and Friedenthal, Hofmann, Landstein and Jagic, Mathews, Michaelis and Rona, Paine, Pauli, P. and Fletcher, Porges and Neubauer, Schulze, Whitney and Ober, Wilke and Meyerhof. Solation of C.: Hardy, Lottermoser. FreezrngOut of C.: Fischer and Jensen, Lottermoser. Protection-C.: Faraday, Schaffnit.

Concentration Cells: Abegg, Auerbach and Luther, Bates, Bennett and Cole, Beutner, Bjerrum, Bugarsky, Cremer, Cummings and Gilchrist, Cybulsky, C. and Dunin-Borokowski, Drucker, Haber and Klemensiewicz, Henderson, McInnes, Mines, Nernst, N. and Riesenfeld, Planck, Tower.

Conduction of Excitation: Bose, Carlson, Fitting, A. V. Hill, Keith 
and Flack, Lapique and Legendre, Lillie, Lodholz, Lucas, McClendon, Mayer.

Contractile VAcuole: Rhumbler, Stempeli, Zuelzer.

Cytolysis: Lillie.

Density: Lyon, Traube.

Desiccarion: Becquerel, Dreyer and Walker, Jacobs, Shattock and Dudgeon, Whitney.

Difrusion: Abel, Rowntree and Turner, Bechhold and Ziegler, Hamburger, Hedin, Höber, Michaelis and Rona, Rona and György, Scheffer, Wallace and Cushny. D. of Colloids: Flexner and Noguchi, Graham, Herzog and Kasarnowski, Mayerhofer and Pribram. Wood.

Dissoclation of AmpHolytes: Bredig, Kanitz, Lunden, Robertson,

Disinfection: Clark, Czapek, Dreser, Eisenberg and Okoloska, Kahlenberg, K. and Austin, K. and True, McClintic, Mathews, Morawitz, Norton and Hsu, Nothmann-Zuckerkandl, Paul, P. and Kronig, Pulst, Scheurlin, S. and Spiro, Spiro and Bruns, Stevens, True.

Dynamic Áction of Protein: Lusk, Williams, Riche and Lusk.

Electric Charge: of Ceils: Cernovodeanu and Henri, Henri, Höber, Teague and Buxton, Thornton. E. C. of Colloids: Billiter, Biltz, Bottazzi, Burton, Buxton, Shaffer and Teague, Coehn, Freundlich and von Elissafoff, Henri and Mayer, Iscovesco, Knoblauch, Lewis, Linder and Picton, Luther, Peckelharing and Ringer, Perrin, McClendon, Pauli, Von Elissafoff and Freundlich, Walker and Marshall, Whitney and Blake. E. C. of Protoplasm: Brown, McClendon.

Electric Conductivity: Bugarsky and Tangl, Harris and Gortner, Noyes, N. and Melcher, Cooper and Eastman, Oker-Blom, Washburn and Bell. E. C. of Cells: Brown, Fränckel, Gray, Höber, McClendon, Stewart. E. C. of Tissues: Du Bois-Reymond, Galeotti, Kodis, McClendon, Waller.

Electric Organ: Bauer, Bernstein and Von Tschermak, Dahlgren, Garten.

Electric Polarization of Membranes: Bancels, Bayliss, Bethe and Toropoff, Beutner.

Electroennosmose: Bethe, Bournat, Byers and Walter, Von Elissapoff, Wiedemann, Windle.

Electrode Potential: Abl, Billiter, Nernst.

Electrolytes: Rona, R. and Michaelis, R. and Takahashi, Winkleblech.

Electrolytic Dissociation: Arrhenius, Bousfield, Denham, Ellis, His and Paul, Millikan, Noyes and Blanchard, Wm. Ostwald, Rudolphi, Scudder, Van't Hoff, Walker and Cormack, Wood.

El.ectrolytic Solution Tension: Bechhold, Haber, Heald, Lewis and Keyes, L. and Rupert, McGuigan, Mathews, Michaelis, Nernst.

Electrometers and Galvanometers: Burch, Einthoven.

Emulsions: Iscovesco, McClendon.

Emulsoids: Galeotti, Hatschek, Traube and Kohler, Van Bemmeln.

Enzymes: Euler, Bradley, Emmerling, Falk and Nelson, Frankel, Hudson, Koopman, Lyon, McClendon, Mayer, Meyer, Morse, Oppenheimer, Pierce, Porter, Schutz, Sedgwick and Schlutz, Slator, Van Slyke and Cullen, Warburg. E. AND AsYMMETRY: Abderhalden and Guggenheim, A. and Pringsheim, Bayliss, Dakin, Erlenmeyer, Bredig and Fiske, Fajans, Fischer, F. and Abderhalden, Herzog and Meier, Pasteur. E. InHIBIToRs: Abderhalden and Gigon, Armstrong, Bigelow, Burge, Cathcart, Hammill, Hedin, Michaelis and Menten, Pfeffer and Hansteen, Richards, Souza, Vernon, Walters. E. And Surface Phenomena: Beard and Cremer, Goldschmidt, Meyerhof, Waentig and Steche, Warburg and Wiezel. E. Synthesis: Armstrong, Bradley, A. C. Hill, Kastle and Loevenhart, Rosenthaler, Rubner, Taylor. 
Equilibrium Point: Knupfer and Bredig, Wm. Ostwald. E. P. of ENZYMES: Acree and Johnson, Bodenstein and Dietz, Euler.

Excitability: Joseph and Meltzer, Overton, Koltzoff, Schwarz.

EXCRETION: Asher and Waldstein, Bainbridge Collins and Menzies, Barcroft and Brodie, B. and Straub, Barlow, Beddard, Bock and Hoffmann, Burian, Carrel and Guthrie, Cushny, Ghiron, Höber and Konigsberg, Knowlton, Nishi, Pototsky, Ribbert, Ringer, Sollman.

Exudates: Chiari and Januschke, Januschke.

Fertilization: Bachmann and Runnstrom, Glaser, Jacoby, Lillie, Loeb, McClendon, M. Morse.

Fertilization Membrane: Harvey, Heilbrunn, Herbst, McClendon, Schucking.

Filtration: Bigelow, Heymans, Lottermoser.

Freezing Point: Burian and Drucker, D'Arsonval, Garrey, Gortner and Harris, Harris and Gortner, Loomis, MacCallum and Benson, Sabbatani, Toda and Taguchi, Urano, Widmark. F. P. of Blood: Botazzi, B. and Ducceschi, Buglia, Carlson, Greer and Luckhart, D'Errico, Dekhuisen, Fano and Botazzi, Franz, Höber, Von Koranyi, Kovacs, Nolf, Phillipson Hennevart and Thieren, Portier, Quinton, Rodier. F. P. of FISH: Greene, Loeb and Wasteneys, G. Scott, Sumner. F. P. of Blood of Mammals: Atkins, Salge, Schulz, Van der Laan. F. P. of Eggs: Backman, B. and Runnstrom, B, and Sundberg, Bialaszewicz. F. P. of Plants: Atkins, De Vries, Fitting, Gortner and Harris, Harris and Gortner.

Growth : Congdon, Davenport, Donaldson, Frozee, Gager, Harrison, Hyde and Sprier, Jackson.

Haptogen Membranes: Hofmann, Lewis, Metcalf, Pickering, Ramsden, Rohde, Schutt, Shorter.

HEART: Busquet and Pachon, A. J. Clark, Mines, Ringer.

Hemolysis: Stewart, Fühner and Neubaur, Girard, Gros, Guthrie, Hamburger, Handowsky, Hirschfeld, Höber, H. and Nast, Kyes, McPhedran, Michaelis and Takahashi, Miculicich, Neufeld and Handel, Noguchi, Port, Roaf, Rywosch, Traube, Von Dungern and Coca, Woelfel. HEREDITY: Gregor Mendel, Tennent.

Hydration: H. C. Jones, Lewis, Pauli and Falek.

Hydrogen Electrode: Clark, Hemptinne, Hoitsema, Lewis and Lacey, McClendon, Mond, Ramsay and Shields, Myers and Acree, Richardson Nicol and Parnell.

Hydrogen Ion Concentration: Emslander, Hasselbalch, Henze, Herbst, Howe and Hawk, Marriott, Michaelis, Okada, Schmidt, Sörensen, Walpole. PH of Bacterial Culture Media: W. M. Clark,. C. and Lubs. PH of Bloon: Agazzotti, Benedikt, Bottazzi and Craifaleanu, Farkas, F. and Scipiades, Friedenthal, Hasselbalch and Lundsgaard, Henderson, Höber, Konikoff, Kreibich, W. Loeb and Higuchi, Lundsgaard, Mathison, McClendon, Menten and Crile, Milroy, Mines, Pfaundler, Quagliariello, Rowntree, Marriott and Levy, Salge. PH AND Enzymes: Allemand, Auerbach and Pick, Compton, Davidsohn, Fernbach, Hudson, Huenekens, Kanitz, Palitzsch and Walbum, Ringer, R. and Van Trigt, Sörensen, Tanaka, Van Slyke and Zacharias, Wäntig and Steche. PH of URINE: Henderson, Höber and Jankowsky, Rossi.

Hydrolytic Dissociation: Shields.

Hysteresis: Danysz, Freundlich.

ImMune Bodres: Biltz, Bordet, Beniasch, Bernstein and Simonds, Craw, Eisenberg and Volk, Field and Teague, Porges, Teague and Buxton.

Ions, Áction of: Beneche, Eisler, R. Ellis, Forster, Frey, Gray, Grünwald, Guthrie and Ryan, Herbst, Loeb, Mathews and Brooks, Mines, Morgan and Stockard, Osterhout, Ringer and Phear, Spaeth, Von Wyss. Antagonistic Action of I.: Bernoulli, Fischer, Freund, Galeotti, Linder 
and Picton, Loeb, L. and Cattell, L. and Gies, Loew, Meltzer and Auer, Osterhout, Spaeth.

IONIC SPEED: Bredig, Kohlrausch.

Ion-Protein Compounds: Cohnheim, Handowsky, Manabe and Matula, Pauli and Handowsky, Pfeiffer and Von Modelsky, Rohonyi, Rona and György, R. and Takahashi, Simon.

InDICATORs: Fowler, Bergeim and Hawk, Friedenthal, Harvey, Levy, Rowntree and Marriott, Lubs and Clark, McClendon, Michaelis and Davidsohn, Wo. Ostwald, Palitzsch, Salm, Scatchard and Bagert, Sörensen and Palitzsch, Walbum.

InHIBITION: Chiari and Frolich, Gaskell, Hagglund, Hagan and Ormond, Hering, Howell and Duke, Meek and Eyster.

INTESTINES: Cohnheim, Hooker, Quagliariello, Rona and Neukirk, Tyrode.

Isoelectric Point: Buglia, Hardy, Kozawa, Landsteiner, Michaelis, $M$. and Davidsohn, M. and Rona, Schorr, Ylupoe.

Light Perception: Eldridge-Green, Hartridge, Henri.

LYMPH: Heidenhain, Leathes.

Lyotropic Series: Bechhold, Gouy, Grützner, Höber, H. and Waldenberg, Hofmeister, Kölichen, Loeb and Wasteneys, McIntosh, Pauli, Schade, Washburn, Wilsmore.

Mass Action LaW: Guldberg and Waage.

Micro Analysis: Hamburger, A. B. Macallum, McDonald.

MiLK: Abderhalden, Burri and Nussbaumer, Davidsohn, Friedenthal.

Muscle, Smooth : Fienga, Trendelenberg, Meigs, M. and Ryan, Pugliese. Striated M.: Höber and Spaeth, Mines, Beccari, Jensen and Fischer, Katz, Meigs, Von Fürth and Lenk, Winterstein. M. ConrracTION: Benedict and Cathcart, Berg, Bernstein, Cohnheim and von Uexkull, Cooke, Du Bois-Reymond, Ebbecke, Embden and Kondo, Engelmann, Fletcher, F. and Brown, A. V. Hill, Höber, Macallum, McClendon, McDougall, Meigs, Overton, Pauli, Ringer, Roth, Schwarz, Straub.

Negatrve OSmose: Bartell, B. and Hocker, Cohnheim, Dutrochet, Flusin, Freundlich, Girard, Graham, Hamburger, Heidenhain, Reid.

NEPHELOMETER: Richards, $R$. and Wells.

Nerves: Alcock and Lynch, Baeyer, Grützner, Lodholz, Lucas, Mathews.

Non-Electrolytes:' Gudzent, Loeb, Moruzzi, Temple.

Optical Rotation: Pauli, Samec and Strauss.

Osmosis: Aubert, Bartell.

Osmotic Pressure: Findlay, Arrenhius, Barger, Berkley and Hartley, Clowes, Friedenthal, Hedin, G. N. Lewis, Loeb and Wasteneys, H. N. Morse, Pfeffer, Ramsay, Raoult, G. G. Scott, Stern, Stumner, Tamman, Van't Hoff. O. OF Collords: Bayliss, Biltz, Donnan, D. and Harris, Ducceschi, Duclaux, Gatin-Gruzewska, Lillie, Moore and Roaf, Reid, Roaf, Starling. O. Regtration: W. J. Dakin, Fredericq, Garrey, Henri and Lalou, Mayenburg, Mouton, Overton, Pantanelli, Quinton, Schmidt-Nielsen, G. G. Scott, Sumner, Van Rysselberghe, Yasuda.

OxyHemoglobin: Barcroft and Camis, B. and Hill, Bohr, Donders, Douglas, Freundlich and Bjercke, Haldane and H., A. V. Hill, Hüifner, H. and Gansser, Wo. Ostwald, Scott.

Oxidation: Ehrlich, Banta, Brodie and Vogt, Drury, Fenton, Hyman, Krogh, McClendon, Mathews, Murlin and Kramer, Onaka, F. H. Scott, Thunberg, Warburg, Widmark, Winterstein, Wolff. O. AND ANESTHETICs: Batelli and Stern, Vernon, Warburg, Winterstein. O. IN CELLS: Batelli and Stern, Child, Harden and McLean, Heilbrunn, Höber, Itami and Pratt, Lillie, Loeb and Wasteneys, McClendon and Mitchell, Meyerhof, Moldovan and Weinfürter, Morawitz, Schlapper, Tangl and Farkas, Tashiro, Wachendorff, Warburg, W. and Meyerhof, Wasteneys. O. ENzymes: Bunzell, H. D. Dakin, Kastle, Bach and Chodat, Engler and Wild, 
Herzog and Meier, Moore and Whitley, Reed, Trillat, Vernon, Warburg, W. and Meyerhof. O. AND Oxygen Pressure: Bert, Demoor, Fallois, Henze, Lehmann, Loeb and Wasteneys, Mitscherlich, Pütter, Roaf, Torrey, Verzar. O. AND PH: Mathews, McClendon and Mitchell, Warburg. O. IN Tissues: Batelli and Stern, A. V. Hill, Verzar. O. IN CRUshed Cells: Batelli and Stern, Büchner and Rapp, Bunzel, Fletcher and Hopkins, Meyerhof, Warburg.

Partition Coefficient: Berthelot and Jungfleisch, Löwy, H. Meyer, Overton, Pohl, Reinders, Usui.

Permeability: Bartell, Bayliss, Bechhold, Bethe, De Vries, Endler, Harvey, Höber, Katzenellenbogen, Kite, Kuhne, L'Hermite, Loeb, Magnus, Sorgdräger and von Leeuwen, Mathews and Longfellow, Nernst, Overton, Riddle and Spohr, Rost, Schüking, Szücs, Walden. P. OF BLood Vessels: Magnus, F. H. Scott. P. to Dyes: Eisler and von Portheim, Bethe, Evans and Schulemann, Höber and Kempner, Kuster, Löwe, Michaelis and Davidsohn, Schulemann, Traube. P. OF EGGS: Brown, Harvey, Loeb, McClendon, Overton, Sollman. P. OF ERYThrocytes: Calugareanu and Henri, Constantino, Hamburger, Hedin, Höber, Kozawa, Masing, Moore and Roaf, Rona and Doblin, Rona and Takahashi, F. H. Scott, Stewart. P. of Glands: Asher and Karaulow, Siebeck. P. of Muscte: Fahr, Jacoby and Golowinski, Meigs, Overton, Siebeck, Urano. P. of Plants: Fischer, Harvey, McClendon, Merril, Osterhout, Overton, Paine, Wächter. P. of SEeds: Atkins, A. Brown, Shulh

PERIodictry: Bohn, Bredig, B. and Weinmayr, F. Darwin, Küster, Liesergang, Lyon.

Phagocytosis: Hamburger, $H_{\text {. }}$ and de Haan, H. and Hekma, Koltzoff, L. Loeb, Prowazek, Rhumbler, Savchenko and Aristovsky, Voigt.

Potentiometer: Bovie, McClendon.

Photogenesis: Callaud and Pelletier, Coblentz, Dubois, Harvey, Radziszewski, Stubel, Trautz.

PHotosynthesis: Allen, Biltz, Berthelot and Gaudechon, Bodenstein, Bunsen and Roscoe, Gibson and Titherly, Hallwachs, Hughes, Luther and Forbes, B. Moore, M. and Webster, Schryver, Sheppard, Usher and Priestley, Wagner, Warner.

Phystological or Perfusion Futids and Cell Media: Göthlin, Herlitzka, Lewis, Locke, Raulin. PH of P.: Mines, Rona and Wilenko, Schwartz and Lemberger, Schwyzer.

Plant Movements: Bose, Pringsheim, Ewart.

Plasma Membrane: Kuster, Lepeschkin, Prowazek, Quincke.

Plasmolysis: Bartetzko, Hamburger, Osterhout, Overton, Schwellengrebel.

Psychogalvanic Reflex: Gildemeister, Salamonson.

Refraction: Pearl and Gowan, Botazzi.

Regeneration: Cary, McCallum, Moore.

Respiratory Center: Campbell, Douglass, Haldane and Hobson, D., $\mathrm{H}$., Henderson and Schneider, Gasser and Loevenhart, Haldane and Priestley, Hasselbalch, Milroy, Porges, Winterstein.

Resistance: Hutchison, Wo. Ostwald, Pictet, Thistleton-Dyer.

SEA WATER: Allen and Nelson, Bethe, Clarke, Dittmar, Dole, Drew,

Forschhammer, Krogh, Macallum, Matthews, Palitzsch, Raben, True.

SECRETION : Bainbridge, Benrath and Sacs, Bergeim, Bohr, Botazzi and Enriquez, Fitzgerald, Mendel and Thatcher, Woodland.

Solubility: Bechhold and Ziegler, Bodlander and Fitting, Gudzent, Hulett, Wm. Ostwald, Pauli and Samec, Philip, Schade, S. and Boden, Vernon, Winterstein.

Stimulation: Adrian, Bethe, Chapin, Erlanger and Garrey, A. V. Hill, Höber, Lapique, Lillie, Loeb and Ewald, McClendon, Mathews, Nernst, N. and Barratt, Von Zeynek and von Bernd Verworm, Warburg. 
Stomach: Cannon, Carlson Orr and Brinkman, Davidsohn, Hess, Rehfuss, R., Bergeim and Hawk. PH of S. Contents: Boldyreff, Frankel, McClendon, Menten, Michaelis and Davidsohn, Rehfuss and Hawk, Tang1, Von Jaksch.

Surface Tension: Allen, Berczeller and Csaki, Billird and Bruyant, B. and Dieulafe, Botazzi, Colton, Cramer, Davis, Donnan and D., Duclaux, Dupré, Fanno and Mayer, Frenkel and Cluzet, Gibbs, W. J. Jones, Harkins and Humphrey, Kaufler, Kisch, W. "Lewis, Macallum, McClendon, Michaelis, Morgan, Quincke, Rayleigh Traube Whatmough. S. T. ớ Colloids: Berczeller, Botazzi.

SusPensions: Einstein, Freundlich and Mäkelt, Perrin, Spring.

Suspensotds: Barus, Bredig, Duclaux, Svedberg, Zsigsmondy.

SpermatozoA: De Meyer, Hirokawa, Koltzoff, F. Lillie.

Sweluing: Fischer, Demoor, Goodridge and Gies, Loeb, Meigs, Meyer and Cohn, Nasse, Schäper, Schloss, Traube and Marusawa. S. OF CoLLoIDS: Beutner, Chiari, Ehrenberg, Fischer and Moore, F. and Sykes, Hauberisser and Schönfeld, Henderson, Palmer and Newburgh, Lewites, Ludwig, Pauli and Rona, Posnjak, Samec, Schade, Spiro, Wiedermann and Ludeking. Osmotic S.: Beutner, Bialaszewicz, Hirokawa, McClendon. TURGor: Correns, Massart.

Synthesis: Kölichen, Loew. (See Enzymes.)

Temperature Coefricient: Bernstein, Clausen, Ganter, Kanitz, Krogh, Lucas, Masing, A. G. Mayer, A. Moore, Snyder, Van Rysselberghe, Von Halban.

Thermodynamics: Bernstein, Dupré, Gibbs, Lewis and Randall, Meyerhof, Verzar.

Tropisms: Jennings, Loeb. Galvano T.: Bancroft, Cohn and Barrat, Dale, Gassner, Greely, Loeb and Budgett, Ludloff, Mendelssohn. Geo T.: Czapek, Haberlandt, Kanda, Lyon, McClendon, Nemec. Pното T.: Ewald, Engelmann, Loeb, L. and Wasteneys, Mast. Rheo. T. : Allee, A. and Tashiro, Allen, Lyon.

Tyndall Phenomenon: Picton and Linder, Rayleigh, Tyndall, Zsigmondy.

Ultrafiltration: Bechhold, Burian, Hatschek, Ruhland.

Ultramicroscopy: Bachmann, Cesana, 'Cohn, Donnan and Potts, Gatin-Gruzewska and Biltz, Höber, Le Blanc, Linder and Picton, Menz, Michaelis and Pincussohn, M. and Rona, Perrin, Price, Rählmann, Siedentopf and Zsigmondy, Stübel, Teague and Buxton, Von Veimarn, Zsigmondy, Z. and Bachmann.

UNDERCOOLING: Bachmetjew, Kodis, Mitler-Thurgau.

VAsomotor Center: Mathison, Sollman and Pitcher.

Viscosity: Arrenhius, Wo. Ostwald, Szücs, Traube, Wagner, White. V. OF Blood: Albutt, Austrian, Bayliss, Boveri, Burton-Opitz, Heubner, McCaskey. V. OF ColnoIDs: Bingham and Durham, Hatschek, Laquer and Sackur, Michaelis and Mostynski, Pauli and Samec, P. and Wagner, Thomas, Traube, Von Schröder.

WATER: Armstrong, Bousfield and Lowry, Guye, Hulett, Kohlrausch and Heydweiller, Krogh, Lowe, Lowenherz, Lorenz and Boehi, Roaf, Sörensen and Palitzsch, Wijs.

Weston Celt: Rosa, Wolff. 
3

1 $-$ 

(3) (19) 1) (1.) 